\title{
Dynamic panel data models : theory and macroeconomic applications
}

Citation for published version (APA):

Hoogstrate, A. J. (1998). Dynamic panel data models : theory and macroeconomic applications. [Doctoral Thesis, Maastricht University]. Universiteit Maastricht. https://doi.org/10.26481/dis.19980129ah

Document status and date:

Published: 01/01/1998

DOI:

10.26481/dis.19980129ah

Document Version:

Publisher's PDF, also known as Version of record

\section{Please check the document version of this publication:}

- A submitted manuscript is the version of the article upon submission and before peer-review. There can be important differences between the submitted version and the official published version of record.

People interested in the research are advised to contact the author for the final version of the publication, or visit the DOI to the publisher's website.

- The final author version and the galley proof are versions of the publication after peer review.

- The final published version features the final layout of the paper including the volume, issue and page numbers.

Link to publication

\footnotetext{
General rights rights.

- You may freely distribute the URL identifying the publication in the public portal. please follow below link for the End User Agreement:

www.umlib.nl/taverne-license

Take down policy

If you believe that this document breaches copyright please contact us at:

repository@maastrichtuniversity.nl

providing details and we will investigate your claim.
}

Copyright and moral rights for the publications made accessible in the public portal are retained by the authors and/or other copyright owners and it is a condition of accessing publications that users recognise and abide by the legal requirements associated with these

- Users may download and print one copy of any publication from the public portal for the purpose of private study or research.

- You may not further distribute the material or use it for any profit-making activity or commercial gain

If the publication is distributed under the terms of Article $25 \mathrm{fa}$ of the Dutch Copyright Act, indicated by the "Taverne" license above, 


\title{
Dynamic Panel Data Models: \\ Theory and Macroeconomic Applications
}

\author{
Proefschrift
}

Ter verkrijging van de graad van doctor aan de Universiteit Maastricht, op gezag van de Rector Magnificus, Prof. dr. A.C. Nieuwenhuijzen Kruseman, volgens het besluit van het College van Decanen, in het openbaar te verdedigen op donderdag 29 januari 1998 om 16.00 uur

$$
\text { door }
$$

Adriaan Jan Hoogstrate 


\section{Promotores:}

Prof. dr. F.C. Palm

Prof. dr. G.A. Pfamn

Beoordelingscommissie:

Prof. dr. C.J.M. Kool (voorzitter)

Prof. dr. H.J. Bierens (Pennsylvania State University, USA

Katholieke Universiteit Brabant, Tilburg)

Dr. S. Schim van der Loeff

ISBN 90-5681-024-3 


\section{Preface}

The research presented in this thesis was funded by the Economics Research Foundation, which is part of the Netherlands Organization for Scientific Research (NWO). Part of the research was done while visiting the Southern Methodist University in Dallas. Chapter three is based on work with Franz Palm and Gerard Pfann. Chapter five is based on research in cooperation with Thomas Osang from the Southern Methodist University, Dallas.

\section{Acknowledgments}

Writing a thesis is a dynamic process in which a lot of factors determine whether the process will stay within acceptable boundaries and finally lead to a product which satisfies everyone concerned. I would like to thank all the people who helped me to keep this process within its boundaries.

I would like to start with my two supervisors Franz Palm and Gerard Pfann who initiated and supervised the whole process. Further I would like to thank Denis de Crombrugghe and Jean-Pierre Urbain for their helpful discussions.

In particular I would like to thank Herman Bierens who not only made it possible for me to visit the Southern Methodist University in Dallas, but who also helped me to get the process really in control. I also would like to express my gratitude to the people of the Economics Department of the Southern Methodist University for their hospitality during my visit: Thomas, Hideo, Kamal and Rupie, Donna and Ron thanks!

Friends are those people whom one likes to thank for more reasons than one can ever write down: Horst, Thomas and Bettina Danke!

Finally I would like to thank Lia for her support, encouragement, patience and love.

André Hoogstrate

November 1997. 


\section{Contents}

1 Introduction 1

1.1 Introduction . . . . . . . . . . . . . . . 1

1.2 Dynamic panel data models . . . . . . . . . . . . . . 2

1.3 Outline . . . . . . . . . . . . . . . . . . . 3

2 Maximum Likelihood Estimation $\quad 7$

2.1 Introduction . . . . . . . . . . . . . . 7

2.2 Analysis of dynamic panel data $\ldots \ldots \ldots \ldots \ldots$

2.3 Likelihood analysis of $\mathrm{AR}(\mathrm{p}) \ldots \ldots \ldots \ldots \ldots \ldots$

2.3.1 Derivation of the likelihood functions . . . . . . . . . 10

2.3.2 Analysis of the relations between the log-likelihood functions . . . 13

2.3 .3 Asymptotics and consistency . . . . . . . . . . . 15

2.4 On the asymptotic bias . . . . . . . . . . . . . . . . 17

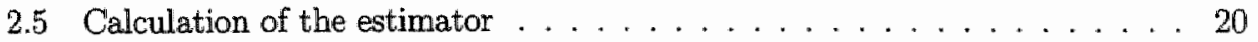

2.6 Examples 1: the AR(1) model. . . . . . . . . . . . . . . 22

2.7 Examples 2: the AR(2) model. . . . . . . . . . . . . . . 25

2.8 Which estimator to use? . . . . . . . . . . . . . 27

2.9 Conclusions . . . . . . . . . . . . . . . . . . . . . . . . . . 28

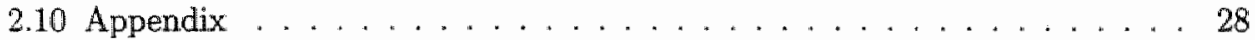

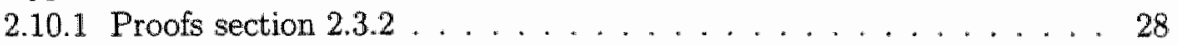

2.10 .2 Proofs section $2.3 .3 \ldots \ldots \ldots \ldots \ldots \ldots \ldots \ldots$

3 To Pool or not to Pool 37

3.1 Introduction . . . . . . . . . . . . . 37

3.2 The model and the forecasting procedures $\ldots \ldots \ldots \ldots \ldots$

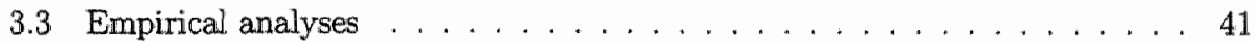

3.3.1 The models . . . . . . . . . . . . . . . . . 42

3.3.2 Properties of pooling restriction tests . . . . . . . . . 47

3.3 .3 Analyses of international data . . . . . . . . . . 51

3.4 Conclusions . . . . . . . . . . . . . . . . . . . 58

3.5 Data appendix . . . . . . . . . . . . . . . 60

4 Multiple Vector Autoregressions 63

4.1 Introduction . . . . . . . . . . . . . . . 63

4.2 The models . . . . . . . . . . . . . . . . . . . . 64 
4.3 Theory for single wector autoregressions . . . . . . . . . . . . 68

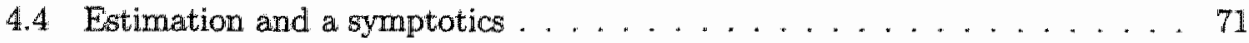

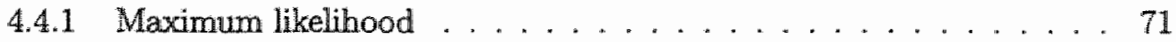

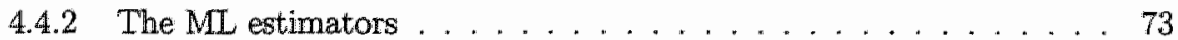

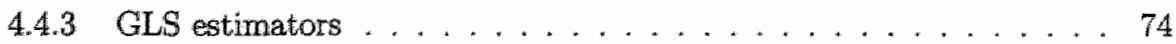

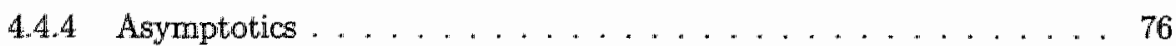

4.5 Testing. . . . . . . . . . . . . . . . . . . . . . . . . . . . . . . . . . . . . . . . .

4.6 Impulse response analysis ..................... 79

4.6.1 Nonorthogonalized impulse responses . . . . . . . . . . . . . . . 79

4.6 .2 Orthogonalized impulse responses ............... 80

4.7 An application: A small macroeconomic analysis . . . . . . . . . . 82

4.7 .1 The empirical model . . . . . . . . . . . . . . . 82

4.7 .2 Impulse response analysis ................. 84

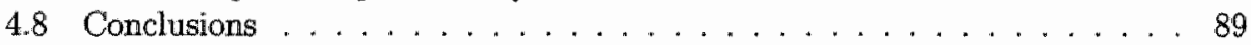

4.9 Appendix A: Technical results ... . . . . . . . . . . . . . . . . . . . . . . . . . . . . . . . . . .

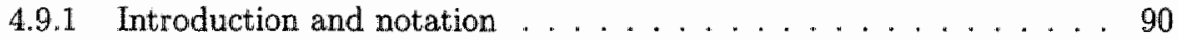

4.9.2 Proofs of Theorems 14 and $15 \ldots \ldots . \ldots 91$

4.9.3 Proofs of Theorems 16 and $17 \ldots \ldots 5$

5 Saving, Openness and Growth 103

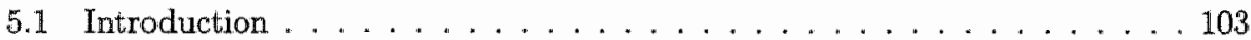

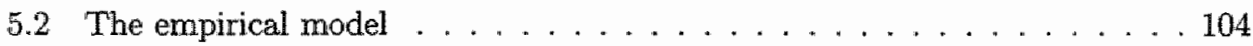

5.3 Econometric considerations . . . . . . . . . . . . . . . 107

5.3 .1 Testing for the existence of a threshold . . . . . . . . . . . 107

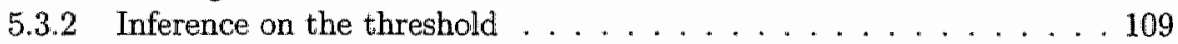

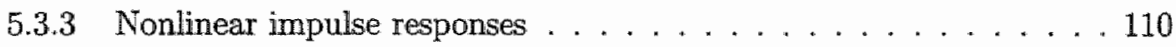

5.4 The data . . . . . . . . . . . . . . . . . . . . . . . . . . . . . . . . . . . .

5.5 Empirical Results . . . . . . . . . . . . . . . . . . . 112

5.5 .1 Long-run effects . . . . . . . . . . . . . . . . . . . . . . . . . . . . . . . . . . . .

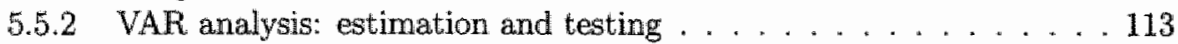

5.5 .3 VAR analysis: Impulse responses . . . . . . . . . . . 118

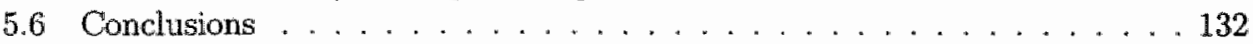

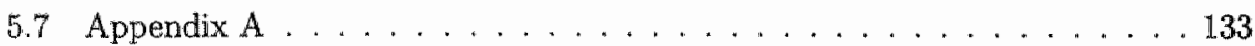

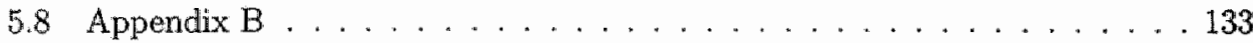

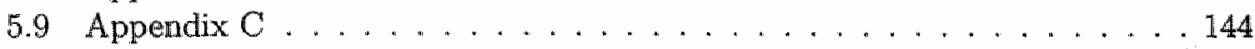

6 Conclusions $\quad 151$

6.1 Introduction . . . . . . . . . . . . . . . . 151

6.2 Summary of the results . . . . . . . . . . . . . . . . . . . . . . . . . . . . . .

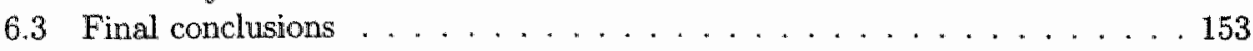

$\begin{array}{ll}\text { Bibliography } & 155\end{array}$

$\begin{array}{lr}\text { Nederlandse samenvatting } & 163\end{array}$

$\begin{array}{lr}\text { Curriculum vitae } & 167\end{array}$ 


\section{List of Figures}

2.1 Biases in the $\mathrm{AR}(1)$ model. . . . . . . . . . . . . . . . . 19

3.1 Empirical distribution of the simulated F-statistic (3.13) under parameter homogeneity. . . . . . . . . . . . . . . . 49

3.2 Empirical distribution of the simulated F-statistic (3.13) under parameter heterogeneity. . . . . . . . . . . . . . . 50

3.3 Distribution of the simulated differences between the medians of the RMSFEs (unpooled minus pooled) under parameter homogeneity. . . . . . . . 53

3.4 Distribution of the simulated differences between the medians of the RMSFEs (unpooled minus pooled) under parameter heterogeneity. . . . . . 54

4.1 Nonorthogonalized impulse responses for the Netherlands based on the $\Omega^{\text {sb }}$ model.

4.2 Orthogonalized impulse responses for the Netherlands based on the $\Omega^{\text {sh }}$ model.

4.3 Nonorthogonalized impulse responses for the Netherlands based on the individual data.

4.4 Orthogonalized impulse responses for the Netherlands based on the individual data.

5.1 Distribution of the data.

5.2 Linear impulse response functions for the model with homoscedastic innovations.

5.3 Linear impulse response functions for the model with heteroscedastic inno vations.

5.4 Impulse responses to a unit shock in openness, assuming heteroscedastic innovations. Unconditional on the regime. . . . . . . . . . 122

5.5 Impulse responses to a unit shock in openness, assuming heteroscedastic innovations. Conditional on being in the upper regime.

5.6 Impulse responses to a unit shock in openness, assuming heteroscedastic innovations. Conditional on being in the lower regime.

5.7 Average impulse responses to a unit shock in openness with 2 standarddeviation confidence bounds. Model with heteroscedastic innovations. Unconditional on regime. 
5.8 Average impulse responses to a unit shock in openness with 2 standarddeviations confidence bounds. Model with heteroscedastic innovations. Conditional on being in upper regime.

5.9 Average impulse responses to a unit shock in openness with 2 standarddeviations confidence bounds. Model. with heteroscedastic innovations. Conditional on being in lower regime. . . . . . . . . . . . . . 127

5.10 Impulse responses to a unit shock in openness for Japan based on the model with heteroscedastic innovations.

5.11 Average impulse response to a unit shock in openness for Japan. Model with heteroscedastic innovations. . . . . . . . . . . . . . . . 129

5.12 Impulse responses to a unit shock in openness for the USA based on the model with heteroscedastic innovations.

5.13 Average impulse response to a unit shock in openness for the USA. Model with heteroscedastic innovations.

5.14 Impulse responses to a one standard-deviation shock in openness assuming homoscedastic innovations. Unconditional on the regime. . . . . . . . . 134

5.15 Impulse responses to a one standard-deviation shock in openness assuming homoscedastic innovations. Conditional on being in the upper regime. . . , 135

5.16 Impulse responses to a one standard-deviation shock in openness assuming homoscedastic innovations. Conditional on being in the lower regime. . . 136

5.17 Average impulse response to a one standard-deviation shock in openness with 2 standard deviation confidence bounds. Model with homoscedastic innovations. Unconditional on regime. . . . . . . . . . . . . . . 137

5.18 Average impulse response to a one standard-deviation shock in openness with 2 standard deviation confidence bounds. Model with homoscedastic innovations. Conditional on being in upper regime. . . . . . . . . . 138

5.19 Average impulse response to a one standard-deviation shock in openness with 2 standard deviation confidence bounds. Model with homoscedastic innovations. Conditional on being in lower regime.

5.20 Impulse responses to a one standard-deviation shock in openness for Japan based on the model with homoscedastic innovations.

5.21. Average impulse response to a one standard-deviation shock in openness for Japan. Model with homoscedastic innovations.

5.22 Impulse responses to a one standard-deviation shock in openness for the USA based on the model with homoscedastic innovations.

5.23 Average Impulse response to a one standard-deviation shock in openness for the USA. Model with homoscedastic innovations. 


\section{List of Tables}

2.1 Results for the MLE in the nontransformed model, case 1. . . . . . . . . 23

2.2 Results for the MLE in the first differenced model, case 1. . . . . . . . 23

2.3 Results for the MLE in nontransformed model, case 2. . . . . . . . . . 24

2.4 Results for the MLE in the first differenced model, case 2. . . . . . . . . 24

2.5 Results for the MLE in nontransformed model, $\operatorname{ar}(2) . \ldots . . . . . .26$

2.6 Results for the MLE in the first differenced model, $\operatorname{ar}(2) . \ldots 26$

2.7 Root mean squared errors as found in the simulations. . . . . . . . . . 27

3.1 The root mean square forecast error for the individual country forecast and for the pooled forecast using the AR(3) model when up to 15 observations are excluded from the estimation period. . . . . . . . . . . . . 43

3.2 Eighteen countries 'RMSFEs of one-year-ahead forecasts, 1974-1987, 1974 1990 and 1983-1990. . . . . . . . . . . . . . . . . . . 44

3.3 Eighteen countries 'RMSFEs of one-year-ahead forecasts, 1974-1987, 1974 1990 and $1983-1990 . \ldots \ldots \ldots \ldots \ldots$

3.4 Testing for contemporaneous error covariances. . . . . . . . . . . . . . . 46

3.5 Testing for pooling for a subset of 6 countries (Canada, USA, Belgium, France, Germany, Netherlands) . . . . . . . . . . . . . . . . 48

3.6 Rejection frequencies of the F-test (3.13) at a 5 significance level. . . . . . 51

3.7 Mean and Median (Median)RMSFE for the simulations. . . . . . . . . 52

3.8 Testing for pooling in the AR(3)LI model. . . . . . . . . . . . . . . . 56

3.9 Testing for pooling in the AR(3) model. . . . . . . . . . . . . 57

3.10 Root mean square forecast errors for the AR(3)LI and AR(3) models. . . 59

3.11 Observations available per country. . . . . . . . . . . . . . 61

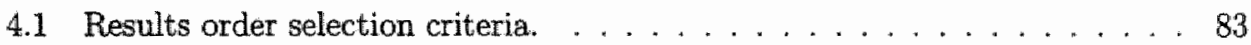

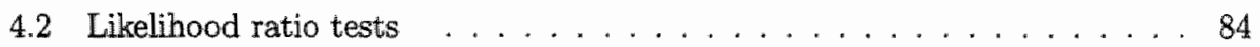

5.1 Tests for the existence of a threshold. . . . . . . . . . . . . 113

5.2 Tests for unit roots. . . . . . . . . . . . . . . . . . . . . 114

5.3 Estimation results linear VAR under homoscedasticity. . . . . . . . . 115

5.4 Testing for the existence of a threshold in the VAR under homoscedasticity. 115

5.5 Estimation results for the threshold model under homoscedasticity. . . . 116

5.6. Estimation results for the linear VAR under heteroscedasticity. . . . . . 117

5.7 Testing for the existence of threshold in the VAR under heteroscedasticity. 117

5.8 Estimation results for the threshold model under heteroscedasticity. . . . . 118 
5.9 Estimation results for VAR with homoscedastic innovations, with no cross-

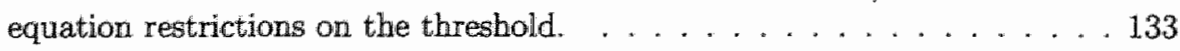




\section{Chapter 1}

\section{Introduction}

\subsection{Introduction}

The joint analysis of dynamic models for a number of entities is the leading thread running through this thesis. The focus is on estimation and inference in dynamic models in which the entities or individuals are assumed to have certain common characteristics. Such models have become an important tool in studying macroeconomic problems which raise questions on differences or similarities between countries or regions.

The analysis of dynamic models where the number of individuals is small relatively to the number of observations through time, referred to as time series analysis, has a long history and is well established by now. The analysis of dynamic models with a large number of individuals observed over a small number of time periods, referred to as panel data analysis, is of a more recent date. Estimation and inference in panel data models appeared to be more complicated as some commonly used analysis techniques were found to be unsuitable for analysis of such models. In recent years, however, these problems have been studied by numerous researchers and solutions have been presented or suggested.

A problem often encountered in practice is how to deal with data sets that have dimensions which allow for both panel data analysis and time series analysis: which type of analysis is more efficient and more appropriate? A related problem arises when data sets have too few individuals to be analyzed accurately as panel data and too few observations through time to be analyzed accurately as time series. How to model and analyze the available information with sufficient accuracy?

To study the above and other associated problems the Economics Research Foundation, which is part of the Netherlands Organization for Scientific Research (NWO) funded a research program in the field of panel data, of which the project entitled "Nonlinear models for panel data: Methodological aspects and applications to forecasting international business cycle data" is part. This thesis collects the research output of 4 years of research on this project.

This thesis particularly focuses on situations in which there are a fixed number of individuals and a moderately large number of observed time periods. In the literature such data sets are used to address problems in different fields of economic research. In finance for instance, the analysis of the term structure of interest rates, done by Bilson (1981), Mankiw (1986); in international macroeconomics, studies on convergence by Bernard and 
Durlauf (1995), Mankiw, Romer and Weil (1992), Islam (1995) and Nerlove (1996); on forecasting GDP-growth rates, Garcia-Ferrer et al.(1987), Zellner and Min (1991) and Zellner and Hong (1989); on forecasting gasoline demand, Baltagi and Griffin (1983, 1997); and in marketing by Blattberg and George (1991).

\subsection{Dynamic panel data models}

The analysis of panel data sets has become an important research area in econometrics. Numerous studies, theoretical as well as applied, have been published. For an extensive review on the use and analysis of panel data in econometrics, the reader is invited to consult Hsiao (1986), Baltagi (1995) and Mátyás and Sevestre (1996). These books give a broad insight in the different theoretical issues and summarize several applications. They also include an extensive list of references to articles on almost all subjects related to the analysis of panel data.

In this thesis the focus is on the analysis of models denoted in the literature by the term 'dynamic panel data models'. These models are characterized by the fact that current individual behavior is explained, among other things, by past behavior of the same individual and by common behavior of the group the individual is part of.

Dynamic panel data models can be evaluated using panel data as well as time series analysis techniques. The difference is that in panel data analysis it is usually assumed that the model holds for a finite number of time periods and a large number of individuals. In time series analysis it is assumed that the reverse holds.

When an large number of observations, in either of the dimensions, would be available there would be no question about the choice between time series or panel data analysis. In practice this still holds true if one of either dimensions is much larger than the other. In macroeconomic applications, the field of application in this thesis, we usually have a fixed and given number of individuals and a growing number of observed time periods. However, it often happens that available data sets do not have the property that the number of observed time periods is substantially larger than the number of individuals. In such cases the same model, based on the observed number of time periods and individuals, could well be analyzed both by panel data analysis as well as a time series analysis techniques. The choice is left in the hands of the researcher.

This would of course be no problem if the two different types of analysis, which only differ in the assumed magnitude of dimensions, lead to the same answer. This, however, is not always the case. As a result one has first to resolve the issue which framework of analysis is appropriate. Should one rely on large $T$ asymptotics (time series framework) or should one use large $N$ asymptotics (panel data framework), or is a dynamic panel framework appropriate with both $N$ and $T$ being large? In many applications it is difficult to settle this issue. We consider this problem for one model only, the panel data AR(p) model. This analysis can be found in chapter two.

In the chapters three, four and five we chose for time series models i.e. we assumed $T$ be large. This choice was made on grounds of the problems at hand and the fact that in our opinion the choice of appropriate asymptotics can only be solved by simulation of theoretical models and not by empirical models. It is clear that more research is needed 
to give clear-cut answers to the problem of the choice of the appropriate asymptotic assumptions, but this lies beyond the scope of this thesis.

While modelling several individuals jointly one can ask whether some or all of the parameters for the different individuals should be considered deterministic or random. When parameters are assumed to be random, the parameters in the model for an individual are realizations from a random draw from a population. If some parameters in the model are assumed to be random it is usually referred to as a random coefficient model.

We do not consider these models because in macroeconomics very often nonrandom parameter models are used. The motivation is twofold: First, a typical macro panel most likely contains all the individuals of interest and not just a random subgroup from a larger population of individuals. For instance when studying an OECD panel it usually contains all OECD countries or a specific subgroup thereof, and not a random sample of them. Second, if the random parameters should capture individual specific effects it is very likely that these parameters are correlated with the regressors. For the same reasons we do not consider random effects panel data models. In these models only the individual effects are considered random. For a further discussion on the choice between fixed and random effects we refer to Mundlak (1978) and Hsiao (1986) section 3.4.

When analyzing dynamic panel data models there are several estimators available to base inference on. The most prominent ones are Maximum Likelihood (ML), Least Squares (LS), Instrumental Variables (IV) and the more recently developed extension of IV, Generalized Method of Moments (GMM). In this thesis we confine our attention to ML and LS estimation. Under the assumption of normality these two methods often lead to the same estimator, see for instance Hamilton (1994). In chapter two, however, we encounter a case in which the two do not coincide. The advantage of ML is that, when it is consistent, it leads to asymptotically efficient estimators. The simplicity of LS makies it a very popular estimator which, under the assumption of normality, is also efficient when it coincides with $\mathrm{ML}$.

We do not consider IV and GMM estimators. IV and GMM estimators are usually less efficient than ML as they in general do not take into account all the moment conditions implied by the model, see e.g. Wansbeek and Bekker (1996). Efficient GMM, which uses all the implied moment conditions is difficult to calculate when $T$ becomes large due to the large matrices containing all the instruments. Further it is often found in small sample property studies that IV and GMM show an undesirably high variability, see e.g. Kievit. (1995) and Judson and Owen (1997).

An advantage of GMM over ML estimators is that they are more robust to misspecification. However, taking into account the arguments of the two previous paragraphs we prefer ML to IV and GMM estimators. ML is both efficient and still computable within reasonable time.

\subsection{Outline}

In chapter two we give a brief introduction to the problems associated with the analysis of dynamic panel data models. In particular we focus on differences in asymptotic behavior of Maximum Likelihood Estimators under the assumption of large $N$ asymptotics, assuming 
the number of individuals to be large, and large $T$ asymptotics, assuming the number of observed periods to be large. In dynamic panel data models the choice of either large $\mathrm{N}$ or large $\mathrm{T}$ asymptotics has important implications for the assumed asymptotic behavior of the estimators.

In particular we study one of the most disturbing problems in dynamic panel data analysis: The inconsistency of the Maximum Likelihood Estimator when the number of observed time periods is small. It is already known that one can consistently estimate dynamic panel data models applying MLE to a transformation of the model. We derive the asymptotic distribution of the MLE in the transformed and non-transformed model under different assumptions on $N$ and $T$. The transformed model expresses the variables in first differences. Furthermore, we extend some well-known results for the AR(1) model to $A R(p)$ panel data models. We derive a first order approximation for the bias of the $M L$ estimator in the non-transformed AR(p) models. This result might be used to develop a bias corrected estimator in the non-transformed $\operatorname{AR}(\mathrm{p})$ panel models. A simulation study evaluates the theoretical results. In the simulation the Maximum Likelihood estimator based on the transformed model outperforms the Maximum Likelihood estimator based on the non-transformed model using the Mean Squared Error as criterion.

In chapter three we investigate whether pooling can improve one-step-ahead forecasting of international output growth rates. This subject has been studied in various contributions, often in the context of shrinkage estimators. Our approach to this problem starts from the SURE (seemingly unrelated regressions equations) procedure introduced by Zellner (1962). We investigate whether tests on poolability such as the standard Chow (1960) test or less restrictive tests like the second weak mean square error criterion of McElroy (1977) give a good indication whether pooling improves forecasting. The second weak mean squared error criterion compares the pooled estimator to the individual estimator in terms of a quadratic form of the predicted value to the conditional mean, the weighting matrix of the quadratic form being the inverse of the covariance matrix of the disturbance term.

To empirically evaluate the power of the indicative tests we analyze an autoregressive leading indicator model for 18 OECD countries. We allow for correlations between individual countries. Blocks of correlated countries are identified and subsequently used in prediction. We evaluate the performance of the different tests on poolability in connection with the performance of the resulting one-step-ahead forecasts. In the simulation it is found that these tests, especially the second weak mean square error criterion, give a reasonable indication on whether pooling improves forecasting.

The research in chapter three is joint work with Franz Palm and Gerard Pfann both from Maastricht University. The chapters four and five are based on research initiated while I was visiting the Southern Methodist University (SMU) in Dallas. Chapter five is joint work with Thomas Osang from the SMU.

In chapter four a class of Vector Autoregressive models is introduced. Sets of restrictions across individuals, often used in the panel data literature, are studied. The models are intended to parsimoniously parametrize multivariate time series for a fixed number of individuals with the aim to efficiently estimate their parameters. Assuming a common lag structure but individual means and decomposing the innovation covariance matrix in individual specific and common parameters, efficiency, as compared with the 
unrestricted individual models, is gained. The asymptotic distribution for the Maximum Likelihood estimator under these sets of restrictions is derived analytically and used to obtain analytical confidence bounds for impulse response functions of the VAR-models.

To demonstrate the possibilities of the use of the cross-individual restrictions in macroeconometric analysis we perform an impulse response analysis for six OECD countries. Using a trivariate VAR, with GDP, money supply and inflation as variables, we find indeed that imposing the restrictions leads to a gain in precision. Based on the estimates of the six OECD countries we illustrate the efficiency gain by giving a detailed analysis of the orthogonalized impulse responses for the Netherlands.

In chapter five we study the relation between a country's savings rate, openness to trade and economic growth. The analysis is performed for a panel of 58 countries. We analyze a dynamic multiwariate threshold model with an endogenously determined threshold. The threshold variable is a country's savings ratio to GDP. Countries are allowed to switch from a high savings regime to a low savings regime over time. A new test is developed for the existence of an endogenously determined threshold. For the 58 countries we identify the threshold and analyze the short-term dynamic behavior of the model at its equilibrium by means of impulse response analysis.

Using nonlinear impulse response function analysis we find significantly different reactions of countries to an unanticipated shock in openness depending on the savings rate two years before the time of the shock. This is illustrated by analyzing the responses for the USA and Japan.

Finally, in chapter six we present a summary of the conclusions reached in the previous chapters. 
CHAPTER 1. INTRODUCTION 


\section{Chapter 2}

\section{Maximum likelihood estimation in dynamic panel data models}

\subsection{Introduction}

In modern economics there are many research questions which ask for an analysis of $\mathrm{dy}$ namic models across several units or individuals, for instance countries, regions, industrial sectors etc. The data available in many of these studies often have dimensions which are neither typical for panel data analysis, because the number of individuals is too small $(10<N<100)$, nor for time series analysis, since the time dimension is usually too small $10<T<30$. Therefore, when modelling dynamics in the data, the choice between the assumptions (1) $T \rightarrow \infty, N$ fixed, (2) $N \rightarrow \infty, T$ fixed and (3) $T \rightarrow \infty, N \rightarrow \infty$ to derive the asymptotic properties is not always obvious.

The choice between the assumptions determines the choice of the estimator. In this chapter, where we consider a fixed effects dynamic panel data model, the Maximum Likelihood Estimator (MLE) is consistent and gives the same asymptotic inference results when $T \rightarrow \infty$, i.e. for the situations (1) and (3). However for (2), i.e. $N \rightarrow \infty, T$ finite, the MLE is inconsistent because the individual effects form incidental parameters. For panel data, especially larger panels, this fact complicates estimation and inference. For references on these, by now classic, results we refer to Nickell (1981), Sevestre and Trognon (1985), Kievit (1995), Hsiao (1986) and Baltagi (1995), the latter two being more general introductions into panel data analysis.

When conditioned on the initial observations, the MLE (which under this assumption is identical to Least. Squares Dummy Variable Estimation (LSDV)) is inconsistent for finite $T$. Therefore, several other, consistent, methods have been studied. Most prominent are IV or GMM methods. First the data are differenced once to eliminate the additive individual effects ${ }^{1}$ and then instruments are constructed to estimate the model. In this context we refer to Anderson and Hsiao (1982), Arellano and Bond (1991), Arellano and Bover (1995), Ahn and Schmidt (1995, 1997), Amemiya and MaCurdy (1986) and Breusch, Mizon and Schmidt (1989).

\footnotetext{
${ }^{1}$ Other ways of eliminating the individual effects, which we will not explore in this chapter, take all data in deviation from a base period or in deviation of their sample means.
} 
In the remaining part of this chapter we often use the term "first differenced model". We use this term to denote the model for the data in first differences with the stochastic properties as implied by the model in levels, i.e. the model for the data in first differences of an AR panel data model has disturbances which are MA(1) with a unit root.

The reason for using IV estimators in the first differenced models is that the MLE/LSDV is inconsistent when $T$ is kept finite. However, as Lancaster and Lindenhovius (1996) recently showed, exact MLE applied to the first differenced model is consistent in the AR(1) case with random initial observations. The reason why this result was obtained only recently may be that OLS and MLE do not coincide in this case. The search for likelihood based consistent estimators is driven by the possibility to conduct asymptotic inference. Moreover, it has been found in practice that many IV or GMM estimators exhibit an undesirable high variability.

In this chapter the consistency of the ML estimator in the first differenced model is extended from the $\mathrm{AR}(1)$ to the $\mathrm{AR}(\mathrm{p})$ model. We derive the asymptotic distributions of the ML estimator in the first differenced model. Using the relation between the ML estimator in the first differenced AR(p) model and the ML estimator in the non-transformed direct AR(p) model we give explicit formula for asymptotic biases of the ML estimator for the non-transformed panel data AR(p) model. We analyze the ML estimators in the non-transformed and first differenced model under the three possible types of asymptotics regarding $N$ and $T$.

This chapter is organized as follows. In section 2 we give a short overview of known results linked to the topic of this chapter and a further motivation for this chapter. In section 3 we discuss likelihood analysis of AR(p) models in dynamic panel data and establish asymptotic distributional results. In section 4 we derive a first order approximation of the bias of MLE applied to the non-transformed model. In the sections 5 and 6 we illustrate the results by analyzing AR(1) and AR(2) models and report on a small sample size simulation to evaluate how well the derived asymptotic results hold under different sample sizes of $N$ and $T$. The main conclusions are drawn in section 7 .

\subsection{Analysis of dynamic panel data}

In this section we put the results presented in this chapter in perspective with respect to the existing literature on the estimation of dynamic panel data models. We focus on the fixed effects dymamic panel data model and do not consider the random effects dynamic panel data model. The same problem, i.e. the inconsistency of estimators as OLS and GLS in dynamic panel data models, also appears in the random effects case, see for instance Trognon (1978) and Sevestre and Trognon (1985). For a review see Ridder and Wansbeek (1990).

In the literature there is a large number of studies dealing with the following simple dynamic fixed effects model

$$
\begin{aligned}
& y_{i, t}=c_{i}+\phi_{1} y_{i, t-1}+\varepsilon_{i, t}, \\
& \varepsilon_{i, t} \sim \operatorname{NID}\left(0, \sigma^{2}\right),
\end{aligned}
$$

where $t=1, \ldots, T, i=1, \ldots, N$. It is well known that the LSDV and ML estimator of $\phi_{1}$ is 
biased and inconsistent for finite $T$. This was shown by Nickell (1981) for the LSDV in the case of stochastic initial conditions. He derived an explicit formula for the bias which was shown to be of order $T^{-1}$. The bias is mainly due to the fact that these estimators do not eliminate the infinitely many incidental parameters $c_{\text {i }}$ from the model without introducing correlations in the time dimension. Only when we have an infinite time dimension these correlations can be neutralized.

In attempts to obtain consistent estimators for $\phi_{1}$ the first differenced model was studied

$$
\left(y_{i, t}-y_{i, t-1}\right)=\phi_{1}\left(y_{i, t-1}-y_{i, t-2}\right)+\left(\varepsilon_{i, t}-\varepsilon_{i, t-1}\right)
$$

However, this did not bring a direct solution to the problem of inconsistency of the usual estimators like OLS and GLS. The incidental parameters are eliminated, but the correlations between regressors and disturbances again make the OLS estimator for $\phi_{1}$ inconsistent as $N \rightarrow \infty$ but $T$ is kept fixed; this was for instance noticed by Chamberlain (1980). Using a transformation of the OLS estimator we can estimate $\phi_{1}$ consistently, but the estimator will not be efficient.

Consistent estimators in the first differenced model, GMM and IV estimators, were intraduced, first by Anderson and Hsiao (1982), later by many others, see for instance: Arellano and Bond (1991), Arellano and Bover, (1995), Ahn and Schmidt (1995) and (1997), Amemiya and MaCurdy (1986) and Breusch, Mizon and Schmidt (1989). These estimators, although consistent and efficient within certain classes of estimatiors, appeared in practice to suffer from relative high variability, even when $T$ is relatively large, see for instance Kievit (1995).

This led to the introduction of the MLE or LSDV with bias corrections based on the non transformed model (2.1) as these estimators show in practice much less variability. The most promising one is given by Kievit (1995) to whom we also refer for further references on other bias correction methods. In small sample size studies the bias corrected LSDV as proposed by Kievit outperforms the GMM/IV type of estimators in RMSE sense.

Lancaster and Lindenhovius (1996) showed that ML estimation of the above first differenced model is consistent when assuming stochastic initial conditions. This result implies that we can estimate efficiently the parameter $\phi_{1}$ without the need for a bias correction. As will be shown, a disadvantage of the ML estimator in the first differenced dynamic panel data model is that it is not consistent when we condition on the initial observations.

Another important question is how to conduct inference and efficient estimation in data sets where both $N$ and $T$ are moderately large, so that it is not obvious which type of asymptotics is most appropriate. In that case it is still an open question how the estimators perform in practice. Therefore we conduct in this chapter a thorough analysis of MLE in the above model with the assumptions on $N$ and $T$ varying.

The above discussion has centered on the AR(1) fixed effects dynamic panel data model. In this chapter however we analyze the more general AR(p) model. In this way we generalize the result of Nickell (1989), and give an alternative estimator to the GMM/IV, LSDV or bias corrected LSDV methods in fixed effect dynamic panel data models. 


\subsection{Likelihood analysis of $\mathrm{AR}(\mathrm{p})$}

\subsubsection{Derivation of the likelihood functions}

We discuss the following simple second order stationary dynamic fixed effects model

$$
y_{i, t}=c_{i}+\phi_{1} y_{i, t-1}+\ldots+\phi_{p} y_{i, t-p}+\varepsilon_{i, t}, \quad \varepsilon_{i, t} \sim N I D\left(0, \sigma^{2}\right),
$$

where $i=1, \ldots, N$ and $t=1, \ldots, T$. We derive for this model and the associated model for the first differenced data the log-likelihoods. The likelihoods for both models are considered conditionally on as well as jointly with the initial observations. We later use the relations between the different log-likelihoods to derive the bias of the ML estimator in model (2.4).

Assume we have observed $y_{i}=\left(y_{i,-p+1}, \ldots, y_{i, 0}, \ldots, y_{i, T}\right)^{\prime}, i=1,2, \ldots, N$, where the observations $y_{i,-p+1}, \ldots, y_{i, 0}$ are the initial observations. To identify all the parameters in an AR(p) model we need at least $p+2$ observations for each $i$. In the remaining part of the chapter where we also consider the first differenced model of (2.4) we assume to have observed at least $p+3$ observations. This enables us to identify and estimate all parameters in both the direct model and the first differenced model, including the variance of the innovation.

Denote the covariance matrix of a $\mathrm{AR}(\mathrm{p})$ process with $t$ observations $(t \geq p)$ by $\sigma^{2} V_{t}^{p}$. Let $\mu_{i}$ be the unconditional expectation of time series for individual $i$, i.e. $\mu_{i}=E\left(y_{i, t}\right)$ then we have

$$
\mu_{i}=\frac{c_{i}}{1-\phi_{1}-. .-\phi_{p}} .
$$

Using the autocovariance of the stationary series $y_{i, t}, \gamma_{s}=E\left(y_{i, t}-\mu_{i}\right)\left(y_{i, t+s}-\mu_{i}\right)$ we notice

$$
\sigma^{2} V_{t}^{p}=\left[\begin{array}{ccccc}
\gamma_{0} & \gamma_{1} & \gamma_{2} & \ldots & \gamma_{t-1} \\
\gamma_{1} & \gamma_{0} & \gamma_{1} & \gamma_{2} & \cdot \\
\gamma_{2} & \gamma_{1} & \gamma_{0} & \gamma_{1} & \gamma_{2} \\
\cdot & \gamma_{2} & \gamma_{1} & \gamma_{0} & \gamma_{1} \\
\gamma_{t-1} & . . & \gamma_{2} & \gamma_{1} & \gamma_{0}
\end{array}\right]
$$

Observe that the model (2.4) implies $\sigma^{2} V_{t}^{p}$ to be the same for every individual. We analyze this model under two different initial conditions. In the first case we assume the initial observations to be stochastic and we assume the marginal distribution of the initial $p$ observations $y_{i,-p+1}, \ldots, y_{i, 0}$ to be given by

$$
\left(y_{i,-p+1}, . ., y_{i, 0}\right)^{\prime} \sim N\left(\mu_{i}, \sigma^{2} V_{p}^{p}\right) .
$$

This assumption on the initial observations is consistent with a stationary $A R(p)$ model. It is the unconditional distribution of the initial observations given that the process has been operating from $-\infty$ on. In the second case we condition on the initial observations $y_{i,-p+1}, \ldots, y_{i, 0}$, i.e. we assume them to be fixed.

Observe that we can rewrite (2.4) also in mean-adjusted form

$$
\left(y_{i, t}-\mu_{i}\right)=\phi_{1}\left(y_{i, t-1}-\mu_{i}\right)+\ldots+\phi_{p}\left(y_{i, t-p}-\mu_{i}\right)+\varepsilon_{i, t}, \quad \varepsilon_{i, t} \sim N I D\left(0, \sigma^{2}\right),
$$


$t=1, \ldots, T$. It is well known that in case (2.7) holds for the initial observations, the distribution of $\left(y_{i}-\mu_{i} \iota_{T+p}\right)$ is given by

$$
\left(y_{i}-\mu_{i} \iota_{T+p}\right) \sim N\left(0, \sigma^{2} V_{T+p}^{p}\right)
$$

where $\iota_{x}$ is a vector of $x$ ones. The log-likelihood function of $y_{i}$ is now given by

$$
\begin{aligned}
l^{J}= & -\frac{N(T+p)}{2} \log (2 \pi)+\frac{N}{2} \log \left|\sigma^{-2}\left(V_{(T+p)}^{p}\right)^{-1}\right| \\
& -\frac{1}{2} \sum_{i=1}^{N}\left(y_{i}-\mu_{i} \iota_{T+p}\right)^{\prime}\left[\sigma^{-2}\left(V_{(T+p)}^{p}\right)^{-1}\right]\left(y_{i}-\mu_{i} \iota_{T+p}\right) .
\end{aligned}
$$

The superscript $J$ indicates that the likelihood is derived under the assumption of stochastic initial observations and it is the joint log-likelihood of initial and further observations. Using the prediction-error decomposition of the log-likelihood [see for instance Hamilton (1994), page 122-124] we obtain

$$
\begin{aligned}
l^{J}= & -\frac{N p}{2} \log (2 \pi)+\frac{N}{2} \log \left|\sigma^{-2}\left(V_{(p)}^{p}\right)^{-1}\right| \\
& -\frac{1}{2} \sum_{i=1}^{N}\left(y_{i, 0}-\mu_{i}\right)^{\prime}\left[\sigma^{-2}\left(V_{(p)}^{p}\right)^{-1}\right]\left(y_{i, 0}-\mu_{i} l_{p}\right) \\
& -\frac{N T}{2} \log (2 \pi)+\frac{N T}{2} \log \left|\sigma^{-2}\right| \\
& -\frac{1}{2 \sigma^{2}} \sum_{i=1}^{N} \sum_{t=1}^{T}\left(\left(y_{i, t}-\mu_{i}\right)-\phi_{1}\left(y_{i, i-1}-\mu_{i}\right)-\ldots-\phi_{p}\left(y_{i, t-p}-\mu_{i}\right)\right)^{2}
\end{aligned}
$$

where $y_{i, 0}=\left(y_{i,-p+1}, . ., y_{i, 0}\right)^{\prime}$. It follows directly that in case we condition on the initial observations the likelihood function is given by

$$
\begin{aligned}
& l^{C}=-\frac{N T}{2} \log (2 \pi)+\frac{N T}{2} \log \left|\sigma^{-2}\right| \\
& -\frac{1}{2 \sigma^{2}} \sum_{i=1}^{N} \sum_{t=1}^{T}\left(\left(y_{i, t}-\mu_{i}\right)-\phi_{1}\left(y_{i, t-1}-\mu_{i}\right)-\ldots-\phi_{p}\left(y_{i, t-p}-\mu_{i}\right)\right)^{2} .
\end{aligned}
$$

The superscript $C$ denotes the fact that the log-likelihood is conditional on the initial observations.

To eliminate the incidental parameters $c_{i}$ in (2.4) we need to transform the model. Transforming the data in deviations from their individual mean introduces correlations between the regressors and errors which renders MLE or LSDV inconsistent. Taking the data in deviation from a base period makes the analysis dependent on this base period which is also undesirable. Therefore we consider the model for the variables expressed in first differences

$$
\left(y_{i, t}-y_{i, t-1}\right)=\phi_{1}\left(y_{i, t-1}-y_{i, t-2}\right)+\ldots+\phi_{p}\left(y_{i, t-p}-y_{i, t-p-1}\right)+\varepsilon_{i, t}-\varepsilon_{i, t-1} .
$$

where $t=2, \ldots, T$. Observe that if one would apply a regression analysis this directly implies a loss of information due to the fact that we lose one observation. 
To simplify the analysis we introduce the following two matrices: A matrix containing the coefficients of the $A R(p)$ model

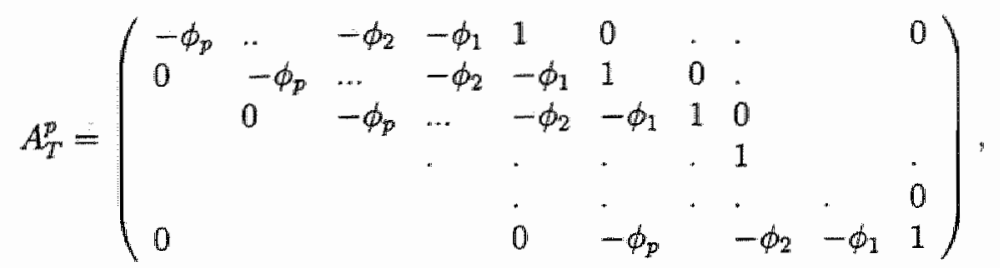

with $\operatorname{dim}\left(A_{T}^{p}\right)=T \times(T+p)$ and the matrix which transforms a $(T \times 1)$ vector in a $((T-1) \times 1)$ vector of first differences

$$
D_{T}=\left(\begin{array}{ccccc}
-1 & 1 & & & \\
& -1 & 1 & & \\
& & & & \\
& & & -1 & 1
\end{array}\right), \quad \operatorname{dim}\left(D_{T}\right)=(T-1) \times T .
$$

Now observe that $(2.8)$ can be restated as

$$
A_{T}^{p}\left(y_{i}-\mu_{i} \iota_{T+p}\right)=\varepsilon_{i},
$$

where $\varepsilon_{i}=\left(\varepsilon_{i, 1}, \ldots, \varepsilon_{i, T}\right)^{\prime}$. If we condition on the initial observations i.e. when they are considered to be given and fixed (2.16) contains all the information on the model and we shall use this equation to derive the likelihood of the transformed model in this case. To get rid of the individual means we take the first difference of (2.16) and obtain

$$
D_{T} A_{T}^{p}\left(y_{i}-\mu_{i} \iota_{T+p}\right)=D_{T} A_{T}^{p} y_{i}=D_{T} \varepsilon_{i} .
$$

Here we used that $A_{T}^{p}$ operating on $t_{T+p}$ is a vector containing the same constants in every position and $D_{T}$ maps a constant vector into a zero vector. This means that we have

$$
D_{T} A_{T}^{p}\left(y_{i}-\mu_{i} \iota_{T+p}\right) \sim N\left(0, \sigma^{2} D_{T} D_{T}^{\prime}\right)
$$

with $\log$-likelihood function

$$
\begin{aligned}
l_{F D}^{C}= & -\frac{N(T-1)}{2} \log (2 \pi)+\frac{N}{2} \log \left|\sigma^{-2}\left(D_{T} D_{T}^{\prime}\right)^{-1}\right| \\
& -\frac{1}{2} \sum_{i=1}^{N} y_{i}^{\prime} A_{T}^{p p} D_{T}^{\prime}\left[\sigma^{-2}\left(D_{T} D_{T}^{\prime}\right)^{-1}\right] D_{T} A_{T}^{p} y_{i} .
\end{aligned}
$$

The subscript $F D$ indicates that it is the log-likelihood from the first differenced model.

When we assume the initial observations to be stochastic it follows directly from (2.9) that

$$
D_{T+p}\left(y_{i}-\mu_{i}\right)=D_{T+p} y_{i} \sim N\left(0, \sigma^{2} D_{T+p} V_{T+p}^{p} D_{T+p}^{\prime}\right)
$$

and the associated $\log$-likelihood function equals

$$
\begin{aligned}
l_{F D}^{J}= & -\frac{N(T+p-1)}{2} \log (2 \pi)+\frac{N}{2} \log \left|\sigma^{-2}\left(D_{T+p} V_{T+p}^{p} D_{T+p}^{\prime}\right)^{-1}\right| \\
& -\frac{1}{2} \sum_{i=1}^{N} y_{i}^{\prime} D_{T+p}^{\prime}\left[\sigma^{-2}\left(D_{T+p} V_{T+p}^{p} D_{T+p}^{\prime}\right)^{-1}\right] D_{T+p} y_{i} .
\end{aligned}
$$


Of course we can also factorize this log-likelihood in the marginal for the initial observations and the conditional $\log$-likelihood for the observations from $t=1$ on given the initial values. This gives

$$
\begin{aligned}
l_{F D}^{J}= & -\frac{N p}{2} \log (2 \pi)+\frac{N}{2} \log \left|\sigma^{-2}\left(D_{p+1} V_{p+1}^{p} D_{p+1}^{\prime}\right)^{-1}\right| \\
& -\frac{1}{2} \sum_{i=1}^{N} y_{i}^{* *} D_{p+1}^{\prime}\left[\sigma^{-2}\left(D_{p+1} V_{p+1}^{p} D_{p+1}^{\prime}\right)^{-1}\right] D_{p+1} y_{i}^{*} \\
& -\frac{N(T-1)}{2} \log (2 \pi)+\frac{N}{2} \log \left|\sigma^{-2}\left(D_{T} D_{T}^{\prime}\right)^{-1}\right| \\
& -\frac{1}{2} \sum_{i=1}^{N} y_{i}^{\prime} A_{T}^{p \prime} D_{T}^{\prime}\left[\sigma^{-2}\left(D_{T} D_{T}^{\prime}\right)^{-1}\right] D_{T} A_{T}^{p} y_{i} .
\end{aligned}
$$

Here $y_{i}^{* *}=\left(y_{i_{1}-p+1}, \ldots, y_{i, 1}\right)$ and $y_{i}^{\prime}=\left(y_{i_{i}-p+1}, \ldots, y_{i, T}\right)$ as before.

In the following section the above given four likelihoods $l^{J}(2.10), l^{C}(2.12), l_{F D}^{C}(2.19)$ and $l_{F D}^{J}(2.21)$ and the relations among them will be analyzed. This will be done under different assumptions on $N$ and $T$.

\subsubsection{Analysis of the relations between the log-likelihood func- tions}

The first differenced model is derived from the model given by (2.4). Therefore it is very likely that there are close relations between the various log-likelihoods of the two models as derived in the previous section. In this section we analyze these relations, we later use these relations to obtain for instance the asymptotic bias of the ML estimator in the model for the levels.

The parameters of interest are the coefficients of the lag polynomial $\phi_{1}, \ldots, \phi_{p}$ and the variance of the innovation $\sigma^{2}$. Therefore we first concentrate the log-likelihoods $l^{J}$ and $l^{c}$ with respect to the individual mean parameters.

Lemma 1 The log-likelihoods $l^{J}$ and $l^{C}$ concentrated with respect to $\mu_{i}$ are given by

$$
\begin{aligned}
\tilde{l}^{J}= & -\frac{N(T+p)}{2} \log (2 \pi)+\frac{N}{2} \log \left(\mid \sigma^{-2}\left(V_{(T+p)}^{p}\right)^{-1} \|\right) \\
& -\frac{1}{2} \sum_{i=1}^{N} y_{i}^{\prime} D_{T+P}^{\prime}\left(\sigma^{-2}\left(D_{T+p} V_{(T+p)}^{p} D_{T+p}\right)^{-1} D_{T+p} y_{i}\right.
\end{aligned}
$$

and

$$
\begin{aligned}
\tilde{l}^{C}= & -\frac{N T}{2} \log (2 \pi)+\frac{N T}{2} \log \left(\sigma^{-2}\right)- \\
& \frac{1}{2 \sigma^{2}} \sum_{i=1}^{N} \sum_{t=1}^{T}\left(\left(y_{i, t}-\bar{y}_{i, 0}\right)-\phi_{1}\left(y_{i, t-1}-\bar{y}_{i,-1}\right)-\ldots-\phi_{p}\left(y_{i, t-p}-\bar{y}_{i,-p}\right)\right)^{2}
\end{aligned}
$$

where $\bar{y}_{i,-j}=\frac{1}{T} \sum_{t=1}^{T} y_{i, t-j}$. 
The proof is given in the appendix. Before comparing the different likelihoods with each other we first give the following result for $l_{F D}^{C}$ :

Lemma 2 The log-likelihood of the first differenced madel conditional on the initial observations equals

$$
\begin{aligned}
l_{F D}^{C}= & -\frac{N(T-1)}{2} \log (2 \pi)+\frac{N(T-1)}{2} \log \left(\sigma^{-2}\right)- \\
& \frac{1}{2 \sigma^{2}} \sum_{i=1}^{N} \sum_{t=1}^{T}\left(\left(y_{i, t}-\bar{y}_{i, 0}\right)-\phi_{1}\left(y_{i, t-1}-\bar{y}_{i_{n}-1}\right)-\ldots-\phi_{p}\left(y_{i, t-p}-\bar{y}_{i_{2}, p}\right)\right)^{2},
\end{aligned}
$$

where $\bar{y}_{i_{1}-j}=\frac{1}{T} \sum_{t=1}^{T} y_{i, t-j}$.

This proof is also given in the appendix. Observe that the above Lemmas 1 and 2 imply that the ML estimators for $\phi$ based on $l_{F D}^{C}$ and $l^{C}$ or $\tilde{l}^{C}$ are identical. Maximizing the likelihoods $\tilde{l}^{C}, l_{F D}^{C}$ or $l^{C}$ all results in the LSDV estimator. This estimator has an asymptotic bias for finite $T$ even if $N \rightarrow \infty$. For the AR(1) case this asymptotic bias was derived by Nickell (1981). It is given by

$$
\begin{aligned}
& p \lim _{N \rightarrow \infty}\left(\hat{\phi}_{1}-\phi_{1}\right) \\
= & -\frac{1+\phi_{1}}{T-1}\left(1-\frac{1-\phi_{1}^{T}}{T\left(1-\phi_{1}\right)}\right)\left[1-\frac{2 \phi_{1}}{\left(1-\phi_{1}\right)(T-1)}\left[1-\frac{1-\phi_{1}^{T}}{T\left(1-\phi_{1}\right)}\right]\right]^{-1}
\end{aligned}
$$

The bias results from the fact that for finite $T$ the averages which appear in the likelihood, and which of course also appear in the resulting estimator, induce correlations among regressors and residuals. These correlations do not vanish as long as $T$ is finite.

Another way of looking at this result is that the LSDV estimator equals the GLS estimator of (2.13) when taking into account the MA structure of the disturbances. Therefore it can be no surprise that the bias of this latter procedure in the AR(1) case with $T=2$ as derived by Chamberlain (1980) equals the bias for the LSDV above in case $T=2$.

As can be seen from the log-likelihoods $l_{F D}^{C}$ and $l^{C}$ or $\tilde{l}^{C}$ we encounter the same problem when considering the $\mathrm{AR}(\mathrm{p})$ model, as the averages $\bar{y}_{i_{1}-j}$ induce correlations amongst regressors and residuals. The conclusion must be that when we condition on the initial observations, MLE does not lead to consistent estimators for the parameters of the AR(p) process in any of the two models. However when $T$ goes to infinity the estimators become consistent, but unfortunately the time dimension in most panel data sets is short.

Comparing the log-likelihoods with the stochastic initial conditions gives us the following important result. The proof is given in the appendix:

Theorem 3 For the log-likelihood functions derived for the model given by (2.4) the following relation holds when we assume the initial observations being stochastic with a marginal distribution given in (2.7)

$$
l_{F D}^{J}-\tilde{l}^{J}=\frac{N}{2} \log (2 \pi)+\frac{N}{2} \log \left(\sigma^{2}\right)-\frac{N}{2} \log \left(\iota_{T+p}^{\prime}\left(V_{(T+p)}^{p}\right)^{-1} \iota_{T+p}\right) .
$$

Here $\iota_{T+p}$ is a $(T+p) \times \mathbb{1}$ vector of ones. 
This theorem has far reaching consequences for the analysis and relations between estimators derived from the likelihoods. It also gives us the opportunity to derive an alternative way of obtaining a consistent estimator for $\phi_{1}, \ldots, \phi_{p}$ as compared to the bias correction method advocated by for instance Kievit (1995).

\subsubsection{Asymptotics and consistency}

In this section we present the asymptotic distributions of the $\mathrm{ML}$ estimators under the various conditions. Some of these results are well-known but we restate them here to give a complete set of results on ML estimation in dynamic panel data models. We will facus on the behavior of the estimators under the different assumptions on $N$ and $T$.

The following result gives us the consistency of the ML estimators based on the likelihoods derived in the previous section when $T \rightarrow \infty$.

Theorem 4 For fuxed $N$ the $M L$ estimators based on $l^{C}, l^{J}, \tilde{l}^{C}, \tilde{l}^{J}$ and $l_{F D}^{C}$ and $l_{F D}^{J}$ are consistent for stationary $A R(p)$ models when $T \rightarrow \infty$ with

$$
\lim _{T \rightarrow \infty} \sqrt{N(T+p)}\left(\left(\begin{array}{c}
\hat{\phi}-\phi \\
\hat{\sigma^{2}}-\sigma^{2}
\end{array}\right)\right) \rightarrow N\left(0,\left[\begin{array}{cc}
\left(V_{p}^{p}\right)^{-1} & 0 \\
0 & 2 \sigma^{4}
\end{array}\right]\right) .
$$

where $\phi=\left(\phi_{1}, \ldots, \phi_{p}\right)^{\prime}$. In addition the ML estimators for $\phi$ based on $l^{C}, \tilde{l}^{C}$ and $l_{F D}^{C}$ are identical.

Proof. The result concerning the asymptotic distribution is well known and can be found in most textbooks on time series. We therefore omit the proof. The result concerning the identical ML estimators follows from the discussion in the previous section.

The crucial assumption in the above theorem is, besides the stationarity, the fact that $T \rightarrow \infty$. As pointed out the estimators will be inconsistent when $T$ is kept finite, except the estimator based on $l_{F D}^{J}$. This result is in fact well-known in time series analysis. We have in fact a system of $N \mathrm{AR}(\mathrm{p})$ models with the same AR coefficients and the same disturbance variance but different intercepts.

On the other hand, by contrast, we have the fact that $l_{F D}^{J}$ is consistent for finite $T$. This is straightforward when we realize that we are dealing with the likelihood of $N$ independent finite dimensional multivariate probability density functions, and standard theory applies. See for instance Amemiya (1985). The variance is given by the inverse of Fisher Information matrix. We have

Theorem 5 The $M L$ estimator based on $l_{F D}^{J}$ is consistent for stationary $A R(p)$ models when $N \rightarrow \infty$. The asymptotic distribution is given by

$$
\lim _{N \rightarrow \infty} \sqrt{N(T+p)}\left(\left(\begin{array}{c}
\hat{\phi}-\phi \\
\hat{\sigma^{2}}-\sigma^{2}
\end{array}\right)\right) \rightarrow N\left(0,\left[I_{F D}^{J}(T)\right]^{-1}\right) .
$$


The Fisher Information matrix is given by

$$
I_{F D}^{J}(T)=\left(\left[\begin{array}{cc}
\frac{T}{T+p}\left(V_{p}^{p}\right) & 0 \\
0 & \frac{1}{2 \sigma^{4}}
\end{array}\right]+\frac{1}{2(T+p)} I C_{p}\right)
$$

where

$$
I C_{p}=\left[\begin{array}{cc}
I C_{\phi \phi^{\prime}} & \frac{1}{\sigma^{2}} \frac{\partial \operatorname{vec}\left(\left(V_{p}^{p}\right)^{-1}\right)^{\prime}}{\partial \phi} \operatorname{vec}\left(V_{p}^{p}\right) \\
\frac{1}{\sigma^{2}} \operatorname{vec}\left(V_{p}^{p}\right)^{\prime} \frac{\left.\partial \operatorname{vec}\left(V_{p}^{p}\right)^{-1}\right)}{\partial \phi^{\prime}} & -\frac{1}{\sigma^{4}}
\end{array}\right]
$$

with

$$
\begin{aligned}
r C_{\phi \phi^{\prime}}= & \left|\left(V_{p}^{p}\right)\right|^{-2} \frac{\partial\left|\left(V_{p}^{p}\right)\right|}{\partial \phi} \frac{\partial\left|\left(V_{p}^{p}\right)\right|}{\partial \phi^{\prime}} \\
& +\frac{\partial v e c\left(\left(V_{p}^{p}\right)^{-1}\right)^{\prime}}{\partial \phi}\left(V_{p}^{p} \otimes V_{p}^{p}\right) \frac{\partial \operatorname{vec}\left(\left(V_{p}^{p}\right)^{-1}\right)}{\partial \phi^{\prime}} \\
& +\frac{\partial^{2} \log \left(c_{T+p}^{\prime}\left(V_{T+p}^{p}\right)^{-1} \iota_{T+p}\right)}{\partial \phi \partial \phi^{\prime}} .
\end{aligned}
$$

In the appendix, Lemma 10 we show that

$$
\frac{\partial^{2} \log \left(\iota_{T+p}^{\prime}\left(V_{T+p}^{p}\right)^{-1} \iota_{T+p}\right)}{\partial \phi \partial \phi^{\prime}}=\frac{\partial^{2} \log \left(\iota_{p}^{\prime}\left(V_{p}^{p}\right)^{-1} \iota_{p}+T\left(1-\phi_{1}-. .-\phi_{p}\right)^{2}\right)}{\partial \phi \partial \phi^{\prime}} .
$$

Observe that the correction term $\frac{1}{2(T+p)} I C_{p}$ for finite $T$ is of order $T$ as expected. For small $T$ this term cannot be neglected. If $T \rightarrow \infty$ the variance converges to the variance of ML estimators as given in Theorem 4.

Proof. Taking into account the remarks already made before the statement of the theorem we only have to calculate the variance of the estimator. The derivation of the Fisher Information is given in the appendix.

Observe that the theorem shows that most terms of 'correction' $I C_{p}$, result as a correction for the initial observations. When also $T \rightarrow \infty$ the influence of the initial conditions diminishes. The term (2.33) however stems from the first difference transformation, but the information loss implied by this transformation also diminishes when $T \rightarrow \infty$. Therefore it is not surprising that we obtain

$$
\lim _{T \rightarrow \infty} \lim _{N \rightarrow \infty} \sqrt{N T}\left(\left(\begin{array}{c}
\hat{\phi}-\phi \\
\hat{\sigma^{2}}-\sigma^{2}
\end{array}\right)\right) \rightarrow N\left(0,\left[\begin{array}{cc}
\left(V_{p}^{p}\right)^{-1} & 0 \\
0 & 2 \sigma^{4}
\end{array}\right]\right)
$$

the same distribution as given in Theorem 4. Further we note that although the expressions appear complicated we only have to deal with symmetric matrices of order $p \times p$. To obtain explicit expressions for $\left(V_{p}^{p}\right)^{-1}$ we refer the reader to Galbraith and Galbraith (1974) or section 5 of this chapter where we give a summary of their results. 


\subsection{On the asymptotic bias of the MLE in the non- transformed model}

In the previous section we have derived the asymptotic distributions of the ML estimators based on the stochastic initial conditions for the two models under different assumptions on $N$. Now the question arises which estimator is most appropriate to use under the different conditions for $N$ and $T$. As the ML estimator in the non-transformed model is inconsistent when $T$ is fixed we start by deriving its bias based on the likelihood $\vec{l}$. This enables us later to calculate the expected mean square error of the $\mathrm{ML}$ estimator in the non-transformed model. The theorem can also be used to correct the ML estimator and make it consistent. The resulting estimator might be an alternative to the bias correction method of Kievit (1995).

Theorem 6 The asymptotic bias of the ML estimator based on the non-transformed model with stochastic initial observations, with log-bikelihood $\tilde{l}^{u}$ is in first order approximated by

$$
\begin{aligned}
\lim _{N \rightarrow \infty}\left(\begin{array}{c}
\hat{\phi_{N}}-\phi \\
\sigma_{N}^{2}-\sigma^{2}
\end{array}\right)= & -\frac{1}{2(T+p)}\left[\begin{array}{cc}
I_{\phi \phi \phi^{\prime}} & I_{\sigma^{2} \phi^{\prime}} \\
I_{\phi \sigma^{2}} & I_{\sigma^{2} \sigma^{2}}
\end{array}\right]^{-1}\left(\begin{array}{c}
\xi_{T} \\
\frac{1}{\sigma^{2}}
\end{array}\right) \\
& +o_{p}\left(\frac{1}{2(T+p)}\right)
\end{aligned}
$$

where

$$
\begin{aligned}
I_{\phi \phi}= & \frac{T}{T+p} V_{p}^{p}+\frac{1}{2(T+p)}\left|\left(V_{p}^{p}\right)\right|^{-2} \frac{\partial\left|\left(V_{p}^{p}\right)\right|^{\prime}}{\partial \phi} \frac{\partial\left|\left(V_{p}^{p}\right)\right|}{\partial \phi^{\prime}} \\
& +\frac{1}{2(T+p)} \frac{\partial \operatorname{vec}\left(\left(V_{p}^{p}\right)^{-1}\right)^{\prime}}{\partial \phi}\left(V_{p}^{p} \otimes V_{p}^{p}\right) \frac{\partial \operatorname{vec}\left(\left(V_{p}^{p}\right)^{-1}\right)}{\partial \phi^{\prime}}, \\
I_{\sigma^{2} \sigma^{2}}= & \frac{1}{2 \sigma^{4}} \\
I_{\sigma^{2} \phi^{\prime}}= & \frac{1}{2 \sigma^{2}(T+p)} \operatorname{vec}\left(V_{p}^{p}\right)^{\prime} \frac{\partial \operatorname{vec}\left(\left(V_{p}^{p}\right)^{-1}\right)}{\partial \phi^{\prime}}
\end{aligned}
$$

and

$$
\xi_{T}=-\frac{\frac{\partial t_{T+p}^{\prime}\left(V_{T+p}^{p}\right)^{-1} \iota_{T+p}}{\partial \phi}}{\iota_{T+p}^{\prime}\left(V_{T+p}^{p}\right)^{-1} \iota_{T+p}} .
$$

The matrix in the bias equals the inverse of the information matrix of the MLE based on the direct model. Observe further that the bias for $\phi$ is of magnitude $O_{p}\left(T^{-1}\right)$. This can easily be checked from the fact that the leading term in $I_{\phi \phi}$ is $V_{p}^{p}$ which does not depend on $T$ and Corollary 9.5 in the appendix which states that the leading term of $\xi_{T}$ is a constant. We now state the proof.

Proof. To ease notation we introduce

$$
f_{N}(x)=\frac{1}{N(T+p)} l_{F D}^{J}, \quad g_{N}(x)=\frac{1}{N(T+p)} \stackrel{\sim}{l}^{J}
$$


and

$$
h(x)=\frac{1}{2(T+p)}\left(\log (2 \pi)+\log \left(\sigma^{2}\right)-\log \left(\iota_{T+F}^{\prime}\left(V_{T+p}^{p}\right)^{-1} \iota_{T+p}\right)\right) .
$$

where $x=\left(\phi^{\prime}, \sigma^{2}\right)^{\prime}$. Further we introduce $f(x)$ and $g(x)$ as their limits when $N \rightarrow \infty$. Observe that these limits exist and that we have $f_{N}(x) \rightarrow f(x)$ and $g_{N}(x) \rightarrow g(x)$ a.s. uniformly in $T$. Then using (4.7) we obtain

$$
\frac{1}{N}\left(l_{F D}^{J}(x)-\tilde{l}^{J}(x)\right)=f_{N}(x)-g_{N}(x)=h(x) .
$$

Suppose that $x_{0}=\arg \min \left(f_{N}(x)\right)$ and $x_{1}=\arg \min \left(g_{N}(x)\right)$. Observe that by Theorem $5 x_{0}$ is a consistent estimator. Then we have first order conditions $f_{N}^{\prime}\left(x_{0}\right)=g_{N}^{\prime}\left(x_{1}\right)=0$, where $f^{\prime}$ indicates differentiation with respect to $x$. Write $x_{1}=x_{0}+b$, where $b$ has the same dimension as $x$, we then have $f_{N}^{\prime}\left(x_{0}+b\right)=h^{\prime}\left(x_{0}+b\right)$. By the fact that $f_{N}$ and $g_{N}$ are twice continuously differentiable in $x_{0}$, we have $f_{N}^{\prime}\left(x_{0}+b\right)=f_{N}^{\prime \prime}\left(x_{0}\right) b+R_{N}$ and $h^{\prime}\left(x_{0}+b\right)=h^{\prime}\left(x_{0}\right)+h^{\prime \prime}\left(x_{0}\right) b+\bar{R}$, where

$$
\lim _{|b| \rightarrow 0} \frac{\left|R_{N}\right|}{|b|}=0 \text { and } \lim _{|b| \rightarrow 0} \frac{|\bar{R}|}{|b|}=0
$$

Now we can take the limit for $N \rightarrow \infty$, and the result still holds. Equating these two expressions gives, provided $\left[f^{\prime \prime}\left(x_{0}\right)-h^{\prime \prime}\left(x_{0}\right)\right]$ is non-singular,

$$
b=\left[f^{\prime \prime}\left(x_{0}\right)-h^{\prime \prime}\left(x_{0}\right)\right]^{-1} h^{\prime}\left(x_{0}\right)+R_{N}^{*}
$$

with $\lim _{|b| \rightarrow 0} \frac{\left|R_{s}^{*}\right|}{|b|}=0$. Now observe that $-f^{\prime \prime}\left(x_{0}\right)$ is the information matrix of the MLE based on the first difference. Therefore it follows that $\left[f^{\prime \prime}\left(x_{0}\right)-h^{\prime \prime}\left(x_{0}\right)\right]$ equals minus the information matrix of the MLE for the non-transformed model (with log-likelihood function $l^{J}$ ):

$$
b \cong-\left[I^{J}\left(x_{0}\right)\right]^{-1} h^{\prime}\left(x_{0}\right) .
$$

Note that $\left[I^{J}\right]^{-1}$ is not the variance of the MLE because the MLE is inconsistent. In the appendix Lemma 9.6 we calculated $I^{J}$. Finally deriving $h^{\prime}$ gives the stated result.

Observe that we can also employ the relation $-g_{N}^{\prime}\left(x_{1}-b\right)=h_{N}^{\prime}\left(x_{1}-b\right)$ in the above proof. Doing so leads, using similar arguments as in the proof, to

$$
b=\left[g^{\prime \prime}\left(x_{1}\right)+h^{\prime \prime}\left(x_{1}\right)\right]^{-1} h^{\prime}\left(x_{1}\right)=-\left[I_{F D}^{J}\left(x_{1}\right)\right]^{-1} h^{\prime}\left(x_{1}\right)
$$

where $-\left[I_{F D}^{J}\left(x_{1}\right)\right]^{-1}$ is minus the inverse of the information of the MLE based on the differenced model calculated at the -biased- estimate of the MLE based on the nontransformed model. Note that we might use this result to correct the MLE based on the non-transformed model.

Observe also that the technique we used in the proof to obtain the bias of the MLE in the non-transformed model also works in general. Assume that one has two estimators of a parameter which result from optimizing two different objective functions, like a likelihood or a quadratic distance function. Assuming that it is known that one of the resulting 

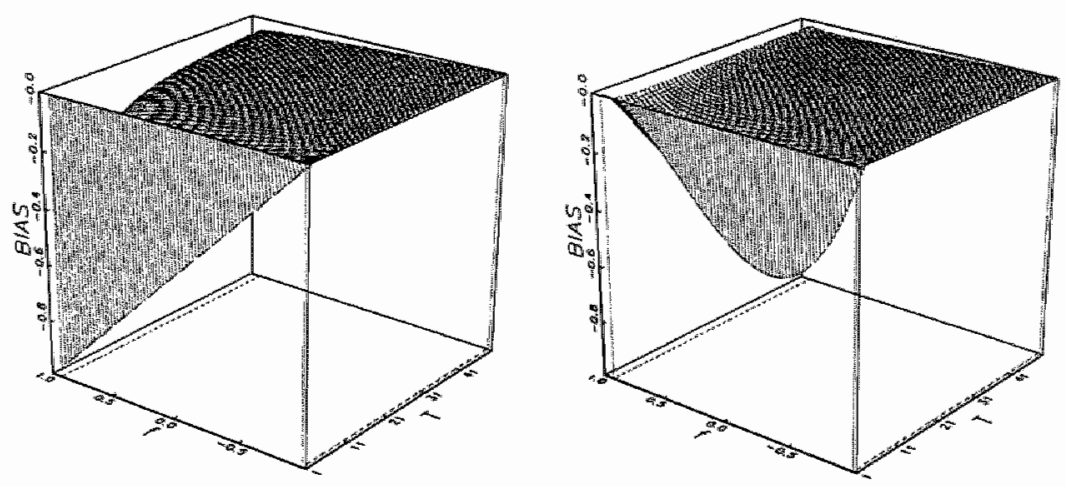

Figure 2.1: Biases in the AR(1) model. 
estimators is consistent and unbiased then one can construct the bias of the other estimator by analyzing the difference between the two objective functions, as is done in the proof.

An interesting question concerning the AR(1) model is in how the bias of exact MLE as given above compares with the bias of the LSDV as given in (2.26). As the only difference between the two estimators is the treatment of the initial observations we expect that for larger $T$, i.e. when the influence of the initial observations diminishes, they should be close to each other. To compare the two asymptotic biases we consider the following graph which plots the bias of the LSDV and the exact MLE. Three observations can be made from Figure 2.1. Firstly the bias of the exact MLE is less severe for small $T$ than that of the LSDV estimator. Secondly for large $T$ the biases converge to zero at the same rate and are comparable in size. Thirdly the bias of the LSDV is much more asymmetric than the one of the exact MLE.

As for larger $T$ the LSDV and exact MLE have a comparable bias. The bias for the latter, given by Theorem 6, might be used to approximate the bias of the LSDV in higher order $\mathrm{AR}(\mathrm{p})$ processes.

\subsection{Calculation of the estimator}

Although the estimation can be done with any numerical procedure available we present here some techniques we found useful in practice. To estimate the parameters $\phi$ and $\sigma^{2}$ we have to maximize the $\log$-likelihood

$$
\begin{aligned}
l_{F D}^{J}= & \tilde{l}^{J}+\frac{N}{2} \log \left(\sigma^{2}\right)-\frac{N}{2} \log \left(\iota_{T+p}^{\prime}\left(V_{T+p}^{p}\right)^{-1} \iota_{T+p}\right) \\
\propto & -\frac{N(T+p-1)}{2} \log \left(\sigma^{2}\right)-\frac{N}{2} \log \left(\left|V_{T+p}^{p}\right| l_{T+p}^{\prime}\left(V_{T+p}^{p}\right)^{-1} \iota_{T+p}\right) \\
& -\frac{1}{2 \sigma^{2}} \sum_{i=1}^{N} y_{i}^{\prime} D_{T+p}^{\prime}\left[\left(D_{T+p} V_{T+p}^{p} D_{T+p}^{\prime}\right)^{-1}\right] D_{T+p} y_{i} .
\end{aligned}
$$

To obtain analytical results is possible but very tedious and hardly manageable when the order of the process becomes large. However the first order condition for $\sigma^{2}$ is simple and gives as solution

$$
\sigma^{2}=\frac{1}{N(T+p-1)} \sum_{i=1}^{N} y_{i}^{\prime} D_{T+p}^{t}\left[\left(D_{T+p} V_{T+p}^{p} D_{T+p}^{x}\right)^{-1}\right] D_{T+p} y_{i} .
$$

Concentrating (2.44) with respect to $\sigma^{2}$ gives

$$
\begin{aligned}
l_{F D}^{J}= & -(T+p-1) \log \left(\frac{1}{N(T+p-1)} \sum_{i=1}^{N} y_{i}^{\prime} D_{T+p}^{\prime}\left[\left(D_{T+p} V_{T+p}^{p} D_{T+p}^{\prime}\right)^{-1}\right] D_{T+p} y_{i}\right. \\
& -\log \left(\left|V_{(T+p)}^{p p}\right| \iota_{T+p}^{\prime}\left(V_{T+p}^{p}\right)^{-1} \iota_{T+p}\right) .
\end{aligned}
$$

We now observe that

$$
y_{i}^{\prime} D_{T+p}^{\prime}\left[\left(D_{T+p} V_{T+p}^{p} D_{T+p}^{\prime}\right)^{-1}\right] D_{T+p} y_{i}=y_{i}^{\prime}\left(V_{T+p}^{p}\right)^{-1} y_{i}-\frac{\left(t_{T+p}^{\prime}\left(V_{T+p}^{p}\right)^{-1} y_{i}\right)^{2}}{t_{T+p}^{\prime}\left(V_{T+p}^{p}\right)^{-1}{ }_{T+p}}
$$


which can be numerically easily calculated. We have to optimize (2.46) with respect to Q. This can be done numerically. Observe that for this we need $\left(V_{T+p}^{p}\right)^{-1}$ which we can calculate using the results of Galbraith and Galbraith (1974).

For the $\left(V_{T}^{p}\right)^{-1}=\left[\left(V_{p}^{p}\right)^{-1}\right]_{r=1, s=1}^{T T}$ we have

$$
\left[\left(V_{T}^{p}\right)^{-1}\right]_{r s}=\sum_{j=0}^{c} \phi_{j} \phi_{j+s-r}-\sum_{j=d}^{p+r-s} \phi_{j} \phi_{j+s-r,}, \quad 1 \leq r \leq s \leq T
$$

where

$$
c=\min \{r-1, p-r-s, T-s\}, \quad d=\max \{r-1, T-s\}
$$

and $\phi_{0}=-1$. The sums equal zero if its upper limit is less than its lower limit. Observe that the second sum is zero unless $T-p+1 \leq r<s \leq p$, and both sums equal zero if $s-r>p$. For $\left(V_{p}^{p}\right)^{-1}=\left[\left(V_{p}^{p}\right)^{-1}\right]_{r=1, s=1}^{p p}$ this simplifies to

$$
\left[\left(V_{p}^{p}\right)^{-1}\right]_{r s}=\sum_{j=0}^{r-1} \phi_{j} \phi_{j+s-r}-\sum_{j=p+1-s}^{p+r-s} \phi_{j} \phi_{j+s-r}, \quad 1 \leq r \leq s \leq p .
$$

When $T \geq 2 p$, which is the case in most situations we are interested in, there is however a more convenient method. Form the matrix

$$
A_{T+p}^{p *}=-\left(\begin{array}{lllllllllll}
-1 & 0 & & & & & & & & 0 \\
\phi_{1} & -1 & & & & & & & & \\
\phi_{2} & \phi_{1} & -1 & & & & & & & \\
. & . . & . . & . . & & & & & & \\
\phi_{p} & . . & \phi_{2} & \phi_{1} & -1 & & & & & \\
0 & \phi_{p} & \ldots & \phi_{2} & \phi_{1} & -1 & & . & & \\
& 0 & \phi_{p} & \ldots & \phi_{2} & \phi_{1} & -1 & & & \\
& & & . & . & . & . & -1 & & . \\
& & & & . & . & . & . & . & 0 \\
0 & & & & 0 & \phi_{p} & & \phi_{2} & \phi_{1} & -1
\end{array}\right)
$$

calculate $A_{T}^{p * s} A_{T}^{p *}$ then use the property that the matrix $\left(V_{T+p}^{p}\right)^{-1}$ is symmetric in both principal axes to replace the left upper comer $p \times p$ matrix with the right elements from the right lower corner $p \times p$ matrix, and we have obtained $\left(V_{T+p}^{p}\right)^{-1}$. When using computer packages like Gauss this is no problem.

Now we have a convenient way of obtaining $\left(V_{T+p}^{p}\right)^{-1}$ without having to calculate its inverse. We can optimize (2.44) using any numerical optimization procedure. As starting values we can use the LSDV estimate.

To conduct inference one can use Theorem 5 with the estimated $\hat{\phi}$ substituted in the variance formulas. In the next two sections we investigate how these compare with the theoretical asymptotic values in some specific cases. 


\subsection{Examples 1: the AR(1) model.}

The AR(1) model has been studied extensively in the past. Therefore the results derived in the previous section are first illustrated by this example. In this case the distribution for the initial observation is given by $y_{i, 0} \sim N\left(\mu_{i}, \frac{\sigma^{2}}{1-\phi_{1}^{2}}\right)$ with $\mu_{i}=\left(1-\phi_{1}\right)^{-1} c_{i}$. To calculate the variance of the MLE based on the first differenced model and the bias of the MLE based on the non-transformed model we need the following results. We have

$$
V_{1}^{1}=\frac{1}{1-\phi_{1}^{2}}, \quad \frac{\partial\left(V_{1}^{1}\right)^{-1}}{\partial \phi_{1}}=1-2 \phi_{1} .
$$

For the relevant determinant we find $\left|V_{1}^{1}\right|=\frac{1}{1-\phi_{1}^{2}}$ and further $\iota^{\prime}\left(V_{1}^{1}\right)^{-1} \iota=\left(1-\phi_{1}^{2}\right)$. Note that the relation given by Theorem 4.7 equals

$$
l_{F D}^{J}-\tilde{L}^{J}=\frac{N}{2} \log (2 \pi)+\frac{N}{2} \log \left(\sigma^{2}\right)-\frac{N}{2} \log \left(\left(1-\phi_{1}^{2}\right)+(T-1)\left(1-\phi_{1}\right)^{2}\right)
$$

in estimation however we do not need it. The variance matrix, the inverse of $I_{F D}^{J}(T)$, as given in Theorem 5 where

$$
\begin{aligned}
I_{F D}^{J}(T)= & {\left[\begin{array}{ll}
\frac{1}{1-\phi_{1}^{2}} & 0 \\
0 & \frac{1}{2 \sigma^{2}}
\end{array}\right]+\frac{1}{2 T+2} } \\
& \times\left[\begin{array}{cc}
4 \phi_{1}^{2}+\left(\frac{-\phi_{1}}{1+\phi_{1}}\right)^{2}-f\left(\phi_{1}\right) & {\left[\begin{array}{c}
\frac{1}{\sigma^{2}} \frac{-\phi_{1}}{1+\phi_{1}} \\
\frac{1}{\sigma^{2}} \frac{-\phi_{1}}{1+\phi_{1}}
\end{array}\right.} \\
-\frac{1}{\sigma^{4}}
\end{array}\right],
\end{aligned}
$$

where $f\left(\phi_{1}\right)=2 \frac{4 \phi_{1}^{2}-2 T+6 T \phi_{1}-4 T \phi_{1}^{2}-4 \phi_{1}+T^{2}-2 T^{2} \phi_{1}+T^{2} \phi_{1}^{2}+2}{\left(-2 \phi_{1}^{2}+T-2 T \phi_{1}+T \phi_{1}^{2}+2 \phi_{1}\right)^{2}}$. For the bias of the MLE in the transformed model we have

$$
\begin{aligned}
B(T)= & -\frac{1}{2 T}\left[\begin{array}{cc}
\frac{1}{1-\phi_{1}^{2}}+\frac{1}{2 T}\left[4 \phi_{1}^{2}+\left(\frac{-\phi_{1}}{1+\phi_{1}}\right)^{2}\right] & \frac{1}{2 T \sigma^{2}} \frac{-\phi_{1}}{1+\phi_{1}} \\
\frac{1}{2 T \sigma^{2}} \frac{-\phi_{1}}{1+\phi_{1}} & \frac{1}{2 \sigma^{4}}
\end{array}\right]^{-1} \\
& \times\left[\begin{array}{c}
2 \frac{-2 \phi_{1}-T+T \phi_{1}+1}{-2 \phi_{1}^{2}+T-2 T \phi_{1}+T \phi_{1}^{2}+2 \phi_{1}} \\
\frac{1}{\sigma^{4}}
\end{array}\right] .
\end{aligned}
$$

To see in how far these asymptotic values hold in practice we now conduct a small sample size simulation where we calculate the MLE based on the first differenced model and the MLE based on the direct model.

We have simulated for the different sample sizes of combinations of $N$ and $T 100$ data sets with DGP

$$
y_{i, t}=\phi_{1} y_{i, t-1}+c_{i}+\varepsilon_{i, t},
$$

with $E\left(\varepsilon_{i, t}\right)^{2}=\sigma^{2}=1$ and the $c_{i}$ independently drawn from $N(1,3)$, the initial values independently drawn form $y_{i, 0}=N\left(c_{i} /\left(1-\phi_{1}\right), 1 /\left(1-\phi_{1}^{2}\right)\right)$ for two different values of $\phi_{1 .} \phi_{1}=-0.6$ and $\phi_{1}=0.6$. The results are given in the Tables 2.1 to 2.4 . 


\begin{tabular}{|cccccc|}
\hline $\mathbf{T}$ & $\mathbf{N}$ & avebias & asybias & stdv & asystdv \\
\hline 10 & 10 & -0.0496 & -0.0333 & 0.0729 & 0.0763 \\
10 & 25 & -0.0715 & -0.0333 & 0.0384 & 0.0482 \\
10 & 50 & -0.0695 & -0.0333 & 0.0295 & 0.0341 \\
10 & 75 & -0.0644 & -0.0333 & 0.0268 & 0.0279 \\
25 & 10 & -0.0196 & -0.0145 & 0.0446 & 0.0496 \\
25 & 25 & -0.0294 & -0.0145 & 0.0303 & 0.0314 \\
25 & 50 & -0.0269 & -0.0145 & 0.0218 & 0.0222 \\
25 & 75 & -0.0286 & -0.0145 & 0.0180 & 0.0181 \\
50 & 10 & -0.0138 & -0.0076 & 0.0356 & 0.0354 \\
50 & 25 & -0.0155 & -0.0076 & 0.0199 & 0.0224 \\
50 & 50 & -0.0142 & -0.0076 & 0.0171 & 0.0158 \\
50 & 75 & -0.0141 & -0.0076 & 0.0142 & 0.0129 \\
75 & 10 & -0.0085 & -0.0051 & 0.0291 & 0.0290 \\
75 & 25 & -0.0078 & -0.0051 & 0.0157 & 0.0184 \\
75 & 50 & -0.0078 & -0.0051 & 0.0129 & 0.0130 \\
75 & 75 & -0.0090 & -0.0051 & 0.0089 & 0.0106 \\
\hline Value autoregressive parameter: $\phi_{1}=-0.6$. \\
\hline
\end{tabular}

Table 2.1: Results for the MLE in the nontransformed model, case 1.

\begin{tabular}{|cccccc|}
\hline T & $\mathbf{N}$ & avebias & asybias & stdv & asystdv \\
\hline 10 & 10 & -0.0088 & 0.0000 & 0.0817 & 0.0668 \\
10 & 25 & -0.0328 & 0.0000 & 0.0431 & 0.0423 \\
10 & 50 & -0.0306 & 0.0000 & 0.0327 & 0.0299 \\
10 & 75 & -0.0246 & 0.0000 & 0.0299 & 0.0244 \\
25 & 10 & -0.0033 & 0.0000 & 0.0465 & 0.0466 \\
25 & 25 & -0.0134 & 0.0000 & 0.0316 & 0.0294 \\
25 & 50 & -0.0108 & 0.0000 & 0.0228 & 0.0208 \\
25 & 75 & -0.0127 & 0.0000 & 0.0187 & 0.0170 \\
50 & 10 & -0.0058 & 0.0000 & 0.0363 & 0.0342 \\
50 & 25 & -0.0075 & 0.0000 & 0.0203 & 0.0217 \\
50 & 50 & -0.0062 & 0.0000 & 0.0175 & 0.0153 \\
50 & 75 & -0.0062 & 0.0000 & 0.0144 & 0.0125 \\
75 & 10 & -0.0032 & 0.0000 & 0.0295 & 0.0284 \\
75 & 25 & -0.0024 & 0.0000 & 0.0159 & 0.0179 \\
75 & 50 & -0.0025 & 0.0000 & 0.0131 & 0.0127 \\
75 & 75 & -0.0037 & 0.0000 & 0.0090 & 0.0104 \\
\hline \multicolumn{5}{|c|}{ Value autoregressive parameter: $\phi_{1}=-0.6}$. \\
\hline
\end{tabular}

Table 2.2: Results for the MLE in the first differenced model, case 1. 


\begin{tabular}{|cccccc|}
\hline $\mathrm{T}$ & $\mathrm{N}$ & avebias & asybias & stdv & asystdv \\
\hline 10 & 10 & -0.1698 & -0.1011 & 0.0939 & 0.0763 \\
10 & 25 & -0.1521 & -0.1011 & 0.0608 & 0.0482 \\
10 & 50 & -0.1354 & -0.1011 & 0.0444 & 0.0341 \\
10 & 75 & -0.1446 & -0.1011 & 0.0362 & 0.0279 \\
25 & 10 & -0.0522 & -0.0528 & 0.0488 & 0.0496 \\
25 & 25 & -0.0490 & -0.0528 & 0.0359 & 0.0314 \\
25 & 50 & -0.0534 & -0.0528 & 0.0255 & 0.0222 \\
25 & 75 & -0.0522 & -0.0528 & 0.0201 & 0.0181 \\
50 & 10 & -0.0312 & -0.0290 & 0.0416 & 0.0354 \\
50 & 25 & -0.0234 & -0.0290 & 0.0265 & 0.0224 \\
50 & 50 & -0.0239 & -0.0290 & 0.0167 & 0.0158 \\
50 & 75 & -0.0242 & -0.0290 & 0.0114 & 0.0129 \\
75 & 10 & -0.0197 & -0.0200 & 0.0291 & 0.0290 \\
75 & 25 & -0.0160 & -0.0200 & 0.0197 & 0.0184 \\
75 & 50 & -0.0178 & -0.0200 & 0.0139 & 0.0130 \\
75 & 75 & -0.0178 & -0.0200 & 0.0102 & 0.0106 \\
\hline \multicolumn{5}{c}{ Value autoregressive parameter: $\phi_{1}=0.6}$. \\
\hline
\end{tabular}

Table 2.3: Results for the MLE in nontransformed model, case 2.

\begin{tabular}{|cccccc|}
\hline T & $\mathbf{N}$ & avebias & asybias & stdv & asystdv \\
\hline 10 & 10 & 0.0074 & 0.0000 & 0.1112 & 0.0818 \\
10 & 25 & 0.0298 & 0.0000 & 0.0699 & 0.0517 \\
10 & 50 & 0.0483 & 0.0000 & 0.0524 & 0.0366 \\
10 & 75 & 0.0367 & 0.0000 & 0.0430 & 0.0299 \\
25 & 10 & 0.0150 & 0.0000 & 0.0515 & 0.0523 \\
25 & 25 & 0.0187 & 0.0000 & 0.0375 & 0.0330 \\
25 & 50 & 0.0139 & 0.0000 & 0.0270 & 0.0234 \\
25 & 75 & 0.0153 & 0.0000 & 0.0212 & 0.0191 \\
50 & 10 & 0.0015 & 0.0000 & 0.0424 & 0.0366 \\
50 & 25 & 0.0094 & 0.0000 & 0.0271 & 0.0231 \\
50 & 50 & 0.0089 & 0.0000 & 0.0171 & 0.0164 \\
50 & 75 & 0.0086 & 0.0000 & 0.0117 & 0.0134 \\
75 & 10 & 0.0019 & 0.0000 & 0.0295 & 0.0297 \\
75 & 25 & 0.0056 & 0.0000 & 0.0200 & 0.0188 \\
75 & 50 & 0.0039 & 0.0000 & 0.0142 & 0.0133 \\
75 & 75 & 0.0038 & 0.0000 & 0.0103 & 0.0108 \\
\hline \multicolumn{5}{|c}{ Value autoregressive parameter: $\phi_{1}=0.6}$. \\
\hline \multicolumn{7}{c}{}
\end{tabular}

Table 2.4: Results for the MLE in the first differenced model, case 2. 
The columns "asybias" and "asystdv" denote the theoretical asymptotic bias and standard deviation of the estimator as derived in Theorems 3,4 and 5. The "avebias" and "stdv" are the average bias and standard deviation of the estimator based on the 100 replications. In Table 2.1 we see that the bias is substantial across the whole range of combinations of $N$ and $T$ even when $T=75$.

In Table 2.2 we see that although the MLE based on first differences has asymptotically no bias it is clear that in our simulation there is a small negative finite sample bias. It is however much smaller than the bias of the direct MLE. It is also much smaller than the standard error of the estimator. This is in contrast to the results in Table 2.1 for the MLE based on the direct model. When one compares Table 2.1 with Table 2.3 there is a clear asymmetry in the bias of the MLE based on the direct model. In Table 2.4 we see that the finite sample bias we found in the MLE based on the first differenced model again appears but now with opposite sign. From the simulations it becomes clear that the theoretical asymptotic bias for the AR(1) case with negative coefficient underestimates the bias appearing in practice for small $T$.

\subsection{Examples 2: the AR(2) model.}

To investigate how the derived results hold in higher order AR models we start with the $A R(2)$ model. The matrices that are relevant for the derivation of the bias and variances are now given by

$$
\begin{aligned}
V_{2}^{2} & =\frac{1}{\left(1+\phi_{2}\right)^{2}\left[\left(1-\phi_{2}\right)^{2}-\phi_{1}^{2}\right]}\left[\begin{array}{cc}
1-\phi_{2}^{2} & \phi_{1}+\phi_{1} \phi_{2} \\
\phi_{1}+\phi_{1} \phi_{2} & 1-\phi_{2}^{2}
\end{array}\right], \\
\left(V_{2}^{2}\right)^{-1} & =\left[\begin{array}{cc}
1-\phi_{2}^{2} & -\left(\phi_{1}+\phi_{1} \phi_{2}\right) \\
-\left(\phi_{1}+\phi_{1} \phi_{2}\right) & 1-\phi_{2}^{2}
\end{array}\right]
\end{aligned}
$$

and

$$
\frac{\partial \operatorname{vec}\left(V_{2}^{2}\right)^{-1}}{\partial \phi^{\prime}}=\left[\begin{array}{ll}
0 & -2 \phi_{2} \\
-1-\phi_{2} & -\phi_{1} \\
-1-\phi_{2} & -\phi_{1} \\
0 & -2 \phi_{2}
\end{array}\right] .
$$

For the determinant we find

$$
\left|V_{2}^{2}\right|=\frac{1}{\left(1+\phi_{2}\right)^{2}\left[\left(1-\phi_{2}\right)^{2}-\phi_{1}^{2}\right]}
$$

and $\iota_{2}^{\prime}\left(V_{2}^{2}\right)^{-1} \iota_{2}=2\left(1-\phi_{2}^{2}\right)-2\left(\phi_{1}+\phi_{1} \phi_{2}\right)$. Finally the relation given by Theorem 3 equals

$$
l_{F D}^{S}-\tilde{l}_{D}^{S} \propto \frac{N}{2} \log \left(\sigma^{2}\right)+\frac{N}{2} \log \left(2\left(1-\phi_{2}^{2}\right)-2\left(\phi_{1}+\phi_{1} \phi_{2}\right)+(T-2)\left(1-\phi_{1}-\phi_{2}\right)^{2}\right) .
$$

Again we conducted a small sample size simulation. We again created for every combination of $T$ and $N 100$ samples of the following DGP:

$$
y_{i, t}=\phi_{1} y_{i, t-1}+\phi_{2} y_{i, t-2}+c_{i}+\varepsilon_{i, t}
$$

with 


\begin{tabular}{|cccccccccc|}
\hline $\mathrm{T}$ & $\mathrm{N}$ & avebias & avebias & asybias & asybias & stdv & stdv & asystdv & asystdv \\
\hline & & $\phi_{1}$ & $\phi_{2}$ & $\phi_{1}$ & $\phi_{2}$ & $\phi_{1}$ & $\phi_{2}$ & $\phi_{1}$ & $\phi_{2}$ \\
\hline 10 & 10 & -0.0495 & -0.0547 & -0.0383 & -0.0237 & 0.1001 & 0.0817 & 0.0837 & 0.0837 \\
10 & 25 & -0.0416 & -0.0760 & -0.0383 & -0.0237 & 0.0588 & 0.0486 & 0.0529 & 0.0529 \\
10 & 50 & -0.0435 & -0.0766 & -0.0383 & -0.0237 & 0.0455 & 0.0368 & 0.0374 & 0.0374 \\
10 & 75 & -0.0403 & -0.0706 & -0.0383 & -0.0237 & 0.0364 & 0.0302 & 0.0306 & 0.0306 \\
25 & 10 & -0.0209 & -0.0378 & -0.0154 & -0.0127 & 0.0565 & 0.0591 & 0.0558 & 0.0558 \\
25 & 25 & -0.0052 & -0.0277 & -0.0154 & -0.0127 & 0.0637 & 0.0725 & 0.0353 & 0.0353 \\
25 & 50 & -0.0181 & -0.0286 & -0.0154 & -0.0127 & 0.0256 & 0.0243 & 0.0249 & 0.0249 \\
25 & 75 & -0.0199 & -0.0286 & -0.0154 & -0.0127 & 0.0218 & 0.0168 & 0.0204 & 0.0204 \\
50 & 10 & -0.0074 & -0.0214 & -0.0077 & -0.0070 & 0.0443 & 0.0430 & 0.0402 & 0.0402 \\
50 & 25 & -0.0068 & -0.0166 & -0.0077 & -0.0070 & 0.0262 & 0.0261 & 0.0254 & 0.0254 \\
50 & 50 & -0.0069 & -0.0155 & -0.0077 & -0.0070 & 0.0163 & 0.0177 & 0.0180 & 0.0180 \\
50 & 75 & -0.0076 & -0.0145 & -0.0077 & -0.0070 & 0.0149 & 0.0155 & 0.0147 & 0.0147 \\
75 & 10 & -0.0025 & -0.0103 & -0.0051 & -0.0048 & 0.0339 & 0.0327 & 0.0330 & 0.0330 \\
75 & 25 & -0.0046 & -0.0089 & -0.0051 & -0.0048 & 0.0232 & 0.0213 & 0.0209 & 0.0209 \\
75 & 50 & -0.0056 & -0.0084 & -0.0051 & -0.0048 & 0.0152 & 0.0148 & 0.0148 & 0.0148 \\
75 & 75 & -0.0050 & -0.0091 & -0.0051 & -0.0048 & 0.0123 & 0.0121 & 0.0121 & 0.0121 \\
\hline Values autoregressive parameters: $\phi_{1}=0.3$ and & $\phi_{2}=-0.4$. & & \\
\hline
\end{tabular}

Table 2.5: Results for the MLE in nontransformed model, ar(2).

\begin{tabular}{|cccccccccc|}
\hline $\mathrm{T}$ & $\mathrm{N}$ & avebias & avebias & asybias & asybias & stdv & stdv & asystdv & asystdv \\
\hline & & $\phi_{1}$ & $\phi_{2}$ & $\phi_{1}$ & $\phi_{2}$ & $\phi_{1}$ & $\phi_{2}$ & $\phi_{1}$ & $\phi_{2}$ \\
\hline 10 & 10 & 0.0181 & 0.0129 & 0.0000 & 0.0000 & 0.0993 & 0.0923 & 0.0883 & 0.0822 \\
10 & 25 & 0.0235 & -0.0113 & 0.0000 & 0.0000 & 0.0582 & 0.0548 & 0.0558 & 0.0520 \\
10 & 50 & 0.0208 & -0.0130 & 0.0000 & 0.0000 & 0.0459 & 0.0414 & 0.0395 & 0.0367 \\
10 & 75 & 0.0246 & -0.0060 & 0.0000 & 0.0000 & 0.0353 & 0.0341 & 0.0322 & 0.0300 \\
25 & 10 & 0.0036 & -0.0135 & 0.0000 & 0.0000 & 0.0565 & 0.0614 & 0.0569 & 0.0554 \\
25 & 25 & 0.0132 & -0.0097 & 0.0000 & 0.0000 & 0.0350 & 0.0326 & 0.0360 & 0.0350 \\
25 & 50 & 0.0065 & -0.0040 & 0.0000 & 0.0000 & 0.0254 & 0.0253 & 0.0254 & 0.0248 \\
25 & 75 & 0.0047 & -0.0040 & 0.0000 & 0.0000 & 0.0218 & 0.0175 & 0.0208 & 0.0202 \\
50 & 10 & 0.0046 & -0.0094 & 0.0000 & 0.0000 & 0.0442 & 0.0439 & 0.0406 & 0.0401 \\
50 & 25 & 0.0054 & -0.0045 & 0.0000 & 0.0000 & 0.0260 & 0.0267 & 0.0257 & 0.0253 \\
50 & 50 & 0.0053 & -0.0034 & 0.0000 & 0.0000 & 0.0163 & 0.0181 & 0.0181 & 0.0179 \\
50 & 75 & 0.0045 & -0.0023 & 0.0000 & 0.0000 & 0.0148 & 0.0158 & 0.0148 & 0.0146 \\
75 & 10 & 0.0056 & -0.0022 & 0.0000 & 0.0000 & 0.0338 & 0.0332 & 0.0332 & 0.0330 \\
75 & 25 & 0.0093 & 0.0047 & 0.0000 & 0.0000 & 0.0614 & 0.0599 & 0.0210 & 0.0208 \\
75 & 50 & 0.0025 & -0.0003 & 0.0000 & 0.0000 & 0.0152 & 0.0150 & 0.0149 & 0.0147 \\
75 & 75 & 0.0031 & -0.0011 & 0.0000 & 0.0000 & 0.0123 & 0.0123 & 0.0121 & 0.0120 \\
\hline Values autoregressive parameters: $\phi_{1}=0.3$ and $\phi_{2}=-0.4$. & & \\
\hline
\end{tabular}

Table 2.6: Results for the MLE in the first differenced model, ar(2). 
$E\left(\varepsilon_{i, t}\right)^{2}=\sigma^{2}=1$, the $c_{i}$ independently drawn from $N(1,3)$, the initial values independently drawn form $\left(y_{i,-1}, y_{i, 0}\right)^{\prime}=N\left(c_{i} /\left(1-\phi_{1}-\phi_{2}\right), V_{2}^{2}\right)$ where $V_{2}^{2}$ is given by $(2.56)$ and $\phi_{1}=0.3$ and $\phi_{2}=-0.4$. The results are given in the tables 2.5 and 2.6 .

Observe that the biases for the first coefficient $\phi_{1}$ appear to be more accurate than for $\phi_{2}$. Further the remarks made about the significance of the bias, even for larger $T$ also applies in this case, just like in the AR(1) case. The bias for the negative coefficient is, just like in the AR(1) case in practice larger than we might expect form Theorem 6.

Notice the sign of the small sample size biases of the asymptotically unbiased first differenced MLE in Table 2.6. The simulated estimators do closely mimic the theoretical. standard errors, even for small values of $T$ and $N$.

\subsection{Which estimator to use?}

As pointed out earlier in this chapter an important question is which estimator performs the best for a given pair of values of $T$ and $N$. Therefore we present in Table 2.7 the root. mean squared errors based on the biases and variances as found in the simulations.

As one can see the RMSE of the first differenced model is uniformly the smallest in all simulations. This indicates that the ML estimator based on the first differences is to be preferred for all combinations of $N$ and $T$ we simulated.

\begin{tabular}{|l|c|cc|ccc|c|c|}
\hline & & \multicolumn{2}{|c|}{ AR(1) } & \multicolumn{2}{c|}{ AR(1) } & \multicolumn{2}{c|}{ AR(2) } \\
\hline $\mathbf{T}$ & $\mathbf{N}$ & \multicolumn{2}{|c|}{$\phi_{1}=-\mathbf{0 . 6}$} & \multicolumn{2}{c|}{$\phi_{1}=\mathbf{0 . 6}$} & \multicolumn{1}{c|}{$\phi_{1}=\mathbf{0 . 3}, \phi_{2}=-\mathbf{0 . 4}$} \\
\hline & & direct & differenced & direct & differenced & direct & diferenced \\
\hline 10 & 10 & 0.0882 & 0.0822 & 0.1940 & 0.1115 & 0.1488 & 0.1374 \\
10 & 25 & 0.0812 & 0.0541 & 0.1638 & 0.0760 & 0.1154 & 0.0841 \\
10 & 50 & 0.0755 & 0.0447 & 0.1425 & 0.0713 & 0.1057 & 0.0665 \\
10 & 75 & 0.0697 & 0.0387 & 0.1491 & 0.0565 & 0.0940 & 0.0552 \\
25 & 10 & 0.0487 & 0.0466 & 0.0714 & 0.0537 & 0.0924 & 0.0846 \\
25 & 25 & 0.0422 & 0.0343 & 0.0608 & 0.0419 & 0.1005 & 0.0506 \\
25 & 50 & 0.0347 & 0.0252 & 0.0592 & 0.0304 & 0.0489 & 0.0367 \\
25 & 75 & 0.0338 & 0.0226 & 0.0559 & 0.0261 & 0.0444 & 0.0286 \\
50 & 10 & 0.0381 & 0.0367 & 0.0519 & 0.0424 & 0.0658 & 0.0632 \\
50 & 25 & 0.0252 & 0.0216 & 0.0353 & 0.0287 & 0.0411 & 0.0379 \\
50 & 50 & 0.0223 & 0.0186 & 0.0292 & 0.0193 & 0.0294 & 0.0251 \\
50 & 75 & 0.0200 & 0.0157 & 0.0267 & 0.0145 & 0.0270 & 0.0222 \\
75 & 10 & 0.0303 & 0.0296 & 0.0352 & 0.0296 & 0.0483 & 0.0477 \\
75 & 25 & 0.0175 & 0.0161 & 0.0254 & 0.0208 & 0.0330 & 0.0364 \\
75 & 50 & 0.0151 & 0.0133 & 0.0226 & 0.0147 & 0.0235 & 0.0215 \\
75 & 75 & 0.0127 & 0.0097 & 0.0206 & 0.0110 & 0.0202 & 0.0177 \\
\hline
\end{tabular}

Table 2.7: Root mean squared errors as found in the simulations. 


\subsection{Conclusions}

In this chapter we have analyzed Maximum Likelihood Estimation of AR(p) models in dynamic panel data. We derived the asymptotics of the MLE based on the first differenced model. The bias of the MLE based on the non-transformed model is derived and compared with the bias of the LSDV estimator. Further it is shown that the exact MLE in the transformed model can be calculated in an efficient way. How to extend these results to models which include exogenous variables is more complicated because of required additional assumptions about the exogenous variables.

We point out however that it is straightforward to extend the results for univariate processes as given in this chapter to multivariate autoregressive processes. This could lead to an alternative estimator for the instrumental variable method as introduced by Holz-Eakin et al. (1988).

Simulation results indicate further that the ML estimator based on the differenced model is to be preferred for reasonably large values of $T$. In our simulation even larger than 75 . We find that the ML estimator in the non transformed model a has serious bias even for $T=75$. This implies that in dynamic panel data the ML estimator applied to the model in first differences has to be preferred to the LSDV and ML estimator applied to the non-transformed model.

\subsection{Appendix}

In this appendix we will give the proofs of the results stated in the main text. Results which are important for proving the theorems are stated as lemmas.

\subsubsection{Proofs section 2.3.2}

Lemma 7 When $D_{T}$ is the $((T-1) \times T)$ first difference operator as defined in (2.15) and $V_{T}^{p},(T \times T), T \geq 2$ is the non-singular covariance matrix of a stationary autoregressive process of order $p$. Then

$$
\left(V_{T}^{p}\right)^{-1}-\frac{\left(V_{T}^{p}\right)^{-1} \iota_{T} t_{T}^{\prime}\left(V_{T}^{p}\right)^{-1}}{\iota_{T}^{i}\left(V_{T}^{p}\right)^{-1} \iota_{T}}=D_{T}^{i}\left(D_{T} V_{T}^{p} D_{T}^{\prime}\right)^{-1} D_{T}
$$

where $\iota_{T}$ is a $(T \times 1)$ vector of ones.

We note that this theorem is an extension of Theorem 1 of Lancaster and Lindenhovius (1996).

Proof. The proof is identical to the proof of Lancaster and Lindenhovius with the additional notice that $V_{T}^{p}$ is always non-singular for stationary AR(p) processes. From the fact that $V_{T}^{p}$ is non-singular it follows directly that the $((T-1) \times(T-1))$ matrix $D_{T} V_{T}^{p} D_{T}^{t}$ is non-singular, which implies that the matrices in (2.62) exist.

Let $L$ denote the left matrix and $R$ the right one. The proof is finished when we have proved $L=R$. Premultiply $L$ and $R$ by the non-singular matrix $V_{T}^{p}$ and postmultiply by 
$V_{T}^{p} D_{T}^{\prime}$ to obtain

$$
\begin{aligned}
V_{T}^{p} L V_{T}^{p} D_{T}^{\prime} & =V_{T}^{p} D_{T}^{*} \\
V_{T}^{p} R V_{T}^{p} D_{T}^{\prime} & =V_{T}^{p} D_{T}^{\prime}
\end{aligned}
$$

which implies, by the non-singularity of $V_{T}^{p}$

$$
L V_{T}^{p} D_{T}^{\prime}=R V_{T}^{p} D_{T}^{\prime}
$$

Since $L t_{T}=R t_{T}=0(2.65)$ implies that

$$
L\left[V_{T}^{p} D_{T}^{\prime}: \iota_{T}\right]=R\left[V_{T}^{p} D_{T}^{\prime}: \iota_{T}\right]
$$

and this implies $L=R$ when $\left[V_{T}^{p} D_{T}^{\prime}: t_{T}\right]$ is non-singular. "To proof this we note that this matrix is non-singular when

$$
V_{T}^{p} D_{T}^{\prime} c_{1}+\iota_{T} c_{2}=0 \Rightarrow c_{1}=c_{2}=0
$$

This follows directly when we premultiply (2.67) by $D_{T}$. Hence $L=R$.

We now give the proof of Lemma 1 on the concentrated log-likelihoods which is based on a special case of Lemma 7 .

Proof. (Lemma 1) We first prove (2.23). The likelihood is given by

$$
l^{J} \propto \frac{N}{2} \log \left(\left|\sigma^{-2}\left(V_{T+p}^{p}\right)^{-1}\right|\right)-\frac{1}{2 \sigma^{2}} \sum_{i=1}^{N}\left(y_{i}-\iota_{T+p} \mu_{i}\right)^{\prime}\left(V_{T+p}^{p}\right)^{-1}\left(y_{i}-\iota_{T+p} \mu_{i}\right) .
$$

We now concentrate out $\mu_{i}: \mu_{i}=\left[\iota_{T+p}^{\prime}\left(V_{T+p}^{p}\right)^{-1} \iota_{T+p}\right]^{-1} \iota_{T+p}^{\prime}\left(V_{T+p}^{p}\right)^{-1} y_{i}$. Define $C \equiv\left[\iota_{T+p}^{\prime}\left(V_{T+p}^{p}\right)^{-1} t_{T+p}\right]^{-1}$, then we obtain $\mu_{i} \equiv C \iota_{T+p}^{\prime}\left(V_{T+p}^{p}\right)^{-1} y_{i}$ and we obtain

$$
\begin{aligned}
& \left(y_{i}-\iota_{T+p} \mu_{i}\right)^{\prime}\left(V_{T+p}^{p}\right)^{-1}\left(y_{i}-\iota_{T+p} \mu_{i}\right) \\
= & y_{i}^{\prime}\left(I-C \iota_{T+p} \iota_{T+p}^{\prime}\left(V_{T+p}^{p}\right)^{-1}\right)^{\prime}\left(V_{T+p}^{p}\right)^{-1}\left(I-C \iota_{T+p} \iota_{T+p}^{\prime}\left(V_{T+p}^{p}\right)^{-1}\right) y_{i} \\
= & y_{i}^{\prime}\left(I-C \iota_{T+p} \iota_{T+p}^{\prime}\left(V_{T+p}^{p}\right)^{-1}\right)^{\prime} \\
& \times\left(\left(V_{T+p}^{p}\right)^{-1}-C\left(V_{T+p}^{p}\right)^{-1} \iota_{T+p} \iota_{T+p}^{\prime}\left(V_{T+p}^{p}\right)^{-1}\right) y_{i} \\
= & y_{i}^{\prime}\left(\left(V_{T+p}^{p}\right)^{-1}-2 C\left(V_{T+p}^{p}\right)^{-1} \iota_{T+p} \iota_{T+p}^{\prime}\left(V_{T+p}^{p}\right)^{-1}\right) y_{i} \\
& +y_{i}^{\prime}\left(C^{2}\left(V_{T+p}^{p}\right)^{-1} \iota_{T+p} \iota_{T+p}^{\prime}\left(V_{T+p}^{p}\right)^{-1} \iota_{T+p} \iota_{T+p}^{\prime}\left(V_{T+p}^{p}\right)^{-1}\right) y_{i}^{\prime} \\
= & y_{i}^{\prime}\left(\left(V_{T+p}^{p}\right)^{-1}-C\left(V_{T+p}^{p}\right)^{-1} \iota_{T+p} \iota_{T+p}^{\prime}\left(V_{T+p}^{p}\right)^{-1}\right) y_{i}
\end{aligned}
$$

which gives applying Lemma 7

$$
\tilde{l}^{\jmath} \propto \frac{N}{2} \log \left(\left|\sigma^{-2}\left(V_{T+p}^{p}\right)^{-1}\right|\right)-\frac{1}{2 \sigma^{2}} \sum_{i=1}^{N} y_{i}^{\prime} D_{T+p}^{z}\left(\sigma^{-2}\left(D_{T+p} V_{T+p}^{p} D_{T+p}^{\prime}\right)^{-1} D_{T+p} y_{i} .\right.
$$


Observing that the constant term has not changed we obtain the stated result.

For the concentrated log-likelihood

$$
\begin{aligned}
& l^{C}=-\frac{N T}{2} \log (2 \pi)+\frac{N T}{2} \log \left|\sigma^{-2}\right| \\
& -\frac{1}{2 \sigma^{2}} \sum_{i=1}^{N} \sum_{i=1}^{T}\left(\left(y_{i, t}-\mu_{i}\right)-\phi_{1}\left(y_{i, t-1}-\mu_{i}\right)-\ldots-\phi_{p}\left(y_{i, t-p}-\mu_{i}\right)\right)^{2}
\end{aligned}
$$

we note that

$$
\frac{\partial l^{C}}{\partial \mu_{i}}=\frac{\left(1-\phi_{2}-\ldots-\phi_{p}\right)}{\sigma^{2}} \sum_{t=1}^{T}\left(\left(y_{i, t}-\mu_{i}\right)-\phi_{1}\left(y_{i, t-1}-\mu_{i}\right)-\ldots-\phi_{p}\left(y_{i, t-p}-\mu_{i}\right)\right),
$$

putting this equal to zero and solving for $\mu_{i}$ gives

$$
\mu_{i}=\frac{1}{T} \frac{1}{\left(1-\phi_{1}-. .-\phi_{p}\right)} \sum_{t=1}^{T}\left(y_{i, t}-\phi_{1} y_{i, t-1}-\ldots-\phi_{p} y_{i, t-p}\right) .
$$

Substituting this back into (2.72) gives the result as stated.

We now prove Lemma 2.

Proof. (Lemma 2) By (2.19) we have

$$
l_{F D}^{C} \propto \frac{N}{2} \log \left|\sigma^{-2}\left(D_{T} D_{T}^{\prime}\right)^{-1}\right|-\frac{1}{2 \sigma^{2}} \sum_{i=1}^{N} y_{i}^{\prime} A_{T}^{p q} D_{T}^{\prime}\left[\left(D_{T} D_{T}^{\prime}\right)^{-1}\right] D_{T} A_{T}^{p} y_{i} .
$$

Now note that by Lemma 7

$$
D_{T}^{\prime}\left[\left(D_{T} D_{T}^{\prime}\right)^{-1}\right] D_{T}=I-\frac{1}{T} \iota_{T} \iota_{T}^{\prime}
$$

which is just the operator which changes a $(T \times 1)$ vector in a $(T \times 1)$ vector with as elements the deviations from the original vector to the mean of the vector. The result stated in the Lemma follows immediately.

The following two lemmas also are a generalization of a result by Lancaster and Lindenhovius (1996) from the $\mathrm{AR}(1)$ case to the $\mathrm{AR}(\mathrm{p})$ case. We will use these results when we analyze the differences between the log-likelihoods.

Lemma 8 When $D_{T}$ is the $((T-1) \times T)$ first difference operator as defined in (2.15) and $V_{T}^{p},(T \times T), T \geq 2$, is the nonsingular covariance matrix of a stationary autoregressive process of order $p$. Then

$$
\left|D_{T} V_{T}^{p} D_{T}^{\prime}\right|=\left|V_{T}^{p}\right|\left(t_{T}^{\prime}\left(V_{T}^{p}\right)^{-1} \iota_{T}\right)
$$

Observe that (2.77) is undefined for $T=1$.

Proof. Similar to Lancaster and Lindenhovius (1996). Consider the augmented matrix

$$
M=\left(\begin{array}{c}
D_{T} \\
\iota_{T}^{\prime}
\end{array}\right) V_{T}^{p}\left(\begin{array}{ll}
D_{T}^{\prime} & \iota_{T}
\end{array}\right)=\left(\begin{array}{cc}
D_{T} V_{T}^{p} D_{T}^{\prime} & D_{T} V_{T}^{p} t_{T} \\
\iota_{T}^{\prime} V_{T}^{p} D_{T}^{\prime} & \iota_{T}^{\prime} V_{T}^{p} \iota_{T}
\end{array}\right)
$$


Then observe that $\left|\left(D_{T}^{t}: u_{T}\right)\right|=T$ from which we obtain

$$
|M|=T^{2}\left|V_{T}^{p}\right|
$$

From the standard formula for the determinant of a partitioned matrix, see Lütkepohl (1996),

$$
|M|=\left|D_{T} V_{T}^{p} D_{T}^{\prime}\right|\left|\iota_{T}^{\prime}\left[V_{T}^{p}-V_{T}^{p} D_{T}^{\prime}\left(D_{T} V_{T}^{p} D_{T}^{\prime}\right)^{-1} D_{T} V_{T}^{p}\right] t_{T}\right|
$$

which equals by (2.62) $\iota_{T} \iota_{T}^{\prime} / \iota_{T}^{\prime} V_{T}^{p-1} \iota_{T}$. Hence

$$
\begin{gathered}
|M|=\left|D_{T} V_{T}^{p} D_{T}^{\prime}\right|\left|\iota_{T}^{\prime}\left[\iota_{T} \iota_{T}^{\prime} / \iota_{T}^{\prime}\left(V_{T}^{p}\right)^{-1} \iota_{T}\right] \iota_{T}\right| . \\
|M|=\frac{\left|D_{T} V_{T}^{p} D_{T}^{\prime}\right| T^{2}}{\iota_{T}^{\prime}\left(V_{T}^{p}\right)^{-1} \iota_{T}} .
\end{gathered}
$$

Now using (2.79) the stated result follows.

The following corollary was given as Theorem 2 in Lancaster and Lindenhovius.

Corollary 9 When $D_{T}$ is the $((T-1) \times T)$ first difference operator as defined in 2.15 and $V_{T}^{1}$ : $(T \times T), T \geq 2$ is the non singular covariance matrix of a stationary $A R(1)$ process. Then

$$
\left|D_{T} V_{T}^{p} D_{T}^{\prime}\right|=\frac{T\left(1-\phi_{1}\right)+2 \phi_{1}}{1+\phi_{1}}
$$

It can also easily be obtained applying the following result in case $p=1$.

Lemma 10 Let $V_{T}^{p}$ be the covariance of a stationary $A R(p)$ process for $T$ abservations then we have for all $T \geq p \geq 1$

$$
\begin{gathered}
\left|V_{T}^{p}\right|=\left|V_{p}^{p}\right|, \\
\left(\iota_{T}^{\prime}\left(V_{T}^{p}\right)^{-1} \iota_{T}\right)=\left(\iota_{p}^{\prime}\left(V_{p}^{p}\right)^{-1} \iota_{p}+(T-p)\left(1-\phi_{1}-. .-\phi_{p}\right)^{2}\right),
\end{gathered}
$$

and

$$
\frac{\partial\left(\iota_{T}^{\prime}\left(V_{T}^{p}\right)^{-1} \iota_{T}\right)}{\partial \phi^{\prime}}=\operatorname{vec}\left(\iota_{T} \iota_{T}^{\prime}\right)^{\prime} \frac{\partial\left(V_{p}^{p}\right)^{-1}}{\partial \phi^{\prime}}-2(T-p)\left(\left(1-\iota_{\mathrm{p}}^{\prime} \phi\right)^{\prime} \iota_{p}\right)
$$

where $\phi=\left(\phi_{1}, . ., \phi_{p}\right)^{\prime}$ are the lag coefficients of the $A R(p)$ process.

Proof. First we observe that it is well known that

$$
\left|V_{T}^{p}\right|=\left|V_{p}^{p}\right| \text {. }
$$

See for instance Galbraith and Galbraith (1974). To calculate $\iota_{T}^{\prime}\left(V_{T}^{p}\right)^{-1} \iota_{T}$ we use the following well known identity

$$
x_{1, T}^{\prime}\left(V_{T}^{p}\right)^{-1} x_{1, T}=x_{1, p}^{\prime}\left(V_{p}^{p}\right)^{-1} x_{1, p}+\sum_{t=p+1}^{T}\left(x_{t}-\phi_{1} x_{t-1}-. .-\phi_{p} x_{t-p}\right)^{2}
$$


where $x_{1, x^{\prime}}$ is the ( $T \times 1$ vector of all observations $x_{1, p}$, the $p \times 1$ vector of initial observations and $x_{t}$ the observation at time $t$. This relation can easily be derived from the factorization of the likelihood. We observe that this relation also holds when we replace the $x$-vectors by vectors consisting of ones and obtain

$$
\iota_{T}\left(V_{T}^{p}\right)^{-1} \iota_{T}=\iota_{p}^{\prime} V_{p}^{p-1} \iota_{p}+(T-p)\left(1-\phi_{1}-\ldots-\phi_{p}\right)^{2}
$$

which gives $(2.85)$. For $(2.86)$ we observe

$$
\begin{aligned}
\frac{\partial\left(\iota_{T}^{\prime}\left(V_{T}^{p}\right)^{-1} \iota_{T}\right)}{\partial \phi^{\prime}} & =\frac{\partial\left(\iota_{p}^{\prime}\left(V_{p}^{p}\right)^{-1} \iota_{p}+(T-p)\left(1-\phi_{1}-\ldots-\phi_{p}\right)^{2}\right)}{\partial \phi^{\prime}} \\
& =\frac{\partial\left(\iota_{p}^{\prime}\left(V_{p}^{p}\right)^{-1} \iota_{p}\right)}{\partial \phi^{\prime}}+(T-p) \frac{\partial\left(1-\iota_{p}^{\prime} \phi^{\prime}\right)^{\prime}\left(1-\iota_{p}^{\prime} \phi\right)}{\partial \phi^{\prime}} \\
& =\operatorname{vec}\left(\iota_{p} \iota_{p}^{\prime}\right)^{\prime} \frac{\partial\left(V_{p}^{p}\right)^{-1}}{\partial \phi^{\prime}}-2(T-p)\left(\left(1-\iota_{p}^{\prime} \phi\right)^{\prime} \iota_{p}\right)
\end{aligned}
$$

This completes the proof.

The following corollary will be needed in several of the results derived later on.

Corollary 11 In the same notation as in the previous lemma we have for $T \geq p \geq 1$

$$
\frac{\partial \log \left(\iota_{T}^{\prime}\left(V_{T}^{p}\right)^{-1} \iota_{T}\right)}{\partial \phi^{\prime}}=\frac{\operatorname{vec}\left(\iota_{p} \iota_{p}^{\prime}\right)^{\prime} \frac{\partial\left(V_{p}^{p}\right)^{-1}}{\partial \phi^{\prime}}-2(T-p)\left(\left(1-\iota_{p}^{\prime} \phi\right)^{\prime} \iota_{p}\right)}{\iota_{p}^{\prime}\left(V_{p}^{p}\right)^{-1} \iota_{p}+(T-p)\left(1-\phi_{1}-\ldots-\phi_{p}\right)^{2}} .
$$

This Corollary states that when $T \rightarrow \infty$ the expression converges to a nonzero constant.

Proof. (Theorem 3) For the likelihoods with stochastic initial observations we use the equality

$$
\begin{aligned}
& \frac{N}{2} \log \left(\left|\sigma^{-2}\left(D_{T+p} V_{T+p}^{p} D_{T+p}^{\prime}\right)^{-1}\right|\right) \\
= & -\frac{N(T+p-1)}{2} \log \left(\sigma^{2}\right)-\frac{N}{2} \log \left(\left|V_{T+p}^{p}\right|\left(\iota_{T+p}^{\prime}\left(V_{T+p}^{p}\right)^{-1} \iota_{T+p}\right)\right) \\
= & -\frac{N(T+p-1)}{2} \log \left(\sigma^{2}\right)+\frac{N}{2} \log \left(\left|V_{T+p}^{p}\right|^{-1}\right) \\
& -\frac{N}{2} \log \left(\iota_{T+p}^{\prime}\left(V_{T+p}^{p}\right)^{-1} \iota_{T+p}\right)
\end{aligned}
$$

which follows with Lemma 8 above. Then again apply the Lemmas 1 and 2 and the result follows. 


\subsubsection{Proofs section 2.3.3}

Lemma 12 The Hessian of

$$
\frac{N}{2} \log \left(\sigma^{2}\right)-\frac{N}{2} \log \left(t_{T+p}^{\prime}\left(V_{T+p}^{p}\right)^{-1} t_{T+p}\right)
$$

is given by

$$
\left[\begin{array}{cc}
-\frac{N}{2} \frac{\partial^{2} \log \left(i_{T+p}^{2}\left(v_{T+p}^{2}\right)^{-1} e_{T+p}\right)}{\partial \phi \partial \phi^{2}} & 0 \\
0 & -\frac{N}{2 \sigma^{4}}
\end{array}\right]
$$

with

$$
\frac{\partial^{2} \log \left(t_{T+p}^{\prime}\left(V_{T+p}^{p}\right)^{-1} t_{T+p}\right)}{\partial \phi \partial \phi^{\prime}}=\frac{\partial^{2} \log \left(\iota_{p}^{\prime}\left(V_{p}^{p}\right)^{-1} \iota_{p}+T\left(1-\phi_{1}-. .-\phi_{p}\right)^{2}\right)}{\partial \phi \partial \phi^{\prime}} .
$$

Proof. Define

$$
R=\frac{N}{2} \log \left(\sigma^{2}\right)-\frac{N}{2} \log \left(t_{T+p}^{t}\left(V_{T+p}^{p}\right)^{-1} \iota_{T+p}\right)
$$

Then it follows from the fact that $V_{T+p}^{p}$ does not depend on $\sigma^{2}$ that $\frac{\partial^{2} R}{\partial \phi^{2} \partial \sigma^{2}}=0$ and $\frac{\partial^{2} R}{\partial \sigma^{2} \partial \sigma^{2}}=-\frac{N}{2 \sigma^{4}}$. Using (2.89) the stated result directly follows.

As we have obtained the relation between the log-likelihood of the direct model concentrated with respect to $\mu_{i}$ and the log-likelihood of the first differenced model in Theorem 5 we can construct derivatives, estimators, variance etc. by calculating either one and using the lemma stated directly above. We therefore choose the one most convenient to evaluate.

Lemma 13 The Fisher Information matrix of the log-likelihood $l^{J}$ is given by

$$
I^{\jmath}=\left[\begin{array}{ccc}
\operatorname{diag}\left(I_{\mu_{1} \mu_{1}}, \ldots, I_{\mu_{N} \mu_{N}}\right) & 0 & 0 \\
0 & I_{\phi \phi^{\prime}} & I_{\sigma^{2} \phi^{\prime}} \\
0 & I_{\phi \sigma^{2}} & I_{\sigma^{2} \sigma^{2}}
\end{array}\right]
$$

where

$$
\begin{aligned}
I_{\phi \phi^{\prime}}= & \frac{T}{T+p} V_{p}^{p}+\frac{1}{2(T+p)}\left|\left(V_{p}^{p}\right)\right|^{-2} \frac{\partial\left|\left(V_{p}^{p}\right)\right|}{\partial \phi} \frac{\partial\left|\left(V_{p}^{p}\right)\right|}{\partial \phi^{\prime}} \\
& +\frac{1}{2(T+p)} \frac{\partial \operatorname{vec}\left(\left(V_{p}^{p}\right)^{-1}\right)^{\prime}}{\partial \phi}\left(V_{p}^{p} \otimes V_{p}^{p}\right) \frac{\partial \operatorname{vec}\left(\left(V_{p}^{p}\right)^{-1}\right)}{\partial \phi^{\prime}} \\
I_{\sigma^{2} \sigma^{2}}= & \frac{1}{2 \sigma^{4}} \\
I_{\sigma^{2} \phi^{\prime}}= & \frac{1}{2 \sigma^{2}(T+p)} \operatorname{vec}\left(V_{p}^{p}\right)^{\prime} \frac{\partial \operatorname{vec}\left(\left(V_{p}^{p}\right)^{-1}\right)}{\partial \phi^{\prime}}
\end{aligned}
$$

For the individual effects

$$
I_{\mu_{i} \mu_{i}}=-\frac{1}{N(T+p) \sigma^{2}} L_{p}^{\prime \prime}\left[\left(V_{p}^{p}\right)^{-1}\right] \iota_{p}+\frac{T}{N(T+p)} \frac{\left(1-\phi_{1}-\ldots-\phi_{p}\right)^{2}}{\sigma^{2}}
$$

all ather blocks of the Fisher Information equal zero. 
Proof. The (average) Fisher Information matrix is given by $\frac{1}{N(T+p)} E\left(-H\left(l^{J}\right)\right)$, where $H(\bullet)$ indicates the Hessian of the log-likelihood. The first order derivative of the likelihood with respect to $\mu_{i}$ is given by

$$
\begin{aligned}
\frac{\partial l^{J}}{\partial \mu_{i}}= & \frac{1}{\sigma^{2}}\left(y_{i, 0}-\mu_{i}\right)^{r}\left[\left(V_{p}^{p}\right)^{-1}\right] \iota_{p}+ \\
& \frac{\left(\mathbb{1}-\phi_{1}-\ldots, \phi_{p}\right)}{\sigma^{2}} \sum_{t=1}^{T}\left(\left(y_{i, t}-\mu_{i}\right)-\phi_{1}\left(y_{i, t-1}-\mu_{i}\right)-\ldots-\phi_{p}\left(y_{i, t-p}-\mu_{i}\right)\right) .
\end{aligned}
$$

It is now easy to verify that

$$
\frac{\partial^{2} l^{J}}{\partial \mu_{i}^{2}}=\frac{1}{\sigma^{2}} e^{r}\left[\left(V_{p}^{p}\right)^{-1}\right] \iota_{p}-T \frac{\left(1-\phi_{1}-\ldots-\phi_{p}\right)^{2}}{\sigma^{2}}
$$

that $\frac{\partial^{2} l_{D}^{g}}{\partial \mu_{i} \partial \mu_{j}}=0$, for $i \neq j, E\left(\frac{\partial^{2} l^{J}}{\partial \mu_{i} \partial \sigma^{2}}\right)=0$ and finally

$$
\frac{\partial^{2} l^{J}}{\partial \mu_{i} \partial \phi^{\prime}}=\frac{1}{\sigma^{2}}\left(\left(\left(y_{i, 0}-\mu_{i}\right), \ldots,\left(y_{i,-p+1}-\mu_{i}\right)\right) \otimes \iota_{p}\right) \frac{\partial \operatorname{vec}\left(\left(V_{p}^{p}\right)^{-1}\right)}{\partial \phi^{\prime}}
$$

which implies $E\left(\frac{\partial^{2} l^{s}}{\partial \mu_{i} \partial \phi^{\prime}}\right)=0$ by the fact that $\frac{\partial v e c\left(\left(V_{i p}^{P}\right)^{-1}\right)}{\partial \phi^{\prime}}$ is nonrandom. We further have

$$
\begin{aligned}
\frac{\partial l^{J}}{\partial \phi \partial \phi^{\prime}}= & -\frac{N}{2}\left|\left(V_{p}^{p}\right)\right|^{-2} \frac{\partial\left|\left(V_{p}^{p}\right)\right|}{\partial \phi} \frac{\partial\left|\left(V_{p}^{p}\right)\right|}{\partial \phi^{\prime}} \\
& -\frac{1}{2 \sigma^{4}} \sum_{i=1}^{N} \frac{\partial v e c\left(\left(V_{p}^{p}\right)^{-1}\right)^{\prime}}{\partial \phi}\left(W_{i} \otimes W_{i}\right) \frac{\partial v e c\left(\left(V_{p}^{p}\right)^{-1}\right)}{\partial \phi^{\prime}} \\
& -\frac{1}{\sigma^{2}} \sum_{i=1}^{N} \sum_{t=1}^{T}\left(\left(y_{i, t}-\mu_{i}\right)_{,} \ldots\left(y_{i, t-p}-\mu_{i}\right)\right)^{\prime}\left(\left(y_{i, t}-\mu_{i}\right)_{,} \ldots,\left(y_{i, t-p}-\mu_{i}\right)\right)
\end{aligned}
$$

where $W_{i}=\left(\left(\left(y_{i, 0}-\mu_{i}\right), \ldots,\left(y_{i,-p+1}-\mu_{i}\right)\right)\left(\left(y_{i, 0}-\mu_{i}\right), \ldots\left(y_{i_{1}-p+1}-\mu_{i}\right)\right)^{\prime}\right)$. Taking expectations and averaging leads to

$$
\begin{aligned}
& E\left(\frac{1}{N(T+p)} \frac{\partial l^{J}}{\partial \phi \partial \phi^{\prime}}\right) \\
= & -\frac{T}{T+p} V_{p}^{p} \\
& -\frac{1}{2(T+p)}\left|\left(V_{(p)}^{p}\right)\right|^{-2} \frac{\partial\left|\left(V_{p}^{p}\right)\right|}{\partial \phi} \frac{\partial\left|\left(V_{p}^{p}\right)\right|}{\partial \phi^{\prime}} \\
& -\frac{1}{2(T+p)} \frac{\partial \operatorname{vec}\left(\left(V_{p}^{p}\right)^{-1}\right)^{\prime}}{\partial \phi}\left(V_{p}^{p} \otimes V_{p}^{p}\right) \frac{\partial \operatorname{vec}\left(\left(V_{p}^{p}\right)^{-1}\right)}{\partial \phi^{\prime}} .
\end{aligned}
$$

Finally we have

$$
\begin{aligned}
\frac{\partial l^{J}}{\partial \sigma^{2} \partial \sigma^{2}}= & \frac{N(T+p)}{2} \frac{1}{\sigma^{4}}-\frac{1}{\sigma^{6}} \sum_{i=1}^{N}\left(y_{i, 0}-\mu_{i}\right)^{\prime}\left[\left(V_{p}^{p}\right)^{-1}\right]\left(y_{i, 0}-\mu_{i}\right) \\
& -\frac{1}{\sigma^{6}} \sum_{i=1}^{N} \sum_{t=1}^{T}\left(\left(y_{i, t}-\mu_{i}\right)-\phi_{1}\left(y_{i, t-1}-\mu_{i}\right)-\ldots-\phi_{p}\left(y_{i, t-p}-\mu_{i}\right)\right)^{2},
\end{aligned}
$$


taking expectations and normalizing gives

$$
E\left(\frac{1}{N(T+p)} \frac{\partial^{2} l^{J}}{\partial \sigma^{2} \partial \sigma^{2}}\right)=-\frac{1}{2 \sigma^{4}} .
$$

Here we used the fact that, under the true parameters,

$$
\left(y_{i, 0}-\mu_{i}\right)^{t}\left[\left(V_{p}^{p}\right)^{-1}\right]\left(y_{i, 0}-\mu_{i}\right) \sim \sigma^{2} \mathcal{X}_{p}^{2}
$$

and

$$
\sum_{i=1}^{N} \sum_{t=1}^{T}\left(\left(y_{i, t}-\mu_{i}\right)-\phi_{1}\left(y_{i, t-1}-\mu_{i}\right)-\ldots-\phi_{p}\left(y_{i, t-p}-\mu_{i}\right)\right)^{2} \sim \sigma^{2} \mathcal{X}_{N T}^{2} .
$$

For the cross term we have

$$
\begin{aligned}
\frac{\partial^{2} l^{J}}{\partial \sigma^{2} \partial \phi^{\prime}}= & -\frac{1}{2 \sigma^{4}} \sum_{i=1}^{N} \operatorname{vec}\left(W_{i}\right)^{\prime} \frac{\partial \operatorname{vec}\left(\left(V_{p}^{p}\right)^{-1}\right)}{\partial \phi^{t}} \\
& -\frac{1}{\sigma^{4}} \sum_{i=1}^{N} \sum_{t=1}^{T}\left(\left(y_{i, t}-\mu_{i}\right)-\phi_{1}\left(y_{i, t-1}-\mu_{i}\right)-\ldots-\phi_{p}\left(y_{i, t-p}-\mu_{i}\right)\right) \\
& \times\left(\left(y_{i, t-1}-\mu_{i}\right), \ldots,\left(y_{i, t-p}-\mu_{i}\right)\right) .
\end{aligned}
$$

Now normalizing and taking expectations leads to

$$
E\left(\frac{1}{N(T+p)} \frac{\partial^{2} l^{J}}{\partial \sigma^{2} \partial \phi^{\prime}}\right)=-\frac{1}{2 \sigma^{2}(T+p)} \operatorname{vec}\left(V_{p}^{p}\right)^{\prime} \frac{\partial v e c\left(\left(V_{p}^{p}\right)^{-1}\right)}{\partial \phi^{\prime}} .
$$

Observe that when $T$ is kept finite this term does not disappear.

We are now ready to prove Theorem 6.

Proof. (Theorem 6) As is well known the variance of the estimators for $\phi$ and $\sigma^{2}$ based on the concentrated likelihood is equal to the variance of the direct likelihood. The variance is proportional to the inverse of the information matrix. As a consequence of Theorem 3 it follows that the Fisher Information for the first differenced model, with random initial observations is given by

$$
\begin{aligned}
I_{F D}^{J}= & {\left[\begin{array}{ll}
I_{\phi \phi^{\prime}} & I_{\sigma^{2} \phi^{\prime}} \\
I_{\phi \sigma^{2}} & I_{\sigma^{2} \sigma^{2}}
\end{array}\right] } \\
& -\frac{1}{N(T+p)} H\left(\frac{N}{2} \log \left(\sigma^{2}\right)-\frac{N}{2} \log \left({t_{T+p}^{\prime}}_{T}^{\prime}\left(V_{T+p}^{p}\right)^{-1}{ }_{{ }^{\prime}+p}\right)\right),
\end{aligned}
$$

where the information matrix is given by $(2.97)$ and $H(\bullet)$ is denotes again the Hessian matrix at - Note that this relation only holds due to the mere coincidence that all components are independent of $\mu_{i}$.

Substituting the right expression into (2.112) and rearrange a bit we obtain

$$
I_{F D}^{J}=\left[\begin{array}{cc}
\frac{T}{T+p} V_{p}^{p} & 0 \\
0 & \frac{1}{2 \sigma^{4}}
\end{array}\right]+\frac{1}{2(T+p)} I C_{p}
$$


where

$$
I C_{p}=\left[\begin{array}{cc}
I C_{\phi \phi^{\prime}} & \frac{1}{\sigma^{2}} \operatorname{vec}\left(V_{p}^{p}\right)^{\prime} \frac{\left.\partial \operatorname{vec}\left(V_{p}^{p}\right)^{-1}\right)}{\partial \phi^{\prime}} \\
\frac{1}{\sigma^{2}} \frac{\partial \operatorname{vec}\left(\left(V_{p}^{p}\right)^{-1}\right)^{\prime}}{\partial \phi} \operatorname{vec}\left(V_{p}^{p}\right) & -\frac{1}{\sigma^{4}}
\end{array}\right]
$$

with

$$
\begin{aligned}
I C_{\phi \phi^{\prime}}= & \left|\left(V_{p}^{p}\right)\right|^{-2} \frac{\partial\left|\left(V_{p}^{p}\right)\right|}{\partial \phi} \frac{\partial\left|\left(V_{p}^{p}\right)\right|}{\partial \phi^{\prime}} \\
& +\frac{1}{\sigma^{2}} \frac{\partial \operatorname{vec}\left(\left(V_{p}^{p}\right)^{-1}\right)^{\prime}}{\partial \phi}\left(V_{p}^{p} \otimes V_{p}^{p}\right) \frac{\left.\partial \operatorname{vec}\left(V_{p}^{p}\right)^{-1}\right)}{\partial \phi^{\prime}} \\
& +\frac{\partial^{2} \log \left(\iota_{p}^{\prime}\left(V_{p}^{p}\right)^{-1} \iota_{p}+T\left(1-\phi_{1}-. .-\phi_{p}\right)^{2}\right)}{\partial \phi \partial \phi^{\prime}} .
\end{aligned}
$$




\section{Chapter 3}

\section{Forecasting with Dynamic Panel Data Models: To Pool or not to Pool.}

\subsection{Introduction}

In the previous chapter we studied the behaviour of estimators in dynamic panel data models, i.e in models where we allow some parameters to be different for each entitiy. Due to this assumption the behaviour of estimators depends on the ratio of the number of entities to the number of observed time periods. We saw this in the previous chapter. This problem does not occur when we restrict all parameters to be the same accross entities. Although the restriction is severe, it is used in many applied studies. Moreover in many applications this assumption seems to work very well. Forecasting is one of the examples where imposing pooling restrictions, restricting all parameters to be the same accross entities, leads to better results. In this chapter we will study this last phenomenon in detail.

We analyze the issue of pooling models and using these models for forecasting for a given set of $N$ individual units observed over $T$ periods of time. Large $T$ asymptotics, with $N$ fixed, provide the benchmark against which to evaluate the methods considered ${ }^{1}$. Pooling estimates in panel data models is appropriate if parameters are the same for the individual units observed. When the parameters are different but exhibit some similarity, pooling may also lead to a reduction of the mean squared error (MSE) of the estimates. A reduction of the MSE will be achieved when the square of the bias resulting from imposing false restrictions is outweighed by the reduction of the variance of the estimator due to restricted estimation. The existence of this trade-off has generated the literature on MSE criteria and associated tests for the superiority of restricted over unrestricted least squares estimators [see e.g. Wallace and Toro-Vizcarrondo (1969), Wallace (1972), Goodnight and Wallace (1972) and McElroy (1977)].

\footnotetext{
${ }^{1}$ In (macro)economic data sets, it often happens that a given number of entities is observed over a longer period of time whereas traditionally, panel data are available for a large and variable number of entities observed for a fixed number of time periods [see e.g. Baltagi (1995) for a recent owerview, Maddala (1991) and Maddala et al. (1994)].
} 
Pooling techniques have, for instance, been successfully applied to test the market efficiency hypothesis [see e.g. Bilson (1981)] and to forecast multi-country output growth rates. In a study of real GNP growth rates of nine OECD countries for the period 19511981 Garcia-Ferrer et al. (1987) showed that pooled estimates of an AR(3) model with leading indicator variables provided superior forecasting results. Forecasting results for an extended time period, 1974-1984, and an extended number of countries, eighteen OECD countries, provided by Zellner and Hong (1989) were in favor of the earlier findings.

Leading economic indicators have come to play a dominant role in forecasting business cycle turning points on a single country level (Stock and Watson, 1989) as well as on a multi country level (Zellner and Min, 1991). Finally we note that cross-country, crossequation restrictions have also been imposed to analyze convergence of annual log real per capita output for 15 OECD countries from 1900 to 1987 (Bernard and Durlauf, 1995).

The objective of this chapter is threefold. First, in section 2, we investigate theoretically whether the improvement of forecasting performance using pooling techniques instead of single-country forecasts remains valid as $T$ grows large(r) while $N$ remains constant. The model that we investigate consists of a set of dynamic regression equations with contemporaneously correlated disturbances. It nests the specifications of GarciaFerrer et al. (1987). Second, in section 3 we present simulation results which give some insights in the importance of the gains of pooling data when sample size is small. They also provide evidence on the statistical properties of some test procedures for pooling restrictions. Third, the theoretical results are investigated empirically. The Zellner and Min (1991) data were slightly modified to have a consistent set of real GDP growth rates of 18 OECD countries for an extended period of 1948 to 1990 , to forecast international growth rates using individual and pooled estimates of the AR(3)II model. Section 4 concludes.

\subsection{The model and the forecasting procedures}

Consider a linear regression model for $N$ units/countries and $T$ successive observations :

$$
y_{i, t}=x_{i, t}^{\prime} \beta_{i}+\varepsilon_{i, t} \quad i=1,2, \ldots N, t=1,2, \ldots T
$$

or

$$
\underset{(T \times 1)}{y_{i}}=\underset{(T \times k)(k \times 1)}{X_{i}} \beta_{i}+\underset{(T \times 1)}{\varepsilon_{i}}, \quad i=1,2, \ldots N
$$

where $y_{i, t}$ denotes the value of the endogenous variable $y$ for country $i$ in period $t, x_{i, t}$ is a column vector of $k$ explanatory variables, $\beta_{i}$ is a column vector of $k$ regression coefficients for country $i$ and $\varepsilon_{i, t}$ denotes a disturbance term. Alternatively, the model (3.2) for $N$ countries can be written as

$$
\underset{(n \times 1)}{y}=\underset{(n \times N k)}{X_{(N k \times 1)}^{*}} \underset{(n \times 1)}{\beta_{k}}
$$

with $y=\left(y_{1}^{\prime}, y_{2}^{\prime}, \ldots, y_{N}^{\prime}\right)^{\prime}, n=T N, X^{*}=\operatorname{diag}\left(X_{1}, X_{2}, \ldots, X_{N}\right)$ $\beta_{a}=\left(\beta_{1}^{\prime}, \beta_{2}^{\prime}, \ldots, \beta_{N}^{\prime}\right)^{\prime}$ and $\varepsilon=\left(\varepsilon_{1}^{\prime}, \varepsilon_{2}^{\prime}, \ldots, \varepsilon_{N}^{\prime}\right)^{\prime}$.

We allow the vector $x_{i, t}$ to contain lagged values of $y_{i, i}$. The disturbances $\varepsilon_{i, t}$ are assumed to be normally distributed with mean zero, serially uncorrelated but possibly 
contemporaneously correlated, that is $\varepsilon \sim N(0, \Omega)$ with $\Omega=\Sigma \otimes I_{r}$ where $\Sigma$ denotes the contemporaneous covariance matrix of dimension $N$. The regressors $x_{i, t}$ are predetermined, $E\left(x_{i, t} \varepsilon_{j, s}^{\prime} \mid x_{\ell, t-1}, x_{\varepsilon_{i, t-2}}, \ldots, \ell=1,2, \ldots N\right)=0$ for all $i, j$ and $t \leq s$.

We consider the following one-step-ahead forecasts:

1. The individual forecast based on the least squares estimator of $\beta_{i}, \hat{\beta}_{i}=\left(X_{i}^{\prime} X_{i}\right)^{-1} X_{i}^{\prime} y_{i}$ which leads to the forecast

$$
\hat{y}_{i, T+1}=x_{i, T+1}^{v} \hat{\beta}_{i} .
$$

2. The pooled forecast is

$$
\hat{y}_{i, T+1}^{p}=x_{i, T+1}^{\prime} \hat{\beta}^{p}
$$

with $\hat{\beta}^{P}$ being the OLS estimator for the pooled data $\hat{\beta}^{p}=\left(X^{\prime} X\right)^{-1} X^{\prime} y$ where $X=$ $\left(X_{1}^{\prime}, X_{2}^{\prime}, \ldots, X_{N}^{\prime}\right)^{\prime}$.

3. The forecast based on a feasible seemingly unrelated regression (SURE) estimate of $\beta_{i}$ is

$$
\hat{y}_{i, T+1}^{g}=x_{i, T+1}^{t} \hat{\beta}_{i}^{g}
$$

with $\hat{\beta}_{i}$ being the $i$-th subvector of the generalized least squares estimate of $\beta_{a}$ in (3.3), $\hat{\beta}_{a}=\left(X^{* \prime} \hat{\Omega}^{-1} X^{*}\right)^{-1} X^{* \prime} \hat{\Omega}^{-1} y$, with $\hat{\Omega}$ being a consistent estimate of $\Omega$.

4. The forecast based on a pooled feasible generalized least squares estimate of $\beta_{i}$

$$
{\stackrel{\wedge}{y_{i, T+1}^{p g}}}^{p g} x_{i, T+1}^{\prime} \stackrel{\wedge}{\beta}^{p g}
$$

with $\hat{\beta}^{p g}=\left(X^{\prime} \hat{\Omega}^{-1} X\right)^{-1} X^{\prime} \hat{\Omega}^{-1} y$.

The predictors (3.4)-(3.5) have been used by Garcia-Ferrer et al. (1987) and Zellner and Hong (1989) to obtain point forecasts of GNP growth rates, and by Zellner et al. (1991) to forecast turning points in GNP growth. They have also studied shrinkage forecasts which are linear combinations of individual and pooled forecasts. We are interested in the behavior of these predictors when the time-dimension of the sample becomes large. We compare the individual forecasts with the pooled forecasts when $T$ grows while $N$ remains fixed and investigate the conditions under which pooling leads to improved forecast performance.

To compare the forecast performance, we use the mean square forecast error (MSFE) criterion

$$
\begin{aligned}
\operatorname{MSFE}\left(\hat{y}_{i, T+1}\right)= & E\left\{x _ { i , T + 1 } ^ { \prime } \left[\left(\tilde{\beta}_{i}-\tilde{\beta}_{i}^{*}\right)\left(\tilde{\beta}_{i}-\tilde{\beta}_{i}^{*}\right)^{\prime}+\left(\beta_{i}-\tilde{\beta}_{i}^{*}\right)\left(\beta_{i}-\tilde{\beta}_{i}^{*}\right)^{\prime}\right.\right. \\
& \left.\left.+2\left(\beta_{i}-\tilde{\beta}_{i}\right)\left(\tilde{\beta}_{i}-\tilde{\beta}_{i}^{*}\right)^{\prime}\right] x_{i, T+1}\right\}+\sigma_{i}^{2},
\end{aligned}
$$

where $\tilde{\beta}_{i}$ denotes an estimator of $\beta_{i}$ (one of the estimators $(3.4)-(3.7)$ ), $\tilde{\beta}_{i}^{*}=$ plim $\tilde{\beta}_{i}$ and $\sigma_{i}^{2}$ is the $i$-th diagonal element of $\Sigma$. The cross-term $x_{i, T+1}^{\prime}\left(\beta_{i}-\tilde{\beta}_{i}\right) \varepsilon_{i, T+1}$ has expectation 
zero and therefore does not appear in (3.8). In large samples, the third term between brackets in (3.8) converges to zero and the value of the MSFE will be determined by the variance and the squared bias of the parameter estimator $\tilde{\beta}_{i}$ and the disturbance variance $\sigma_{i}^{2}$. Comparing the MSFE of various forecasts is basically reduced to comparing the MSE's of the estimators used to compute the forecasts.

The results obtained by McElroy (1977) can be applied to compare the GLS estimator $\hat{\beta}_{p}^{g}=\left(\hat{\beta}_{1}^{g r}, \ldots, \hat{\beta}_{N}\right)^{\prime}$ in $(3.6)$ for $\beta_{a}$ with the pooled GLS estimator in $(3.8) \hat{\beta}_{a}^{p g}=\left(\iota_{N} \otimes\right.$ $\left.\hat{\beta}^{\prime}\right)$, where $\iota_{N}$ is a $N \times 1$ vector of ones.

When $\Omega$ is unknown, a consistent estimate can be used and the results by McElroy (1977) hold for a large sample. When $\Omega=\sigma^{2} I_{n}$, McElroy's (1977), result specializes accordingly and can be used to compare the OLS estimator $\hat{\beta}_{a}=\left(\hat{\beta}_{1}^{\prime}, \ldots, \hat{\beta}_{N}^{\prime}\right)^{\prime}$ in (3.4) with the pooled estimator $\hat{\beta}_{a}^{p}$ in (3.5).

When $\Omega$ is known the restricted GLS estimator $\hat{\beta}_{a}^{p g}$ is better than $\hat{\beta}_{a}^{g}$ by the strong MSE criterion defined by McElroy if for all $N k \times 1$ vectors $\ell \neq 0$,

$$
\ell^{\prime}\left[M S E\left(\sqrt{n} \hat{\beta}_{a}^{g}\right)-M S E\left(\sqrt{n} \hat{\beta}_{a}^{p g}\right)\right] \ell \geq 0 .
$$

Notice that the condition (3.9) implies superiority of the pooled estimator for each country.

Imposing restrictions which do not hold true introduces a bias in the estimator. When the condition (3.9) holds the size of the bias is outweighed by the reduction of the variance of the estimator.

Using results in McElroy, expression (3.9) can be shown to hold iff $\lambda_{n} \leq \frac{1}{2}$ with $\lambda_{n}=\delta_{n}^{\prime}\left(R V_{n} R^{\prime}\right)^{-1} \delta_{n} / 2$ and $\delta_{n}=\sqrt{n}\left(R \beta_{a}\right)$. Here $R \beta_{a}=0$ denotes the restrictions on $\beta_{a}$ when we pool across countries,

$$
R=\left[\begin{array}{ccccc}
I_{k} & -I_{k} & 0 & \cdots & 0 \\
I_{k} & 0 & -I_{k} & & 0 \\
I_{k} & & & & -I_{k}
\end{array}\right] \text { is a } q \times N k \text { matrix, with } q=(N-1) k,
$$

and $V_{n}=n\left(X^{* /} \Omega^{-1} X^{*}\right)^{-1}$ is the covariance matrix of $\sqrt{n} \hat{\beta}_{a}^{g}$. The null hypothesis $R \beta_{a}=0$ is true iff $\lambda_{n}=0$. The restricted estimator $\hat{\beta}_{a}^{p g}$ is better than $\hat{\beta}_{a}^{g}$ by the first weak MSE criterion iff the trace of the difference of the MSE matrices in (3.9) is non-negative, that is whenever,

$$
\lambda_{n} \leq \frac{1}{2} \mu_{n} \operatorname{tr}\left[V_{n} R^{\prime}\left(R V_{n} R^{\prime \prime}\right)^{-1} R V_{n}\right]=\theta_{n}
$$

with $\mu_{n}$ being the smallest characteristic root of $V_{n}^{-1}$.

Finally, the pooled GLS estimator is better than the GLS estimator by the second weak MSE criterion defined by McElroy (1977) as

$$
E\left[n\left(\hat{\beta}_{a}^{\wedge g}-\beta_{a}\right)^{\prime} V_{n}^{-1}\left(\hat{\beta}_{a}^{g}-\beta_{a}\right)-n\left(\hat{\beta}_{a}^{p g}-\beta_{a}\right)^{\prime} V_{n}^{-1}\left(\hat{\beta}_{a}^{p g}-\beta_{a}\right)\right] \geq 0
$$


which holds iff $\lambda_{n} \leq q / 2$, i.e. iff $2 \lambda_{n}$ is smaller than the number of restrictions on regression coefficients in the system.

As shown by McElroy (1977) when the $X_{i}$ 's are strictly exogenous (standard regression model) and $\Omega$ is known or consistently estimated, the test statistic

$$
F^{*}(\Omega)=\frac{n\left(R \hat{\beta}_{a}^{g}\right)^{\prime}\left(R V_{n} R^{\prime}\right)^{-1}\left(R \hat{\beta}_{a}^{g}\right) / q}{S S E\left(\beta_{a}^{g}\right) /(n-q)} \sim F\left(q, n-q, \lambda_{T}\right)
$$

with $S S E\left(\hat{\beta}_{a}^{g}\right)=\left(y-X^{*} \hat{\beta}_{a}^{g}\right)^{\prime} \Omega^{-1}\left(y-X^{*} \hat{\beta}_{a}^{g}\right)$ has a noncentral $F$-distribution. It can be used to test hypotheses about $\lambda$. Rejecting the null hypothesis when the statistic is large provides a uniformly most powerful test for $\lambda_{n} \leq \lambda^{*}$ against $\lambda_{n}>\lambda^{*}$ where $\lambda^{*}=0, \frac{1}{2}, \theta_{n}$ or $q / 2$ depending upon the chosen MSE criterion.

As $n \rightarrow \infty$ (e.g. for $T \rightarrow \infty$ and fixed $N), q F^{*}$ in (3.13) converges to a (non)-central $\chi^{2}(q, \lambda)$ distribution with $\lambda=\lim _{n \rightarrow \infty} \lambda_{n}$, provided the sequence of alternative hypotheses is chosen in such a way that $\lambda_{n}$ converges to a finite limit $\lambda$. When $\Omega$ is replaced by a consistent estimator and/or in the presence of predetermined variables among the regressors, the same limiting distribution for $q F^{* *}$ results. Also, as $n \rightarrow \infty$ the covariance matrices for both $\sqrt{n} \hat{\beta}_{a}$ and $\sqrt{n} \hat{\beta}_{a}^{p \cdot g}$ converge to constant matrices. If the restrictions $R \beta_{a}=0$ do not hold, $\delta_{n}$ and hence the bias of $\sqrt{n} \ddot{\beta}_{a}^{p Q}$ increase without bound while the unrestricted estimator $\sqrt{n} \stackrel{\beta}{\beta}_{a}$ remains unbiased. Therefore, in case $\underset{\wedge p g}{R \beta_{a}} \neq 0$, for each of the three MSE criteria, there exists a sufficiently large $n$ to make $\hat{\beta}_{a}^{p g}$ worse than $\hat{\beta}_{a}^{g}$ in terms of MSE. In the case where the restrictions are false, the non-centrality parameter $\lambda_{n}$ increases without bound. As a result, the power of a test of $H_{0}: \lambda_{n} \leq \lambda^{*}$ tends to one and the test is consistent.

In the next section, we shall report the findings of an empirical analysis of the MSFE based on unrestricted, pooled and shrunken estimators. In particular, we shall investigate under which conditions and for which sample size it pays to use a pooled or a shrinkage estimator instead of an unrestricted estimator.

\subsection{Empirical analyses}

In this section we investigate an AR(3) model and an AR(3) model with leading indicators, denoted AR(3)LI hereafter, used by Garcia-Ferrer et al. (1987) and Zellner and Hong (1989). The data set consists of the post World War II real GDP growth rates of 18 OECD countries. The data for the inidividual countries have starting dates varying between 1949 and 1957 and end in 1990 (see appendix A). In section 3.3.1, we present the models, estimate and test them. Section 3.3 .2 is devoted to a simulation study of the finite sample properties of McElroy's criteria for pooling. The empirical models for a subset of the 18 OECD countries are used in the simulations. Finally, in subsection 3.3.3 we check the pooling restrictions for the 18 countries using McElroy's criteria. 


\subsubsection{The models}

As in Garcia-Ferrer et al. (1987) and Zellner and Hong (1989), the following AR(3)LI model was employed to generate one-year-ahead forecasts of the growth rate of real GDP, but now for the period 1981-1990, for eighteen countries:

$$
\begin{aligned}
& y_{i, t}=\beta_{0 i}+\beta_{1 i} y_{i, t-1}+\beta_{2 i} y_{i, t-2}+\beta_{3 i} y_{i, t-3} \\
& +\beta_{4 i} S R_{i, t-1}+\beta_{5 i} S R_{i, t-2}+\beta_{6 i} G M_{i, t-1}+\beta_{7 i} W R_{t-1}+\varepsilon_{i, t},
\end{aligned}
$$

where

$$
\begin{aligned}
y_{i, t} & =\text { first difference of the logarithm of real output } \\
S R_{i, t} & =\text { frst difference of the log of a stock price index divided by a } \\
& \text { general price index } \\
G M_{i, t}= & \text { first difference of the log of the nominal money supply divided } \\
& \text { by a general price index } \\
W R_{i}=\text { world return = median of countries" real stock return in period t } & =\text { parameters for country } i \\
\beta_{i j}=\text { disturbance term. } &
\end{aligned}
$$

The AR(3) model arises as a special case of model (3.14) when $\beta_{4 i}=\beta_{5 i}=\beta_{6 i}=\beta_{7 i}=0$. We use the AR(3) as the benchmark model to evaluate the leading indicators.

To illustrate the gains from pooling when $T$ is small, we performed the actual onestep-ahead forecasting procedure as in Garcia-Ferrer et al. (1987) and Zellner and Hong (1989). We started forecasting the period 1983-1990 using all data (columns two and three of Table 3.1 ); next we excluded the first 5 annual observations for each country when estimating the parameters of the AR(3) model (columns four and five of Table 3.1); then we excluded the first 10 observations (columns six and seven); and finally we excluded the first 15 observations for each country (columns seven and eight).

As shown in section 2 of this chapter we expect to see, as the number of used observations $T$ gets smaller, that the forecasting performance of the pooled forecast based on $\hat{\beta}_{i}^{p}$ dominates the individual predictor. This becomes apparent in two ways in Table 3.1 . Firstly, the number of countries for which the individual forecast is better declines as $T$ becomes smaller. Secondly, the difference in median RMSE's increases as $T$ decreases.

In Tables 3.2 and 3.3 we compare the pooled and individual forecasting performance of the AR(3) and AR(3)LI models for different periods of out of sample forecasts. For every forecast the data till the date to be forecasted are used. For every forecast the system is reestimated and a one step ahead, out of sample, forecast is made.

Tables 3.2 and 3.3 report RMSE's for forecasts based on country-specific OLS parameter estimates and pooled parameter estimates for the forecasting periods 1974-87, 1974-90 and 1983-90. For the three forecast periods and for both models, the pooled forecasts dominate the individual forecasts in at least 14 out of 18 the cases. Also the median of the Root Mean Square Forecast Errors (RMSFEs) is lowest for the pooled forecasts in the six cases. This finding is in line with those of Garcia-Ferrer et al. (1987) for a forecast period 1974-1981 and Zellner and Hong (1989) for the periods 1974-1981 and 1974-1984. The data for the period 1982-1990 exhibit much less movement than the observations in the estimation period. Notice also that for the forecast period 1983-1990, surprisingly the 


\begin{tabular}{|c|c|c|c|c|c|c|c|c|}
\hline \multirow[b]{3}{*}{ FORECAST BASED ON } & \multicolumn{8}{|c|}{$\begin{array}{c}\text { NUMBER OF OBSERVATIONS } \\
\text { EXCLUDED }\end{array}$} \\
\hline & \multicolumn{2}{|c|}{0} & \multicolumn{2}{|c|}{5} & \multicolumn{2}{|c|}{10} & \multicolumn{2}{|c|}{15} \\
\hline & $\hat{\beta}_{i}$ & $\hat{\beta}_{i}^{p}$ & $\hat{\hat{\beta}_{i}}$ & $\hat{\beta}_{i}^{p}$ & $\hat{\beta_{i}}$ & $\hat{\beta}_{i}^{F}$ & $\hat{\hat{\beta}_{i}}$ & $\hat{\beta_{i}}$ \\
\hline Australia & 3.66 & 3.36 & 3.66 & 3.41 & 3.60 & 3.35 & 3.26 & 3.30 \\
\hline Austria & 1.53 & 0.92 & 1.30 & 0.89 & 0.92 & 0.74 & 1.03 & 0.75 \\
\hline Bellgium & 1.38 & 1.79 & 1.54 & 1.71 & 1.59 & 1.69 & 1.97 & 1.74 \\
\hline Canada & 3.90 & 3.47 & 4.26 & 3.44 & 4.39 & 3.43 & 4.59 & 3.35 \\
\hline Denmark & 1.86 & 1.81 & 2.04 & 1.77 & 2.45 & 1.81 & 2.66 & 1.72 \\
\hline Finland & 1.49 & 1.37 & 1.29 & 1.38 & \begin{tabular}{|l|}
1.23 \\
\end{tabular} & 1.52 & 1.26 & 1.69 \\
\hline France & 1.44 & 1.59 & 1.67 & 1.52 & 1.69 & 1.47 & 1.70 & 1.46 \\
\hline Germany & 4.27 & 4.50 & 4.40 & 4.30 & 4.47 & 4.39 & 4.37 & 4.34 \\
\hline Ireland & 2.23 & 1.68 & 2.48 & 1.67 & 3.78 & 1.64 & 3.95 & 1.74 \\
\hline Italy & 2.40 & 1.55 & 2.39 & 1.47 & 2.42 & 1.52 & 2.43 & 1.52 \\
\hline Japan & 1.09 & 0.99 & 1.05 & 0.98 & 1.14 & 1.06 & 1.32 & 1.23 \\
\hline Netherlands & 1.67 & 1.46 & 1.60 & 1.42 & 1.42 & 1.26 & 1.61 & 1.27 \\
\hline Norway & 4.95 & 3.98 & 5.00 & 3.91 & 5.52 & 3.86 & 5.66 & 3.71 \\
\hline Spain & 1.03 & 1.19 & 2.03 & 1.22 & 2.00 & 1.34 & 1.79 & 1.54 \\
\hline Sweden & 1.41 & 1.19 & 1.47 & 1.16 & 1.72 & 1.20 & 1.69 & 1.22 \\
\hline Switzerland & 1.54 & 1.48 & 1.56 & 1.48 & 1.65 & 1.40 & 2.00 & 1.43 \\
\hline UK & 2.38 & 1.90 & 2.51 & 1.88 & 2.77 & 1.93 & 2.90 & 1.96 \\
\hline USA & 3.15 & 2.74 & 3.59 & 2.64 & 4.20 & 2.76 & 4.13 & 2.75 \\
\hline median & 1.76 & 1.63 & 2.04 & 1.59 & 2.21 & 1.58 & 2.22 & 1.71 \\
\hline
\end{tabular}

Table 3.1: The root mean square forecast error for the individual country forecast and for the pooled forecast using the $\operatorname{AR}(3)$ model when up to 15 observations are excluded from the estimation period. 


\begin{tabular}{|l|l|l|l|l|l|l|}
\hline \multirow{2}{*}{$\begin{array}{l}\text { Period } \\
\text { gamma }\end{array}$} & \multicolumn{5}{|c|}{ AR(3) } \\
\cline { 2 - 7 } & unpooled & pooled & unpooled & pooled & unpooled & pooled \\
\hline Australia & 4.73 & 3.21 & 4.34 & 2.92 & 3.66 & 3.36 \\
\hline Austria & 3.30 & 2.60 & 3.02 & 2.36 & 1.53 & 0.92 \\
\hline Belgium & 3.19 & 3.24 & 2.95 & 2.98 & 1.38 & 1.79 \\
\hline Canada & 3.78 & 3.37 & 3.48 & 3.12 & 3.90 & 3.47 \\
\hline Denmark & 3.50 & 3.40 & 3.36 & 3.30 & 1.86 & 1.81 \\
\hline Finland & 3.63 & 3.34 & 3.34 & 3.12 & 1.49 & 1.37 \\
\hline France & 3.15 & 2.42 & 2.91 & 2.30 & 1.44 & 1.59 \\
\hline Germany & 5.05 & 4.67 & 4.62 & 4.36 & 4.27 & 4.50 \\
\hline Ireland & 4.81 & 4.36 & 4.45 & 4.02 & 2.23 & 1.68 \\
\hline Italy & 3.85 & 3.23 & 3.56 & 3.00 & 2.40 & 1.55 \\
\hline Japan & 4.36 & 2.98 & 3.98 & 2.73 & 1.09 & 0.98 \\
\hline Netherlands & 3.39 & 2.58 & 3.13 & 2.41 & 1.67 & 1.45 \\
\hline Norway & 2.85 & 3.02 & 3.70 & 3.15 & 4.94 & 3.97 \\
\hline Spain & 3.00 & 2.57 & 2.74 & 2.40 & 1.03 & 1.19 \\
\hline Sweden & 2.91 & 2.76 & 2.66 & 2.52 & 1.41 & 1.18 \\
\hline Switzerland & 3.73 & 3.77 & 3.46 & 3.43 & 1.54 & 1.48 \\
\hline UK & 3.49 & 3.27 & 3.36 & 3.10 & 2.38 & 1.90 \\
\hline USA & 4.01 & 3.79 & 3.66 & 3.50 & 3.15 & 2.74 \\
\hline median & 3.57 & 3.24 & 3.41 & 3.05 & 1.76 & 1.63 \\
\hline
\end{tabular}

Table 3.2: Eighteen countries RMSFEs of one-year-ahead forecasts, 1974-1987, 1974-1990 and 1983-1990. 


\begin{tabular}{|c|c|c|c|c|c|c|}
\hline \multirow{3}{*}{$\begin{array}{l}\text { Period } \\
\text { gamma }\end{array}$} & \multicolumn{6}{|c|}{ AR(3)LI } \\
\hline & \multicolumn{2}{|c|}{$1974-87$} & \multicolumn{2}{|c|}{197490} & \multicolumn{2}{|c|}{$1983-90$} \\
\hline & unpooled & pooled & unpooled & pooled & unpooled & pooled \\
\hline Australia & 4.94 & 2.63 & 4.77 & 2.42 & 3.55 & 1.91 \\
\hline Austria & 3.42 & 2.51 & 3.15 & 2.29 & 3.02 & 1.88 \\
\hline Belgium & 1.87 & 2.41 & 2.31 & 2.38 & 2.93 & 2.21 \\
\hline Canada & 3.99 & 2.91 & 3.65 & 2.67 & 4.08 & 3.48 \\
\hline Denmark & 4.00 & 3.92 & 4.02 & 3.94 & 3.73 & 3.06 \\
\hline Finland & 3.74 & 2.92 & 3.61 & 2.73 & 2.66 & 1.53 \\
\hline France & 3.13 & 2.72 & 2.90 & 2.68 & 1.51 & 2.30 \\
\hline Germany & 4.19 & 3.98 & 3.90 & 3.75 & 3.90 & 3.69 \\
\hline Ireland & 4.48 & 4.12 & 4.25 & 3.88 & 2.60 & 1.78 \\
\hline Italy & 3.66 & 2.42 & 3.54 & 2.46 & 3.47 & 1.85 \\
\hline Japan & 4.17 & 2.77 & 3.95 & 2.58 & 2.45 & 1.23 \\
\hline Netherlands & 3.23 & 2.47 & 4.00 & 2.46 & 2.90 & 2.46 \\
\hline Norway & 3.37 & 3.12 & 4.20 & 3.88 & 5.36 & 5.43 \\
\hline Spain & 3.03 & 2.28 & 2.95 & 2.09 & 2.32 & 1.24 \\
\hline Swreden & 2.90 & 2.48 & 2.65 & 2.34 & 2.58 & 1.75 \\
\hline Switzerland & 3.89 & 3.46 & 3.71 & 3.19 & 2.51 & 2.13 \\
\hline $\mathrm{UK}$ & 2.83 & 2.64 & 4.48 & 3.35 & 5.64 & 3.76 \\
\hline USA & 3.50 & 3.42 & 3.49 & 3.30 & 3.75 & 2.62 \\
\hline median & 3.58 & 2.74 & 3.63 & 2.68 & 2.79 & 2.17 \\
\hline
\end{tabular}

Table 3.3: Eighteen countries 'RMSFEs of one-year-ahead forecasts, 1974-1987, 1974-1990 and 1983-1990. 
AR(3) models performs better than the AR(3)LI model in terms of RMSFE. For the forecast period 1974-1987, the AR(3)LI clearly performs better than the AR(3) model in most instances. There are small differences with results reported by Min and Zellner (1993) for the same forecast period. These are due to the fact that they use GNP and GDP data from the period 1954-1973 with data for 1951-53 serving as initial values whereas in the present study strictly GDP data are used for all countries from the varying start dates as listed in Appendix A.

In the previous results we did not take into account possible correlations between countries. The ignorance of possible correlatins can influence the forecasting performance as it can lead to efficiency loss when estimating the parameters. We therefore examine the presence of contemporaneous correlation between the disturbances for the 18 countries in the AR(3) and AR(3)LI models respectively.

\begin{tabular}{|c|c|c|c|}
\hline Hypothese: $\bar{H}_{0}$ & E: diagonal & $\Sigma$ : diagonal & $\Sigma:$ block-diagonal \\
\hline Hypothese: $H_{1}$ & $\Sigma:$ unrestricted & $\Sigma:$ block-diagonal $^{(*)}$ & $\bar{\Sigma}:$ unrestricted \\
\hline Models & & & \\
\hline $\begin{array}{l}\text { AR(3)LI } \\
1961-80\end{array}$ & $\begin{array}{c}167.68 \\
d f=153 \\
p=.197\end{array}$ & $\begin{array}{c}99.40 \\
\mathrm{df}=17 \\
\mathrm{p}<.001\end{array}$ & $\begin{array}{c}77.28 \\
\mathrm{df}=136 \\
\mathrm{p}>.5\end{array}$ \\
\hline $\begin{array}{l}\text { AR(3)LI } \\
1961-90\end{array}$ & $\begin{array}{c}290.88 \\
\mathrm{df}=153 \\
\mathrm{p}<.01\end{array}$ & $\begin{array}{c}149.67 \\
\mathrm{df}=17 \\
\mathrm{p}<.001\end{array}$ & $\begin{array}{c}141.21 \\
\mathrm{df}=136 \\
\mathrm{p}=.362\end{array}$ \\
\hline $\begin{array}{l}\text { AR(3) } \\
1961-80\end{array}$ & $\begin{array}{c}353.46 \\
\mathrm{df}=153 \\
\mathrm{p}<.001\end{array}$ & $\begin{array}{c}105.94 \\
\mathrm{df}=17 \\
\mathrm{p}<.001\end{array}$ & $\begin{array}{c}247.5 \\
\mathrm{df}=136 \\
\mathrm{p}<.001\end{array}$ \\
\hline $\begin{array}{l}\text { AR(3) } \\
1961-90\end{array}$ & $\begin{array}{c}474.67 \\
d f=153 \\
p<.001\end{array}$ & $\begin{array}{c}137.79 \\
\mathrm{df}=17 \\
\mathrm{p}<.001\end{array}$ & $\begin{array}{c}336.88 \\
\mathrm{df}=136 \\
\mathrm{p}<.01\end{array}$ \\
\hline
\end{tabular}

(*) We distinguish the following 7 blocks: (1) Canada, USA; (2) Australia, Japan; (3) Denmark, Finland, Norway, Sweden; (4) Belgium, France, Germany, Netherlands; (5) UK, Ireland; (6) Austria, Switzerland; (7) Italy, Spain.

Table 3.4: Testing for contemporaneous error covariances.

For the estimation period 1961-1980, as could be expected, the residuals of the AR(3) model are more strongly contemporaneously correlated than the residuals of the AR(3)LI model. Including leading indicators which are approximately white noise accounts for a major part of the contemporaneous residual correlation present in the $\operatorname{AR}(3)$ model. From the estimated residual correlations most of which are positive, it also appears that the countries can be clustered in regional groups exhibiting much within group contemporaneous residual correlation and little between group contemporaneous residual correlation 
indicating that shocks to real GDP growth are partly synchronized within blocks and uncorrelated between blocks. We distinguish the following seven regional blocks : (1) Canada, USA; (2) Australia, Japan; (3) Denmark, Finland, Norway, Sweden; (4) Belgium, France, Germany, Netherlands; (5) Ireland, UK; (6) Austria, Switzerland; (7) Italy, Spain.

To formally test for contemporaneous residual correlation, we use an LM statistic proposed by Breusch and Pagan (1980) for testing the null hypothesis of a diagonal $\Sigma$. Under $H_{0}$, the Lagrange multiplier statististic $L M=T \Sigma_{i=2}^{N} \Sigma_{j=1}^{i-1} r_{i j}^{2}$ with $r_{i j}$ being the sample correlation coefficients between the residuals of the OLS estimates for countries $i$ and $j$, has an asymptotic $\chi^{2}[N(N-1) / 2]$ distribution. The results for the LM test are given in Table 3.4 .

The $\chi^{2}$-statistics reported in Table 3.4 indicate that the diagonality of $\Sigma$ is not rejected for the AR(3)LI model for the observation period 1961-1980 when tested against an unrestricted $\Sigma$ matrix. When tested against a block diagonal matrix, diagonality has to be rejected. For the longer period 1961-1990, we reject the null hypothesis of a diagonal $\Sigma$ matrix. This is possibly due to structural changes that occurred in the 1980 s. For this latter period, the block-diagonal structure is not rejected for the AR(3)LI model. For the AR(3) model the null hypothesis has to be rejected in all instances. This is not surprising in view of the finding by Garcia et al. (1987) that including the common leading indicator $W R_{t-1}$ which is approximately white noise accounts for interdependencies among the white noise disturbances of the countries.

Note that Chib and Greenberg (1995) report that when a time-varying parameter version of the $\mathrm{AR}(3) \mathrm{LI}$ model is employed for five countries (Australia, Canada, Germany and the U.S.) the matrix $\Sigma$ is found to be diagonal.

\subsubsection{Properties of pooling restriction tests}

Before we check the appropriateness of pooling in a system of 18 equations, we investigate the small sample properties of the $F$-statistic given in (3.13) for testing the pooling restriction $H_{0}: R \beta_{a}=0$ against $H_{1}: R \beta_{a} \neq 0$ and of McElroy"s strong and weak pooling criteria. The simulation results have been obtained using models for the following sets of countries: Belgium, Germany, France and the Netherlands and Canada and the US. Observe that we identified in the previous sections these countries as belonging to two blocks of correlated countries. The model consists of a set of six third order autoregressions with two leading indicators

$$
\underset{(6 \times 6)(6 \times 1)}{\Phi(L)} y_{t}=\underset{(6 \times 6)(6 \times 1)}{B} x_{1, t}+\underset{(6 \times 1)}{\gamma} x_{2, t}+\underset{(6 \times 1)}{\varepsilon_{t}}
$$

where $\Phi(L)$ is a diagonal lag polynomial matrix with third degree polynomial on the main diagonal,$B$ and $\gamma$ denote respectively a matrix and a vector of coefficients. The leading indicators $x_{1 t}$ and $x_{2 t}$ and the disturbance vector $\varepsilon_{t}$ satisfy the following properties

$$
x_{1, t} \sim \operatorname{IIN}\left(0, \Sigma_{1}\right), x_{2, t} \sim \operatorname{IIN}\left(0, \sigma_{2}^{2}\right), \varepsilon_{t} \sim \operatorname{IIN}\left(0, \Sigma_{3}\right)
$$

with $\Sigma_{1}$ being diagonal and $\Sigma_{3}$ being a block-diagonal covariance matrix. The variables $x_{1, t}, x_{2, t}$ and $\varepsilon_{t}$ are mutually independent. The vector $x_{1, t}$ can be interpreted as a country 


\begin{tabular}{|l|l|l|l|l|}
\hline Model & $\Sigma$ & $F$-value & $p$-value & $p$-value \\
\hline & & & $\lambda=0$ & $\lambda=q / 2$ \\
\hline AR(3) & $\sigma^{2} I$ & 0.5763 & 0.9244 & 0.9944 \\
\hline AR(3) & Full & 1.0532 & 0.4032 & 0.8839 \\
\hline AR(3)LI & $\sigma^{2} I$ & 1.2580 & 0.2156 & 0.6772 \\
\hline AR(3)LI & Block & 1.1099 & 0.3320 & 0.8453 \\
\hline
\end{tabular}

Table 3.5: Testing for pooling for a subset of 6 countries (Canada, USA, Belgium, France, Germany, Netherlands).

specific leading indicator. The variable $x_{2, t}$ can be interpreted as a common leading indicator (e.g. $W R_{t-1}$ ). The block-diagonal structure of $\Sigma_{3}$ reflects the finding that the disturbances within European and North-American subgroups are correlated and that the between-subgroup correlations are zero. The model (3.15)-(3.16) implies a third order VAR model for $y t$ with a diagonal VAR-matrix and a full disturbance covariance matrix $\Sigma=B \Sigma_{1} B^{\prime}+\sigma_{2}^{2} \gamma \gamma^{\prime}+\Sigma_{3}$. The implied error-component structure of the disturbance term. of the AR(3) model will be ignored in the sequel.

Both the AR(3)LI model (3.15) and the implied VAR(3) model have been simulated. The models have been simulated under parameter heterogeneity across countries and under parameter homogeneity. The parameter values used in the simulations for the heterogenous AR(3)LI model are set equal to the OLS estimates of model (3.15) taking $x_{1, t}$ to be the vector of observed $G M_{i, t-1}$ and $x_{2, t}$ to be equal to $W R_{t-1}$. Under parameter homogeneity, OLS estimates of the equation in (3.15) for the US are used for all six countries. In a similar way estimates of the $\mathrm{AR}(3)$ model under respectively parameter heterogeneity and homogeneity have been obtained. On the basis of the data for the six countries, parameter homogeneity is not rejected for model (3.14) when testing regression parameter equality across countries using an F-test. Obviously, McElroy's criteria do not reject pooling either. The details for these tests are given in Table 3.5.

In figures 3.1 and 3.2 , the empirical distributions of the $F$-statistics in (3.13) obtained by simulation are given. The number of runs is 1000 . Rejection frequencies when the test-statistic is compared with the critical value of respectively a central $F$-distribution with $q$ and $n-q$ degrees of freedom and that of a nom-central $F\left(q, n-q, \lambda_{T}\right)$ with $\lambda_{T}=q / 2$ are reported in Table 3.6. A nominal significance level of $5 \%$ is used.

Under parameter homogeneity, the rejection frequencies are very small when McElroy"s second weak criterion is tested. With the exception of the AR(3) model with unrestricted disturbance covariance matrix, the rejection frequencies when a central $F$-distribution is used are also substantially smaller than $5 \%$ for the model under parameter homogeneity. An $F$-test appears to be too conservative whether the correct disturbance covariance is assumed or not.

Under parameter heterogeneity, as expected, the power of both tests is found to increase as the sample size increases. For the test of parameter equality $\lambda=0$, the rejection frequency for values of $T$ equal or larger than 50 , is found to be fairly large (larger than $60 \%$ ).

For $T=25$, McElroy's second weak criterion rejects rather infrequently the incorrect parameter restrictions. As $T$ increases, the gain resulting from trading-off some bias 

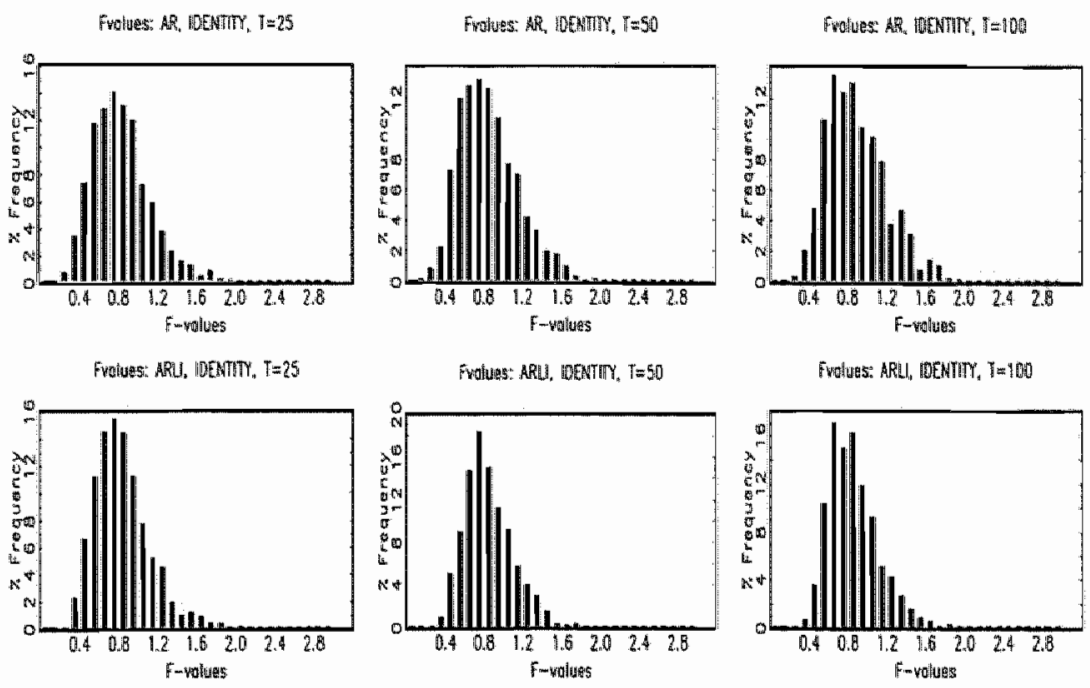

Fvolues: AR, FULL $t=25$

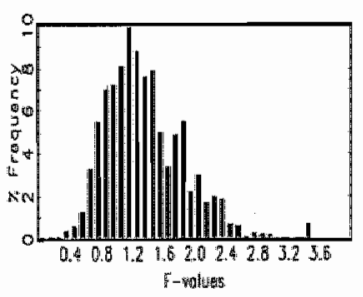

Fvelues: AR, FULL, T=50

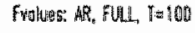
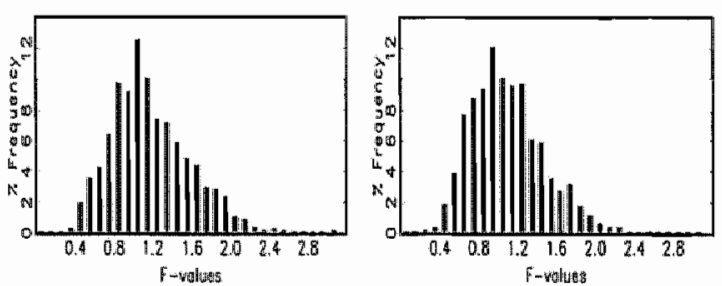

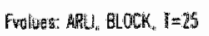

Frolues: RRI, HLOCK, I $=50$

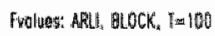
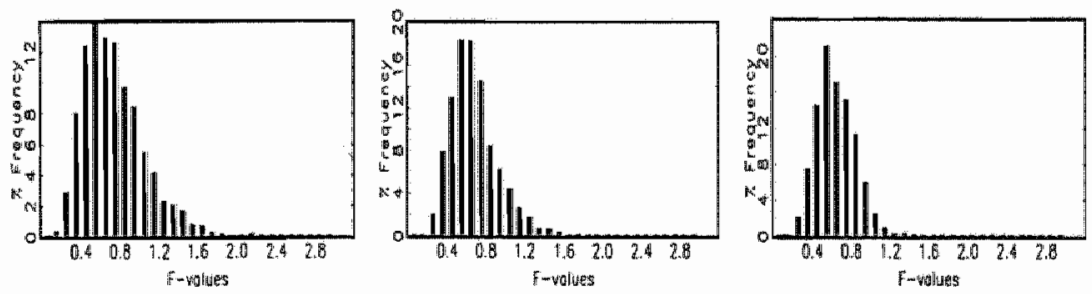

Figure 3.1: Empirical distribution of the simulated F-statistic (3.13) under parameter homogeneity. 


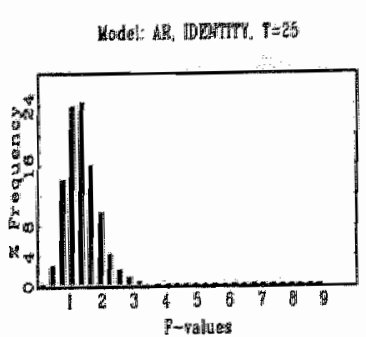

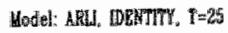

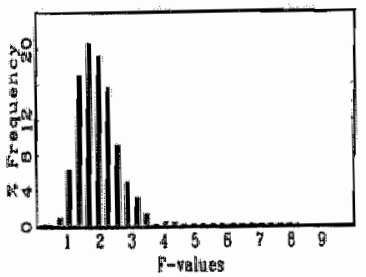

Haded; AR FU:L, I:=25

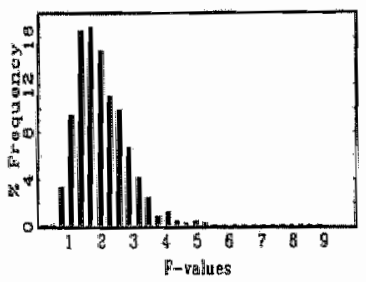

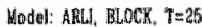

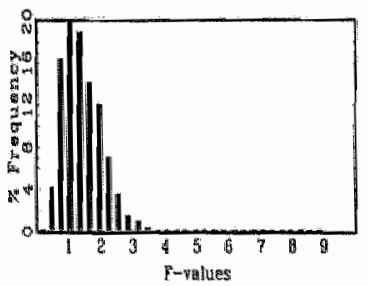

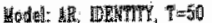

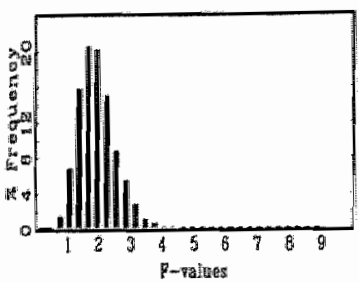

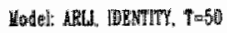

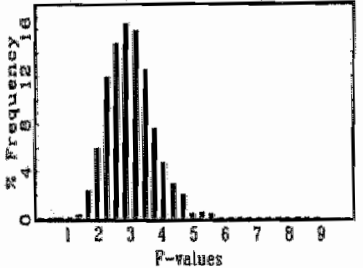

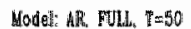

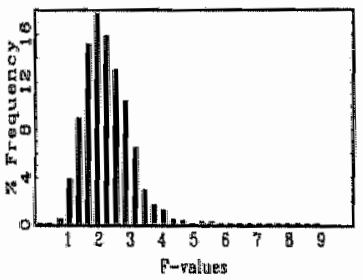

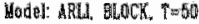

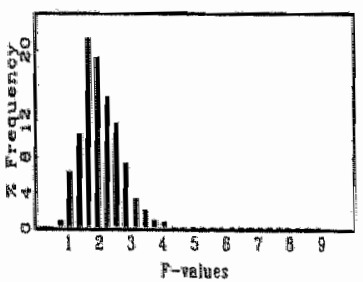

Ycod: AR, DERTT, $T=1 \mathrm{AO}$

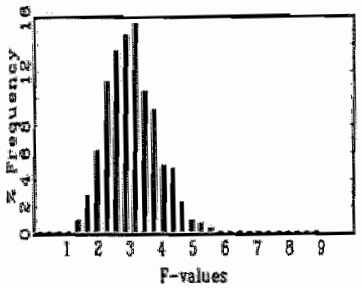

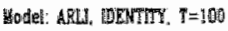

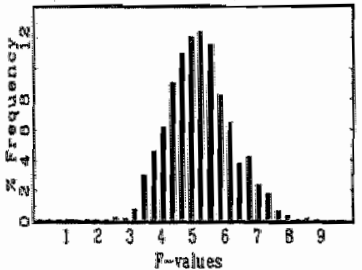

Wodel: $A R$ PUL, $I=100$

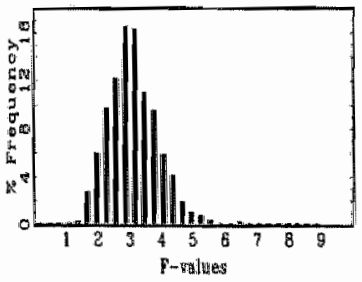

Hodel. ARL, BLCCK $T=100$

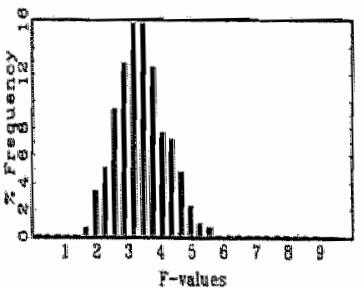

Figure 3.2: Empirical distribution of the simulated F-statistic (3.13) under parameter heterogeneity. 


\begin{tabular}{|l|l|l|l|l|l|l|}
\hline \multicolumn{2}{|c|}{} & & \multicolumn{2}{l|}{ Homogenous } & \multicolumn{2}{l|}{ Heterogenous } \\
\hline T & estimator & $\Sigma$ & $\lambda=0$ & $\lambda=q / 2$ & $\lambda=0$ & $\lambda=q / 2$ \\
\hline 25 & AR(3)LI & $\sigma^{2} I$ & 0.024 & 0.000 & 0.722 & 0.251 \\
\hline 50 & AR(3)LI & $\sigma^{2} I$ & 0.010 & 0.000 & 0.996 & 0.871 \\
\hline 100 & $\mathrm{AR}(3) \mathrm{LI}$ & $\sigma^{2} I$ & 0.023 & 0.000 & 1.000 & 1.000 \\
\hline 25 & $\mathrm{AR}(3)$ & $\sigma^{2} I$ & 0.017 & 0.000 & 0.270 & 0.038 \\
\hline 50 & $\mathrm{AR}(3)$ & $\sigma^{2} I$ & 0.019 & 0.001 & 0.672 & 0.207 \\
\hline 100 & $\mathrm{AR}(3)$ & $\sigma^{2} I$ & 0.032 & 0.000 & 0.984 & 0.810 \\
\hline 25 & $\mathrm{AR}(3) \mathrm{LI}$ & Block & 0.019 & 0.000 & 0.369 & 0.095 \\
\hline 50 & $\mathrm{AR}(3) \mathrm{LI}$ & Block & 0.007 & 0.000 & 0.821 & 0.354 \\
\hline 100 & $\mathrm{AR}(3) \mathrm{LI}$ & Block & 0.005 & 0.000 & 1.000 & 0.950 \\
\hline 25 & $\mathrm{AR}(3)$ & Full & 0.254 & 0.026 & 0.623 & 0.266 \\
\hline 50 & $\mathrm{AR}(3)$ & Full & 0.160 & 0.011 & 0.824 & 0.388 \\
\hline 100 & $\mathrm{AR}(3)$ & Full & 0.112 & 0.002 & 0.983 & 0.829 \\
\hline
\end{tabular}

Table 3.6: Rejection frequencies of the F-test (3.13) at a 5 significance level.

against a decrease in the variance of the estimates decreases as expected on the basis of asymptotic theory.

Next, we analyzed the empirical distribution of the differences between the median RMSFE using unrestricted parameter estimates and the median RMSFE using pooled parameter estimates. In Figures 3.3 and 3.4 we report these distributions for the models with respectively parameter homogeneity and parameter heterogeneity. Information on distributions of the mean and median RMSFE is given in Table 3.7.

As expected as $T$ increases, the distributions become more concentrated. Left-skewness of a distribution means that the median (across countries) RMSFE of the pooled forecasts is larger than that of the forecasts based on unpooled estimates. In column 8 of Table 3.7, we report the percentage number of times forecasts using unpooled estimates outperformed those based on pooled estimates. It is not surprising to see that under parameter homogeneity, pooled forecasts are generally more accurate than unpooled. In particular, when $T=25$, the simulations indicate that pooling is appropriate even under parameter heterogeneity. Even for $T=50$ and under parameter heterogeneity, it seems to be advisable to use pooled forecasts, based on a VAR-model, which leave a major part of the contemporaneous correlation in the disturbances and therefore yields forecasts that are genuinely less accurate than those for the AR(3)LI model. These findings are in line with the theoretical results presented in section 2 and the conclusions drawn above for the $F$-tests

\subsubsection{Analyses of international data}

In this section we present the results of an empirical analysis of the pooling restrictions for model (3.14) and the associated VAR(3) model using international data for eighteen countries.

Tests of the pooling restrictions $H_{0}: R \beta_{a}=0$ against the alternative $H_{1}: R \beta_{a} \neq 0$ are reported in Tables 3.8 and 3.9 for the AR(3)LI model and the AR(3) model respectively 


\begin{tabular}{|c|c|c|c|c|c|c|c|}
\hline \multicolumn{3}{|c|}{ Homogenous } & \multicolumn{2}{|l|}{ Mean } & \multicolumn{3}{|l|}{ Median } \\
\hline$T$ & estimator & $\Sigma$ & Unpooled & Pooled & Unpooled & Pooled & $\% \Delta<0$ \\
\hline 25 & $\mathrm{AR}(3) \mathrm{LI}$ & $\sigma^{2} I$ & 0.0159 & 0.0146 & 0.0157 & 0.0144 & 0.1490 \\
\hline 50 & $\mathrm{AR}(3) \mathrm{LI}$ & $\sigma^{2} I$ & 0.0105 & 0.0101 & 0.0104 & 0.0100 & 0.2720 \\
\hline 100 & AR(3)LI & $\sigma^{2} I$ & 0.0073 & 0.0071 & 0.0073 & 0.0071 & 0.3090 \\
\hline 25 & $\operatorname{AR}(3)$ & $\sigma^{2} I$ & 0.0218 & 0.0207 & 0.0217 & 0.0204 & 0.2120 \\
\hline 50 & $\mathrm{AR}(3)$ & $\sigma^{2} I$ & 0.0149 & 0.0145 & 0.0148 & 0.0144 & 0.2860 \\
\hline 100 & $\operatorname{AR}(3)$ & $a^{2} I$ & 0.0103 & 0.0101 & 0.0102 & 0.0101 & 0.3480 \\
\hline 25 & AR(3)LI & Block & 0.0154 & 0.0147 & 0.0152 & 0.0146 & 0.3090 \\
\hline 50 & $\mathrm{AR}(3) \mathrm{LI}$ & Block & 0.0103 & 0.0102 & 0.0102 & 0.0100 & 0.4110 \\
\hline 100 & $\mathrm{AR}(3) \mathrm{LI}$ & Block & 0.0072 & 0.0071 & 0.0072 & 0.0072 & 0.4200 \\
\hline 25 & $\operatorname{AR}(3)$ & Full & 0.0219 & 0.0208 & 0.0218 & $0.02 \overline{0}$ & 0.2260 \\
\hline 50 & $\operatorname{AR}(3)$ & Full & 0.0149 & 0.0145 & 0.0148 & 0.0144 & 0.2990 \\
\hline 100 & $\mathrm{AR}$ & Full & 93 & 0.0101 & 0.0102 & 0.0101 & 0.3540 \\
\hline \multicolumn{3}{|c|}{ Heterogenous } & \multicolumn{2}{|l|}{ Mean } & \multicolumn{3}{|l|}{ Median } \\
\hline$T$ & estimator & $\Sigma$ & Unpoole & Pooled & Unpooled & Pooled & $\% \Delta<0$ \\
\hline 25 & $\mathrm{AR}(3) \mathrm{LI}$ & $\sigma^{2} I$ & 0.0163 & 0.0160 & 0.0162 & 0.0159 & 0.4480 \\
\hline 50 & AR(3)LI & $\sigma^{2} I$ & 0.0107 & 0.0112 & 0.0107 & 0.0112 & 0.6670 \\
\hline 100 & $\mathrm{AR}(3) \mathrm{LI}$ & $\sigma^{2} I$ & 0.0073 & 0.0078 & 0.0072 & 0.0077 & 0.7820 \\
\hline 25 & $\operatorname{AR}(3)$ & $\sigma^{2} I$ & 0.0174 & 0.0167 & 0.0173 & 0.0166 & 0.3360 \\
\hline 50 & $\operatorname{AR}(3)$ & $\sigma^{2} I$ & 0.0118 & 0.0117 & 0.0118 & 0.0177 & 0.4670 \\
\hline 100 & $A R(3)$ & $\sigma^{2} I$ & 0.0081 & 0.0082 & 0.0080 & 0.0081 & 0.5470 \\
\hline 25 & $\mathrm{AR}(3) \mathrm{LI}$ & Block & 0.0157 & 0.0163 & 0.0156 & 0.0161 & 0.6110 \\
\hline 50 & $\mathrm{AR}(3) \mathrm{LI}$ & Block & 0.0105 & 0.0114 & 0.0105 & 0.0113 & 0.7810 \\
\hline 100 & $\mathrm{AR}(3) \mathrm{LI}$ & Block & 0.0072 & 0.0079 & 0.0071 & 0.0078 & 0.8320 \\
\hline 25 & $\operatorname{AR}(3)$ & Full & 0.0176 & 0.0168 & 0.0173 & 0.0166 & 0.3370 \\
\hline 50 & $\operatorname{AR}(3)$ & Full & 0.0118 & 0.0117 & 0.0118 & 0.0117 & 0.4850 \\
\hline 100 & $\mathrm{AR}(3)$ & Full & 0.0081 & 0.0081 & 0.0081 & 0.0081 & 0.5600 \\
\hline
\end{tabular}

$\% \Delta<0$ equals the percentage of times that the difference between the individual and pooled median RMSFE is smaller than zero

Table 3.7: Mean and Median (Median)RMSFE for the simulations. 

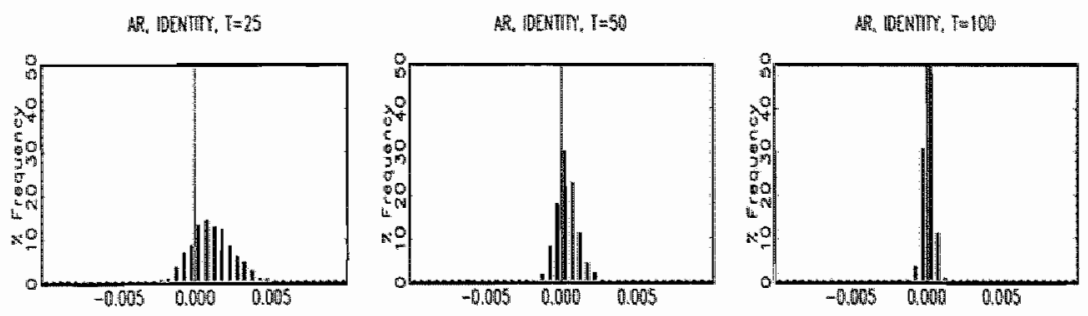

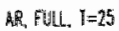

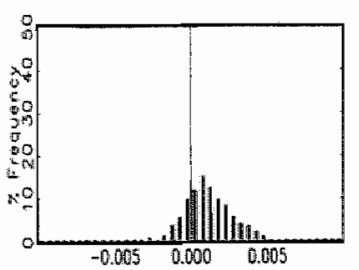

HA. FULL T T 50

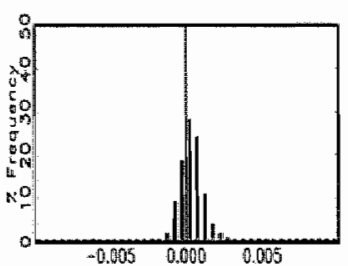

HR, FU, I I ITHOD

ARIU, IDENTIY, I $=25$

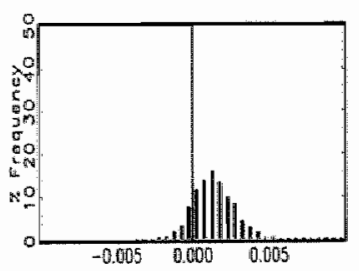

ARL, COENTIT,
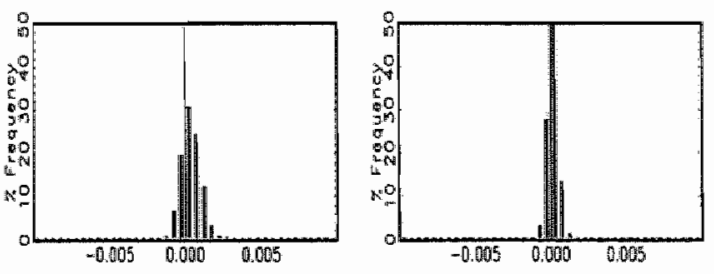

Hatl OWEGA, $1=25$

ALI: OUECh $1 \mathrm{WO} 50$

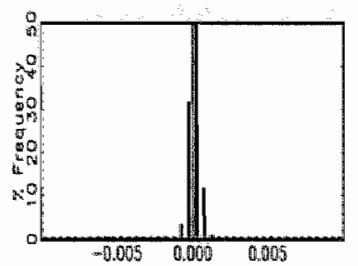

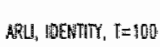
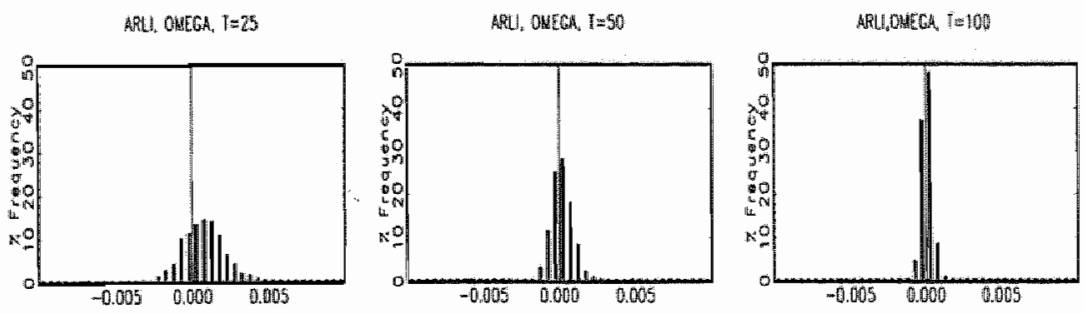

Figure 3.3: Distribution of the simulated differences between the medians of the RMSFEs (unpooled minus pooled) under parameter homogeneity. 

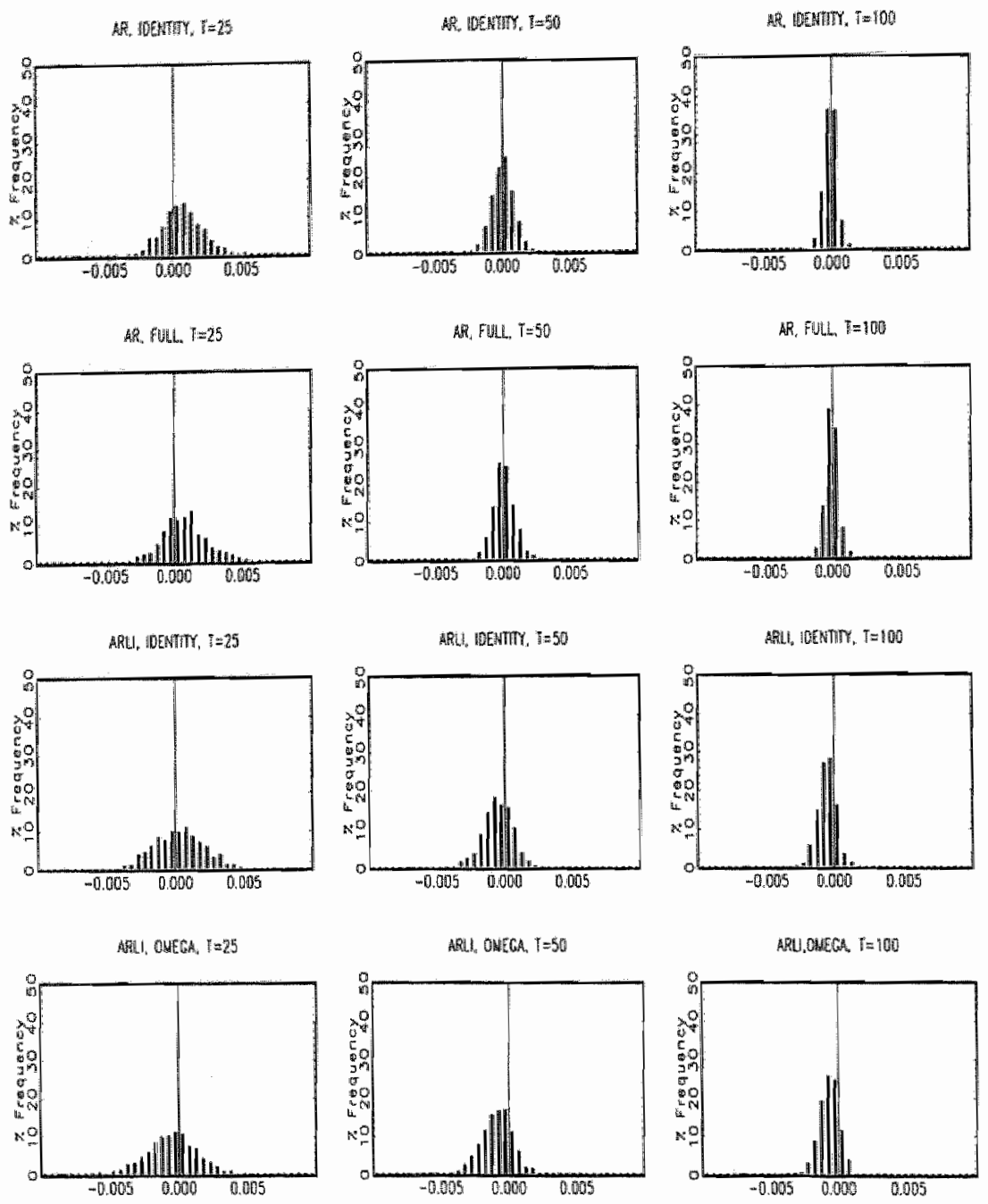

Figure 3.4: Distribution of the simulated differences between the medians of the RMSFEs (unpooled minus pooled) under parameter heterogeneity. 
for estimation periods varying from 1961-1980 to 1961-1990. We report the values of the test statistic $F^{*}$, defined in (3.13), and the $p$-values for the asymptotically justified tests of exact linear restrictions $R \beta_{a}=0, \lambda=0$ and the test whether the second weak mean squared error criterion, $\lambda_{n} \leq q / 2$ holds. The matrix $\Sigma$ is assumed to be respectively $\Sigma=\sigma^{2} I_{N}$, diagonal and block-diagonal (as explained above).

The values of the $F$-statistic are given in column 2 of Tables 3.8 and 3.9. Column 3 contains the value of the noncentrality parameter $\theta_{n}$ given in (3.13). In the columns 4 and 5 , the $p$-values are reported for the tests of $H_{0}: \lambda=0$ vs. $H_{1}: \lambda \neq 0, H_{0}: \lambda \leq q / 2$ against $H_{1}: \lambda>q / 2$. The two criteria correspond to the tests of $R \beta_{a}=0$ and McElroy's and second weak MSE criterion respectively. For the AR(3)LI and the AR(3) models, under the assumption that $\Sigma=\sigma^{2} I_{N}$ or that $\Sigma$ is diagonal, an $F$-test usually does not lead to rejection of the pooling restrictions $R \beta_{a}=0$. Consequently, the less stringent restrictions of the second weak MSE test for pooling given by McElroy (1977) are not rejected either in these two cases. A similar conclusion is reached if the first weak criterion is used to test for pooling. The $p$-values are never lower than .64 when the estimation period is varied from 1961-1980 to 1961-1990. McElroy's second weak criterion for pooling is not rejected in general, except for the $\mathrm{AR}(3)$ model using an unrestricted covariance matrix $\Sigma$. We note that similar conclusions hold for the first weak criterion. Notice also that the pooled $\mathrm{AR}(3)$ model has to be rejected when compared with the pooled AR(3)LI model.

We have to point out that in the likely presence of contemporaneous residual correlation and of heteroscedasticity, the true size of a test based on an " $F$-statistic" with spherical $\Sigma$ will be different from the assumed size when the true $\Sigma$ is nonspherical.

The evidence for the $F$-test in Tables 3.8 and 3.9 for $\Sigma=\sigma^{2} I_{N}$ or $\Sigma$ diagonal supports the null hypothesis as neglecting residual correlation in estimation generally leads to rejection frequencies for the null hypothesis which are much lower than the nominal size of the test (see e.g.. Palm and Sneek (1984) for results on the $F$-test in a regression model when neglecting serial correlation in the disturbances).

When $\Sigma$ is estimated as a block-diagonal matrix $H_{0}: R \beta_{a}=0$ has to be usually rejected at conventional significance levels. The less restrictive second weak MSE error tests do not lead to rejecting $H_{0}$ in this case. For the AR(3) model, with full disturbance covariance matrix the $p$-values for the pooling restrictions are very small. In this case, the second weak MSE criterion also leads to reject pooling (see Table 3.9). It should be noted that we are using asymptotically justified procedures in relatively small samples. The procedures are asymptotically justified because $\Sigma$ has to be estimated and because of the presence of lagged dependent variables among regressors. There is an earlier literature on the incorrect sizes of asymptotic tests indicating that these tests reject the null hypothesis too often in finite samples. The simulations in section 3.3.2 however indicate that asymptotic theory provides rather good guidance in small samples. F-statistics neglecting the presence of residual correlation are expected to reject the null hypothesis less often than they should according to the nominal size. Therefore, we conclude that on the whole the evidence from Tables 3.8 and 3.9 supports the pooling restrictions.

This conclusion is supported by the results given in Table 3.10. For the forecast period 1983-90, pooling leads to a substantial reduction in the RMSFE for the AR(3)LI model when a pooled GLS estimator is used with respectively estimated full and block-diagonal covariance matrix. 


\begin{tabular}{|c|c|c|c|c|}
\hline \multicolumn{5}{|l|}{$\Sigma: \sigma^{2} I$} \\
\hline year & $\bar{F}$-value & $\theta$ & p-value for $\lambda=0$ & p-value for $\lambda=68$ \\
\hline 1981 & 1.1096 & $4 . \overline{1376}$ & 0.2427 & $0 . \overline{9777}$ \\
\hline 1982 & 1.0574 & 3.8120 & 0.3497 & 0.9907 \\
\hline 1983 & 1.0974 & 7.8941 & 0.2600 & 0.9833 \\
\hline 1984 & 0.9712 & 6.9658 & 0.5715 & 1.0000 \\
\hline 1985 & 0.9707 & 7.0353 & 0.5733 & 1.0000 \\
\hline 1986 & 0.9702 & 6.7933 & 0.5750 & 1.0000 \\
\hline 1987 & 0.9382 & 6.5314 & 0.6633 & 1.0000 \\
\hline 1988. & 1.0111 & 7.0277 & 0.4605 & 0.9972 \\
\hline 1989 & 1.0259 & 8.3497 & 0.4194 & 0.9964 \\
\hline 1990 & 1.0422 & 8.1066 & 0.3754 & 0.9953 \\
\hline \multicolumn{5}{|c|}{$\Sigma$ : diagonal } \\
\hline 1981 & 1.3858 & 10.3503 & 0.0148 & 0.7009 \\
\hline 1982 & 1.2044 & 9.8716 & 0.1038 & 0.9311 \\
\hline 1983 & 1.2778 & 9.6392 & 0.0461 & 0.8635 \\
\hline 1984 & 1.2006 & 12.4582 & 0.1018 & 0.9377 \\
\hline 1985 & 1.1535 & 11.8474 & 0.1572 & 0.9660 \\
\hline 1986 & 1.1353 & 10.1420 & 0.1830 & 0.9744 \\
\hline 1987 & 1.0575 & 9.4574 & 0.3402 & 0.9929 \\
\hline 1988 & 1.1041 & 8.5913 & 0.2353 & 0.9850 \\
\hline 1989 & 1.1063 & 10.0359 & 0.2293 & 0.9849 \\
\hline 1990 & 1.1488 & 10.1247 & 0.1540 & 0.9722 \\
\hline \multicolumn{5}{|c|}{$\sum:$ Block } \\
\hline 1981 & 1.6257 & 4.8867 & 0.0006 & 0.2886 \\
\hline 1982 & 1.3376 & 4.7501 & 0.0244 & 0.7805 \\
\hline 1983 & 1.6307 & 5.3224 & 0.0004 & 0.2750 \\
\hline 1984 & 1.5227 & 2.2402 & 0.0017 & 0.4525 \\
\hline 1985 & 1.3511 & 5.2240 & 0.0171 & 0.7661 \\
\hline 1986 & 1.4116 & 5.8129 & 0.0070 & 0.6627 \\
\hline 1987 & 1.1629 & 5.5796 & 0.1393 & 0.9637 \\
\hline 1988 & 1.0406 & 6.0494 & 0.3814 & 0.9950 \\
\hline 1989 & 0.9469 & 7.4391 & 0.6413 & 1.0000 \\
\hline 1990 & 0.9508 & 7.3449 & 0.6307 & 1.0000 \\
\hline
\end{tabular}

Table 3.8: Testing for pooling in the AR(3)LI model. 


\begin{tabular}{|c|c|c|c|c|}
\hline \multicolumn{5}{|l|}{$\Sigma: \sigma^{2} I$} \\
\hline year & F-value & $\bar{\theta}$ & $p$-value for $\lambda=0$ & $p$-value for $\lambda=34$ \\
\hline 1981 & 0.9160 & 5.9719 & 0.6617 & 0.9944 \\
\hline 1982 & 0.7816 & 5.5208 & 0.8903 & 1.0000 \\
\hline 1983 & 0.7503 & 7.5922 & 0.9246 & 1.0000 \\
\hline 1984 & 0.7770 & 7.7367 & 0.8973 & 1.0000 \\
\hline 1985 & 0.7822 & 7.5106 & 0.8918 & 1.0000 \\
\hline 1986 & 0.7920 & 7.3827 & 0.8802 & 1.0000 \\
\hline 1987 & 0.8240 & 7.2331 & 0.8355 & 1.0000 \\
\hline 1988 & 0.8066 & 7.5548 & 0.8618 & 1.0000 \\
\hline 1989 & 0.8411 & 7.4793 & 0.8089 & 1.0000 \\
\hline 1990 & 0.8600 & 7.4207 & 0.7762 & 1.0000 \\
\hline \multicolumn{5}{|c|}{$\Sigma$ : diagonal } \\
\hline 1981 & 1.2102 & 10.2665 & 0.1434 & 0.8691 \\
\hline 1982 & 0.9846 & 10.6773 & 0.5163 & 0.9856 \\
\hline 1983 & 0.8835 & 10.0391 & 0.7284 & 0.9969 \\
\hline 1984 & 0.9364 & 9.6711 & 0.6200 & 0.9929 \\
\hline 1985 & 0.9418 & 9.3295 & 0.6086 & 0.9925 \\
\hline 1986 & 0.8687 & 9.6048 & 0.7583 & 0.9977 \\
\hline 1987 & 0.8731 & 9.0973 & 0.7504 & 0.9976 \\
\hline 1988 & 0.8193 & 9.7261 & 0.8433 & 1.0000 \\
\hline 1989 & 0.8573 & 9.4925 & 0.7808 & 1.0000 \\
\hline 1990 & 0.8610 & 9.4855 & 0.7745 & 1.0000 \\
\hline \multicolumn{5}{|l|}{$\Sigma$ : Full } \\
\hline 1981 & 4.5471 & 4.3782 & 0.0000 & 0.0000 \\
\hline 1982 & 3.7992 & 5.0988 & 0.0000 & 0.0000 \\
\hline 1983 & 2.4048 & 4.9766 & 0.00000 & 0.0024 \\
\hline 1984 & 1.7547 & 4.7972 & 0.0006 & 0.1757 \\
\hline 1985 & 1.6148 & 4.6653 & 0.0029 & 0.3243 \\
\hline 1986 & 2.4875 & 5.3748 & 0.0000 & 0.0010 \\
\hline 1987 & 2.1577 & 5.5885 & 0.0000 & 0.0140 \\
\hline 1988 & 2.0069 & 5.7174 & 0.0000 & 0.0393 \\
\hline 1989 & 2.1248 & 5.7170 & 0.0000 & 0.0170 \\
\hline 1990 & 2.1710 & 5.5343 & 0.0000 & 0.0119 \\
\hline
\end{tabular}

Table 3.9: Testing for pooling in the AR(3) model. 
For the forecast period $1983-90$, GLS-based unpooled forecasts perform slightly worse than OLS-based unpooled forecasts.

\subsection{Conclusions}

In this chapter we studied the problem whether forecasts of a set of panel data generated by models with similar but not necessarily identical parameter structures can be improved by using pooled parameter estimates. Results obtained by McElroy (1977) for a regression model with non-spherical disturbances can be generalized in a straightforward way to apply to systems of regression models used to study panel data with large $T$ and fixed $N$.

The gain in forecast and estimator performance measured by the reduction in MSFE or MSE results from a trade-off between the bias implied by the use of (slightly) false pooling restrictions and the reduction in the covariance matrix of the estimators due to imposing these restrictions. Also as the sample size increases, the covariance matrices of restricted and unrestricted estimates converge to constant matrices but the bias of the restricted estimator (multiplied by $\sqrt{n}$ ) increases without bound. Therefore, beyond some given sample size, the forecasts based on unrestricted estimates will outperform the pooled forecasts.

Our simulation results show that for small and moderate values of $T$, reductions in MSFE can be achieved through pooling, even under parameter heterogeneity. The asymptotic properties of the pooling criteria put forward by McElroy (1977) provide a fairly accurate insight into their properties for finite $T$.

We applied these results to growth rates for 18 OECD countries for the periods starting in the 1950's until 1991 using models put forward by Garcia-Ferrer et al. (1987) and Zellner and Hong (1989). Our empirical results can be summarized as follows.

First, there is contemporaneous residual correlation in the form of a block diagonal structure corresponding to regional groups present in the models for the 18 countries.

Second, when formally tested using an estimated residual covariance matrix, the pooling restrictions and the MSE-criteria for pooling put forward by McElroy (1977) are rejected only for the AR(3) model. They are not rejected when a diagonal or scalar residual covariance matrix is used. We should bear in mind that asymptotically justified $F$-tests tend to reject too often in finite samples. Also, the pooling restrictions and MSE-criteria for 18 countries were jointly tested even not allowing for individual fixed effects in the form of country-specific intercepts. Comparing pooled and unpooled models using posterior odds is probably a sensible alternative in relatively small samples to asymptotically justified test criteria.

Third, in actual forecasting, the median MSFE of OLS-based pooled forecasts is found to be smaller than that of OLS-based individual forecasts. A fairly large sample size is needed for the OLS-based pooled forecasts to be outperformed by a forecast based on unrestricted estimates. Using unpooled GLS with an estimated residual covariance matrix leads to slightly improved forecast performance. Pooled GLS-based forecasts have a much lower median MSFE than pooled OLS-based forecasts. Results for shrinkage forecasts lie between the two polar cases of forecasts based on unrestricted estimates and those based on pooled estimates. This finding parallels results obtained by Blattberg 


\begin{tabular}{|c|c|c|c|c|c|c|}
\hline $\mathrm{AR}(3) \mathrm{LI}$ & \multicolumn{2}{|c|}{ Block } & \multicolumn{2}{|c|}{ Diagonal } & \multicolumn{2}{|c|}{$\sigma^{2} I$} \\
\hline country & unpooled & poolled & unpooled & pooled & unpooled & pooled \\
\hline $\operatorname{can}$ & 3.5823 & 2.5757 & 3.5726 & 2.2619 & 3.5726 & 2.2582 \\
\hline usa & 2.4540 & 2.7546 & 2.6431 & 2.3452 & 2.6431 & 2.3891 \\
\hline aal & 4.2322 & 3.0851 & 3.9689 & 2.7281 & 3.9689 & 2.6749 \\
\hline jap & 2.9103 & 1.5189 & 3.2061 & 0.8999 & 3.2061 & 0.8674 \\
\hline den & 3.6520 & 3.2428 & 3.4620 & 2.9051 & 3.4620 & 2.9456 \\
\hline fin & 5.1698 & 3.0735 & 4.2740 & 2.4980 & 4.2740 & 2.5036 \\
\hline nor & 4.3610 & 3.4589 & 4.0129 & 3.5132 & 4.0129 & 3.541 .4 \\
\hline swe & 2.1673 & 2.6456 & 2.0758 & 2.2765 & 2.0758 & 2.2725 \\
\hline bel & 2.9388 & 2.6704 & 3.1911 & 2.0312 & 3.1911 & 2.0544 \\
\hline fra & 3.2852 & 2.7677 & 1.7459 & 2.2189 & 1.7459 & 2.2772 \\
\hline ger & 3.4362 & 2.6773 & 3.2099 & 2.6125 & 3.2099 & 2.6942 \\
\hline net & 2.2362 & 2.2716 & 2.0300 & 1.8057 & 2.0300 & 1.8840 \\
\hline ire & 4.0171 & 2.9419 & 4.0991 & 2.3556 & 4.0991 & 2.3757 \\
\hline $\mathrm{ukm}$ & 6.4017 & 4.0989 & 5.6718 & 3.9304 & 5.6718 & 3.8935 \\
\hline aus & 2.1660 & 2.1725 & 2.5230 & 1.7571 & 2.5230 & 1.7371 \\
\hline swi & 2.1496 & 2.5038 & 2.2335 & 1.9483 & 2.2335 & 1.9481 \\
\hline ita & 3.9755 & 2.2401 & 3.4932 & 1.9471 & 3.4932 & 1.9939 \\
\hline spa & 3.6313 & 1.7012 & 3.4253 & 1.2590 & 3.4253 & 1.2305 \\
\hline median & 3.5093 & 2.6738 & 3.3176 & 2.2692 & 3.3176 & 2.2748 \\
\hline $\operatorname{AR}(3)$ & \multicolumn{2}{|c|}{ Full } & \multicolumn{2}{|c|}{ Diagonal } & \multicolumn{2}{|l|}{$\sigma^{2} I$} \\
\hline country & unpooled & pooled & unpooled & pooled & unpooled & pooled \\
\hline can & 2.6367 & 1.9434 & 2.8106 & 2.1640 & 2.8106 & 2.1683 \\
\hline usa & 2.0436 & 1.8023 & 2.1283 & 2.2258 & 2.1283 & 2.2728 \\
\hline $\mathrm{aall}$ & 3.3234 & 3.6949 & 3.6873 & 3.5297 & 3.6873 & 3.4976 \\
\hline jap & 1.9710 & 0.7899 & 0.7781 & 0.9139 & 0.7781 & 0.8821 \\
\hline den & 11.8887 & 1.6896 & 2.1340 & 1.6459 & 2.1340 & 1.7240 \\
\hline fin & 2.2841 & 2.3101 & 2.1880 & 2.2620 & 2.1880 & 2.2595 \\
\hline nor & 7.1402 & 2.1947 & 4.3620 & 2.3095 & 4.3620 & 2.3376 \\
\hline swe & 2.8313 & 2.0196 & 1.8720 & 1.8784 & 11.8720 & 1.9054 \\
\hline bel & 2.4269 & 2.0052 & 1.3865 & 1.5290 & 1.3865 & 1.514 .5 \\
\hline fra & 3.1251 & 1.6504 & 1.2129 & 1.2676 & 1.2129 & 1.2663 \\
\hline ger & 3.6212 & 2.9584 & 2.6447 & 2.7138 & 2.6447 & 2.7021 \\
\hline net & 4.2353 & 1.3171 & 1.2344 & 0.8798 & 1.2344 & 0.8708 \\
\hline ire & 5.0117 & 1.9061 & 2.9443 & 1.6905 & 2.9443 & 1.6638 \\
\hline ukm & 2.8309 & 2.5645 & 2.4526 & 2.5469 & 2.4526 & 2.5689 \\
\hline aus & 1.8937 & 1.1040 & 0.9527 & 0.7966 & 0.9527 & 0.7851 \\
\hline swi & 1.6948 & 1.4109 & 1.4720 & 1.0925 & 1.4720 & 1.1009 \\
\hline ita. & 1.2913 & 1.3645 & 1.4611 & 1.1073 & 1.4611 & 1.1080 \\
\hline spa & 3.0048 & 1.1796 & 0.9246 & 1.0402 & 0.9246 & 1.0201 \\
\hline median & 2.7338 & 1.8542 & 2.0001 & 1.6682 & 2.0001 & 1.6939 \\
\hline
\end{tabular}

Table 3.10: Root mean square forecast errors for the $A R(3) L I$ and $A R(3)$ models. 
and George (1991). When modeling sales using a chain-brand model, they found that GLS added little with their data whereas pooling and shrinkage estimation procedures provided superior estimates to OLS. Our analysis shows that for the samples availlable the parameter structures in the models for the real GDP growth rates in the 18 countries are sufficiently similar so that the pooled forecasts dominate unpooled forecasts when OLS or GLS is used, a finding that is in line with previous results by Garcia et al. (1987), Zellner and Hong (1989) and Min and Zellner (1993) among others. Finally, the question whether restricting the contemporaneous residual correlations to be the same within groups of countries and possibly across groups leads to further improvement of GLS-based pooled forecasts remains to be investigated.

\subsection{Data appendix}

The used data set is an updated set as used by Min and Zellner (1993) and consists of annual postwar data for eighteen OECD countries for the period 1948 to 1990 . The data are obtained from the main International Monetary Funds International Financial Statistics Data Base and contains the following four variables: (i) real stock prices, (ii) a consumer price index, (iii) nominal money M1 and (iv) GDP. Because of missing values and to make a fair comparison between the $\mathrm{AR}(3)$ model and the AR(3)LI model, we only included those years which could be used to estimate both models. The following table gives the starting year 


\begin{tabular}{|l|c|}
\hline Country & Starting year \\
\hline Australia & 1956 \\
\hline Austria & 1949 \\
\hline Belgium & 1953 \\
\hline Canada & 1955 \\
\hline Denmark & 1950 \\
\hline Finland & 1950 \\
\hline France & 1950 \\
\hline Germany & 1950 \\
\hline Ireland & 1948 \\
\hline Italy & 1951 \\
\hline Japan & 1953 \\
\hline The Netherlands & 1950 \\
\hline Norway & 1949 \\
\hline Spain & 1954 \\
\hline Sweden & 1950 \\
\hline Switzerland & 1953 \\
\hline United Kingdom & 1957 \\
\hline USA & 1955 \\
\hline
\end{tabular}

Table 3.11: Observations available per country. 


\section{Chapter 4}

\section{Multiple Vector Autoregressions and Impulse Response Analysis}

\subsection{Introduction}

In macro-economics there are many research questions which ask for a multi country analysis using dynamic models, especially in areas as international trade, growth and (regional) convergence. The data avajlable in these studies have dimensions which restrict the researchers who are willing to conduct a time series analysis. The number of observations for each individual is often too limited to give statistical tests enough statistical power or even to identify some parameters of interest.

Therefore one often puts in additional information obtained from the cross-sectional dimension, i.e. from the observations on the same variables from other individuals. The choice is then restricted between conducting a form of pooled time series analysis, if the number of individuals, $N$, is fixed and small, and panel data analysis if the number of individuals is large and the number of observed time periods, $T$, is small.

In this chapter we consider problems for which $T$ is large enough to assume that $T$ tends to infinity and the number of individuals is small compared to $T$, i.e. we assume $T$ to be larger then $N$ and the latter to be fixed. A typical data set is characterized by having dimensions such that the number of individuals is a fraction of the number of observed time periods, e.g., 3 or more variables for 5 individuals, observed for 30 time periods.

We introduce stationary vector autoregressive models in which each individual has an individual nonrandom time invariant part (intercept, fixed effect) and a covariance matrix of the innovations, which can be decomposed into individual specific parameters and parameters common to all individuals. The lag coefficients are common to all individuals. The fact that we assume $N$ to be small and fixed and $T$ to be large implies that the analysis can be interpreted as time series analysis with restrictions between the models for the individuals. Observe that if $T$ is really large then there is no need for imposing the restrictions. In that case we are able to estimate sufficiently accurately the parameters of interest using only the observations of the individual of interest. The assumption of large $T$ is imposed as it is assumed that the asymptotics based on large $T$ are more appropriate than those based on large $N$. 
The motivation for such a model lies in the fact that there are many instances in which one cannot analyze a complete VAR model due to the lack of observations. Using a model as described above and in more detail below we will be able to do a full scale analysis of the common dynamics. As an application we develope impulse response analysis based on this type of models.

Another motivation to impose restrictions across individuals can be the fact that sometimes it is reasonable to assume parameters to be constant and reasonably similar across entities during a shorter period of time as opposed to parameter constancy and similarity for a long period of time. When estimating two similar models for instance for the USA and Canada from before and after the 1973 Oil crisis, is it more appropriate to analyze the countries separately using the data for the complete observation period or to pool the data for the two countries but to distinguish two different subperiods separated by the Oil crisis in 1973? The answer is not directly clear.

The choice of relying on large $T$ asymptotics with $N$ being fixed enables us to use all the usual tools of time series analysis. Keeping $T$ fixed small and $N$ large would make it necessary to make use of the tools developed for panel data analysis. We refer to the article by Holz-Eakin et al. (1988) for this latter analysis. Observe that for dynamical models there are no results yet for the finite sample case, the frequently most appropriate one, that both $N$ and $T$ are fixed.

As an application we analyze the reduced form of the money demand for six European countries. Using the models and tests proposed in this chapter we find that we can indeed impose cross-country restrictions on the lag matrices and contemporaneous covariance matrix. Imposing these restrictions leads as expected to a gain in precision in the analysis of the impulse response functions, the confidence bounds get much tighter.

In terms of scope, we limit ourselves in this chapter to weakly stationary processes. Nonstationary processes, possibly cointegrated are not considered and left for further research. The models, restrictions and subsequent analysis can however be extended to the case of nonstationary processes. To include this extension in this chapter would lengthen this already lengthy exposition even more. Nonstationary processes have for instance been studied by Lütkepohl and Reimers (1992).

The chapter is organized as follows. In section two we formally introduce the models and discuss motivation and implications. In section three we consider some general theory concerning a single vector autoregression with the aim to compare the results with the results for the multiple vector autoregressions. In section four we derive Maximum Likelihood Estimators and Least Squares Estimators as well as the asymptotic properties of these estimators. In section five we consider impulse response analysis while in section six testing within this class of models is studied. In section seven we present the analysis of a multiple VAR system of three key macroeconomic variables for Austria, Belgium, Germany, The Netherlands, The United Kingdom and Switzerland simultaneously.

\subsection{The models}

The model for each individual is a VAR of order $p$. By allowing for different intercepts across individuals and restricting the lag matrices across individuals the model enables us 
to account for specific individual effects like an individual mean and/or individual scale effects and common dynamics across individuals. The model, apart from the covariance structure of the innovations reads as follows;

$$
y_{i, t}=c_{i}+\Phi_{1} y_{i, t-1}+\ldots+\Phi_{p} y_{i, t-p}+\varepsilon_{i, t}, \quad i=\{1,2, \ldots, N\}
$$

where $y_{i, t}$ is a $(k \times 1)$ vector of observed variables, $y_{i, t}=\left(y_{i, t}^{1}, \ldots, y_{i, t}^{k}\right)^{\prime}, c_{i}=\left(c_{i}^{1}, \ldots, c_{i}^{k}\right)^{\prime}$ a

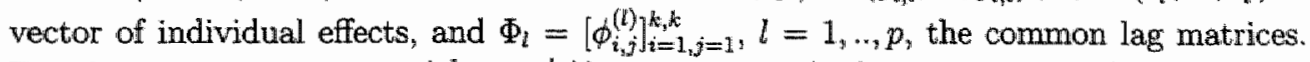
For the innovations $\varepsilon_{i, t}=\left(\varepsilon_{i, t}^{1}, \ldots, \varepsilon_{i, t}^{k}\right)^{\prime}$ we assume $E\left(\varepsilon_{i, t}\right)=0$, thereby identifying the intercept.

To identify the parameters in the model we have to specify further assumptions on the innovations. Assuming normality of the innovations the specification of the covariance structure is sufficient for complete identification. We assume the innovations to be uncorrelated across individuals and across time, i.e.,

$$
E\left(\varepsilon_{i, k} \varepsilon_{i, s}^{\prime}\right)=0 \quad s \neq t, \quad E\left(\varepsilon_{i, t} \varepsilon_{j, s}^{\prime}\right)=0 \quad i \neq j, \quad s, t \in\{1, \ldots, T\}
$$

For the symmetric positive definite $k \times k$ covariance matrix of the innovations for individual $i$ at time $t, E\left(\varepsilon_{i, t} \varepsilon_{i, t}^{\prime}\right)=\Omega_{i}$, we consider four possible parametrizations;

1. The covariance matrix is equal across individuals,

$$
\Omega_{i}=\Omega,
$$

with $\Omega$ symmetric positive definite. This is the most restrictive assumption. We denote this covariance structure with $\Omega^{p}$.

2. The covariance matrix is allowed to differ across individuals. The only requirement for the individual covariance matrix $\Omega_{i}$, is being symmetric and positive definite. This is the least restrictive of the parametrizations. Denote this covariance structure with $\Omega^{i}$.

3. The symmetric positive definite covariance matrix can be decomposed into common and individual effects based on the Choleski decomposition of a real positive definite symmetric matrix

$$
\Omega_{i}=\Gamma \Sigma_{i}^{2} \Gamma^{\prime}
$$

where the matrix $\Gamma$ is lower triangular with ones on the diagonal and $\Sigma_{i}$ is diagonal. Observe that this parametrization implies that the diagonal of $\Sigma_{i}$ contains the standard deviations of the disturbances of individual $i$. The matrix $\Gamma$ is restricted to be the same for all $i$. This covariance structure is denoted by $\Omega^{s b}$. The superscript $s b$ is an abbreviation for scaled between. This notation is explained at the end of this section.

4. A second decomposition of the symmetric positive definite covariance matrix into common and individual parameters is given by

$$
\Omega_{i}=\Sigma_{i} \Gamma \Gamma^{\prime} \Sigma_{i}
$$


again based on the Choleski decomposition ${ }^{1}, \Gamma$ being lower triangular with ones on the main diagonal and the $\Sigma_{i}^{\prime} s$ being diagonal. Again the $\Sigma_{i}^{\prime} s$ contain the standard deviations of the disturbances for individual $i$ and the matrix $\Gamma$ is restricted to be the same for all $i$. We denote this covariance structure with $\Omega^{s w}$. The superscript $s w$ is an abbreviation for scaled within. This notation is explained at the end of this section.

Observe that the above parametrizations (1), (3) and (4) only imply restrictions in case we are considering models with more than one individual. In case of one individual the parametrizations are just reparametrizations. Before discussing the details and motivation for these parametrizations we state the assumption that limits the subsequent analysis to that of stationary vector autoregressive processes. We thereby exclude explosive processes and unit roots and the possibility of cointegrating relationships. We assume the model for each individual time series to be stable, i.e.

$$
\operatorname{det}\left(I-\Phi_{1} z-\ldots-\Phi_{p} z^{p}\right) \neq 0, \text { for }|z| \leq 1 .
$$

We note that it is possible to extend the results below to the above mentioned nonstationary cases but we will not discuss these here.

We will denote the model characterized by $\Omega^{p}, \Omega^{i}, \Omega^{s b}$ and $\Omega^{s w}$ by the $\mathrm{p}($ ooled), i(individual),$- \mathrm{s}$ (caled)b(etween)- and the $\mathrm{s}$ (caled)w(ithin)model respectively. In all models each individual has the same lag polynomial. This gives us the opportunity of obtaining a pooled estimate of the lag polynomial with, when the restrictions are approximately valid, more precision. It further enables us to model higher order systems than in an individual time series model. Pooling of all parameters however would lead to a too rigid model. Therefore we introduce a number of individual parameters to enable the model to account for some specific aspects of an individual.

We firstly allow for an individual nonrandom intercept. This gives each individual a possibly different mean. In the panel literature this is known as a fixed effects model, see for instance Baltagi (1995) or Hsiao (1986). We treat the individual effects as fixed as we are usually considering nonrandom groups of individuals.

Note that we do not allow for contemporaneous correlation between individuals but only within the model for individual i, i.e. between the variables of individual $i$. Also we assume the dynamics of the model to be specified correctly i.e. that there is no autocorrelation left between the innovations of one individual through time.

Some further comments on the differences and similarities between the models are in order. The choice of the parametrizations for the covariance matrices has been guided by two leading arguments; reduction of the number of parameters and solving problems of scaling effects. The inclusion of $\Omega^{i}$ in the analysis is for the reason that this model nests all other models and leaves room to develop tests for the other parametrizations. The completely pooled covariance matrix $\Omega^{p}$ is the most sparse parametrization and is not flexible enough to cope with problems of scale, like different units, measures etc. Note that in this model only the intercept is allowed to differ across individuals.

\footnotetext{
${ }^{1}$ Observe that this is always possible: Use the Choleski decomposition $\Omega_{i}=L_{i} L_{i}^{\prime}$ where $L_{i}$ is lower triangular with positive elements on the main diagonal. Then in turn we can always decompose $L_{i}=$ $P_{i} Q=Q^{*} P_{i}$ where $P_{i}$ is diagonal and $Q$ and $Q^{*}$ are lower triangular.
} 
To provide insight on how the parametrizations of $\Omega^{s b}$ and $\Omega^{s w}$ work out we restate both models by using the fact that we can replace the restriction on the covariance matrices with equivalent restrictions on the lag polynomial coefficients. For the sb-model we obtain, suppressing the individual mean,

$$
\Sigma_{i}^{-1} \Gamma^{-1} y_{i, t}=\Sigma_{i}^{-1} \Gamma^{-1} \Phi_{1} y_{i, t-1}+\ldots+\Sigma_{i}^{-1} \Gamma^{-1} \Phi_{p} y_{i, t-p}+u_{i, t},
$$

with $u_{i, t}=\Sigma_{i}^{-1} \Gamma^{-1} \varepsilon_{i, t}$ and $E\left(u_{i, t} u_{i, t}{ }^{\prime}\right)=I_{k}$. For the sw-model obtain

$$
\Gamma^{-1} \Sigma_{i}^{-1} y_{i, t}=\Gamma^{-1} \Sigma_{i}^{-1} \Phi_{1} y_{i, t-1}+\ldots+\Gamma^{-1} \Sigma_{i}^{-1} \Phi_{p} y_{i, t-p}+u_{i, t},
$$

with $u_{i, t}=\Gamma^{-1} \Sigma_{i}^{-1} \varepsilon_{i, t}$ and $E\left(u_{i, t} u_{i, t}{ }^{\prime}\right)=I_{k}$.

The different parametrizations of the covariance matrices induce heterogeneity among the individuals in two completely different ways. To see this we observe that we have in case $k=3$ for the sb-model

$$
\Sigma_{i}^{-1} \Gamma^{-1}=\left[\begin{array}{ccc}
\sigma^{i, 1} & 0 & 0 \\
\sigma^{i, 2} \gamma^{2,1} & \sigma^{i, 2} & 0 \\
\sigma^{i, 3} \gamma^{3,1} & \sigma^{i, 3} \gamma^{3,2} & \sigma^{i, 3}
\end{array}\right]
$$

This implies that for each individual every equation is normalized by the standard deviation of the corresponding innovation after orthogonalization. However, for the sw-model we have

$$
\Gamma^{-1} \Sigma_{i}^{-1}=\left[\begin{array}{ccc}
\sigma^{i, 1} & 0 & 0 \\
\sigma^{i, 1} \gamma^{2,1} & \sigma^{i, 2} & 0 \\
\sigma^{i, 1} \gamma^{3,1} & \sigma^{i, 2} \gamma^{3,2} & \sigma^{i, 3}
\end{array}\right]
$$

This implies that for each individual every equation is normalized by the standard deviation of the corresponding innovation before orthogonalization. The reason why we are denoting this model with $s$ (caled)w(ithin)-model is that this parametrization also implies scaling of the pooled autoregressive parameters within the equations of one individual. As in the sb-model we only normalize between equations we denote it the $s$ (caled)b(between)model.

Another observation is relevant when we interpret the models as a structural VAR. Then the sb-model is similar to a recursive system, due to the triangularity of $\Gamma$, with pooled lag coefficients and a diagonal contemporaneous covariance matrix. To see this we write down the case $p=1$

$$
y_{i, t}=\left(I-\Sigma_{i}^{-1} \Gamma^{-1}\right) y_{i, t}+\Sigma_{i}^{-1} \Gamma^{-1} \Phi_{1} y_{i, t-p}+u_{i, t} .
$$

We note that this can also be used to put further restrictions on the $\Gamma$ implied by economic theory. The sw-model can also be interpreted as a recursive system

$$
y_{i, t}=\left(I-\Gamma^{-1} \Sigma_{i}^{-1}\right) y_{i, t}+\Gamma^{-1} \Sigma_{i}^{-1} \Phi_{1} y_{i, t-p}+u_{i, t},
$$

but now the lag polynomial is not the same for each individual. 


\subsection{Theory for single vector autoregressions}

Before we discuss (conditional) Maximum Likelihood and Least Squares estimation of the above described models we first state some results that will be used in the remaining part of the chapter. We note that the notation used in this section is independent from the other sections of this chapter.

Assume the following model to hold for the $k$-variate process $y_{t}=\left(y_{t}^{(1)}, \ldots, y_{t}^{(k)}\right)^{s}$

$$
y_{t}=c+\Phi_{1} y_{t-1}+\ldots+\Phi_{p} y_{t-p}+\varepsilon_{t}
$$

with for the independent normally distributed innovations $E\left(\varepsilon_{t}\right)=0, E\left(\varepsilon_{t} \varepsilon_{t}^{\prime}\right)=\Sigma$. Further we assume that the autoregression is stable, i.e.

$$
\operatorname{det}\left(I-\Phi_{1} z-\ldots-\Phi_{p} z^{P}\right) \neq 0, \text { for }|z| \leq 1 .
$$

This implies that the process is second order stationary. We can now introduce the mean $\mu=\left(I-\Phi_{1}-\ldots-\Phi_{p}\right)^{-1} c$ and rewrite (4.11) as

$$
y_{t}-\mu=\sum_{j=1}^{p} \Phi_{j}\left(y_{t-j}-\mu\right)+\varepsilon_{t}
$$

From Wolds Theorem we know that every second order stationary process has a moving average representation

$$
y_{t}-\mu=\sum_{j=0}^{\infty} \Theta_{j} \varepsilon_{t-j} .
$$

Here the $k \times k$ matrices $\Theta_{j}$ contain the MA coefficients and $\Theta_{0}=I_{k}$. These coefficients can be derived in the following manner. Introduce the backshift operator $L$ we then see that we have

$$
\left(I_{k}-\sum_{j=1}^{p} \Phi_{j} L^{j}\right)\left(y_{t}-\mu\right)=\varepsilon_{t}
$$

and

$$
y_{t}-\mu=\left(\sum_{j=0}^{\infty} \Theta_{j} L^{j}\right) \varepsilon_{t},
$$

which leads to the crucial relation

$$
\left(I_{k}-\sum_{j=1}^{p} \Phi_{j} L^{j}\right)\left(\sum_{j=0}^{\infty} \Theta_{j} L^{j}\right)=I_{k} .
$$

Using this relation we can calculate $\Theta_{j}$ recursively:

$$
\begin{aligned}
& \Theta_{0}=I_{k}, \\
& \Theta_{j}=\sum_{n=1}^{j} \Theta_{j-n} \Phi_{n} \quad j \geq 1 .
\end{aligned}
$$


Observe that it is also possible to invert $\left(I_{k}-\sum_{j=1}^{p} \Phi_{j} L^{j}\right)$ in (4.15) directly, however this operation is not always easy to perform.

We notice an important fact in connection with the model we introduced in the previous section. As we can see in (4.18) $\mu$ is not directly involved in the construction of the MA lag matrices. This implies that the statistical properties of the MA coefficients are completely determined by the statistical properties of the coefficients of the AR lag. polynomial. In our model we have pooled or restricted estimates for the lag polynomial and so we can hope to obtain more precise impulse responses than based on the observations of one individual. The individual means $\mu_{i}$, which we can estimate with relatively less precision than the lag matrices do not influence the precision of the estimated MA coefficients directly.

Impulse response analysis is the analysis in dynamical systems which explores the effect of an unanticipated one-time shock in the system. In linear second order stationary systems this can be done by means of the MA representation (4.14) of the system. The response of variable $l$ to a unit shock in variable $s$ after $j$ time periods is represented by the parameter $\Theta_{j}^{s, l}$, the $s, l$ the parameter of the $j-t h \operatorname{lag}$ matrix. When the restrictions introduced in the previous section indeed lead to a gain in precision for estimating the parameters of the AR lag matrices the impulse responses also gain precision. In the rest of the chapter we investigate whether this gain really exists and whether the gain can be made useful in practice.

Instead of obtaining the impulse responses from (4.14) it is often also useful to obtain impulse responses for orthogonal impulses. These are obtained from

$$
\begin{aligned}
y_{t}-\mu & =\sum_{j=0}^{\infty} \Theta_{j} W W^{-1} \varepsilon_{t-j} \\
& =\sum_{j=0}^{\infty} \Psi_{j} u_{t-j},
\end{aligned}
$$

where $\Psi_{0}=W, \Psi_{j}=\Theta_{j} W$ and $u_{t}=W^{-1} \varepsilon_{t}, W$ obtained from the decomposition $\Sigma=$ $W W^{\prime}$. This implies orthonormality of the innovations, $E u_{t} u_{t}^{\prime}=I$. Observe that the decomposition of $\Sigma$ is not unique, many choices are possible, but to exploit the structure of the covariances as defined in the previous section we choose $W$ to be lower triangular with strictly positive elements on the main diagonal (Choleski decomposition). We note that this choice makes the decomposition unique, see Hamilton (1994) section 4.4.

Of course we only have estimates of the coefficients of the VAR and their asymptotic distribution and need also the asymptotic distribution of the impulse responses. These can be obtained by applying the following results obtained by Baillie (1987). These results are rather general, in the sense that we do not obtain explicit analytical formulas. In the VARMA case explicit analytical results can be derived and are given by Litkepohl (1989). We will discuss these latter results later on but state here a general way of obtaining impulse responses for a VAR process.

The results are based on the following. Suppose that the VAR $y_{t}$ depends on a finite number of parameters collected in the parameter $\alpha$, and that the limiting distribution of 
the estimator for $\alpha$ is given by

$$
\sqrt{T}(\alpha-\alpha) \sim N(0, V)
$$

where $T$ is the number of sample observations. Then it is clear that $\theta_{j}=v e c\left(\theta_{j}\right)$ is a non-linear function of the parameters $\alpha$

$$
\theta_{j}=f(\alpha)
$$

A first-order approximation of the distribution of the estimated $\hat{\theta}_{j}=f(\hat{\alpha})$ is given by

$$
\sqrt{T}\left(\hat{\theta}_{j}-\theta_{j}\right) \sim N\left(0_{n} \Delta_{j} V \Delta_{j}^{\prime}\right)
$$

where

$$
\Delta_{j}=\frac{\partial \theta_{j}}{\partial \alpha^{\prime \prime}}
$$

In case we are estimating an unrestricted VAR and are interested in the non orthogonalized. impulse responses as above, we have, see for instance Baillie (1987) or Lütkepohl (1989),

$$
\Delta_{j}=\frac{\partial v e c\left(\Theta_{j}\right)}{\partial \alpha^{\prime}}=\sum_{m=0}^{j-1} J\left(\Phi^{\prime}\right)^{j-1-m} \otimes \Theta_{m} .
$$

Here $\alpha=\operatorname{vec}\left(\left[\Phi_{1}, \ldots, \Phi_{p}\right]\right), J=\left[I_{k}, 0, \ldots, 0\right]$ with dimension $k \times k p$, and the companion matrix $\Phi$ is given by

$$
\Phi=\left[\begin{array}{cccc}
\Phi_{1} & \Phi_{2} & & \Phi_{p} \\
I_{k} & 0 & . & 0 \\
. & & & . \\
0 & . & I_{k} & 0
\end{array}\right]
$$

We stated here the results for the coefficients individual lag matrix, we could have also discussed the simultaneous distribution of the lag matrices. These results however can be easily derived from the above results, see for instance Lütkepohl (1989).

In case we are interested in orthogonalized impulse responses it is a little more complicated as we also have to estimate $W$. We start from the distribution of the estimators of the VAR model. Let $\alpha$ be the vector of parameters from the lag matrices, let $\sigma=v e c h(\Sigma)$ and suppose that we have estimators $\hat{\alpha}$ and $\hat{\sigma}$ such that the following holds:

$$
\sqrt{T}\left(\begin{array}{c}
\hat{\alpha}-\alpha \\
\hat{\sigma}-\sigma
\end{array}\right) \sim N\left(0,\left[\begin{array}{cc}
\Sigma_{(\alpha)} & 0 \\
0 & \Sigma_{(\sigma)}
\end{array}\right]\right)
$$

then we obtain (Lütkepohl, (1991))

$$
\sqrt{T}\left(\hat{\psi}_{j}-\psi_{j}\right) \sim N\left(0, C_{j} \Sigma_{(\alpha)} C_{j}^{I}+\bar{C}_{j} \Sigma_{(\sigma)} \bar{C}_{j}^{\prime}\right)
$$

where $\psi_{j}=\operatorname{vec}\left(\Psi_{j}\right), C_{0}=0, C_{j}=\frac{\partial \operatorname{vec}\left(\theta_{j} W\right)}{\partial \alpha^{\prime}}=\left(W^{\prime} \otimes I\right) \frac{\partial \operatorname{vec}\left(\theta_{j}\right)}{\partial \alpha^{\prime}}=\left(W^{\prime} \otimes I\right) \Delta_{j}$, and $\bar{C}_{j}=\frac{\partial \operatorname{vec}\left(\Psi_{j}\right)}{\partial \sigma^{\prime}}=\frac{\partial \operatorname{vec}\left(\Theta_{j} W^{\prime}\right)}{\partial \sigma^{\prime}}=\left(I \otimes \Theta_{j}\right) \frac{\partial \operatorname{vec}(W)}{\partial \sigma^{\prime}}=\left(I \otimes \Theta_{j}\right) H$, where $H=\frac{\partial \operatorname{vec}(W)}{\partial \sigma^{\prime}}=$ $L_{k}^{\prime}\left[L_{k}\left(I+K_{k k}\right)(W \otimes I) L_{k}^{\prime}\right]^{-1}$. For an explanation of the known matrices $L_{k}, K_{k k}$ we refer to the appendix. For an introduction into time series analysis especially Vector Autore gressions and Impulse Response Analysis, we refer to Hamilton (1994) and Lütkepohl (1991). 


\subsection{Estimation and a symptotics}

In this section we consider estimation and asymptotics in the different models introduced in section two. We start by deriving the likelihood function of the general model which in subsection two we specialize to the different parametrizations. In the third subsection a least squares estimation techrique is presented. Finally we give in subsection four the asymptotic distributions for the estimators of the parameters in the models with the different variance structures.

\subsubsection{Maximum likelihood}

To analyze the four models defined by (4.1)-(4.6) we introduce a slightly more general model in the sense that we assume the contemporaneous covariance matrix to depend on the general vector $\theta$. The $N$ uncorrelated $k$ dimensional stationary vector autoregressive processes of order $p$ are given by

$$
\left(y_{i, t}-\mu_{i}\right)=\Phi_{1}\left(y_{i, t-1}-\mu_{i}\right)+\ldots+\Phi_{p}\left(y_{i, t-p}-\mu_{i}\right)+\varepsilon_{i, t},
$$

where $\varepsilon_{i t} \sim N\left(0, \Omega_{i}(\theta)\right)$, and $\theta$ is an $m$ dimensional vector. The covariance matrix is assumed to be a twice differentiable function of $\theta$. Suppose we have observed data $y_{i,-p+1}, \ldots, y_{i, 1}, \ldots y_{i, T}$ for $i=1, \ldots, N$. Defining $\Phi=\left[\Phi_{1}, \ldots, \Phi_{p}\right], \alpha=v e c(\Phi), \mu=\left(\mu_{1}^{\prime}, \ldots, \mu_{N}^{\prime}\right)^{\prime}$, $X_{i, t}=\left(y_{i, t-1}-\mu_{i}, . ., y_{i, t-p}-\mu_{i}\right)^{\prime}, x_{i, t}=\left(y_{i, t-1}, . ., y_{i, t-p}\right)^{t}$ and $Y_{i, t}=y_{i, t}-\mu_{i}$ the likelihood function conditional on the initial observations $y_{i,-p+1, \cdots,}, y_{i, 0}$, of $(4.29)$ is given by

$$
\begin{aligned}
L= & -\frac{N T k}{2} \log (2 \pi)+\frac{T}{2} \sum_{i=1}^{N} \log \left(\left|\Omega_{i}\right|^{-1}\right) \\
& -\frac{1}{2} \sum_{i=1}^{N} \sum_{t=1}^{T}\left(Y_{i, t}-\Phi X_{i, t}\right)^{\prime} \Omega_{i}^{-1}\left(Y_{i, t}-\Phi X_{i, t}\right) .
\end{aligned}
$$

Maximizing this likelihood function with respect to $\Phi, \theta$ and $\mu_{i}, i=1, . ., N$ leads to the following results:

Theorem 14 The maximum of the likelihood function (4.90) is given by the simultaneous solution of the following first order conditions

$$
\begin{gathered}
\hat{\mu_{i}}=\frac{1}{T}\left[\left(I-\hat{\Phi}_{1}-\ldots-\hat{\Phi}_{p}\right)\right]^{-1} \sum_{i=1}^{T}\left(y_{i, t}-\hat{\Phi} x_{i, t}\right), \quad i=1, \ldots, N, \\
\hat{\alpha}=\left[\sum_{i=1}^{N} \sum_{t=1}^{T}\left(\bar{X}_{i, t} \bar{X}_{i, t}^{\prime} \otimes \hat{\Omega}_{i}^{-1}\right)\right]^{-1} \sum_{i=1}^{N} \sum_{t=1}^{T}\left(\bar{X}_{i, t} \otimes \hat{\Omega}_{i}^{-1}\right) \bar{Y}_{i, t}
\end{gathered}
$$

and

$$
\frac{1}{2} \sum_{i=1}^{N} \sum_{t=1}^{T}\left(\operatorname{vec}\left(\hat{\Omega}_{i}^{-1}-\hat{\Omega}_{i}^{-1} u_{i, t} u_{i, t}^{\prime} \hat{\Omega}_{i}^{-1}\right)\right)^{\prime} \frac{\partial \operatorname{vec}\left(\hat{\Omega}_{i}\right)}{\partial \theta^{\prime}}=0,
$$

where $\bar{X}_{i, t}$ and $\bar{Y}_{i, t}$ are $X_{i, t}$ and $Y_{i, t}$ with $\mu_{i}$ replaced by $\hat{\mu}_{i}$. 
Proof. The proof of the Theorem is given in the Appendix.

Observe that the estimator for the individual effects, or individual means, has the same structure as the estimator of the same individual means using only the individual time series under consideration. However the resulting estimates are not the same. This is due to the fact that in our pooled model the coefficients of the lag polynomial are estimated using the information of the other time series, in contrast to the estimator based on the individual time series alone. Asymptotically however there is no efficiency gain for the estimation of the individual mean. This follows directly from Theorem 15 below.

The estimator for the parameters in the lag polynomial does, in contrast to the ML estimator based on the individual time series, depend on the different individual contemporaneous covariance matrices.

Another important result is that the estimators for the individual effects and the lag polynomial parameters conditional on the parameter $\theta$, or equivalently, $\Omega_{i}$ are the same for each model. The only difference between the estimators of the different models is the way the restrictions are imposed on the parameters of the individual covariance matrices and the resulting estimate for $\Omega_{i}$.

The next theorem gives the asymptotic distribution of the ML estimators of the parameters $\mu_{i}, \Phi$, and $\theta$.

Theorem 15 Assume that each of the individual time series described by (4.29) is stationary. Then the asymptotic distribution of the Maximum Likelihood estimators as given by Theorem 14 is given by

$$
\left(\begin{array}{lll}
\sqrt{T} & & \\
& \sqrt{N T} & \\
& & \sqrt{N T}
\end{array}\right)\left(\begin{array}{c}
\hat{\mu}-\mu \\
\hat{\alpha}-\alpha \\
\hat{\theta}-\theta
\end{array}\right) \longrightarrow N\left(0,\left(\begin{array}{lll}
I_{(\mu)}^{-1} & 0 & 0 \\
0 & I_{(\alpha)}^{-1} & 0 \\
0 & 0 & I_{(\theta)}^{-1}
\end{array}\right)\right)
$$

with

$$
\begin{array}{r}
I_{[\mu)}=\operatorname{diag}\left(\Upsilon^{\prime} \Omega_{1}^{-1} \Upsilon, \ldots, \Upsilon^{\prime} \Omega_{N}^{-1} \Upsilon\right), \\
I_{(\alpha)}=\frac{1}{N} \sum_{i=1}^{N}\left(\Gamma^{i}(p) \otimes \Omega_{i}^{-1}\right), \\
I_{(\theta)}=\frac{1}{2 N} \sum_{i=1}^{N} \frac{\partial v e c\left(\Omega_{i}\right)^{\prime}}{\partial \theta} K_{k, k}\left[\Omega_{i}^{-1} \otimes \Omega_{i}^{-1}\right] \frac{\partial v e c\left(\Omega_{i}\right)}{\partial \theta^{\prime}} .
\end{array}
$$

where $\Upsilon=\left(I-\Phi_{1}-\ldots-\Phi_{p}\right)$ and $\Gamma^{i}(p)=E\left(X_{i, t} X_{i, t}^{\prime}\right)$. The Commutation matrix $K_{m_{0} n}$ is the matrix with the property that for any $(m \times n)$ matrix $A$ the relation $K_{m, n} \operatorname{vec}(A)=\operatorname{vec}\left(A^{\prime}\right)$ holds.

Proof. The consistency of the estimators follows directly from the finiteness of $N$ together with the abservation that a system of $N$ independent stationary VAR's can be viewed as one large VAR with a finite number of parameters with restrictions. Therefore the conventional maximum likelihood results hold. However as we are considering a particular 
set of restrictions to hold we get some specific results. The proof of the Theorem is completed in the appendix where we derive the asymptotic information matrix.

The models described in section 2 are all special cases of the model analyzed in this section. It is easily checked that we only have to specify the appropriate vector $\theta$ to describe any of the four models in section 2. Observe that the parametrization of the variance only affects the asymptotic distribution of the estimators of the parameters of the variance matrix, $\theta$, i.e. $I_{(\theta)}$.

Further we observe the important result that asymptotically the distribution of the estimators of the individual effects, parameters of the lag polynomial and the parameters in the covariance matrices are independent of each other. Therefore to derive the asymptotic distribution of the estimators of all parameters simultaneously it is sufficient to derive the distribution for the parameters in the different covariance structures. The asymptotic distribution of the individual effects and parameters in the lag matrices are given by the above theorem and putting these marginal distributions together yields the simultaneous asymptotic distribution of the estimators.

In the next two subsections we will specialize the above theorems by choosing specific vectors $\theta$.

\subsubsection{The $\mathrm{ML}$ estimators}

As the discussion after Theorem 14 points out the estimators for the individual means and the pooled lag polynomial conditional on the parameter $\theta$ are given by (4.31) and (4.32). The first order conditions for the parameters $\theta$ are given by (4.33). These conditions have to be adapted to the different models.

Before defining the appropriate parameters of $\theta$ we introduce the following two operators on square matrices: The $w(\bullet)$ operator which takes out the diagonal elements of a matrix i.e. $w\left(\Sigma_{i}\right)=\left(\sigma_{1}^{(i)}, \ldots, \sigma_{k}^{(i)}\right)^{\prime}$, and the $v(\bullet)$ operator which selects the elements below the main diagonal, i.e. $v(A)=\left(a_{2,1}, \ldots, a_{2, k}, a_{3,2,}, \ldots, a_{3, k}, \ldots, a_{k, k}\right)^{\prime}$. Using these operators we can state the correct vectors for the different covariance structures. For the covariance structure $\Omega^{p}$ we have $\theta^{p}=\operatorname{vech}(\Omega)$, for $\Omega^{i}$ we have $\theta^{i}=\left(\operatorname{vech}\left(\Omega_{1}\right)^{\prime} \text {, ..vech }\left(\Omega_{N}\right)^{\prime}\right)^{\prime}$. The reparametrization of $\Omega^{s b}$ implies $\theta^{s b}=\left(v(\Gamma)^{\prime}, w\left(\Sigma_{1}\right)^{\prime}, \ldots w\left(\Sigma_{N}\right)^{\prime}\right)^{\prime}$ and finally the parameter vector for $\Omega^{s w}$ is given by a vector with the same structure $\theta^{s t w}=\left(v(\Gamma)^{\prime}, w\left(\Sigma_{1}\right)^{\prime}, \ldots w\left(\Sigma_{N}\right)^{\prime}\right)^{\prime}$.

Theorem 16 Define $u_{i, t}=y_{i, t}-\mu_{i}-\Phi X_{i, t}$, then the estimators for the parameters in the models defined by equations (4.1)-(4.5) are given by (4.31) and (4.32) and

$$
\hat{\theta^{p}}=\frac{1}{N T} \sum_{i=1}^{N} \sum_{t=1}^{T} \operatorname{vech}\left(u_{i, t} u_{i, t}^{t}\right)
$$

and

$$
\hat{\theta^{i}}=\left(\frac{1}{T} \sum_{t=1}^{T} \operatorname{vech}\left(u_{1, t} u_{1, t}^{t}\right)^{\prime}, \ldots, \frac{1}{T} \sum_{t=1}^{T} \operatorname{vech}\left(u_{N, t} u_{N, \ell}^{\prime}\right)^{\prime}\right)^{\prime}
$$

for the models with covariance structures $\Omega^{i}$ and $\Omega^{p}$ respectively. For the model with covariance structure $\Omega^{s b}$, the estimator for the parameters $\theta^{\text {st }}$ is giver by the simultaneous 
solution of (4.31) and (4.32) and

$$
\begin{gathered}
\Psi_{k} \operatorname{vec}\left(\Sigma_{i}^{2}\right)=\frac{1}{T} \sum_{t=1}^{T} \Psi_{k} \operatorname{vec}\left(\Gamma^{-1} u_{i, t} u_{i, t}^{\prime} \Gamma^{-1 \prime}\right), \quad i=2, \ldots N, \\
\tilde{L}_{k} \operatorname{vec}\left(I_{k}\right)=\frac{1}{N T} \sum_{i=1}^{N} \sum_{t=1}^{T} \tilde{L}_{k} \operatorname{vec}\left(\Gamma^{-1 \prime} \Sigma_{i}^{-2} \Gamma^{-1} u_{i, t} u_{i, t}^{\prime}\right) .
\end{gathered}
$$

For $\Omega^{\text {sw }}$, the estimator $\hat{\theta^{\text {sw }}}$ is given by the simultaneous solution of (4.31) and (4.32) and

$$
\begin{array}{r}
\Psi_{k} \operatorname{vec}\left(I_{k}\right)=\frac{1}{T} \sum_{i=1}^{N} \sum_{t=1}^{T} \Psi_{k} \operatorname{vec}\left(\Sigma_{i}^{-1} \Gamma^{\prime-1} \Gamma^{-1} \Sigma_{i}^{-1} u_{i, t} u_{i, t}^{\prime}\right), \quad i=2, \ldots N, \\
\tilde{L}_{k} \operatorname{vec}\left(\Gamma \Gamma^{\prime}\right)=\frac{1}{N T} \sum_{i=1}^{N} \sum_{t=1}^{T} \widetilde{L}_{k} \operatorname{vec}\left(\Sigma_{i}^{-1} u_{i, t} u_{i, t}^{\prime} \Sigma_{i}^{-1}\right) .
\end{array}
$$

The matrix $\Psi_{k}$ is the matrix with the property that vec $(A)=\Psi_{k}^{\prime} w(A)$ for every diagonal $(k \times k)$ matrix $A$. The matrix $\widetilde{L}_{k}$ is the matrix with the property that vec $(A)=\widetilde{L}_{k} v(A)$ for every strictly lower triangular matrix $A$. For more on these matrices we refer to the appendix.

Proof. The derivation of the first order conditions is given in the appendix.

Solving all the first order conditions requires iteration until convergence. In the next section however we introduce a Generalized Least Squares method which is asymptotically efficient after two steps.

\subsubsection{GLS estimators}

To obtain estimates for the individual means $\mu_{i}$ one can also use the usual estimator of the mean $\hat{\mu}_{i}=\frac{1}{T} \sum_{i=1}^{N} y_{i, t}, i=1, \ldots, N$. It is well known, see for instance Lutkepohl (1991), that the asymptotic properties of this estimator are the same as those derived for the ML estimator (4.31). The asymptotic distribution derived in Theorem 15 characterizes these properties. This estimator is mathematically the same for all models in contrast to the maximum likelihood estimator given in (4.31).

Given the estimates $\hat{\mu}_{i}$ the GLS estimator of the parameters of the lag matrices $\alpha=$ $\operatorname{vec}\left(\left[\Phi_{1}, \ldots, \Phi_{p}\right]\right)$ becomes

$$
\hat{\alpha}=\left[\sum_{t=1}^{T} \sum_{i=1}^{N}\left(\tilde{X}_{i, t} \tilde{X}_{i, t}^{\prime} \otimes\left(\Omega_{i}\right)^{-1}\right)\right]^{-1} \sum_{t=1}^{T} \sum_{i=1}^{N}\left(\tilde{X}_{i, t} \otimes\left(\Omega_{i}\right)^{-1}\right)\left(y_{i, t}-\hat{\mu}_{i}\right),
$$

where $\tilde{X}_{i, t}=\left(\left(y_{i, t-1}-\hat{\mu}_{i}\right)^{\prime}, \ldots,\left(y_{i, t-p}-\hat{\mu}_{i}\right)^{\prime}\right)^{\prime}$.

This estimator is the equal to the ML estimator (4.32) given in Theorem 14, and depends on the covariance matrices $\Omega_{i}$ and is therefore different for every different covariance structure. 
To make this estimator feasible we need an estimator for the covariance matrices $\Omega_{i}$, taking into account the different structures of the different models. Therefore we propose the following procedure. In the first step estimate the Iag coefficients by

$$
\hat{\alpha}=\left[\sum_{t=1}^{T} \sum_{i=1}^{N}\left(X_{i, t} X_{i, t}^{i} \otimes I\right)\right]^{-1} \sum_{t=1}^{T} \sum_{i=1}^{N}\left(X_{i, t} \otimes I\right)\left(y_{i, t}-\hat{\mu}_{i}\right)
$$

Then use this estimate to obtain estimates for the individual covariance matrix

$$
\hat{\Omega}_{i}=\frac{1}{T} \sum_{t=1}^{T} u_{i, t} u_{i, t}^{\prime}, \quad i=1, \ldots, N
$$

where $u_{i, t}$ are the residuals using the estimates (4.35). Then:

- For the model with covariance structure $\Omega^{p}$ use for each of the individual covariances $\Omega_{i}$ in (4.34)

$$
\hat{\Omega}^{p}=\frac{1}{N} \sum_{i=1}^{N} \hat{\Omega}_{i}
$$

- For the model with covariance structure $\Omega^{i}$ simply use $\hat{\Omega}_{i}$ given by (4.36) as estimator for the individual covariance matrix $\Omega_{i}$ in (4.34).

- For the covariance structure $\Omega^{s b}$ use

$$
\hat{\Omega}_{i}=\hat{\Gamma} \hat{\Sigma_{i}} \hat{\Sigma}_{i} \hat{\Lambda}^{\prime}
$$

in (4.34). For the construction of the estimates $\hat{\Gamma}$ and $\hat{\Sigma}_{i}$ we observe first that if we make a Choleski decomposition of $\Omega_{i}=L_{i} L_{i}^{\prime}$ we obtain $L_{i}=\Gamma \Sigma_{i}$, i.e.

$$
\Gamma=\left[\begin{array}{llll}
1 & & & \\
\gamma_{2,1} & 1 & & \\
& & & \\
\gamma_{k_{1} 1} & \gamma_{k, 2} & \gamma_{k, k-1} & 1
\end{array}\right], \quad L_{i}=\left[\begin{array}{lll}
\sigma_{i, 1} & & \\
\sigma_{i, 1} \gamma_{2,1} & \sigma_{i, 2} & \\
& & \\
\sigma_{i, 1} \gamma_{k, 1} & \sigma_{i, 2} \gamma_{k, 2} & \sigma_{i, k}
\end{array}\right] \text {. }
$$

so the $\sigma_{i, j}$ are given by the elements on the main diagonal of $L_{i}$. Using this observation we can easily obtain estimates $\hat{\sigma}_{i, j}$ by making the Choleski decomposition of $\hat{\Omega}_{i}=\hat{L}_{i} \hat{L}_{i}^{\prime}$ and obtaining the main diagonal elements. The estimate for $\Gamma$ can then be obtained by

$$
\hat{\Gamma}=\frac{1}{N} \sum_{i=1}^{N} \hat{L}_{i}\left(\hat{\Sigma}_{i}\right)^{-1}
$$

- For the covariance structure $\Omega^{s w}$ use

$$
\hat{\Omega}_{i}=\hat{\Sigma}_{i} \hat{\Gamma} \hat{\Gamma}^{\prime} \hat{\Sigma}_{i}
$$


in (4.34). The estimates $\hat{\Gamma}$ and $\hat{\Sigma}_{i}$ are obtained in a similar manner as in the structure $\Omega^{2 b}$. We have by the Choleski decomposition of $\Omega_{i}=L_{i} L_{i}^{\prime}$ and $L_{i}=\Sigma_{i} \Gamma$

$$
\Gamma=\left[\begin{array}{lll}
1 & & \\
\gamma_{2,1} & 1
\end{array}\right], \quad L_{i}=\left[\begin{array}{lll}
\sigma_{i, 1} & & \\
\sigma_{i, 2} \gamma_{2,1} & \sigma_{i, 2} & \\
& & \\
\gamma_{k, 1} & \gamma_{k, 2} & 1
\end{array}\right] .
$$

so the $\sigma_{i, j}$ are given by the elements on the main diagoral of $L_{i}$. Using this we can easily obtain estimates $\hat{\sigma}_{i, j}$ by making the Choleski decomposition of $\hat{\Omega}_{i}=\hat{L}_{i} \hat{L}_{i}^{\prime}$ and obtaining the main diagonal elements. The estimate for $\Gamma$ can then be obtained by

$$
\hat{\Gamma}=\frac{1}{N} \sum_{i=1}^{N}\left(\hat{\Sigma}_{i}\right)^{-1} \hat{L_{i}} .
$$

Using the estimates for $\Omega_{i}$ in (4.34) we can reestimate the lag matrices parameters $\alpha$ and iterate the procedure until convergence. Observe that all estimators are consistent in each step. We can iterate this process until convergence but after one iteration the estimator is asymptotically equivalent to MLE and therefore efficient as the initial estimates are consistent and asymptotically normally distributed. The exact conditions under which two-step estimators based on the first order conditions of the ML estimator are efficient are given in the literature, amongst others Magnus (1978).

The use of this estimator is suggested when performing simulations or using bootstrap confidence bounds. The number of iterations can be limited by the researcher while asymptotic efficiency is guaranteed. The number of iterations in the straightforward MLE cannot be controlled by the researcher. So the practical use of this estimator is limited to the case where one uses the explicitly derived asymptotic distributions.

\subsubsection{Asymptotics}

In this section we derive the asymptotic distributions of the ML estimators as given in Theorem 16. As the Least Squares estimators are asymptotically equivallent to the ML estimators it follows that their asymptotic distributions are also the same. Therefore we do not have to discuss the asymptotics of the LS estimators separately.

As pointed out earlier Theorem 16 implies that the covariance matrix is block diagonal. This means that we can study the asymptotic behavior of estimators of the individual means, the lag polynomial parameters and the variance parameters separately. The com variance matrices of the individual means and the lag polymomial parameters have the same structure for each of the different models, only the appropriate individual covariance matrix has to be used in Theorem 15 to obtain the asymptotic distributions. The information matrix associated with the estimates for the variances however is different for each model. We have to study them case by case. The following theorem gives the asymptotic distributions of the estimators of the parameters in the different covariance structures. 
Before we state the theorem we introduce some additional notation. Define $\gamma=v(\Gamma)$ and $\sigma=\left(w\left(\Sigma_{1}\right)^{\prime}, \ldots, w\left(\Sigma_{N}\right)^{\prime}\right)^{\prime}$ then the partitioned information matrix for the covariance structures $\Omega^{s w}$ and $\Omega^{s b}$ is given by

$$
I_{(\theta)}=\left[\begin{array}{ll}
I_{\gamma \gamma^{\prime}} & I_{\gamma \sigma^{\prime}} \\
I_{\sigma \gamma^{\prime}} & I_{\sigma \sigma^{\prime}}
\end{array}\right]
$$

In the theorem we also make use of some so called L-structured matrices, see Magnus (1988). We introduced some of them already in previous theorems but we recall their main properties here. For more on this class of matrices we refer to the appendix.

L-structured matrices are real matrices of a certain dimension and order that satisfy a given set of linear restrictions. We use them to handle the restrictions on the covariance matrices of the innovations. The Duplication matrix $D_{k}$ is defined as the matrix for which the relation

$$
\operatorname{vec}(A)=D_{k} \operatorname{vech}(A)
$$

holds for every symmetric $(k \times k)$ matrix $A$. The Commutation matrix $K_{n, m}$ is the matrix with the property that for any $(n \times m)$ matrix $A$ the relation

$$
K_{n, m} \operatorname{vec}(A)=\operatorname{vec}\left(A^{\prime \prime}\right)
$$

holds. The matrix $\Psi_{k}$ is the matrix with the property that

$$
\operatorname{vec}(A)=\Psi_{k}^{\prime} w(A)
$$

for every diagonal $(k \times k)$ matrix $A$. The matrix $\widetilde{L}_{k}$ is the matrix with the property that

$$
\operatorname{vec}(A)=\hat{L}_{k}^{\prime} v(A)
$$

for every strictly lower triangular matrix $A$. We now state the theorem.

Theorem 17 We have, as $T \rightarrow \infty$,

$$
\sqrt{N T}(\hat{\theta}-\theta) \rightarrow N\left(0, I_{(\theta)}^{-1}\right)
$$

The Fisher Information matrix $I_{(\theta)}$ is given by the following equations. When $\theta=\theta^{p}$

$$
I_{(\theta \mathrm{P})}=\frac{1}{2} D_{k}^{\prime}\left(\Omega^{-1} \otimes \Omega^{-1}\right) D_{k}
$$

when $\theta=\theta^{i}$

$$
I_{\left(\theta^{i}\right)}=\frac{1}{2 N} \sum_{i=1}^{N}\left(e_{i, N} e_{i, N}^{i} \otimes D_{k}^{i}\left(\Omega_{i}^{-1} \otimes \Omega_{i}^{-1}\right) D_{k}\right),
$$

where $e_{i, N}$ is an $N \times 1$ vector of zeros with a 1 at the $i$-th position. For $\theta=\theta^{\text {sb }}$ we have, using the partitioning given by (4.39),

$$
\begin{aligned}
& I_{\gamma \gamma^{\prime}}=\frac{1}{N} \sum_{i=1}^{N} \tilde{L}_{K}\left(\left(\Sigma_{i}^{2} \otimes \Omega_{i}^{-1}\right)+\left(\Gamma^{-1} \otimes \Gamma^{-1 \gamma}\right) K_{k k}\right) \tilde{L}_{k}^{\prime}, \\
& I_{\sigma \gamma^{\prime}}=\frac{1}{N} \sum_{i=1}^{N}\left(e_{i, N} \otimes \Psi_{k}\left(\left(\Gamma^{\prime} \otimes \Sigma_{i}^{-1} \Gamma^{-1}\right)+\left(\Sigma_{i}^{-1} \Gamma^{-1} \otimes \Sigma_{i}\right) K_{k k}\right) \tilde{L}_{k}^{\prime}\right), \\
& I_{\sigma \sigma^{\prime}}=\frac{1}{N} \sum_{i=1}^{N}\left(e_{i, N} e_{i, N}^{\prime} \otimes \Psi_{k}\left(\left(\Sigma_{i}^{-2} \otimes I_{k}\right)+\left(\Sigma_{i} \otimes \Sigma_{i}^{-1}\right) K_{k k k}\right) \Psi_{k}^{\prime}\right) .
\end{aligned}
$$


For $\theta=\theta^{\text {sw }}$ we have, using the same partitioning (4.39),

$$
\begin{aligned}
& I_{\gamma \gamma^{\prime}}=\tilde{L}_{K}\left(\left(I \otimes \Gamma^{-1 \prime} \Gamma^{-1}\right)+\left(\Gamma^{-1} \otimes \Gamma^{-1 \prime}\right) K_{k k}\right) \tilde{L}_{k}^{\prime}, \\
& I_{\sigma \gamma^{\prime}}=\frac{1}{N} \sum_{i=1}^{N}\left(e_{i, N} \otimes \Psi_{k}\left(\left(\Gamma \otimes \Sigma_{i}^{-1} \Gamma^{-1 /} \Gamma^{-1}\right)+\left(I_{k} \otimes \Sigma_{i}^{-1} \Gamma^{-1 \prime}\right) K_{k k}\right) \tilde{L}_{k}^{\prime}\right), \\
& I_{\sigma \sigma^{\prime}}=\frac{1}{N} \sum_{i=1}^{N}\left(e_{i, N} e_{i, N}^{\prime} \otimes \Psi_{k}\left(\left(\Gamma \Gamma^{\prime} \otimes \Omega_{i}^{-1}\right)+\left(\Sigma_{i}^{-1} \otimes \Sigma_{i}^{-1}\right) K_{k k}\right) \Psi_{k}^{\prime}\right) .
\end{aligned}
$$

Observe that the estimators for the parameters of $\theta^{i}$ are asymptotically independent for each individual. This means that asymptotically we do not gain additional efficiency for the estimation of the individual covariance matrix due to pooling the lag matrices.

We can rewrite this Theorem in a number of ways. For instance we could give the covariances explicitly. However we leave the theorem in this form because it can be implemented in matrix packages such as Gauss or Matlab in a straightforward manner. Observe also that although the information matrix has a nice structure, the covariance, which is the inverse of the information matrix, does not have this nice structure: every parameter is correlated with all the others.

\subsection{Testing}

As we have introduced restrictions on the contemporaneous covariance matrix of the individuals and cross individual restrictions on the lag matrices it is a good statistical practice to check whether these restrictions indeed hold. To test for these restrictions we use the likelihood ratio test.

The likelihood ratio statistic is of the form

$$
\lambda_{L R}=2\left(\log \left(l\left(\hat{\delta}_{u}\right)\right)-\log \left(l\left(\hat{\delta}_{r}\right)\right)\right)
$$

where $\hat{\delta}_{u}$ is the unrestricted and $\hat{\delta}_{\mathrm{r}}$ the restricted maximizer of the likelihood function $l(\bullet)$. We assurne that the restricted parameter vector $\delta_{r}(k \times 1)$ is a (non)linear function of the unrestricted parameter vector $\delta_{u}(r \times 1), k<r$

$$
m\left(\delta_{u}\right)=\delta_{r}
$$

It is assumed that the function $m(\bullet)$ is a smooth function in a neighborhood of its true value. Asymptotically under $H_{0}: m\left(\hat{\delta}_{*}\right)=\hat{\delta}_{\tau}$ and the likelihood ratio statistic $\lambda_{L R}$ is chi-square distributed with $(r-k)$ degrees of freedom:

$$
\lambda_{L R} \sim \chi^{2}(r-k) .
$$

Since the function $m$ is smooth by assumption we will use this result to test the restrictions discussed in section 4.2 . 
To identify a model we have to perform several tests. First we have to determine the order of the VAR. Then after having determined the order of the VAR we have to test the cross individual pooling restrictions on the lag matrices and thereafter we can test for the structure of the covariances of the individuals.

For the order determination of the system we can use a sequence of likelihood ratio tests on the significance of the highest order modelled matrix of the lag polynomial. For a description of this procedure we refer the reader to Lutkepohl (1991). Instead of using the likelihood ratio we can also use other model selection criteria like Akaike's AIC (1973) and (1974), Hannan and Quinn's HQ (1979) and Quinn (1980), or the SC introduced by Schwarz (1978).

When we are interested in comparing the different covariance structures we have to realize that they are not nested. We cannot test the model $\Omega^{s w}$ against $\Omega^{s b}$ or the other way around using procedures for nested tests. We can only test them against the models $\Omega^{i}$ and $\Omega^{p}$ and the completely unrestricted models of the same order. An alternative would be to adopt a test procedure for nonnested hypotheses.

\subsection{Impulse response analysis}

In this section we give some results concerning impulse response analysis for the two models above. For more details on impulse response functions and asymptotic distributions we refer to Sims (1980) who introduced impulse response analysis; Bailly (1987) who introduced a way to calculate numerically in a general framework the asymptotic distribution of impulse response functions; Lütkepohl (1989), (1990) who derived explicit analytical results for impulse response functions in vector autoregressive processes and gives a very good introduction to multiple time series analysis in (1991). Finally, Mittnik and Zadrosky (1993) generalize many of the mentioned results. Also of interest is Giannini (1992) who considers structural VAR's.

\subsubsection{Nonorthogonalized impulse responses}

The fact that the nonorthogonalized impulse responses do not depend on the estimated covariances makes it easy to derive the impulse responses. Let $\theta_{i, j}, j=1,2, \ldots$, denote the lag matrices, for individual $i$, of the MA representation with nonorthogonalized innovations of the VAR models defined in section 2. Observe that these MA representations have the same MA matrices for all models. This implies that the nonorthogonalized impulse responses are given by the same function for each individual. Only the asymptotic distribution of the estimates of the parameters depends on the covariance structure.

Using the results from section 4.3 we directly obtain the following result.

Theorem 18 When Theorem 15 holds we have for each of the models defined in section 4.2 as $T \rightarrow \infty$

$$
\sqrt{N T} \operatorname{vec}\left(\hat{\Theta}_{i, j}-\Theta_{i, j}\right) \rightarrow N\left(0, \Delta_{j}\left(\frac{1}{N} \sum_{i=1}^{N}\left(\Gamma_{Y}^{i}(0) \otimes \Omega_{i}^{-1}\right)\right)^{-1} \Delta_{j}^{\prime}\right) .
$$


$j=1,2, . .$, The matrices $\Omega_{i}$ are the individual covariance matrices corresponding to the models with covariance structures $\Omega^{P}, \Omega^{i}, \Omega^{s b}$ and $\Omega^{s w}$ respectively. Further

$$
\Delta_{j}=\frac{\partial \operatorname{vec}\left(\Theta_{j}\right)}{\partial \operatorname{vec}(\Phi)^{\prime}}=\sum_{m=0}^{j-1} J\left(C(\Phi)^{\prime}\right)^{j-1-m} \otimes \Theta_{m},
$$

where $C(\Phi)$ denates the companion matrix as given by (4.26) and $\theta_{0}=I_{K}$.

Proof. The result follows directly from the discussion in section three.

As we can see the covariance structures for all models are similar. This result is due to the asymptotic independence of the estimators for the coefficients of the lag polynomial and the parameters of the covariance matrices.

Observe that the efficiency gain due to pooling as compared to the impulse response based on the individual observations is larger when the contemporaneous covariance matrix of the individual is small compared to the individual with the largest magnitude covariance matrix.

\subsubsection{Orthogonalized impulse responses}

Due to the orthogonalization of the covariance matrices the MA representation for the different models is not the same. We also need to take into account the asymptotic distribution of the estimated covariance parameters. This introduces some new problems. The estimates for the individual and common covariance parameters are not independent according to Theorem 17.

We have the following MA representation,

$$
\begin{aligned}
y_{i, t}-\mu & =\sum_{j=0}^{\infty} \Theta_{j} W_{i} W_{i}^{-1} \varepsilon_{i, t-j} \\
& =\sum_{j=0}^{\infty} \Psi_{j, i} u_{i, t-j}
\end{aligned}
$$

with $E\left(u_{i, t-j} u_{i, t-j}^{\prime}\right)=I_{k}$, and $\Psi_{j, i}=\Theta_{j} W_{i}$. For the models with covariance structure $\Omega^{p}, \Omega^{i}, \Omega^{s b}$ and $\Omega^{s w}$ this gives $\Psi_{j, i}=\Theta_{j} W, \Psi_{j, i}=\Theta_{j} W_{i}, \Psi_{j, i}=\Theta_{j} \Gamma \Sigma_{i}$ and $\Psi_{j, i}=\Theta_{j} \Sigma_{i} \Gamma$ respectively.

Observe that for the pooled model the impulse responses are the same for each individual. For the scaled between model, by the diagonality of $\Sigma_{i}$, the impulse response functions for the same variable differ for each individual only by a multiplicative constant corresponding to the standard deviation of the variable of the shock. For the scaled within model the impulse response function for individuals does not differ by the size of the standard deviation of the variable of the shock but by a linear combination of the individual standard deviations $\Sigma_{i}$.

Define $\psi_{i, j}=\operatorname{vec}\left(\Psi_{i, j}\right)$, then the asymptotic distribution of the orthogonalized impulse response functions is given by 
Theorem 19 When Theorem 15 holds we have for $T \rightarrow \infty$

$$
\sqrt{N T}\left(\hat{\psi}_{i, j}-\psi_{i, j}\right) \rightarrow N\left(0, C_{i, j} I_{(a)}^{-1} C_{i, j}^{\prime}+\bar{C}_{i, j}^{*} \Xi_{i}^{*} \bar{C}_{i, j}^{* \prime}\right)
$$

where $I_{(a)}^{-1}$ is given in Theorem 15. By the definition of $\Delta_{j}$ in (4.43) we have

$$
C_{i, 0}=0, \quad C_{i, j}=\left(A_{i}^{\prime} \otimes I_{k}\right) \Delta_{j}, \quad \text { for } j=1,2, \ldots
$$

where $A_{i}=W, A_{i}=W_{i}, A_{i}=\Gamma \Sigma_{i}$ and $A_{i}=\Sigma_{i} \Gamma$ for the covariance structures $\Omega^{p}, \Omega^{i}, \Omega^{s b}$ and $\Omega^{s w}$ respectively. We further have $\Xi_{i}^{p}=I_{\left(\theta^{p}\right)}^{-1}, \Xi_{i}^{i}=I_{\left(\theta^{i}\right)}^{-1} \Xi_{i}^{s b}=\left[I_{\left(\theta^{s b}\right)}^{-1}\right]_{1, i}$ and $\Xi_{i}^{s w}=$ $\left[\Gamma_{(\theta \mathrm{sw})}^{-1}\right]_{1, i}$ where $[\bullet]_{1, i}$ denotes covariances associated with the marginal distribution of $\gamma=$ $v(\Gamma)^{\prime}$ and $w\left(\Sigma_{i}\right)$. The $\bar{C}_{i, j}^{*}$ for the different models are given by

$$
\begin{array}{r}
\bar{C}_{i, j}^{p}=\left(I_{k} \otimes \Theta_{j}\right) L_{k}^{\prime}\left[L_{k}\left(I_{k^{2}}+K_{k k}\right)(W \otimes I) L_{k}^{\prime}\right]^{-1}, \\
\bar{C}_{i, j}^{i}=\left(I_{k} \otimes \Theta_{j}\right) L_{k}^{\prime}\left[L_{k}\left(I_{k^{2}}+K_{k k}\right)\left(W_{i} \otimes I\right) L_{k}^{\prime}\right]^{-1}, \\
\bar{C}_{i, j}^{s b}=\left[\left(\Sigma_{i} \otimes \Theta_{j}\right) \stackrel{\omega}{L}_{k}^{\prime}:\left(I_{k} \otimes \Gamma \Theta_{j j}\right) \Psi_{k}^{\prime}\right], \\
\bar{C}_{i, j}^{s w}=\left[\left(I_{k} \otimes \Sigma_{i} \Theta_{j}\right) \tilde{L}_{k}^{\prime}:\left(\Gamma^{\prime} \otimes \Theta_{j}\right) \Psi_{k}^{\prime}\right] .
\end{array}
$$

The information matrices are derived in Theorem 17. The theorem only states the marginal result for one individual. It can be adapted easily to the joint result for all individuals at the same time, although one is usually interested in the results for one particular individual.

Proof. This proof is based on the results presented at the end of section three. The result for the variance component associated with the lag polynomial follows directly from $C_{j}=\frac{\partial v e c\left(\theta_{j} A\right)}{\partial \alpha^{\prime}}=\left(A^{\prime} \otimes I\right) \frac{\partial v e c\left(\theta_{j}\right)}{\partial \alpha^{\prime}}=\left(A^{\prime} \otimes I\right) \Delta_{j}$. The result for the variance component associated with the parameters in the covariance matrices follows directly from $\bar{C}_{t, j}=$ $\frac{\partial v e c\left(\Psi_{i, j}\right)}{\partial \theta^{\prime}}=\frac{\partial \operatorname{vec}\left(\Theta_{j} A_{i}\right)}{\partial \theta^{\prime}}=\left(I \otimes \Theta_{j}\right) \frac{\partial v e c\left(A_{i}\right)}{\partial \theta^{\prime}}$.

We are now left with calculating $\frac{\partial v e c\left(A_{i}\right)}{\partial \theta^{\prime}}$. For the pooled covariance structure we obtain

$$
\frac{\partial v e c\left(A_{i}\right)}{\partial \theta^{r}}=\frac{\partial v e c(W)}{\partial v e c h(\Omega)^{\prime}}=L_{k}^{\prime}\left[L_{k}\left(I_{k^{2}}+K_{k k}\right)(W \otimes I) L_{k}^{\prime}\right]^{-1}
$$

see Lütkepohl (1989). For the covariance structure $\Omega^{i}$ we obtain immediately

$$
\frac{\partial \operatorname{vec}\left(A_{i}\right)}{\partial \theta^{x}}=\frac{\partial v e c\left(W_{i}\right)}{\partial v e c h\left(\Omega_{i}\right)^{\prime}}=L_{k}^{\prime}\left[L_{k}\left(I_{k^{2}}+K_{k k}\right)\left(W_{i} \otimes I\right) L_{k}^{\prime}\right]^{-1} \text {. }
$$

For the cotrariance structure $\Omega^{s b}$ we have with $\gamma=v(\Gamma)$

$$
\begin{aligned}
\frac{\partial \operatorname{vec}\left(A_{i}\right)}{\partial\left(\gamma^{\prime}, w\left(\Sigma_{i}\right)^{\prime}\right)} & =\frac{\partial v e c\left(\Gamma \Sigma_{i}\right)}{\partial\left(\gamma^{\prime}, w\left(\Sigma_{i}\right)^{\prime}\right)} \\
& =\left[\left(\Sigma_{i} \otimes I_{k}\right) \tilde{L}_{k}^{\prime}:\left(I_{k} \otimes \Gamma\right) \Psi_{k}^{\prime}\right]
\end{aligned}
$$


Finally for the covariance structure $\Omega^{s w}$ we have

$$
\begin{aligned}
\frac{\partial \operatorname{vec}\left(A_{i}\right)}{\partial\left(\gamma^{\prime}, w\left(\Sigma_{i}\right)^{\prime}\right)} & =\frac{\partial \operatorname{vec}\left(\Sigma_{i} \Gamma\right)}{\partial\left(\gamma^{\prime}, w\left(\Sigma_{i}\right)^{\prime}\right)} \\
& =\left[\left(I_{k} \otimes \Sigma_{i}\right) \stackrel{\omega}{L}_{k}^{\prime}:\left(\Gamma^{\prime} \otimes I_{k}\right) \Psi_{k}^{\prime}\right] .
\end{aligned}
$$

Combining these results gives the result as stated in the theorem.

\subsection{An application: A small macroeconomic analysis}

In this section, we present an application of the models and techniques analyzed in the preceding sections. We consider the following three key macroeconomic variables: Gross domestic product, money balances and the price level. We investigate whether the proposed models and sets of restrictions indeed are appropriate and lead to a gain in statistical power. We further investigate whether it is possible to identify the different covariance structures. For this purpose we use the same data set as in chapter three. From this data set, consisting of 18 OECD countries, we select a subset of 6 countries. We estimate and identify a trivariate model for each of these 6 countries.

\subsubsection{The empirical model}

We study the short term behavior of three key macroeconomic variables, activity measured by GDP, money and prices. An unrestricted VAR-model for these three variables can be interpreted as a subset of the recuced form of a possibly more complicated macroeconomic model.

To judge the interaction between money, prices and activity fluctuation, the VAR technology developed in the previous sections is used. The data available for each country are nominal money (M1), nominal GDP in 1980 prices and the consumer price index $(1980=100)$ collected annually from 1957 to 1990 . The data are obtained from the main International Monetary Funds International Financial Statistics Data Base. The observed period is $1957-1990$

The countries included in the model are Austria, Belgium, Germany, the Netherlands, the United Kingdom and Switzerland. We transformed the data in several ways. First we deflated the GDP with the price index and took the log difference to obtain stationary series of real growth rates. We denoted this variable by $y_{i, t}$. We transformed nominal money accordingly and denoted it by $m_{i, t}$. Finally we obtained the second order time difference of the log price index ${ }^{2}$ and denoted this variable by $p_{i, t}$.

\footnotetext{
${ }^{2}$ We started off by checking for the order of integration of the different univariate time series of all 18 countries in the data set. We found during the analysis of the price index series that we can divide the countries in the data set into two groups: A set of countries for which the price index series is I(2) and a set of countries for which it is $\mathbb{I}(1)$. For obvious reasons we cannot pool countries from both these groups. For the subsequent analysis we choose countries from the group of countries for which the price index series is I(2). We further note that this result implies that we assume the nominal GDP series also to be $1(2)$.
} 


\begin{tabular}{|l|l|l|l|}
\hline VAR order & AIC & HQ & SC \\
\hline 1 & -63.51 & -61.92 & -58.42 \\
2 & -69.43 & -67.04 & -61.79 \\
3 & -66.71 & -63.52 & -55.52 \\
\hline
\end{tabular}

Table 4.1: Results order selection criteria.

By using these transformations we obtain stationary variables which we analyze below. We note that we do not allow for possible cointegration restrictions between the variables. We also note that the nonrejection of the $\mathrm{I}(2)$-ness of the price series may be induced by time aggregation, which introduces a higher order MA process which influences the power of the used unit root tests. However, we note that if $\log$ prices are $\mathrm{I}(2)$, the inflation rate $\pi_{i, t}$ will be $\mathrm{I}(1)$. Our finding that $m_{i, t}$ and $y_{i, t}$ are $\mathrm{I}(1)$ and $p_{i, t}$ is $\mathrm{I}(2)$ is in line with the assumption made in many studies of the demand for money function, that $m_{i, t}, y_{i, t}$ and $\Delta \pi_{i, t}$ are of the same order of integration.

Before we start with the analysis we have to remark that we assume, in line with the in section 4.2 introduced models, that there is no contemporaneous correlation between the countries. However, this is not in line with the results found in chapter three where we found contemporaneous correlations for the GDP series. We also expect to find contemporaneous correlations among the other variables as it is very likely that nearby countries will be affected by the same shocks.

There are two reasons for not including the contemporaneous correlations in the models in this first investigation into the use of different sets of restrictions across individuals. First, we wanted to start out in a simple as possible setup, including the contemporaneous correlations would make the analysis more complex. Second, modelling the contemporaneous correlations would generate a relatively large number of additional parameters as we have several variables for each individual which might leave us in practice with very few degrees of freedom. The macroeconomic application considered in this section is viewed as a first practical investigation into the possibilities of using different sets of restrictions across individuals, thereby ignoring the problem of contemporaneous correlation. We admit that for a complete analysis of this system the contemporaneous correlations should be included. This will be done in further research.

The first step in the analysis is selecting the correct order of the VAR. We assume that all countries have the same VAR order. We test for the order of the system in the unrestricted system i.e. we assume to have 6 independent trivariate VAR's of the same order without any further cross-country restrictions. The results for the model selection criteria are given in table 4.1. All three criteria attain their minimun for the system with order two.

On the basis of these results we feel confident that the system can be represented by a system of order two. In the sequel we take a VAR(2) as basis and test for further restrictions in line with the models discussed in this chapter.

We denote the VAR(2) model without any further restrictions by $\Omega$. This model has unrestricted individual lag polynomials and an unrestricted individual symmetric positive definite covariance matrix. As before we denote the other models by the covariance structure notations $\Omega^{p}, \Omega^{i}, \Omega^{s w}$ and $\Omega^{s b}$. 


\begin{tabular}{|l|l|l|}
\hline Test: & $\lambda_{L R}$ & $\mathrm{p}$-value \\
\hline$\Omega^{i}$ versus $\Omega$ & 114.16 & 0.044 \\
\hline$\Omega^{s b}$ versus $\Omega$ & 127.73 & 0.065 \\
\hline$\Omega^{s w}$ versus $\Omega$ & 154.76 & 0.001 \\
\hline$\Omega^{p}$ versus $\Omega$ & 279.79 & $<0.001$ \\
\hline$\Omega^{\text {sb }}$ versus $\Omega^{i}$ & 13.56 & 0.558 \\
\hline$\Omega^{s w}$ versus $\Omega^{i}$ & 40.59 & $<0.001$ \\
\hline$\Omega^{p}$ versus $\Omega^{i}$ & 165.63 & $<0.001$ \\
\hline$\Omega^{p}$ versus $\Omega^{s b}$ & 152.06 & $<0.001$ \\
\hline$\Omega^{p}$ versus $\Omega^{s w}$ & 125.03 & $<0.001$ \\
\hline
\end{tabular}

Table 4.2: Likelihood ratio tests

In Table 4.2 the tests for the different restrictions are given. From the Table it becomes clear that the model denoted by $\Omega^{\text {st }}$ is the most parsimonious one in terms of the number of parameters and is still not rejected by the data. Observe that reject the hypothesis of a common lag structure and unrestricted individual covariance matrix at a $5 \%$ significance level. However for $\Omega^{\text {sh }}$ we do not reject the joint hypothesis of common lag matrices and a common variance structure at a $5 \%$ level. Note also that $\Omega^{s b}$ is clearly to be preferred over the $\Omega^{\text {sw }}$ model.

\subsubsection{Impulse response analysis}

Using the models identified above we perform an impulse response analysis of the system. We calculate the impulse response functions for the unrestricted VAR(2) model and the VAR(2) model with common lag structure and covariance structure $\Omega^{s b}$. We consider the orthogonalized as well as the nonorthogonalized impulse responses. We investigate the effects of the restrictions on the impulse responses and calculate the \pm 2 standard-error bounds.

As the ordering of the variables in Choleski type of decompositions is important for the interpretation of the results, we consider the variables in the following onder $\left(m_{i_{1},}, y_{i_{n}}, p_{i_{i}}\right)^{\prime}$. By choosing a Choleski type of decompostion of the covariance matrices of the disturbances it follows that there is no instantaneous effect of activity and prices on the money balance. It also implies that activity is not instantaneously affected by prices.

We present the results for the Netherlands as a representative country. As directly becomes clear from the graphs the power of the impulse response analysis is increased considerably by the imposed restrictions. The confidence intervals based on the $\Omega^{\text {sb }}$ model, both orthogonalized and nonorthogonalized, are less wide than the confidence intervals based on the data for the Netherlands alone.

We restrict our comment on the impulse responses to the orthogonalized impulse responses. Orthogonalized impulse responses are presented graphically in Figures 4.2 and 4.4. The nonorthogonalized impulse response functions are presented in the Figures 4.1 and 4.3 .

We notice that the first order autocorrelations are all positive and decreasing. This implies a decreasing strength of the effect through time and points to the stationarity of 

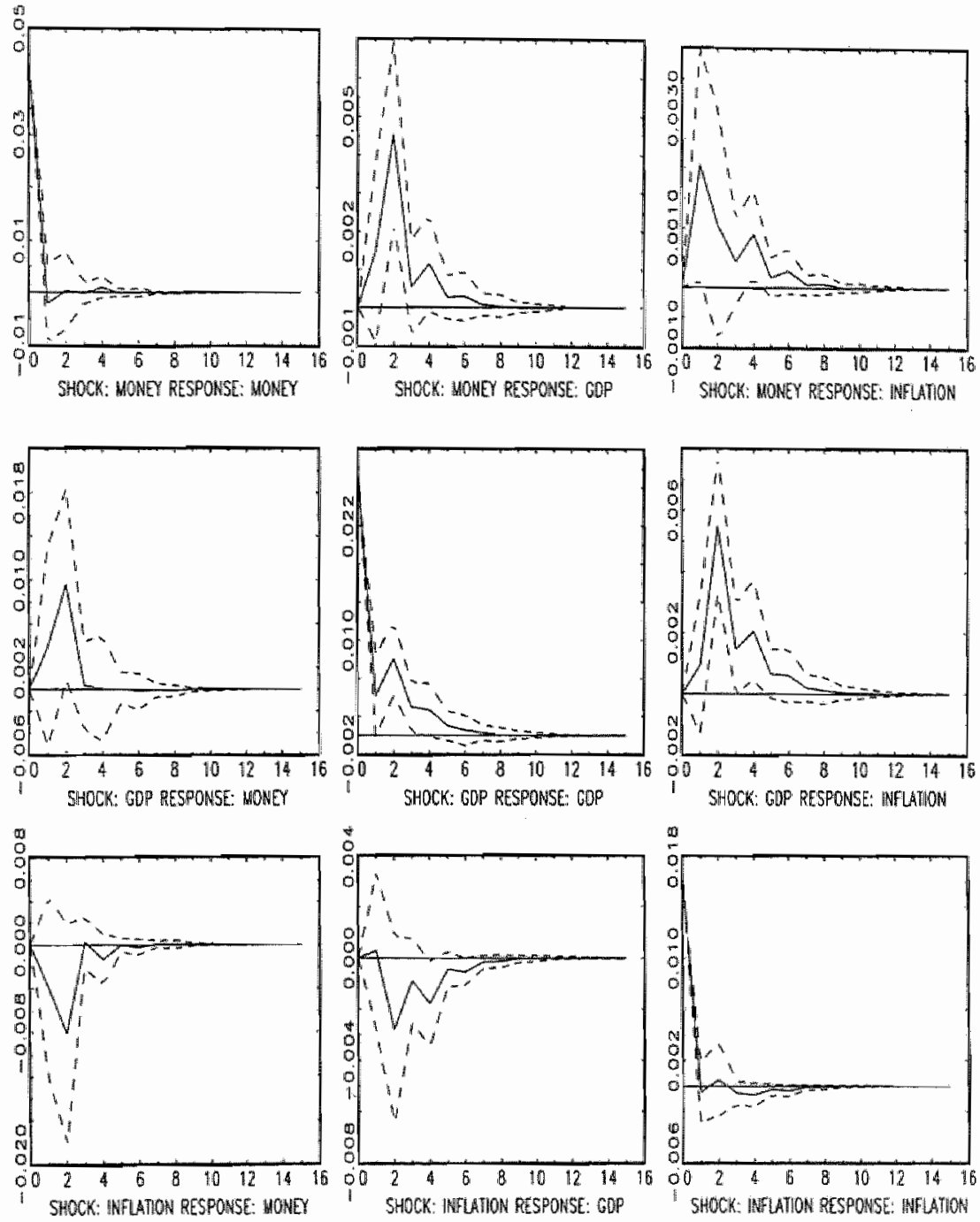

Figure 4.1: Nonorthogonalized impulse responses for the Netherlands based on the $\Omega^{s b}$ mode]. 

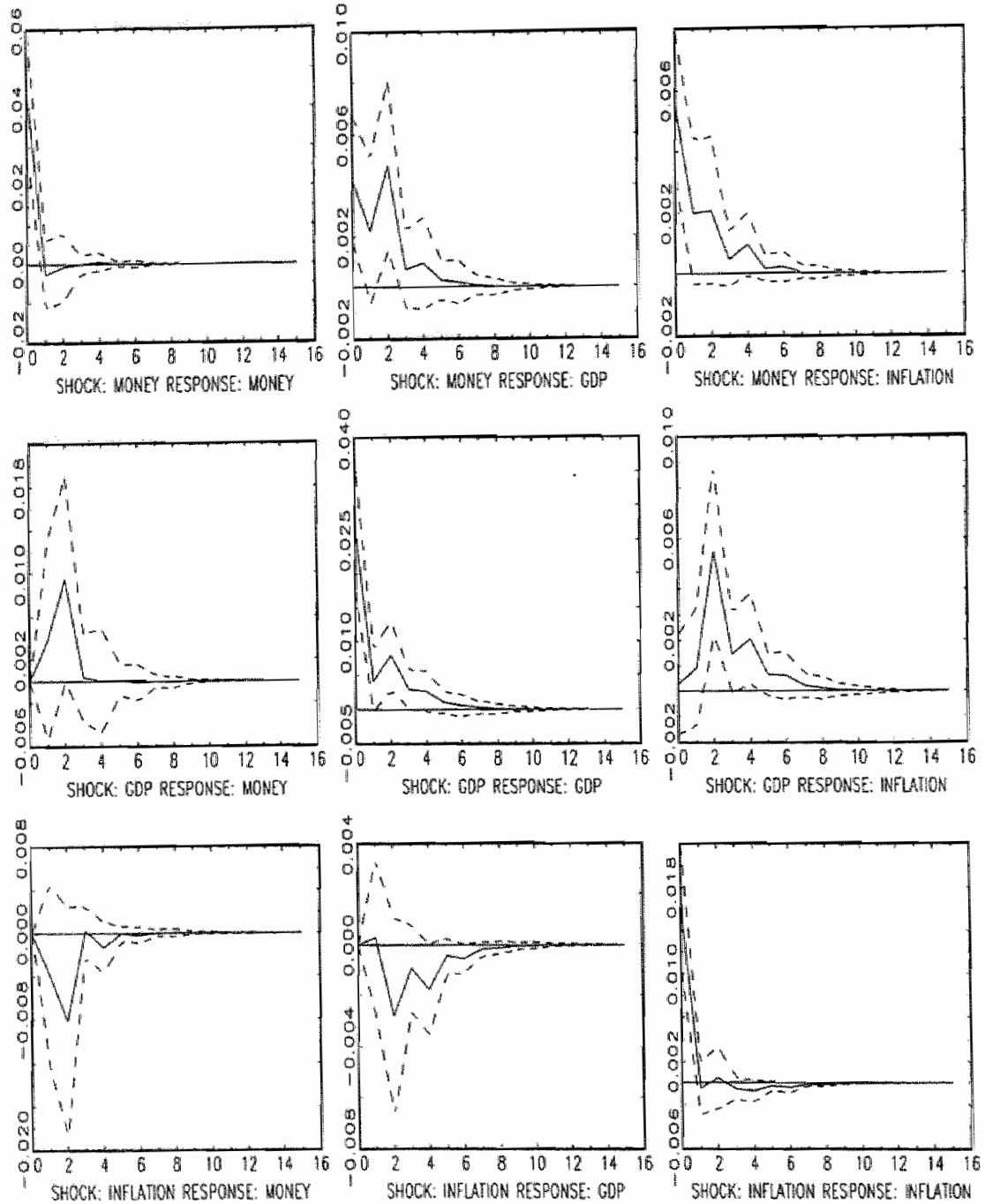

Figure 4.2: Orthogonalized impulse responses for the Netherlands based on the $\Omega^{\text {sb }}$ model. 

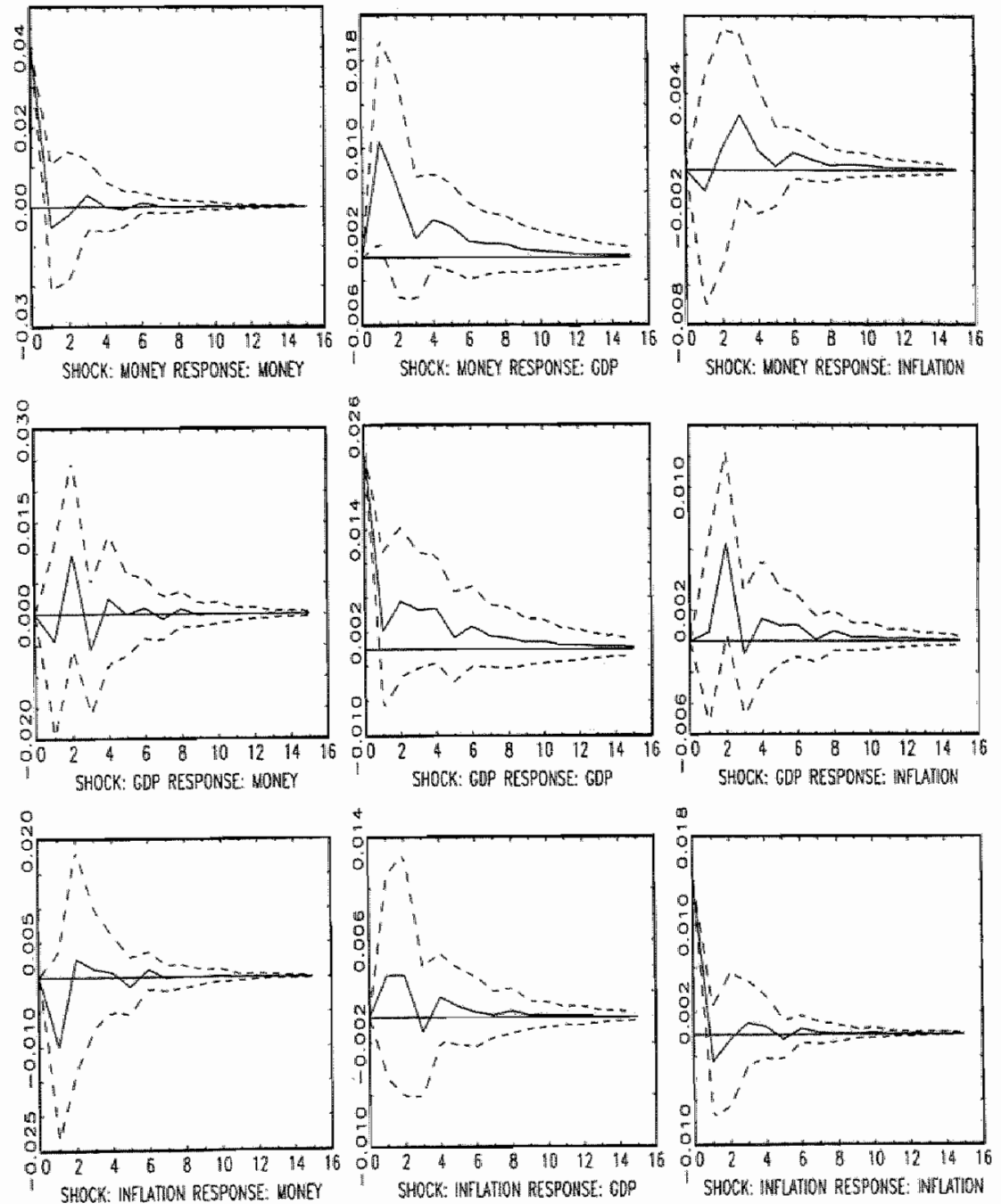

Figure 4.3: Nonorthogonalized impulse responses for the Netherlands based on the individual data. 

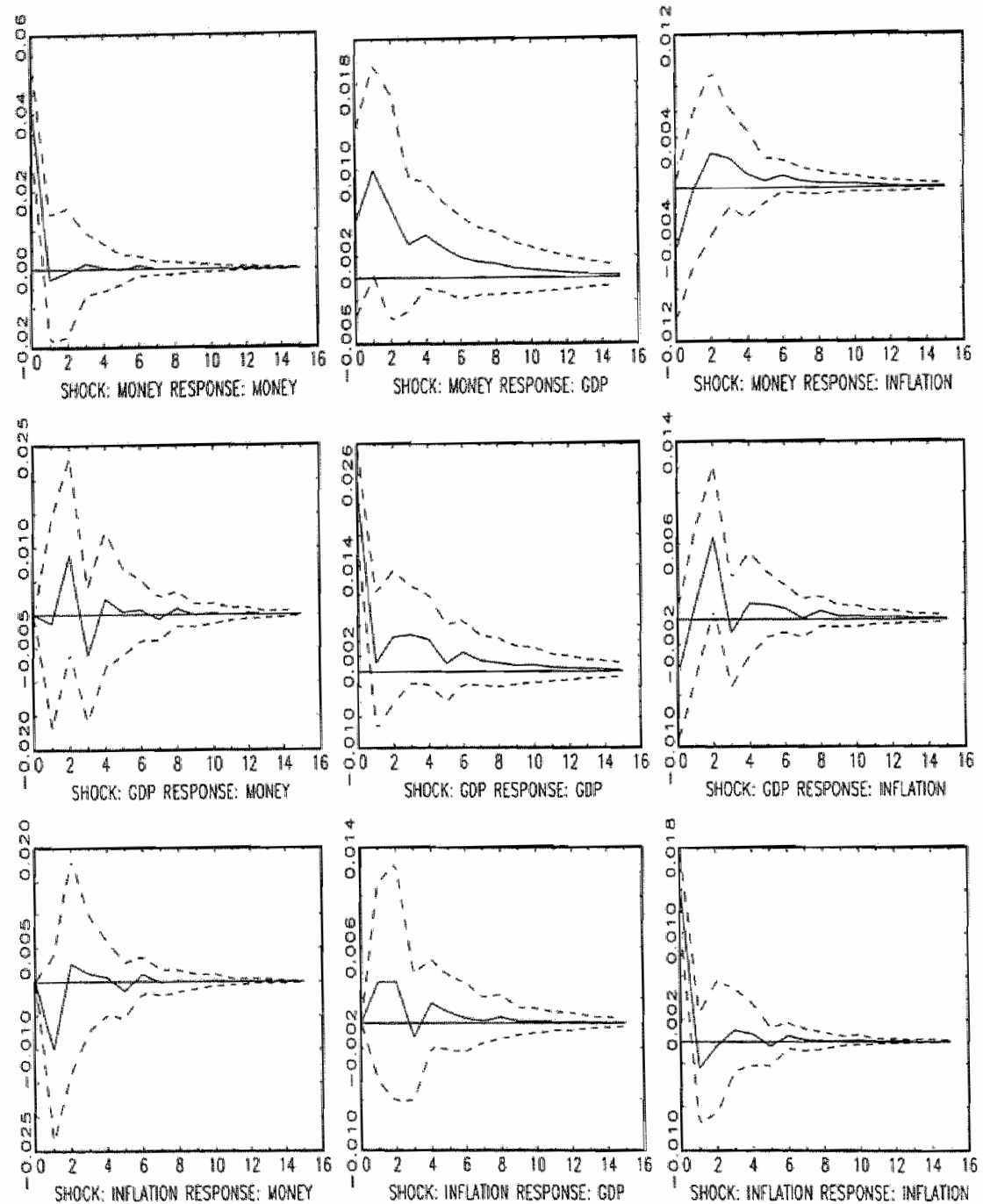

Figure 4.4: Orthogonalized impulse responses for the Netherlands based on the individual data. 
the system. The short-term response of money after a shock in GDP is positive, although not significant. An inflationary shock does not significantly influence the money stock.

As expected, money has a positive impact on activity in the short-term but not in the long-term. Further we find that money has a positive effect in the short term on activity, even if we accept long-term neutrality. This result implies that monetary policy might be an effective instrument to influence activity. The maximum response is reached after two years. This indicates that it takes a some time for a shock in money to get transmitted to the real economy.

The impact of inflation on GDP is not significant. This result may be specific for our sample which consists of countries which, during the observed period, have been targeting low inflation whatever the fluctuations of activity.

The reaction of inflation after a (real) monetary shock is positive. After a positive monetary shock there is an increase in demand which leads to a rise in inflation. Finally, a natural interpretation of the impact of a shock of GDP on inflation would be a demand shock.

We point out that most of these conclusions cannot be drawn from the impulse response analysis based on the data of the individual countries alone. As we have jointly estimated the model for 6 countries we could also have presented here the results for any of the other 5 countries. We note that in the $\Omega^{s b}$ model the impulse responses for the same variable for the different individuals only differs with a multiplicative constant corresponding to the ratio of the standard deviations of the variable of the shock for the different individuals. The confidence bounds are also different for each individual as can be seen from Theorem 19.

\subsection{Conclusions}

In this chapter we show that efficiency gains in the estimation of multiple vector autoregressions can be obtained using cross-country restrictions. We impose a common lag polynomial structure but we leave the intercepts for the different countries unrestricted. A second set of restrictions is introduced in the structure of the covariance matrix of each individual country. We propose parametrizations which decompose the covariance matrix into a product of matrices, the parameters of which are either common to all countries or country-specific.

Using the asymptotic distributions of the MLE and LSE we show the usefulness of this type of models by demonstrating the additional power of the impulse response analysis resulting from imposing cross-country restrictions.

Further extensions will include the analysis of the models with these sets of restric tions to nonstationary, possibly cointegrated vector autoregressions. Another interesting extension is to change the Choleski type of decomposition of the covariance structure to identify shocks to structures like the Blanchard and Quah (1989) type. Another extension should also include deriving results for Forecast Error Variance Decomposition. 


\subsection{Appendix A: Technical results}

In this appendix we prove the results which were only stated in the main text. We start by introducing some additional notation and results.

\subsubsection{Introduction and notation}

A number of special matrices are used in the derivations. These special matrices are important when studying the class of so-called L-structured matrices. We refer to Magnus (1988), Magnus and Neudecker (1986) and (1991) and Lutkepoh! (1996) for further references and more results.

L-structured matrices are real matrices of a certain dimension and order that satisfy a given set of linear restrictions. We introduce the matrices $L_{k}, D_{k}, K_{n, m}, \tilde{L}_{k}$ and $\Psi_{k}^{\prime}$ with the following properties:

1. The Elimination matrix $L_{k}$ is defined as the $\left(\frac{1}{2} k(k+1) \times k^{2}\right)$ matrix such that

$$
\operatorname{vec}(A)=L_{k}^{\prime} \operatorname{vech}(A)
$$

for every lower triangular $(k \times k)$ matrix $A$;

2. The Duplication matrix $D_{k}$ is defined as the $\left(k^{2} \times \frac{1}{2} k(k+1)\right)$ matrix with the property that

$$
\operatorname{vec}(A)=D_{k} \operatorname{vech}(A)
$$

for every symmetric $(k \times k)$ matrix $A$;

3. The matrix $\Psi_{k}$ is the $\left(k \times k^{2}\right)$ matrix with the property that

$$
\operatorname{vec}(A)=\Psi_{k}^{\prime} w(A)
$$

for every diagonal $(k \times k)$ matrix $A$. Here the operator $w(\bullet)$ is defined as $w(A)=$ $\left(a_{1,1}, a_{2,2, \ldots,}, a_{k, k}\right)^{\prime}$.

4. The Commutation matrix $K_{n, m}$ is defined by the property that for any $(n \times m)$ matrix $A$ the relation

holds.

$$
K_{n, m} \operatorname{vec}(A)=\operatorname{vec}\left(A^{\prime}\right)
$$

5. The $\left(\frac{1}{2} k(k-1) \times k^{2}\right)$ matrix $\breve{L}_{k}$ is the matrix with the property that

$$
\operatorname{vec}(A)=\tilde{L}_{k}^{\prime} v(A)
$$

for every strictly lower triangular matrix $A$. Here the operator $v(\bullet)$ is defined as taking out all the elements below the main diagonal i.e. $v(A)=\left(a_{2,1}, \ldots, a_{2, k}, a_{3,2}, \ldots, a_{3, k}, \ldots, a_{k, k}\right)^{\prime}$.

Finally we introduce the matrix $N_{k}=\frac{1}{2}\left(I_{k^{2}}+K_{k, k}\right)$ which plays an important role in many calculations involving the above defined matrices, such as derivatives of functions of structured matrices. 


\subsubsection{Proofs of Theorems 14 and 15}

In this section we prove the theorems 14 and 15 .

Proof. (proof of theorem 14.) The likelihood function given in (4.30) can be restated as

$$
\begin{aligned}
L & \propto \frac{T}{2} \sum_{i=1}^{N} \log \left(\left|\Omega_{i}\right|^{-1}\right)-\frac{1}{2} \sum_{i=1}^{N} \sum_{t=1}^{T} \operatorname{trace}\left(\left(Y_{i, t}-\Phi X_{i, t}\right)^{\prime} \Omega_{i}^{-1}\left(Y_{i, t}-\Phi X_{i, t}\right)\right) \\
& =\frac{1}{2} \sum_{i=1}^{N} \sum_{t=1}^{T}\left(\log \left(\left|\Omega_{i}\right|^{-1}\right)-\operatorname{trace}\left(\left(Y_{i, t}-\Phi X_{i, t}\right)^{\prime} \Omega_{i}^{-1}\left(Y_{i, t}-\Phi X_{i, t}\right)\right)\right) \\
& =\frac{1}{2} \sum_{i=1}^{N} \sum_{t=1}^{T}\left(\log \left(\left|\Omega_{i}\right|^{-1}\right)-\operatorname{trace}\left(u_{i, t}^{t} \Omega_{i}^{-1} u_{i, t}\right)\right) .
\end{aligned}
$$

where $u_{i, t}=\left(Y_{i, t}-\Phi X_{i, t}\right)$. The first order derivative for the parameter vector $\theta$, the argument of the covariance matrix, is given by

$$
\begin{aligned}
\frac{\partial L}{\partial \theta^{\prime}} & =\frac{1}{2} \sum_{i=1}^{N} \sum_{t=1}^{T} \frac{\partial}{\partial \theta^{\prime}}\left(\log \left(\left|\Omega_{i}\right|^{-1}\right)-\operatorname{trace}\left(u_{i, t}^{\prime} \Omega_{i}^{-1} u_{i, t}\right)\right) \\
& =\frac{1}{2} \sum_{i=1}^{N} \sum_{t=1}^{T} \frac{\partial^{\prime}}{\partial \operatorname{vec}\left(\Omega_{i}\right)^{\prime}}\left(-\log \left(\left|\Omega_{i}\right|\right)-\operatorname{trace}\left(u_{i, t}^{\prime} \Omega_{i}^{-1} u_{i, t}\right)\right) \frac{\partial \operatorname{vec}\left(\Omega_{i}\right)}{\partial \theta^{\prime}} \\
& =-\frac{1}{2} \sum_{i=1}^{N} \sum_{t=1}^{T}\left(\operatorname{vec}\left(\Omega_{i}^{-1}-\Omega_{i}^{-1} u_{i, t} u_{i, t}^{\prime} \Omega_{i}^{-1}\right)\right)^{\prime} \frac{\partial \operatorname{vec}\left(\Omega_{i}\right)}{\partial \theta^{\prime}}
\end{aligned}
$$

For the parameters of the $\Phi$ of the lag polynomial we have

$$
\begin{aligned}
\frac{\partial L}{\partial \Phi}= & -\frac{1}{2} \sum_{i=1}^{N} \sum_{t=1}^{T} \frac{\partial}{\partial \Phi} \operatorname{trace}\left(\Omega_{i}^{-1}\left(Y_{i, t}-\Phi X_{i, t}\right)\left(Y_{i, t}-\Phi X_{i, t}\right)^{\prime}\right) \\
= & -\frac{1}{2} \sum_{i=1}^{N} \sum_{t=1}^{T} \frac{\partial}{\partial \Phi} \operatorname{trace}\left(\Omega_{i}^{-1} Y_{i, t} Y_{i, t}^{\prime}-\Omega_{i}^{-1} Y_{i, t} X_{i, t}^{\prime} \Phi^{\prime}\right. \\
& \left.-\Omega_{i}^{-1} \Phi X_{i, t} Y_{i, t}^{\prime}+\Omega_{i}^{-1} \Phi X_{i, t} X_{i, t^{\prime}}^{\prime} \Phi^{\prime}\right) \\
= & -\frac{1}{2} \sum_{i=1}^{N} \sum_{t=1}^{T}\left(-2 \Omega_{i}^{-1} Y_{i, t} X_{i, t}^{\prime}+2 \Omega_{i}^{-1} \Phi X_{i, t} X_{i, t}^{\prime}\right) \\
= & \sum_{i=1}^{N} \sum_{t=1}^{T}\left(\Omega_{i}^{-1}\left(Y_{i, t} X_{i, t}^{\prime \prime}-\Phi X_{i, t} X_{i, t}^{i}\right)\right) .
\end{aligned}
$$

Putting this first order derivative equal to zero gives

$$
\sum_{i=1}^{N} \sum_{t=1}^{T}\left(x_{i, t} \otimes \Omega_{i}^{-1}\right) Y_{i, t}=\sum_{i=1}^{N} \sum_{t=1}^{T}\left(x_{i, t} x_{i, t}^{\prime} \otimes \Omega_{i}^{-1}\right) \operatorname{vec}(\Phi)
$$


or

$$
\alpha=\left[\sum_{i=1}^{N} \sum_{t=1}^{T}\left(X_{i, t} X_{i, t}^{\prime} \otimes \Omega_{i}^{-1}\right)\right]^{-1} \sum_{i=1}^{N} \sum_{t=1}^{T}\left(X_{i, t} \otimes \Omega_{i}^{-1}\right) Y_{i, t}
$$

where $a=v e c(\Phi)$. For the fixed effects, or individual means, we have

$$
\begin{aligned}
\frac{\partial L}{\partial \mu_{i}} & =-\frac{\partial}{\partial \mu_{i}}\left(\frac{1}{2} \sum_{i=1}^{N} \sum_{t=1}^{T} \operatorname{trace}\left(u_{i, t}^{t} \Omega_{i}^{-1} u_{i, t}\right)\right) \\
& =-\sum_{t=1}^{T} \frac{\partial u_{i, t}^{\prime}}{\partial \mu_{i}} \Omega_{i}^{-1} u_{i, t} \\
& =-\sum_{t=1}^{T} \frac{\partial\left(Y_{i, t}-\Phi X_{i, t}\right)^{\prime}}{\partial \mu_{i}} \Omega_{i}^{-1}\left(Y_{i, t}-\Phi X_{i, t}\right)
\end{aligned}
$$

Observe now that

$$
\begin{aligned}
Y_{i, t}-\Phi X_{i, t} & =\left(y_{i, t}-\mu_{i i}\right)-\Phi_{1}\left(y_{i, t-1}-\mu_{i}\right)-\ldots-\Phi_{p}\left(y_{i_{k} t-p}-\mu_{i}\right) \\
& =y_{i, t}-\Phi x_{i, t}-\left(I-\Phi_{1}-. .-\Phi_{p}\right) \mu_{i}
\end{aligned}
$$

which gives

$$
\frac{\partial\left(Y_{i, t}-\Phi X_{i, t}\right)^{\prime}}{\partial \mu_{i}}=-I+\Phi_{1}^{\prime}+\ldots+\Phi_{p}^{\prime}
$$

This leads to

$$
\frac{\partial L}{\partial \mu_{i}}=\left(I-\Phi_{1}-\ldots-\Phi_{p}\right)^{\prime} \Omega_{i}^{-1} \sum_{t=1}^{T}\left(Y_{i, t}-\Phi X_{i, t}\right)
$$

Now solving for $\mu_{i}$ gives

$$
\mu_{i}=\frac{1}{T}\left[\left(I-\Phi_{1}-\ldots-\Phi_{p}\right)\right]^{-1} \sum_{t=1}^{T}\left(y_{i, t}-\Phi x_{i, t}\right)
$$

Proof. (Proof of Theorem 15) As the covariance matrix of the ML estimator is pro portional to the Hessian of the likelihood function we first calculate the Hessian of the likelihood function. The second order derivatives for the individual means are given by

$$
\frac{\partial^{2} L}{\partial \mu_{j} \partial \mu_{i}^{l}}=\left\{\begin{array}{lll}
0, & \text { if } & i \neq j \\
-T\left(I-\Phi_{1}-\ldots-\Phi_{p}\right)^{\prime} \Omega_{i}^{-1}\left(I-\Phi_{1}-\ldots-\Phi_{p}\right), & \text { if } & i=j
\end{array}\right.
$$

The second order derivatives of the individual effects with the other parameters are given by

$$
\frac{\partial^{2} L}{\partial \mu_{i} \partial \theta^{p}}=-\frac{\partial}{\partial \theta^{t}}\left(\sum_{t=1}^{T} \frac{\partial u_{i, t}^{t}}{\partial \mu_{i}} \Omega_{i}^{-1} u_{i, t}\right)
$$




$$
\begin{aligned}
& =-\frac{\partial}{\partial \theta^{t}}\left(\sum_{t=1}^{T} \operatorname{vec}\left(\frac{\partial u_{i, t}^{t}}{\partial \mu_{i}} \Omega_{i}^{-1} u_{i, t}\right)\right) \\
& =-\left(\sum_{t=1}^{T} \frac{\partial \operatorname{vec}\left(\frac{\partial u_{i, t}^{\prime}}{\partial \mu_{i}} \Omega_{i}^{-1} u_{i, t}\right)}{\partial \operatorname{vec}\left(\Omega_{i}\right)^{\prime}} \frac{\partial \operatorname{vec}\left(\Omega_{i}\right)}{\partial \theta^{t}}\right) \\
& =-\sum_{t=1}^{T}\left(u_{i, r_{i}^{\prime}}^{t} \Omega_{i}^{-1} \otimes \frac{\partial u_{i, t}^{\prime}}{\partial \mu_{i}} \Omega_{i}^{-1}\right) \frac{\partial \operatorname{vec}\left(\Omega_{i}\right)}{\partial \theta^{\prime}} .
\end{aligned}
$$

And further

$$
\begin{aligned}
\frac{\partial^{2} L}{\partial \mu_{i} \partial \operatorname{vec}(\Phi)^{\prime}} & =\frac{\partial}{\partial \mu_{i}} \operatorname{vec}\left(\sum_{i=1}^{N} \sum_{t=1}^{T}\left(\Omega_{i}^{-1} u_{i, t} X_{i, t}^{\prime}\right)\right)^{\prime} \\
& =\sum_{t=1}^{T} \frac{\partial}{\partial \mu_{i}} \operatorname{vec}\left(\Omega_{i}^{-1} u_{i, t} X_{i, t}^{\prime}\right)^{\prime}=\sum_{t=1}^{T}\left(\frac{\partial \operatorname{vec}\left(\Omega_{i}^{-1} u_{i, t} I X_{i, t} I\right)}{\partial \mu_{i}^{\prime}}\right)^{\prime} \\
& =\sum_{t=1}^{T}\left(\left(I \otimes \Omega_{i}^{-1}{\left.u_{i, t} I\right)}^{\prime} \frac{\partial \operatorname{vec}\left(u_{i, t}\right)}{\partial \mu_{i}}+\left(X_{i, t} \otimes \Omega_{i}^{-1}\right) \frac{\partial \operatorname{vec}\left(X_{i, t}^{\prime}\right)}{\partial \mu_{i}}\right)\right.
\end{aligned}
$$

Now observe that both last expressions have expectation zero.

$$
\begin{aligned}
\frac{\partial^{2} L}{\partial \theta \partial \operatorname{vec}(\Phi)^{\prime}} & =\frac{\partial}{\partial \theta} \operatorname{vec}\left(\sum_{i=1}^{N} \sum_{t=1}^{T}\left(\Omega_{i}^{-1} u_{i, t} X_{i, t}^{\prime}\right)\right)^{\prime} \\
& =\sum_{i=1}^{N} \sum_{t=1}^{T} \frac{\partial}{\partial \theta} \operatorname{vec}\left(\Omega_{i}^{-1} u_{i, t} X_{i, t}^{\prime}\right)^{\prime} \\
& =\sum_{i=1}^{N} \sum_{t=1}^{T} \frac{\partial \operatorname{vec}\left(\Omega_{i}^{-1}\right)^{\prime}}{\partial \theta} \frac{\partial \operatorname{vec}\left(\Omega_{i}^{-1} u_{i, t} X_{i, t}^{\prime}\right)^{\prime}}{\partial \operatorname{vec}\left(\Omega_{i}^{-1}\right)} \\
& =\sum_{i=1}^{N} \sum_{t=1}^{T} \frac{\partial \operatorname{vec}\left(\Omega_{i}^{-1}\right)^{\prime}}{\partial \theta}\left(X_{i, t} u_{i, t}^{\prime} \otimes I_{i, k}\right)^{\prime}
\end{aligned}
$$

Taking expectations one can immediately see that this equals zero by the observation that $E\left(X_{i, t} u_{i, t}^{i}\right)=0$. We further have

$$
\begin{aligned}
\frac{\partial^{2} L}{\partial \operatorname{vec}(\Phi) \partial \operatorname{vec}(\Phi)^{\prime}}= & -\frac{\partial L}{\partial \operatorname{vec}(\Phi)} \frac{\partial L}{\partial \operatorname{vec}(\Phi)^{\prime}} \\
= & -\frac{\partial L}{\partial \operatorname{vec}(\Phi)}\left(\frac{\partial L}{\partial \operatorname{vec}(\Phi)}\right)^{\prime} \\
= & -\operatorname{vec}\left(\sum_{i=1}^{N} \sum_{t=1}^{T}\left(\Omega_{i}^{-1} u_{i, t} X_{i, t}\right)\right) \\
& \quad \times \operatorname{vec}\left(\sum_{i=1}^{N} \sum_{t=1}^{T}\left(\Omega_{i}^{-1} u_{i, t} X_{i, t}\right)\right)^{\prime}
\end{aligned}
$$




$$
\begin{aligned}
& =-\sum_{i=1}^{N} \sum_{t=1}^{T} \sum_{j=1}^{N} \sum_{s=1}^{T} \operatorname{vec}\left(\Omega_{i}^{-1} u_{i, t} X_{i, t}\right) \operatorname{vec}\left(\Omega_{j}^{-1} u_{j, s} x_{j, s}^{\prime}\right)^{\prime} \\
& =-\sum_{i=1}^{N} \sum_{t=1}^{T} \sum_{j=1}^{N} \sum_{s=1}^{T}\left(X_{i, t} \otimes \Omega_{i}^{-1}\right) u_{i, t} u_{j, s}^{\prime}\left(X_{i, t} \otimes \Omega_{i}^{-1}\right) .
\end{aligned}
$$

Taking expectations and observing that $E\left(X_{i, t} X_{i, t}^{t}\right)=\Gamma^{i}(p)$ we have

$$
\begin{aligned}
-E\left(\frac{1}{N T} \frac{\partial^{2} L}{\partial \operatorname{vec}(\Phi) \operatorname{\partial vec}(\Phi)^{\prime}}\right) & =E \sum_{i=1}^{N}\left(X_{i, t} \otimes \Omega_{i}^{-1}\right) u_{i, t} u_{i, s}^{\prime}\left(X_{i, t}^{\prime} \otimes \Omega_{i}^{-1}\right) \\
& =E \sum_{i=1}^{N}\left(X_{i, t} \otimes \Omega_{i}^{-1}\right) \Omega_{i}\left(X_{i, t}^{\prime} \otimes \Omega_{i}^{-1}\right) \\
& =E \sum_{i=1}^{N}\left(X_{i, t} X_{i, t}^{\prime} \otimes \Omega_{i}^{-1}\right) \\
& =\frac{1}{N} \sum_{i=1}^{N}\left(\Gamma^{i}(p) \otimes \Omega_{i}^{-1}\right) .
\end{aligned}
$$

Finally we obtain the second order derivatives with respect to the parameters of the covariance matrix

$$
\begin{aligned}
\frac{\partial^{2} L}{\partial \theta \partial \theta^{\prime}} & =\frac{1}{2} \sum_{i=1}^{N} \sum_{t=1}^{T} \frac{\partial^{2}\left(\log \left(\left|\Omega_{i}\right|^{-1}\right)-\operatorname{trace}\left(u_{i, t}^{\prime} \Omega_{i}^{-1} u_{i, t}\right)\right)}{\partial \theta \partial \theta^{\prime}} \\
& =\frac{1}{2} \sum_{i=1}^{N} \sum_{t=1}^{T} \frac{\partial \operatorname{vec}\left(\Omega_{i}\right)^{r}}{\partial \theta} \frac{\partial^{2}\left(\log \left(\left|\Omega_{i}\right|^{-1}\right)-\operatorname{trace}\left(u_{i, t}^{\prime} \Omega_{i}^{-1} u_{i, t}\right)\right)}{\partial \operatorname{vec}\left(\Omega_{i}\right) \partial \operatorname{vec}\left(\Omega_{i}\right)^{\prime}} \frac{\partial \operatorname{vec}\left(\Omega_{i}\right)}{\partial \theta^{*}} \\
& =\frac{1}{2} \sum_{i=1}^{N} \sum_{i=1}^{T} \frac{\partial \operatorname{vec}\left(\Omega_{i}\right)^{\prime}}{\partial \theta} M_{i} \frac{\partial \operatorname{vec}\left(\Omega_{i}\right)}{\partial \theta^{\prime}}
\end{aligned}
$$

with

$$
M_{i}=K_{k, k}\left[\Omega_{i}^{-1} \otimes \Omega_{i}^{-1}-\Omega_{i}^{-1} u_{i, t} u_{i, t}^{\prime} \Omega_{i}^{-1} \otimes \Omega_{i}^{-1}-\Omega_{i}^{-1} \otimes \Omega_{i}^{-1} u_{i, t} u_{i, t}^{\prime} \Omega_{i}^{-1}\right],
$$

where we used

$$
\frac{\partial^{2}\left(\log \left(\left|\Omega_{i}\right|^{-1}\right)\right)}{\partial \operatorname{vec}\left(\Omega_{i}\right) \partial \operatorname{vec}\left(\Omega_{i}\right)^{\prime}}=-K_{k, k}\left[\Omega_{i}^{-1} \otimes \Omega_{i}^{-1}\right]
$$

and

$$
\frac{\partial^{2}\left(\operatorname{trace}\left(u_{i, t}^{i} \Omega_{i}^{-1} u_{i, t}\right)\right)}{\partial \operatorname{vec}\left(\Omega_{i}\right) \partial \operatorname{vec}\left(\Omega_{i}\right)^{i}}=K_{k, k}\left[\Omega_{i}^{-1} u_{i, t} u_{i, t}^{i} \Omega_{i}^{-1} \otimes \Omega_{i}^{-1}+\Omega_{i}^{-1} \otimes \Omega_{i}^{-1} u_{i, t} u_{i, t}^{\prime} \Omega_{i}^{-1}\right] .
$$


Taking expectations leads to

$$
\begin{aligned}
-E\left(\frac{1}{N T} \frac{\partial^{2} L}{\partial \theta \partial \theta^{\prime}}\right) & =\frac{1}{2 N T} \sum_{i=1}^{N} \sum_{t=1}^{T} \frac{\partial v e c\left(\Omega_{i}\right)^{\prime}}{\partial \theta} E\left(M_{i}\right) \frac{\partial v e c\left(\Omega_{i}\right)}{\partial \theta^{\prime}} \\
& =\frac{1}{2 N} \sum_{i=1}^{N} \frac{\partial v e c\left(\Omega_{i}\right)^{\prime}}{\partial \theta} K_{k, k}\left[\Omega_{i}^{-1} \otimes \Omega_{i}^{-1}\right] \frac{\partial v e c\left(\Omega_{i}\right)}{\partial \theta^{\prime}}
\end{aligned}
$$

Gathering all the above results to form the information matrix and inverting it gives the stated result.

\subsubsection{Proofs of Theorems 16 and 17}

From Theorem 14 it follows that $\frac{\partial v e c\left(\Omega_{i}\right)}{\partial \theta}$ determines the behavior of the covariance estimators in the different models. We therefore state the following lemma. The abbreviation (L:186,1) indicates a reference to Luitkepohl (1996) page 186, formula 1.

Lemma 20 The following relations hold

$$
\begin{aligned}
& \frac{\partial v e c\left(\Omega_{i}\right)}{\partial \theta^{p t}}=D_{k} \\
& \frac{\partial v e c\left(\Omega_{i}\right)}{\partial \theta^{i j}}=\left(e_{i, N}^{\prime} \otimes D_{k}\right) \\
& \frac{\partial v e c\left(\Omega_{i}\right)}{\partial \theta^{s b}}=2 N_{k}\left[\left(\Gamma \Sigma_{i}^{2} \otimes I_{k}\right) \tilde{L}_{k}^{\prime}:\left(e_{i, N}^{\prime} \otimes\left(\Gamma \otimes \Gamma \Sigma_{i}\right) \Psi_{k}^{\prime}\right)\right] \\
& \frac{\partial v e c\left(\Omega_{i}\right)}{\partial \theta^{s w r}}=2 N_{k}\left[\left(\Sigma_{i} \Gamma \otimes \Sigma_{i}\right) \tilde{L}_{k}^{\prime}:\left(e_{i, N}^{\prime} \otimes\left(\Sigma_{i} \Gamma \Gamma^{\prime} \otimes I_{k}\right) \Psi_{k}^{\prime}\right)\right]
\end{aligned}
$$

where the $(N \times 1)$ vector of zenas with a one on the $i$ - th place is denoted by $e_{i, N}^{\prime}=$ $(0, \ldots, 1, \ldots, 0)^{r}$.

Proof. The result (4.50) follows immediately from

$$
\frac{\partial v e c\left(\Omega_{i}\right)}{\partial \theta^{p \prime}}=\frac{\partial v e c(\Omega)}{\partial v e c h(\Omega)^{\prime}}=D_{k}
$$

noting that $\Omega$ is symmetric (see $L: 186,1$ ). The result for (4.51) follows also directly from

$$
\begin{aligned}
\frac{\partial \operatorname{vec}\left(\Omega_{i}\right)}{\partial \theta^{i t}} & =\frac{\partial v e c\left(\Omega_{i}\right)}{\left[\partial \operatorname{vech}\left(\Omega_{1}\right)^{r}, \ldots, \partial \operatorname{vech}\left(\Omega_{N}\right)^{\prime}\right]} \\
& =\left[O_{k}, \ldots, O_{k}, D_{k}, O_{k}, . ., O_{k}\right] \\
& =\left(e_{i, N}^{\prime} \otimes D_{k}\right) .
\end{aligned}
$$

For the other two derivatives we have to do a little more work. Using the chain rule and

$$
\frac{\partial v e c(\Gamma)}{\partial v(\Gamma)^{\prime}}=\tilde{L}_{k}^{\prime}, \quad \frac{\partial v e c\left(\Sigma_{i}\right)}{\partial w\left(\Sigma_{i}\right)^{\prime}}=\Psi_{k}^{\prime}
$$


which follows from the fact that $\Gamma$ is lower triangular and $\Sigma_{i}$ is diagonal, we obtain for the parameters of the covariance structure $\Omega^{s t}$

$$
\begin{aligned}
& \frac{\partial v e c\left(\Omega_{i}\right)}{\partial \theta^{s b r}}=\frac{\partial v e c\left(\Gamma \Sigma_{i}^{2} \Gamma^{\prime}\right)}{\left[\partial v(\Gamma)^{\prime}, \partial w\left(\Sigma_{1}\right)^{\prime}, \ldots, \partial w\left(\Sigma_{N}\right)^{\prime}\right]} \\
& =\left[\frac{\partial \operatorname{vec}\left(\Gamma \Sigma_{i}^{2} \Gamma^{\prime}\right)}{\partial \operatorname{vec}(\Gamma)^{\prime}} \widetilde{L}_{k}^{\prime}: \frac{\partial \operatorname{vec}\left(\Gamma \Sigma_{i}^{2} \Gamma^{\prime}\right)}{\partial \operatorname{vec}\left(\Sigma_{1}\right)^{\prime}} \Psi_{k}^{\prime}: \ldots: \frac{\partial \operatorname{vec}\left(\Gamma \Sigma_{i}^{2} \Gamma^{\prime}\right)}{\partial \operatorname{vec}\left(\Sigma_{N}\right)^{\prime}} \Psi_{k}^{\prime}\right] \\
& =\left[\left(I_{k^{2}}+K_{k k}\right)\left(\Gamma \Sigma_{i}^{2} \otimes I_{k}\right) \tilde{L}_{k}^{\prime \prime}:\left(e_{i, N}^{\prime} \otimes\left(I_{k^{2}}+K_{k k}\right)\left(\Gamma \otimes \Gamma \Sigma_{i}\right) \Psi_{k}^{\prime}\right)\right] \\
& =2\left[\left(\Gamma \Sigma_{i}^{2} \otimes I_{k}\right)\left(\Gamma \Sigma_{i}^{2} \otimes I_{k}\right) \tilde{L}_{k}^{\prime}:\left(e_{i, N}^{\prime} \otimes\left(\Gamma \otimes \Gamma \Sigma_{i}\right) \Psi_{k}^{\prime}\right)\right] .
\end{aligned}
$$

The third equality follows from (L:190,7) and (L:190,10). In a similar way we obtain

$$
\begin{aligned}
& \frac{\partial v e c\left(\Omega_{i}\right)}{\partial \theta^{s w \prime}}=\frac{\partial v e c\left(\Sigma_{i} \Gamma \Gamma^{\prime} \Sigma_{i}\right)}{\left[\partial \operatorname{vec}(\Gamma)^{\prime}, \partial w\left(\Sigma_{1}\right)^{\prime}, \ldots, \partial w\left(\Sigma_{N}\right)^{\prime}\right]} \\
& =\left[\frac{\partial v e c\left(\Sigma_{i} \Gamma \Gamma^{\prime} \Sigma_{i}\right)}{\partial v e c(\Gamma)^{\prime}} \stackrel{\sim}{L}_{k}^{\prime}: \frac{\partial v e c\left(\Sigma_{i} \Gamma \Gamma^{\prime} \Sigma_{i}\right)}{\partial w\left(\Sigma_{1}\right)^{\prime}} \Psi_{k}^{\prime}: \ldots: \frac{\partial v e c\left(\Sigma_{i} \Gamma \Gamma^{\prime} \Sigma_{i}\right)}{\partial w\left(\Sigma_{N}\right)^{\prime}} \Psi_{k}^{\prime}\right] \\
& =\left[\left(I_{k^{2}}+K_{k k}\right)\left(\Sigma_{i} \Gamma \otimes \Sigma_{i}\right) \breve{L}_{k}^{\prime}\right. \text { : } \\
& \left.\left(e_{i, N}^{\prime} \otimes\left(I_{k^{2}}+K_{k k}\right)\left(\Sigma_{i} \Gamma \Gamma^{\prime} \otimes I_{k}\right) \Psi_{k}^{\prime}\right)\right] \\
& =2 N_{k}\left[\left(\Sigma_{i} \Gamma \otimes \Sigma_{i}\right) \tilde{L}_{k}^{\prime}:\left(e_{i, N}^{i} \otimes\left(\Sigma_{i} \Gamma \Gamma^{i} \otimes I_{k}\right) \Psi_{k}^{\prime}\right)\right],
\end{aligned}
$$

where we used (L:190,7).and (L:190,12).

Proof. (Proof of Theorem 16) We recall that the first order condition in terms of $\theta$ is given by

$$
0=\frac{1}{2} \sum_{i=1}^{N} \sum_{i=1}^{T}\left(\operatorname{vec}\left(\Omega_{i}^{-1}-\Omega_{i}^{-1} u_{i, t} u_{i, t}^{r} \Omega_{i}^{-1}\right)\right)^{\prime} \frac{\partial v e c\left(\Omega_{i}\right)}{\partial \theta^{\prime}} .
$$

Using Lemma 20 we have for the model with the pooled covariance structure $\Omega^{p}, \Omega_{i}=\Omega$

$$
\begin{aligned}
\frac{1}{2} \sum_{i=1}^{N} \sum_{t=1}^{T} \operatorname{vec}\left((\Omega)^{-1}-(\Omega)^{-1} u_{i, t} u_{i, k}^{\prime}(\Omega)^{-1}\right)^{\prime} D_{k} & =0 \Longleftrightarrow \\
\frac{1}{2} \sum_{i=1}^{N} \sum_{t=1}^{T} D_{k}\left((\Omega)^{-1} \otimes(\Omega)^{-1}\right) \operatorname{vec}\left(\Omega-u_{i, t} u_{i, t}^{\prime}\right) & =0 \Longleftrightarrow \\
\frac{1}{2} \sum_{i=1}^{N} \sum_{t=1}^{T} D_{k}^{\prime}\left((\Omega)^{-1} \otimes(\Omega)^{-1}\right) D_{k} \operatorname{vech}\left(\Omega-u_{i, t} u_{i, t}^{\prime}\right) & =0 .
\end{aligned}
$$
Observe that since $D_{k}^{\prime}\left((\Omega)^{-1} \otimes(\Omega)^{-1}\right) D_{k}$ is invertible it follows that this is equivalent
with

$$
\operatorname{vech}(\Omega)=\frac{1}{N T} \sum_{i=1}^{N} \sum_{t=1}^{T} \operatorname{vech}\left(u_{i, t} u_{i, t}^{\prime}\right)
$$


For the model with individual covariance matrices $\Omega_{i}=\Omega_{i}$ we obtain using

$$
\frac{1}{2} \sum_{i=1}^{N} \sum_{t=1}^{T} \operatorname{vec}\left(\Omega_{i}^{-1}-\Omega_{i}^{-1} u_{i, t} u_{i, t}^{\prime} \Omega_{i}^{-1}\right)^{\prime}\left(e_{i, N}^{\prime} \otimes D_{k}\right)=0
$$

in a similar fashion

$$
\begin{aligned}
\theta^{i} & =\left(\operatorname{vech}\left(\Omega_{1}\right)^{\prime}, \ldots, \operatorname{vech}\left(\Omega_{N}\right)^{\prime}\right)^{\prime} \\
& =\left(\frac{1}{T} \sum_{t=1}^{T} \operatorname{vech}\left(u_{1, t} u_{1, t}^{\prime}\right), \ldots \frac{1}{T} \sum_{t=1}^{T} \operatorname{vech}\left(u_{N, t} u_{N, t}^{\prime}\right)\right)^{\prime} .
\end{aligned}
$$

The estimators for the parameters of $\Omega^{s b}$ and $\Omega^{s w}$ are more complicated. First we solve the equations for the parameters $w\left(\Sigma_{i}\right)$ of $\Omega_{i}=\Gamma \Sigma_{i}^{2} \Gamma^{\prime}$. The following equalities are equivalent.

$$
\begin{aligned}
\sum_{t=1}^{T}\left(\operatorname{vec}\left(\Omega_{i}^{-1}-\Omega_{i}^{-1} u_{i, t} u_{i, t}^{\prime} \Omega_{i}^{-1}\right)\right)^{\prime} N_{k}\left(\Gamma \otimes \Gamma \Sigma_{i}^{\prime}\right) \Psi_{k}^{\prime} & =0 \\
\sum_{t=1}^{T} \Psi_{k}\left(\Gamma^{\prime} \otimes \Sigma_{i} \Gamma^{\prime}\right) \operatorname{vec}\left(\Omega_{i}^{-1}-\Omega_{i}^{-1} u_{i, t} u_{i, t}^{\prime} \Omega_{i}^{-1}\right) & =0 \\
\sum_{t=1}^{T} \Psi_{k} \operatorname{vec}\left(I_{k}-\Gamma^{-1} u_{i, t} u_{i, t}^{\prime} T^{-1 /} \Sigma_{i}^{-2}\right) & =0 \\
\sum_{t=1}^{T} \Psi_{k}\left(\Sigma_{i}^{-2} \otimes I\right) \operatorname{vec}\left(\Sigma_{i}^{2}-\Gamma^{-1} u_{i, t} u_{i, t}^{\prime} \Gamma^{-1 \prime}\right) & =0 .
\end{aligned}
$$

For the first equality we use the fact that when $N_{k}$ operates on the vec of a symmetric matrix it does not affect the vec of that matrix. From the last equation we note that due to the diagonality of $\Sigma_{i}^{-2}$ we have $\Psi_{k}\left(\Sigma_{i}^{-2} \otimes I\right)=\Sigma_{i}^{-2} \Psi_{k}$. Using the nonsingularity of $\Sigma_{i}^{-2}$ we obtain

$$
\Psi_{k} \operatorname{vec}\left(\Sigma_{i}^{2}\right)=\frac{1}{T} \sum_{t=1}^{T} \Psi_{k} \operatorname{vec}\left(\Gamma^{-1} u_{i, t} u_{i, t}^{\prime} \Gamma^{-1 \prime}\right) .
$$

For the parameters $v(\Gamma)$ of $\Omega^{s b}$ we derive the first order conditions using the following equivalent equations

$$
\begin{aligned}
\sum_{i=1}^{N} \sum_{t=1}^{T}\left(\operatorname{vec}\left(\Omega_{i}^{-1}-\Omega_{i}^{-1} u_{i, t} u_{i, t}^{\prime} \Omega_{i}^{-1}\right)\right)^{\prime} N_{k}\left(\Gamma \Sigma_{i}^{2} \otimes I_{k}\right) \tilde{L}_{k}^{\prime} & =0 \\
\sum_{i=1}^{N} \sum_{t=1}^{T} \tilde{L}_{k}\left(\Sigma_{i}^{2} \Gamma^{\prime} \otimes I_{k}\right) \operatorname{vec}\left(\Omega_{i}^{-1}-\Omega_{i}^{-1} u_{i, t} u_{i, t}^{\prime} \Omega_{i}^{-1}\right) & =0 \\
\sum_{i=1}^{N} \sum_{t=1}^{T} \tilde{L}_{k} \operatorname{vec}\left(\Gamma^{-1 \prime}-\Gamma^{-1 /} \Sigma_{i}^{-2} \Gamma^{-1} u_{i, t} i u_{i, t}^{\prime} \Gamma^{-1 \prime}\right) & =0
\end{aligned}
$$




$$
\begin{aligned}
\sum_{i=1}^{N} \sum_{t=1}^{T} \tilde{L}_{k}\left(\Gamma^{-1} \otimes \Gamma^{-1}\right) \text { vec }\left(\Gamma^{\prime}-\Sigma_{i}^{-2} \Gamma^{-1} u_{i, t} u_{i, t}^{\prime}\right) & =0 \\
\sum_{i=1}^{N} \sum_{t=1}^{T} \tilde{L}_{k}\left(\Gamma^{-1} \otimes \Gamma^{-1}\right) \tilde{L}_{k}^{\prime} \tilde{L}_{k} \operatorname{vec}\left(\Gamma^{\prime}-\Sigma_{i}^{-2} \Gamma^{-1} u_{i_{i}, t} u_{i, t}^{\prime}\right) & =0 .
\end{aligned}
$$

These can be restated as

$$
\begin{aligned}
\sum_{i=1}^{N} \sum_{t=1}^{T} \tilde{L}_{k} \operatorname{vec}\left(\Gamma^{-1}\right) & =\sum_{i=1}^{N} \sum_{t=1}^{T} \tilde{L}_{k} \operatorname{vec}\left(\Gamma^{-1} \Sigma_{i}^{-2} \Gamma^{-1} u_{i, t} u_{i, t}^{\prime} \Gamma^{-1 \prime}\right) \\
\tilde{L}_{k} \operatorname{vec}\left(\Gamma^{-1}\right) & =\frac{1}{N T} \sum_{i=1}^{N} \sum_{t=1}^{T} \tilde{L}_{k} \operatorname{vec}\left(\Gamma^{-1} \Sigma_{i}^{-2} \Gamma^{-1} u_{i, t} u_{i, t}^{\prime} \Gamma^{-1 \prime}\right) .
\end{aligned}
$$

The conditions (4.55) and (4.56) have to be solved jointly with the first order conditions for $\mu_{i}$ and $\Phi$.

For the parameters $v(\Gamma)$ of $\Omega_{i}^{s w}=\Sigma_{i} \Gamma \Gamma^{\prime} \Sigma_{i}$ we have the following equivalent equalities

$$
\begin{aligned}
& \sum_{i=1}^{N} \sum_{t=1}^{T}\left(\operatorname{vec}\left(\Omega^{-1}-\Omega^{-1} u_{i, t} u_{i_{i}, t}^{\prime} \Omega^{-1}\right)\right)^{\prime} N_{K}\left(\left(\Sigma_{i} \Gamma \otimes \Sigma_{i}\right)\right) \ddot{L}_{k}^{\prime}=0 \\
& \sum_{i=1}^{N} \sum_{t=1}^{T} \tilde{L}_{k}\left(\Gamma^{\prime} \Sigma_{i} \otimes \Sigma_{i}\right) \operatorname{vec}\left(\Omega^{-1}-\Omega^{-1} u_{i, t} u_{i, t}^{\prime} \Omega^{-1}\right)=0 \\
& \sum_{i=1}^{N} \sum_{t=1}^{T \prime} \tilde{L}_{k} \operatorname{vec}\left(\Gamma^{-1 \prime}-\Gamma^{-1} \Gamma^{-1} \Sigma_{i}^{-1} u_{i, t} u_{i, t}^{\prime} \Sigma_{i}^{-1} \Gamma^{-1 \prime}\right)=0 \\
& \sum_{i=1}^{N} \sum_{t=1}^{T^{\prime}} \widetilde{L}_{k}\left(\Gamma^{-1} \otimes \Gamma^{-1 \prime}\right) \operatorname{vec}\left(\Gamma^{*}-\Gamma^{-1} \Sigma_{i}^{-1} u_{i, t} u_{i_{0} t}^{\prime} \Sigma_{i}^{-1}\right)=0 \\
& \sum_{i=1}^{N} \sum_{t=1}^{T_{t}} \operatorname{vec}\left(\Gamma^{\prime \prime}-\Gamma^{-1} \Sigma_{i}^{-1} u_{i, t} u_{i, t}^{\prime} \Sigma_{i}^{-1}\right) \tilde{L}_{k}^{\prime} \tilde{L}_{k}\left(\Gamma^{-1^{\prime}} \otimes \Gamma^{-1}\right) \tilde{L}_{k}^{\prime}=0 \\
& \sum_{i=1}^{N} \sum_{t=1}^{T_{i}} \hat{L}_{k} \operatorname{vec}\left(\Gamma^{\prime}-\Gamma^{-1} \Sigma_{i}^{-1} u_{i, t} u_{i, t}^{\prime} \Sigma_{i}^{-1}\right)=0 \\
& \sum_{i=1}^{N} \sum_{t=1}^{T} \tilde{L}_{k} \operatorname{vec}\left(I-\Gamma^{-1} \Sigma_{i}^{-1} u_{i, t} \cdot u_{i, t}^{\prime} \Sigma_{i}^{-1} \Gamma^{-1^{\prime}}\right)=0 .
\end{aligned}
$$

We car restate this using the following equivalent equations

$$
\begin{aligned}
\tilde{L}_{k} \operatorname{vec}\left(I_{k}\right)= & \frac{1}{N T} \sum_{i=1}^{N} \sum_{t=1}^{T \prime} \tilde{L}_{k}\left(\Gamma^{-1} \otimes \Gamma^{-1^{t}}\right) \\
& \times \operatorname{vec}\left(\Gamma^{-1} \Sigma_{i}^{-1} u_{i, t} u_{i, t}^{\prime} \Sigma_{i}^{-1} \Gamma^{-1}\right) \\
\operatorname{vec}\left(I_{k}\right) \tilde{L}_{k}^{\prime \prime}= & \frac{1}{N T} \sum_{i=1}^{N} \sum_{i=1}^{T \prime} \operatorname{vec}\left(\Sigma_{i}^{-1} u_{i, t} u_{i, t}^{\prime} \Sigma_{i}^{-1}\right)^{\prime \prime}
\end{aligned}
$$




$$
\begin{aligned}
\operatorname{vec}\left(I_{k}\right)^{\prime} \tilde{L}_{k}^{\prime} \tilde{L}_{k}\left(\Gamma^{\prime} \otimes \Gamma\right) \tilde{L}_{k}^{\prime} & =\frac{1}{N T} \sum_{i=1}^{N} \sum_{t=1}^{T} \tilde{L}_{i k}^{\prime} \tilde{L}_{k}\left(\Gamma^{-1^{\prime}} \otimes \Gamma^{-1}\right) \tilde{L}_{k}^{\prime}\left(\Sigma_{i}^{-1} u_{i, t} u_{i, t}^{\prime} \Sigma_{i}^{-1}\right)^{\prime} \tilde{L}_{k}^{\prime} \\
\hat{L}_{k} \operatorname{vec}\left(\Gamma^{\prime}\right)= & \frac{1}{N T} \sum_{i=1}^{N} \sum_{t=1}^{T} \tilde{L}_{k} \operatorname{vec}\left(\Sigma_{i}^{-1} u_{i, t} u_{i, t}^{\prime} \Sigma_{i}^{-1}\right) .
\end{aligned}
$$

For the parameters $w\left(\Sigma_{i}\right)$ we have the equations

$$
\begin{aligned}
\sum_{i=1}^{N} \sum_{t=1}^{T}\left(\operatorname{vec}\left(\Omega^{-1}-\Omega^{-1} u_{i, t} u_{i, t}^{\prime} \Omega^{-1}\right)\right)^{\prime} N_{K}\left(\Sigma_{i} \Gamma \Gamma^{\prime} \otimes I_{k}\right) \Psi_{k}^{\prime} & =0 \\
\sum_{i=1}^{N} \sum_{t=1}^{T} \Psi_{k}\left(\Gamma^{\prime} \Gamma \Sigma_{i} \otimes I_{k}\right) \operatorname{vec}\left(\Omega^{-1}-\Omega^{-1} u_{i, t} u_{i, t}^{\prime} \Omega^{-1}\right) & =0 \\
\sum_{i=1}^{N} \sum_{t=1}^{T} \Psi_{k} \operatorname{vec}\left(\Sigma_{i}^{-1}-\Omega_{i}^{-1} u_{i, t} u_{i, t}^{\prime} \Sigma_{i}^{-1}\right) & =0 \\
\sum_{i=1}^{N} \sum_{t=1}^{T} \Psi_{k} \operatorname{vec}\left(\Sigma_{i}^{-1}-\Sigma_{i}^{-1} \Gamma^{-1} \Gamma^{-1} \Sigma_{i}^{-1} u_{i, t} u_{i, t}^{\prime} \Sigma_{i}^{-1}\right) & =0 \\
\sum_{i=1}^{N} \sum_{t=1}^{T} \Psi_{k}\left(\Sigma_{i}^{-1} \otimes I\right) \operatorname{vec}\left(I-\Sigma_{i}^{-1} \Gamma^{\prime-1} \Gamma^{-1} \Sigma_{i}^{-1} u_{i, t} u_{i, t}^{\prime}\right) & =0
\end{aligned}
$$

which are equivalent. Now observe that $\Psi_{k}\left(\Sigma_{i}^{-1} \otimes I\right)=\Sigma_{i}^{-1} \Psi_{k}$ and that $\Sigma_{i}^{-1}$ is invertible. This gives

$$
\Psi_{k} \operatorname{vec}(I)=\frac{1}{N T} \sum_{i=1}^{N} \sum_{t=1}^{T} \Psi_{k} \operatorname{vec}\left(\Sigma_{i}^{-1} \Gamma^{\prime-1} \Gamma^{-1} \Sigma_{i}^{-1} u_{i, t} u_{i, t}^{\prime}\right) .
$$

Proof. (Proof of Theorem 17) From Theorem 15 and Lemma 20 it directly follows that (4.40) and (4.41) hold. Introducing the parameter vectors $\gamma=v(\Gamma)$ and $\sigma=$ $\left(w\left(\Sigma_{1}\right)^{\prime}, \ldots, w\left(\Sigma_{N}\right)^{\prime}\right)^{\prime}$ we have $\theta=(\gamma, \sigma)^{\prime}$ and we can partition the information matrix into

$$
I_{(\theta)}=\left[\begin{array}{cc}
I_{\gamma \gamma^{\prime}} & I_{\gamma \sigma^{\prime}} \\
I_{\sigma \gamma^{\prime}} & I_{\sigma \sigma^{\prime}}
\end{array}\right]
$$

Using the following observations: $N_{k} K_{k k}=N_{k}=N_{k}^{\prime}, N_{k}(A \otimes A) N_{k}=(A \otimes A) N_{k}$, $\Omega_{i}^{s b}=\Gamma \Sigma_{i}^{2} \Gamma^{\prime},\left(\Omega_{i}^{s b}\right)^{-1}=\Gamma^{\gamma-1} \Sigma_{i}^{-2} \Gamma^{-1}$ and $K_{k k}(A \otimes B)=(B \otimes A) K_{k k}$ we obtain for the parameter vector

$$
\begin{aligned}
I_{\gamma \gamma^{\prime}} & =\frac{2}{N} \sum_{i=1}^{N}\left(\left(\Gamma \Sigma_{i}^{2} \otimes I_{k}\right) \tilde{L}_{k}^{\prime}\right)^{\prime}\left(\Omega_{i}^{-1} \otimes \Omega_{i}^{-1}\right) N_{k}\left(\Gamma \Sigma_{i}^{2} \otimes I_{k}\right) \tilde{L}_{k}^{\prime} \\
& =\frac{2}{N} \sum_{i=1}^{N} \tilde{L}_{K}\left(\Gamma^{-1} \otimes \Omega_{i}^{-1}\right) N_{k}\left(\Gamma \Sigma_{i}^{2} \otimes I_{k}\right) \tilde{L}_{k}^{\prime}
\end{aligned}
$$




$$
\begin{aligned}
& =\frac{1}{N} \sum_{i=1}^{N} \tilde{L}_{K}\left(\Sigma_{i}^{2} \otimes \Omega_{i}^{-1}\right) \tilde{L}_{k}^{\prime}+\tilde{L}_{K}\left(\Gamma^{-1} \otimes \Omega_{i}^{-1}\right) K_{k k}\left(\Gamma \Sigma_{i}^{2} \otimes I_{k}\right) \tilde{L}_{k}^{\prime} \\
& =\frac{1}{N} \sum_{i=1}^{N} \tilde{L}_{K}\left(\Sigma_{i}^{2} \otimes \Omega_{i}^{-1}\right) \tilde{L}_{k}^{\prime}+\tilde{L}_{K}\left(\Gamma^{-1} \otimes \Gamma^{-1^{\prime}}\right) K_{k k} \tilde{L}_{k}^{\prime} \\
& =\frac{1}{N} \sum_{i=1}^{N} \tilde{L}_{K}\left(\Sigma_{i}^{2} \otimes \Omega_{i}^{-1}\right) \tilde{L}_{k}^{\prime}+\tilde{L}_{k} \widetilde{K}_{k k} \tilde{L}_{k}^{\prime} \tilde{L}_{k}\left(\Gamma^{-1^{\prime}} \otimes \Gamma\right) \tilde{L}_{k}^{\prime} .
\end{aligned}
$$

For the parameter vector $\sigma$ we obtain

$$
\begin{aligned}
I_{\sigma \sigma^{\prime}} & =\frac{2}{N} \sum_{i=1}^{N}\left(e_{i, N}^{\prime} \otimes\left(\Gamma \otimes \Gamma \Sigma_{i}\right) \Psi_{k}^{\prime}\right)^{\prime}\left(\Omega_{i}^{-1} \otimes \Omega_{i}^{-1}\right) N_{k}\left(e_{i, N}^{\prime} \otimes\left(\Gamma \otimes \Gamma \Sigma_{i}\right) \Psi_{k}^{\prime}\right) \\
& =\frac{2}{N} \sum_{i=1}^{N}\left(e_{i, N} \otimes \Psi_{k}\left(\Gamma^{\prime} \otimes \Sigma_{i} \Gamma^{\prime}\right)\right)\left(\Omega_{i}^{-1} \otimes \Omega_{i}^{-1}\right) N_{k}\left(e_{i, N}^{\prime} \otimes\left(\Gamma \otimes \Gamma \Sigma_{i}\right) \Psi_{k}^{\prime}\right) \\
& =\frac{2}{N} \sum_{i=1}^{N}\left(e_{i, N} e_{i, N}^{\prime} \otimes \Psi_{k}\left(\Gamma^{\prime} \Omega_{i}^{-1} \otimes \Sigma_{i} \Gamma^{\prime} \Omega_{i}^{-1}\right) N_{k}\left(\Gamma \otimes \Gamma \Sigma_{i}\right) \Psi_{k}^{\prime}\right) \\
& =\frac{1}{N} \sum_{i=1}^{N}\left(e_{i, N} e_{i, N}^{\prime} \otimes \Psi_{k}\left(\Sigma_{i}^{-2} \otimes I_{k}\right) \Psi_{k}^{\prime}+\Psi_{k}\left(\Sigma_{i} \otimes \Sigma_{i}^{-1}\right) K_{k k} \Psi_{k}^{\prime}\right)
\end{aligned}
$$

For the cross term we obtain

$$
\begin{aligned}
I_{\sigma \gamma^{\prime}} & =\frac{2}{N} \sum_{i=1}^{N}\left(e_{i, N}^{\prime} \otimes\left(\Gamma \otimes \Gamma \Sigma_{i}\right) \Psi_{k}^{\prime}\right)^{\prime}\left(\Omega_{i}^{-1} \otimes \Omega_{i}^{-1}\right) N_{k}\left(\Gamma \Sigma_{i}^{2} \otimes I_{k}\right) \tilde{L}_{k}^{\prime} \\
& =\frac{2}{N} \sum_{i=1}^{N}\left(e_{i, N} \otimes \Psi_{k}\left(\Gamma^{\prime} \otimes \Sigma_{i} \Gamma^{\prime}\right)\left(\Omega_{i}^{-1} \otimes \Omega_{i}^{-1}\right) N_{k}\left(\Gamma \Sigma_{i}^{2} \otimes I_{k}\right) \widetilde{L}_{k}^{\prime}\right. \\
& =\frac{2}{N} \sum_{i=1}^{N}\left(e_{i, N} \otimes \Psi_{k}\left(\Gamma^{\prime} \otimes \Sigma_{i} \Gamma^{\prime}\right) N_{k}\left(\Gamma^{\prime-1} \otimes \Omega_{i}^{-1}\right) \tilde{L}_{k}^{\prime}\right. \\
& =\frac{2}{N} \sum_{i=1}^{N}\left(e_{i, N} \otimes \Psi_{k}\left(\Gamma^{\prime} \otimes \Sigma_{i} \Gamma^{\prime}\right) N_{k}\left(\Gamma^{\prime-1} \otimes \Omega_{i}^{-1}\right) \tilde{L}_{k}^{\prime}\right) \\
& =\frac{1}{N} \sum_{i=1}^{N}\left(e_{i, N} \otimes \Psi_{k}\left(\Gamma^{\prime} \otimes \Sigma_{i}^{-1} \Gamma^{-1}\right) \tilde{L}_{k}^{\prime}+\Psi_{k}\left(\Sigma_{i}^{-1} \Gamma^{-1} \otimes \Sigma_{i}\right) K_{k k} \tilde{L}_{k k}^{\prime}\right)
\end{aligned}
$$

For $\Omega_{i}^{s w}=\Sigma_{i} \Gamma \Gamma^{\prime} \Sigma_{i} \Omega_{i}^{s w-1}=\Sigma_{i}^{-1} \Gamma^{-1} \Gamma^{-1} \Sigma_{i}^{-1}$ we have in a similar way

$$
\begin{aligned}
I_{\gamma \gamma^{\prime}} & =\frac{2}{N} \sum_{i=1}^{N}\left(\left(\Sigma_{i} \Gamma \otimes \Sigma_{i}\right) \tilde{L}_{k}^{\prime \prime}\right)^{\prime}\left(\Omega_{i}^{-1} \otimes \Omega_{i}^{-1}\right) N_{k}\left(\Sigma_{i} \Gamma \otimes \Sigma_{i}\right) \tilde{L}_{k}^{\prime} \\
& =\frac{2}{N} \sum_{i=1}^{N} \tilde{L}_{K}\left(\Gamma^{-1} \Sigma_{i}^{-1} \otimes \Gamma^{-1} \Gamma^{-1} \Sigma_{i}^{-1}\right) N_{k}\left(\Sigma_{i} \Gamma \otimes \Sigma_{i}\right) \tilde{L}_{k}^{\prime}
\end{aligned}
$$




$$
\begin{aligned}
& =\frac{1}{N} \sum_{k=1}^{N} \tilde{L}_{K}\left(I \otimes \Gamma^{-1 \prime} \Gamma^{-1}\right) \tilde{L}_{k}^{\prime}+\tilde{L}_{K}\left(\Gamma^{-1} \otimes \Gamma^{-1 \prime}\right) K_{k k} \widetilde{L}_{k}^{\prime} \\
& =\widetilde{L}_{K}\left(I \otimes \Gamma^{-1 /} \Gamma^{-1}\right) \widetilde{L}_{k}^{\prime}+\tilde{L}_{K}\left(\Gamma^{-1} \otimes \Gamma^{-1 \prime}\right) K_{k k} \tilde{L}_{k}^{\prime} .
\end{aligned}
$$

For $\sigma$ we have

$$
\begin{aligned}
I_{\sigma \sigma^{\prime}} & =\frac{2}{N} \sum_{i=1}^{N}\left(e_{i, N}^{\prime} \otimes\left(\Sigma_{i} \Gamma \Gamma^{\prime} \otimes I_{k}\right) \Psi_{k}^{\prime}\right)^{\prime}\left(\Omega_{i}^{-1} \otimes \Omega_{i}^{-1}\right) N_{k}\left(e_{i, N}^{\prime} \otimes\left(\Sigma_{i} \Gamma \Gamma^{\prime} \otimes I_{k}\right) \Psi_{k}^{\prime}\right) \\
& =\frac{2}{N} \sum_{i=1}^{N}\left(e_{i, N} e_{i, N}^{\prime} \otimes \Psi_{k}\left(\Gamma^{\prime} \Gamma \Sigma_{i} \otimes I_{k}\right)\left(\Omega_{i}^{-1} \otimes \Omega_{i}^{-1}\right) N_{k}\left(\Sigma_{i} \Gamma \Gamma^{\prime} \otimes I_{k}\right) \Psi_{k}^{\prime}\right) \\
& =\frac{2}{N} \sum_{i=1}^{N}\left(e_{i, N} e_{i, N}^{\prime} \otimes \Psi_{k}\left(\Sigma_{i}^{-1} \otimes \Omega_{i}^{-1}\right) N_{k}\left(\Sigma_{i} \Gamma \Gamma^{\prime} \otimes I_{k}\right) \Psi_{k}^{\prime}\right) \\
& =\frac{1}{N} \sum_{i=1}^{N}\left(e_{i, N} e_{i, N}^{\prime} \otimes \Psi_{k}\left(\Gamma \Gamma^{\prime} \otimes \Omega_{i}^{-1}\right) \Psi_{k}^{\prime}+\Psi_{k}\left(\Sigma_{i}^{-1} \otimes \Sigma_{i}^{-1}\right) K_{k k} \Psi_{k}^{\prime}\right)
\end{aligned}
$$

And finally we have

$$
\begin{aligned}
I_{\sigma \gamma^{\prime}} & =\frac{2}{N} \sum_{i=1}^{N}\left(e_{i, N}^{\prime} \otimes\left(\Sigma_{i} \Gamma \Gamma^{\prime} \otimes I_{k}\right) \Psi_{k}^{\prime}\right)^{\prime}\left(\Omega_{i}^{-1} \otimes \Omega_{i}^{-1}\right) N_{k}\left(\Sigma_{i} \Gamma \otimes \Sigma_{i}\right) \widetilde{L}_{k}^{\prime} \\
& =\frac{2}{N} \sum_{i=1}^{N}\left(e_{i, N} \otimes \Psi_{k}\left(\Gamma^{\prime} \Gamma \Sigma_{i} \otimes I_{k}\right)\left(\Omega_{i}^{-1} \otimes \Omega_{i}^{-1}\right) N_{k}\left(\Sigma_{i} \Gamma \otimes \Sigma_{i}\right) \tilde{L}_{k}^{\prime}\right) \\
& =\frac{2}{N} \sum_{i=1}^{N}\left(e_{i, N} \otimes \Psi_{k}\left(\Sigma_{i}^{-1} \otimes \Omega_{i}^{-1}\right) N_{k}\left(\Sigma_{i} \Gamma \otimes \Sigma_{i}\right) \tilde{L}_{k}^{\prime}\right) \\
& =\frac{1}{N} \sum_{i=1}^{N}\left(e_{i, N} \otimes \Psi_{k}\left(\Gamma \otimes \Sigma_{i}^{-1} \Gamma^{-1 /} \Gamma^{-1}\right) \tilde{L}_{k}^{\prime}+\Psi_{k}\left(\Sigma_{i}^{-1} \otimes \Omega_{i}^{-1}\right)\left(\Sigma_{i} \otimes \Sigma_{i} \Gamma\right) K_{k} \tilde{L}_{k}^{\prime}\right) \\
& =\frac{1}{N} \sum_{i=1}^{N}\left(e_{i, N} \otimes \Psi_{k}\left(\Gamma \otimes \Sigma_{i}^{-1} \Gamma^{-1 /} \Gamma^{-1}\right) \tilde{L}_{k}^{\prime}+\Psi_{k}\left(I_{k} \otimes \Sigma_{i}^{-1} \Gamma^{-1}\right) K_{k} \tilde{L}_{k}^{\prime}\right)
\end{aligned}
$$




\section{Chapter 5}

\section{Non-linear Dynamic Panel Data Models: A multi-country Application to Saving, Openness and Growth}

\subsection{Introduction}

This chapter brings together two important parts of the empirical literature on the determinants of economic growth, namely growth and openness to trade on the one hand and growth and saving on the other. A lot of empirical work has been done on the relationship between growth and various measures of openness to trade (Harrison (1996), Edwards (1993), Jorgenson and Ho (1993), Balassa (1985), (1989), Esfahani (1991), Quah and Rauch (1990)). Most studies show a positive relationship between most measures of openness and growth. The results, however, are often statistically insignificant and fragile to changes in the econometric model or the data set (Levine and Renelt (1992), Edwards (1992)). For example, in Anne Harrison's study only three of the six measures of openness are significant at the five percent level (Table 6, p. 434).

There exists an equally impressive work on the relationship between growth and saving (Maddison (1992), Carroll and Weil (1993), Bosworth (1993), Gupta and Islam (1983)). The recent literature on the topic has found that countries with higher saving or investment rates had significantly higher growth rates, a result that sensitivity tests show to be fairly robust (Levine and Renelt (1992)). Recent theoretical results from the literature on trade and endogenous growth point out that certain model parameters linked to household behavior play a key role in the interaction between growth and openness (Feenstra (1996), Osang and Pereira (1996a), (1996b)). Feenstra shows that trade can increase or decrease a country's growth rate depending on the value of the instantaneous elasticity of substitution, while Osang and Pereira point out that there exists a threshold level of the intertemporal elasticity of substitution that separates growth-enhancing from growth-reducing regimes of increased openness to trade. ${ }^{1}$ Since the intertemporal elastic-

\footnotetext{
The mechanism through which openness affects growth is as follows. International differences in
} 
ity of substitution is a key parameter for saving in the endogenous growth literature, it seems natural to test the hypothesis that the relation between a country's openness and its growth rate changes once the saving rate exceeds a certain endogenously determined threshold level. If the hypothesis cannot be rejected by the data, one could argue that the weak link between openness and growth found in the literature may simply be the result of a misspecified model.

In order to test the above hypothesis, we estimate a dynamic model where we simultaneously estimate the benchmark value of the saving rate and the other parameters of the model. We use data on 58 countries during the period 1962-1987. The main finding of the chapter is that saving rates matter for the link between openness and growth. There is a significantly positive effect of openness on output for years with above benchmark saving rates. This is in contrast with an insignificant effect for years with saving rates below the benchmark value.

Modelling a threshold raises some interesting problems concerning the underlying econometric theory since the estimation model is not well defined in the case of a nonexistent threshold level. With regard to this problem this chapter introduces a new simple test for the existence of a threshold. The test is based on results derived by Bierens and Ploberger (1995). The test is easier to calculate than for instance the test for a threshold by Hansen (1995), (1996). It might also be used as alternative to the tests by Andrews et all. (1996) and Andrews and Ploberger (1994).

This chapter extends the existing empirical literature in two ways. First, we estimate the non-linear relationship between saving rates, openness to trade, and growth which is absent in the standard growth literature cited above. Second, by using a dynamic model instead of a simple cross-section analysis, we can allow the effect of saving on the relationship between openness and growth to work both in the cross section dimension (across countries) as well as across time for each individual country. This is important since the cross section approach ignores the effects through time of changes in openness policies This was shown by Harrison (1996).

The remainder of this chapter is organized as follows. Section two describes the estimated model in detail. We discuss the econometric methodology necessary for estimation and testing of thresholds in panel data sets in section three. Section four describes the data, while section five contains the empirical results. Section six concludes the chapter. In three appendices we give proofs and additional results.

\subsection{The empirical model}

The recent theoretical literature on growth ${ }^{2}$ in open economies suggests that GDP growth depends on the following parameters:

preferences and/or technologies lead to different steady state growth rates across countries. Assuming balanced trade and complete specialization, increasing the volume of trade in both countries (e.g. due to lower trade barriers or changes in consumer preferences) induces changes in the terms of trade. In this situation it is most likely that the conntry with the weak attitude toward saving will experience an improvement in its terms of trade and, in turn, a decline in output growth, while the country with the strong saving performance will experience the opposite effects.

${ }^{2}$ See, for example Lee (1995), Rivera-Batiz and Romer (1991) and Turnovsky (1996). 
- Technology parameters such as total factor productivity, $A$, and scale elasticities; $\alpha$.

- Taste parameters such as the intertemporal elasticity of substitution, $\sigma$, and the discount rate, $\rho$.

- Trade policy parameters, $\tau$, measuring a country's tariff and non-tariff barriers to trade.

The relationship can be expressed as follows

$$
\text { growth }=f(A, \alpha, \sigma, \rho, \tau) .
$$

Unfortunately in most cases we lack data that directly measure these parameters, especially over time. It is thus common in the literature to approximate these parameters with data that are available both across countries and over time.

Taste parameters reflect a country's willingness to postpone current consumption and thus determine the domestic supply of financial capital. Advanced production technologies are intensive in both physical and human capital and correspond to high levels of total factor productivity. Advanced production technologies are thus a major factor behind the domestic demand for financial capital. It seems reasonable to use the domestic saving rate of a country as a proxy for both sufficient supply and adequate demand in the market for financial capital as international capital mobility is debatable (see Gordon and Bovenberg (1996)).

Trade policy parameters can be approximated either directly through some index of trade liberalization using country sources on trade barriers (see, for example, Thomas et al. (1991)) or indirectly through measures such as the blackmarket premium in the currency market or an index measuring price distortions for consumption goods. ${ }^{3} \mathrm{~A}$ widely used indirect measure for trade barriers is the ratio of exports plus imports to GDP. This proxy has the advantage that it is available for many countries for at least three decades. It is also relatively free of different definitions and data collection techniques between countries. Furthermore, Harrison (1996) shows that it has the highest correlation coefficient with trade reform compared to all other indirect openness measures. (Table 2, p. 429). Its most severe disadvantage is that its value does not depend on trade barriers alone but also on country size or foreign direct investment. Nevertheless, we will use the share of trade in GDP as our proxy for a country's openness to trade.

Using the above variables leaves us with a model of the form

$$
\text { growth }=f(s, 0)
$$

where $s$ indicates the savings ratio, and $o$ indicates the level of openness. In our case the savings ratio is the nominal gross domestic saving rate (Gross Domestic Saving = GDP - Consumption) to nominal gross domestic product while openness is the ratio of volume of trade to gross domestic product. The technology parameters are assumed to be

\footnotetext{
${ }^{3}$ See Harrison (1996) for other indirect measures of trade barriers as well as a detailed dicussion of measurement problems associated with both direct and indirect proxies of trade barriers.
} 
incorporated in the function $f$. Note that (5.2) comes very close to the usual assumptions in endogenous growth theory where output growth is usually modelled as a function of the savings with the form of the function depending on the technology and taste parameters.

As most of the relations given above are assumed to hold in equilibrium in practice the equilibrium will not be reached and be affected through shocks. Therefore it is also important to model dymamics. For this reason and the fact that the functional form of $f$ in (5.2) is unknown we use a linear first order vector autoregressive model as a first order approximation.

We are interpreting the savings ratio as an approximation for the taste parameters and the volume of trade as an approximation for trade policy or openness. We assume the technology parameters to be incorporated in the intercept and to be equal for every country. This last assumption is rather restrictive but at the moment there is no technique available to determine the endogenous threshold in a. real panel data context with individual effects.

The above considerations lead to the following unrestricted VAR(1)

$$
X_{i t}=c+A_{1} X_{i, t-1}+\varepsilon_{i t},
$$

with $X_{i, t}=\left(O_{i t}, S_{i, t}, \Delta Y_{i t}\right)^{\prime}$ where

- $\Delta Y_{i t}$ denotes GDP growth of country $i$ at time $t,\left(\Delta Y_{i t}=\log \left(Y_{i t}\right)-\log \left(Y_{i t-1}\right)\right)$

- $S_{i t}$ denotes the gross domestic saving rate of country $i$ at time $t$,

- $O_{i t}$ denotes the log ratio of imports plus exports to output of country $i$ at time $t$.

We assume that the intercept $c$ catches the technology effect and the disturbance term $\varepsilon_{i t}$ to be independent Gaussian with $E\left(\varepsilon_{i t}\right)=0$, and covariance $E\left(\varepsilon_{i t} \varepsilon_{i t}^{\prime}\right)=\Omega_{i}$.

Because we are also interested in the instantaneous effects and we assume these effects to be the same for each individual country we rewrite (5.3) in the following structural form

$$
X_{i, t}=\mu+B_{0} X_{i, t}+B_{1} X_{i, t-1}+u_{i t}
$$

where $u_{i t}$ is again independent Gaussian with zero mean but with diagonal covariance matrix $E\left(u_{i t} u_{i t}^{\prime}\right)=\operatorname{diag}\left(\sigma_{i, 1}^{2}, \sigma_{i, 2}^{2}, \sigma_{i, 3}^{2}\right) \equiv \Lambda_{i}$, where $\Omega_{i}=\Gamma \Lambda_{i} \Gamma^{\prime}$ and $\Gamma$ is lower triangular with ones on the main diagonal. We then have $\mu=\Gamma^{-1} c_{y} B_{0}=\left(I-\Gamma^{-1}\right)$ and $B_{1}=\Gamma^{-1} A_{1}$. Observe that $B_{0}$ is lower triangular with zeros on the main diagonal ${ }^{4}$.

To test the hypothesis the relation between openness and GDP growth changes once that the saving rate exceeds a certain level we generallize the above model to the following model:

$$
X_{i, t}=\mu+B_{0} X_{i, t}+B_{1} X_{i, t-1}+\left(\bar{\mu}+\bar{B}_{0} X_{i, t}+\bar{B}_{1} X_{i, t-1}\right) I\left(S_{i, t-2}>\gamma\right)+w_{i t}
$$

where $I\left(S_{i, t-2}>\gamma\right)$ is the indicator function which equals one when the inequality holds and zero otherwise. The unknown threshold parameter $\gamma$ indicates at which level of savings the change takes place. We take the savings level 2 periods back to enable us to consider it predetermined. Recently developed techniques for testing for the existence and estimating the threshold levels are then used.

\footnotetext{
${ }^{4}$ For further details on the problem of identification, see Latkepohl (1991).
} 


\subsection{Econometric considerations}

The econometric analysis of model (5.4), a vector autoregressive model with pooled coefficients, is similar to that of a large VAR with restrictions on the coefficients of the lag polynomial. Due to the fact that we assume $T$ to go to infinity we can apply the standard asymptotic theory on stationary vector autoregressions ${ }^{5}$, taking into account the restrictions.

The analysis of the threshold model (5.5) is not straightforward. There are several problems which complicate the analysis. First, under the null hypothesis of no difference between the $B_{0}=\bar{B}_{0}$ and $B_{1}=\bar{B}_{1}$ the threshold parameter $\gamma$ is not identified. This complicates the analysis considerably but recent econometric research such as Hansen (1995), (1996), Andrews, Lee and Ploberger (1996), Andrews and Ploberger (1994), provides the tools to tackle this problem. Some care, however, is necessary when adopting their framework to our specific case. For one, most of the above chapters deal with time series and/or cross section data while our approach is based on the analysis of a panel data set. Furthermore, some of the procedures suggested in the literature are tedious to calculate as they rely on bootstrapping methods while others involve the choice of some, rather subjectively chosen, parameters. In contrast, the simple test presented in section 5.3.1 avoids both problems.

Another not straightforward problem is to perform the impulse response analysis in the threshold model. This model is not linear and therefore many of the nice properties of the impulse response function in the linear model do not hold. The only practical way to deal with this problem is using simulation/bootstrapping. For an introduction into impulse response analysis of threshold or other nonlinear dynamic models we refer to Potter (1995) and Gallant, Rossi and Tauchen (1993). In section 5.3.2 we will discuss some of the important points which are important in the discussion of our empirical results.

\subsubsection{Testing for the existence of a threshold}

To introduce our analysis of threshold models we choose the following setup: Assume that we have observed the variables $\left(Y_{i}, X_{i}\right)$ for $i=1, \ldots, N$ with $X_{i}$ a $\left(1 \times k_{x}\right)$ vector and $Y_{i}$ a scalar variable. Let the following relation hold:

$$
Y_{i}=X_{i} \beta_{1}+Z_{i} I\left(q_{i}>\gamma\right) \beta_{2}+\varepsilon_{i}
$$

where $Z_{i}$ a $\left(1 \times k_{z}\right)$ vector, $q_{i}$ a one dimensional variable and $\gamma$ a scalar which we assume to be contained in a compact subset $\Gamma$ of $R$. We assume that the variables in $Z_{i}$ are also contained in $X_{i}$, i.e. $Z_{i}$ is a subvector of $X_{i}$. Finally we assume $\varepsilon_{i}$ to be a zero mean i.i.d distributed random variable with finite variance $E\left(\varepsilon_{i}\right)^{2}=\sigma^{2}$. Observe that the indicator function $I(\bullet)$ equals one when the condition within the brackets is satisfied and zero otherwise. In this way it determines a break between observations satisfying this condition and observations which do not satisfy this condition.

We analyze this model in a time series context, that is we allow for lagged observations in the $X_{i}, Z_{i}$, and $q_{i}$. The null hypothesis we are interested in is $H_{0}: \beta_{2}=0$ and the

\footnotetext{
${ }^{5}$ See, for example, Hamilton (1994) and Lutkepohl (1991) for an introduction to this analysis.
} 
alternative hypothesis $H_{1}: \beta_{2} \neq 0$. Under the null this means that the parameter $\gamma$ is not identified. For fixed known $\gamma$ classical hypothesis testing theory can be used. However in this case, where $\gamma$ is unknown, we cannot apply the standard tests. A number of researchers have addressed this problem and developed appropriate tests. We mention explicitly the supremum (sup), the average (ave) and exponential (exp) versions of the Wald, LM, and LR statistics introduced in Andrews, Lee and Ploberger (1996), Andrews and Ploberger (1994) and Hansen (1995), (1996).

Our test procedure, which is related to these tests, can be derived in two ways. The first possibility is to consider the LM version, i.e make only use of the restricted estimator obtained under the restriction $\beta_{2}=0$. Under $H_{0}$, we obtain using OLS the following, restricted, estimator

$$
\beta_{1}^{\mathrm{r}}=\left(X^{\prime} X\right)^{-1} X^{\prime} Y
$$

where $X=\left(X_{1}^{\prime}, \ldots, X_{N}^{\prime}\right)^{\prime}$ and $Y=\left(Y_{1}^{\prime}, \ldots, Y_{N}^{\prime}\right)^{\prime}$. Denote the residuals of the restricted estimator by $u^{r}=Y-X \hat{\beta_{1}^{r}}$. Further we introduce the function $Z_{(\gamma)}=\left(Z_{1}^{\prime} I\left(q_{1}>\right.\right.$ $\left.\gamma), \ldots, Z_{N}^{\prime} I\left(q_{N}>\gamma\right)\right)^{\prime}$ and observe

$$
\begin{aligned}
Z_{(\gamma)}^{\prime} u^{r} & =Z_{(\gamma)}^{\prime}\left(Y-X \hat{\beta}_{1}^{r}\right)=Z_{(\gamma)}^{\prime} M Y \\
& =Z_{(\gamma)}^{\prime} M\left(X \beta_{1}+Z_{(\gamma)} \beta_{2}+\varepsilon\right) \\
& =Z_{(\gamma)}^{\prime} M\left(Z_{(\gamma)} \beta_{2}+\varepsilon\right)
\end{aligned}
$$

with $M$ being the projection matrix $M=\left(I-X\left(X^{\prime} X\right)^{-1} X^{\prime}\right)$ onto subspace spanned by $X$. We have

$$
\begin{array}{ll}
Z_{(\gamma)}^{\prime} u^{r}=Z_{(\gamma)}^{\prime} M \varepsilon & \text { under } H_{0}, \\
Z_{(\gamma)}^{\prime} u^{r}=Z_{(\gamma)}^{\prime} M Z_{(\gamma)} \beta_{2}+Z_{(\gamma)}^{\prime} M \varepsilon & \text { under } H_{1} .
\end{array}
$$

In the appendix we show that the normalized stochastic function

$$
z_{N}(\gamma)=\frac{1}{\sqrt{N}} Z_{(\gamma)}^{\prime} u^{r}
$$

converges to a $k_{z}$ dimensional Gaussian process as $N \rightarrow \infty$ with covariance kernel $\lim _{N \rightarrow \infty} \sigma_{N}\left(\gamma_{1}, \gamma_{2}\right)$ where

$$
\sigma_{N}\left(\gamma_{1}, \gamma_{2}\right)=\frac{1}{N} \sigma^{2} Z_{\left(\gamma_{1}\right)}^{\prime} M Z_{\left(\gamma_{2}\right)}
$$

As it is difficult to test whether the stochastic function (5.13) satisfies the behavior associated with the null hypothesis we use a transformation to summarize its behavior in one test statistic. We introduce the integrals

$$
\int_{\Gamma} \mathcal{S}\left(z_{N}(\gamma) z_{N}^{\prime}(\gamma)\right) d \gamma, \text { and } \int_{\Gamma} \mathcal{S}\left(\sigma_{N}(\gamma, \gamma)\right) d \gamma
$$

where $\mathcal{S}(\bullet)$ denotes the operator defined as the sum of all elements of its argument. Finally the test statistic is defined as

$$
W=\int_{\Gamma} \mathcal{S}\left(z_{N}(\gamma) z_{N}^{\prime}(\gamma)\right) d \gamma / \int_{\Gamma} \mathcal{S}\left(\sigma_{N}(\gamma, \gamma)\right) d \gamma
$$


Under the assumptions given in the appendix, that is the same assumptions as in Hansen (1996), we can derive an approximation of the asymptotic distribution of $W$, see the discussion in Appendix C. The following critical regions are given in Bierens and Ploberger (1995)

$$
P(W>3.23)=0.10, \quad P(W>4.26)=0.05, \quad P(W>6.81)=0.01 .
$$

A much more detailed discussion is given in the appendix. The test is simple because we only have to calculate the integrals in (5.14). A disadvantage of our procedure is that it can be conservative. The degree of conservatism will depend on the problem at hand. In the appendix we show the connection between our and some LM statistics proposed in the literature. It turns out that our test is less powerful then the $\sup L M$, aveLM and $\exp L M$ as proposed by Andrew (1993) and Andrews and Ploberger (1996). However our test is very convenient to use as the critical values are given above and they are independent of other parameters such as for instance the number of regressors. In addition we have some more detailed information about the power of the test. A result concerning a family of local alternatives is given in the appendix.

\subsubsection{Inference on the threshold}

Once we have concluded, using the tests above, that there is a threshold we also need an estimate threshold parameter $\gamma$ and the corresponding estimates for $\beta_{1}$ and $\beta_{2}$. We solve this problem as follows. The estimator for $\gamma$ and the other parameters is given by the solution of the nonlinear least squares problem:

$$
\left(\hat{\gamma}, \hat{\beta}_{1}, \hat{\beta_{2}}\right)=\arg \min _{\gamma \in \Gamma_{,}, \beta_{1}, \beta_{2}}\left(Y-X \beta_{1}-Z_{(\gamma)} \beta_{2}\right)^{\prime}\left(Y-X \beta_{1}-Z_{(\gamma)} \beta_{2}\right)
$$

Finding the global minimum can be done in two steps; first we minimize the sum of squared errors for fixed $\gamma$. This minimum is found by applying OLS and gives an estimate of the variance of the residuals $\hat{\sigma}^{2}(\gamma)$. Second we minimize $\hat{\sigma}^{2}(\gamma)$ over all $\gamma \in \Gamma$. The final estimates are then the OLS coefficients corresponding to that $\gamma$ which minimized $\hat{\sigma}^{2}(\gamma)$. Observe that when the errors $\epsilon_{i}$ are i.i.d $N\left(0, \sigma^{2}\right)$ this estimator is also the MLE.

As is known from the literature, see Bai (1995), Picard (1985), Chan (1993) this estimator for $\gamma$ has a convergence rate of order $n$, which is much faster than the order of convergence $(\sqrt{n})$ for the other parameters in the model. The derivation of the asymptotic distribution of the estimator for $\gamma$ has proved to be a rather hard problem. When the difference between $\beta_{1}, \beta_{2}$ is considered to be fixed, or relatively large, the distance between the two appears in the asymptotic distribution of $\hat{\gamma}$ which makes inference almost intractable. However, under the assumption of a local alternative, i.e. a small difference -depending on the sample size- between $\beta_{1}, \beta_{2}$, Hansen (1996) derives the asymptotic distribution of the Likelihood Ratio statistic for the test $\gamma=\gamma_{0}$ and uses this result to construct a confidence interval for $\gamma$. We will present confidence bounds based on this procedure in the empirical results. 


\subsubsection{Nonlinear impulse responses}

Computing impulse response functions for nonlinear dynamic models is more complicated than computing impulse response functions for linear dynamic models for several reasons. One of the complications is that in most cases there are no analytical results as is in the linear case. This means that the impulse responses must be obtained numerically or simulated. Further it is much harder to present and investigate all the information contained in the impulse response of a nonlinear system. This is due to the fact that the responise of a nonlinear system to a shock at time $t_{0}$ is path-dependent. The response depends in a nonlinear way on the history of the system, i.e., the observations before the shock enters the system and on the disturbances which come into the system between time $t_{0}$ and $t_{0}+k$. Finally, the proportionality of a response to the size of a shock in a linear system does not hold in a nonlinear system. More details on the problems of analyzing nonlinear impulse response can be found, amongst others, in Gallant, Rossi and Tauchen (1993) and Potter (1995).

In our analysis we use the following tools: For the linear VAR's we give the traditional impulse responses for a linear VAR, including \pm 2 standard-errors confidence bounds based on 500 drawings from the distribution of the estimates of the parameters.

For the nonlinear threshold VAR we estimate the following type of impulse response function, corresponding to the Generalized Impulse Response function as proposed by Potter (1995)

$$
G I\left(t_{0}, k, \delta, \Omega_{t_{0}-1}\right)=E\left(X_{t_{0}+k} \mid \Omega_{t_{0}-1}, \varepsilon_{t_{0}}=\delta\right)-E\left(X_{t_{0}+k} \mid \Omega_{t_{0}-1}\right),
$$

where $\Omega_{t_{0}-1}$ is the history at time $t_{0}$, and $\delta$ is the shock given to the system at time $t_{0}$. This impulse response function was for instance also analyzed in for instance Balke and Chang (1995). To obtain this impulse response function we need to integrate out the future shocks $\varepsilon_{t_{0+1}}, \ldots, \varepsilon_{t_{0+k}}$, in fact

$$
\begin{array}{r}
G I\left(t_{0}, k, \delta, \Omega_{t_{0-1}}\right)=E_{\varepsilon_{t_{0+1}} \ldots, \varepsilon_{t_{0+k}}}\left(E\left(X_{t_{0}+k} \mid \Omega_{t_{0}-1}, \varepsilon_{t_{0}}=\delta_{y} \varepsilon_{t_{0+1}}, \ldots, \varepsilon_{t_{0+k}}\right)\right. \\
\left.-E\left(X_{t_{0}+k} \mid \Omega_{t_{0}-1}, \varepsilon_{t_{0+1}}, \ldots, \varepsilon_{t_{0+k}}\right)\right) .
\end{array}
$$

To do this we generate a large number of future i.i.d zero mean normal shocks $\varepsilon_{t_{0+1}}^{i}, \ldots, \varepsilon_{t_{0+k}}^{i}$, $i=1, \ldots, R$, and estimate $G I\left(t_{0}, k, \delta, \Omega_{\mathrm{t}_{0}-1}\right)$ by calculating

$$
\begin{aligned}
& \stackrel{\wedge}{G I}\left(t_{0}, k, \delta, \Omega_{t_{0}-1}\right) \\
= & \frac{1}{R} \sum_{i=1}^{R} E\left(X_{t_{0}+k} \mid \Omega_{t_{0}-1,}, \varepsilon_{t_{0}}=\delta, \varepsilon_{t_{0+1}}^{i}, \ldots, \varepsilon_{t_{0+k}}^{i}\right) \\
& -\frac{1}{R} \sum_{i=1}^{R}\left(E X_{t_{0}+k} \mid \Omega_{t_{0}-1}, \varepsilon_{t_{0+1}}^{i}, \ldots, \varepsilon_{t_{0+k}}^{i}\right),
\end{aligned}
$$

i.e. we average the impulse response function over the future shocks.

We further notice that $G I\left(t_{0}, k, \delta, \Omega_{t_{0}-1}\right)$ depends on $\Omega_{t_{0}-1}$. In a threshold model for instance it makes a big difference whether the system is close to the threshold level at $t_{0}$, the time of the shock, or not. Therefore we consider $G I\left(t_{0}, k, \delta, \Omega_{t_{0}-1}\right)$ conditional an 
different histories $\Omega_{t_{0}-1}$. Note that we estimate the parameters of the system using all information available.

In our case we are especially interested in the behavior of the system if we know that it is in the high or the low regime. Therefore we also calculate $G I\left(t_{0}, k, \delta, \Omega_{t_{0}-1}\right)$ for the following three situations. We present impulse responses 1) unconditional on the regime we are at time $t_{0}, 2$ ) conditional on being in the high savings regime at time $t_{0}$ and 3 ) conditional on being in the low savings regime at time $t_{0}$. We generated 100 histories, simulating the model and leaving out the first 500 observations to avoid initial condition problems, satisfying the three different conditions and for each history we calculated the generalized impulse response function based on 100 sets of future shocks $(R=100)$.

Finally, we also take into account the fact that we estimated the parameters of the system. We draw 50 sets of parameters from the asymptotic distribution of the estimated parameters. For each set of parameters we replicated the above procedure. So for instance, we calculated the impulse response (5.16) conditional on being in the high regime $50 * 100=5000$ times.

As the impulse response function of a threshold model can be asymmetric we investigated shocks of size -1 and +1 . We present the average and the $95 \%$ most centered realizations of (5.16) for each of the three classes of histories. Finally we present the average of (5.16) for each of the three classes of histories with its two $95 \%$ confidence bounds.

\subsection{The data}

Per capita GDP, saving rate and openness are taken from World Bank data (1996). The saving rate is based on gross domestic savings which equals GDP minus consumption, and hence is the sum of private (household and business) and government savings. The saving rate is the ratio of nominal gross domestic savings to nominal GDP (both in local currency), while openness to trade is the ratio of nominal exports plus imports to nominal GDP (both again in local currency). These data are generally available for most countries for the period between 1960 and 1992. Excluding oil exporting countries as well as countries with a population of less than one million, 58 countries remain in the data set. The sample period covers the years from 1960 to 1987. The data for 1960 and 1961 are used as initial values.

The GDP growth is the log difference of per capita GDP in constant 1987 value of the local currency). Openness is transformed into logs, the savings ratio however is not transformed into logs because a small percentage of the data takes on negative values, The scale of the saving rate is of the same order as GDP growth and openness, so that the scale does not form a problem. In the Figure 5.1 we give the empirical distributions of the collected data. As one can see the saving rate displays a good deal of variability. Observe that this variability is necessary to be able to verify our hypothesis of a changing relation between GDP growth and openness. 

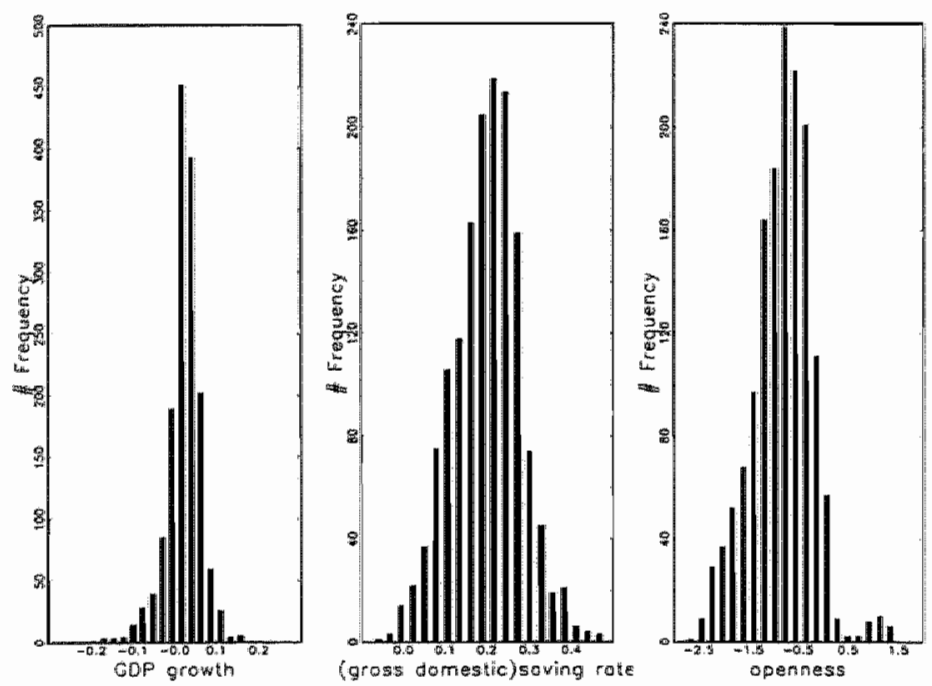

Figure 5.1: Distribution of the data.

\subsection{Empirical Results}

\subsubsection{Long-run effects}

We first consider the long-run relation between the savings ratio and GDP growth which is crucial in most theories on endogenous growth. Applying OLS to time averaged variables for each country, i.e. $\Delta Y_{i}=\frac{1}{26} \sum_{t} \Delta Y_{i, t}, S_{i_{1}-1}=\frac{1}{26} \sum_{t} S_{i, t-1}$ gives the following result:

$$
\Delta Y_{i}=\underset{(0.006)}{0.007}+\begin{gathered}
0.083 S_{i,-1} \\
(0.029)
\end{gathered} \quad R^{2}=0.13, \quad N=58
$$

Using the White test, see White (1980), for heteroscedasticity: $n R^{2}=1.972$, which under the null of heteroscedasticity has a chi-squared distribution with 3 degrees of freedom, we cannot reject homoscedasticity. We further tested for normality of the residuals but we could not reject normality.

The estimated coefficient for savings is positive and significant at the $5 \%$ level. Note that this result is based on the assumption of parameter equality across countries. Adding (lagged) openness to the above estimation model yields the following result:

$$
\Delta Y_{i}=\underset{(0.007)}{0.012}+\underset{0.083 S_{i_{1}-1}}{(0.029)}+\underset{0.005 O_{i,-1}}{(0.003)} \quad R^{2}=0.16, \quad N=58
$$

Again we can not reject homoscedasticity using White's test: $n R^{2}=2.826$, which under the null has a chi-squared distribution with 6 degrees of freedom. We also cannot reject normality of the residuals. 


\begin{tabular}{|c|c|c|}
\hline Type of Test & Value test statistic & $\mathrm{p}$-value \\
\hline$B P H$ & 5.44 & $0.05<p<0.01$ \\
\hline $\exp L M$ & 3.92 & $0.1<p<0.05$ \\
\hline ave $L M$ & 7.05 & $0.05<p<0.01$ \\
\hline sup $L M$ & 10.23 & $>0.10$ \\
\hline Hansen $F$ & 13.98 & 0.074 \\
\hline & & \\
\hline & Estimate of threshold & $95 \%$ conf. interval. \\
\hline & 0.1442 & $(0.049,0.248)$ \\
\hline
\end{tabular}

Table 5.1: Tests for the existence of a threshold.

Clearly, the effect of openness is small and insignificant as is found by Harrison. (1996). Next we test for the existence of a threshold. We perform three LM tests ( aveLM, $\operatorname{expLM,}$ supLM), the F-test proposed by Hansen (1997), denoted by HansenF, and our own test given in (5.15), denoted by BPH (Bierens-Ploberger-Hoogstrate). Since the regressors are averages over time the threshold at a certain time would be conditioned on future values of the regressors which would lead to inconsistent estimates. To avoid this problem we use the savings ratio of 1960 as threshold variable. The test for a threshold is given in Table 5.1. We conducted the LM tests with $\pi_{0}=0.15$, (see for the definition of $\pi_{0}$ Andrews and Ploberger (1994) or Andrews (1993)). We searched for a threshold among countries with a. savings ratio in the interval $(0.095,0.276)$. As we see in four out of five cases the null of no threshold is rejected. The resulting estimate for the threshold is 0.1442 . Substituting this value for $\gamma$ we obtain for the low savings countries $S_{i,-2} \leq \gamma=0.144$

$$
\Delta Y_{i}=\underset{(0.011)}{0.001}+\underset{(0.058)}{0.186 S_{i,-1}}+\underset{(0.005)}{0.005 O_{i,-1}} \quad R^{2}=0.38, \quad N=22
$$

and for the high savings countries, $S_{i,-2}>\gamma=0.144$

$$
\Delta Y_{i}=\begin{gathered}
-0.003 \\
(0.011)
\end{gathered}+\begin{gathered}
0.107 S_{i,-1} \\
(0.040)
\end{gathered}-\begin{gathered}
0.005 O_{i,-1} \\
(0.004)
\end{gathered} R^{2}=0.22, \quad N=36
$$

with a joined $R^{2}=0.32$. Interestingly, the sign on the coefficient of openness varies between the two subgroups of countries but in both cases the parameters are insignificant. Also compared to the joint estimation, the estimated coefficient for the average saving rate is larger for both subgroups. Observe the change in the parameter on the average savings ratio.

We compare the above results to the results of the dynamic analysis below later.

\subsubsection{VAR analysis: estimation and testing}

First we investigate whether any of the variables has a unit root. For this we use the test statistics developed by Im et al. (1996). They develop unit root tests for heterogeneous panels specified by

$$
x_{i, t}=\left(1-\phi_{i}\right) \mu_{i}+\phi_{i} x_{i, t-1}+\varepsilon_{i, t}
$$




\begin{tabular}{|l|l|l|}
\hline Variable & $\mathbf{L R}_{26}(0,0)$ & p-vallue \\
\hline GDP growth & 2.85 & 0.002 \\
saving rate & 3.164 & $<0.001$ \\
openness & -0.884 & 0.199 \\
\hline
\end{tabular}

Table 5.2: Tests for unit roots.

where $E\left(\varepsilon_{i, t}\right)=\sigma_{i}^{2}$. They test the hypothesis $\phi_{i}=1$ for all $i$ against $\phi_{i}<1$ for all $i$. Observe that our model is contained in this model. We therefore can use this procedure to test for unit roots, while being robust against possible misspecification due to the imposed restrictions, $\phi_{i}=\phi$ and $\mu_{i}=\mu$ for all $i$, in our model. The results are given in Table 5.2. This result indicates that we can formally reject the hypothesis of a unit root in the GDP growth and in the saving rate series. For the openness series however we cannot reject the hypothesis of a unit root. This result has some implications for the model we proposed in (5.4) and (5.5).

As the series $O_{i, t}$ is $I(1)$ and the series $\Delta y_{i, t}$ and $S_{i, t}$ are $I(0)$ we have to put in restrictions on the coefficients of openness to obtain a stationary system. Therefore we restrict the coefficients of current and lagged openness to be equal but of opposite sign in all equations, also in the threshold model given by (5.5). This means that we can restate (5.4) and (5.5) in terms of $\Delta O_{i, t}=O_{i, t}-O_{i, t-1}$

$$
X_{i, t}^{*}=\mu+B_{0} X_{i, t}^{*}+B_{1} X_{i, t-1}^{*}+u_{i t}
$$

and

$$
\begin{aligned}
X_{i, t}^{*}= & \left(\mu+B_{0} X_{i, t}^{*}+B_{1} X_{i, t-1}^{*}\right) I\left(S_{i, t-2} \leq \gamma\right) \\
& +\left(\bar{\mu}+\bar{B}_{0} X_{i, t}^{*}+\bar{B}_{1} X_{i, t-1}^{*}\right) I\left(S_{i, t-2}>\gamma\right)+w_{i t}
\end{aligned}
$$

where $X_{i, t}^{*}=\left(\Delta O_{i t}, S_{i, t}, \Delta Y_{i t}\right)^{\prime}$ with the following zero restrictions:

$$
B_{0}, B_{0}, \bar{B}_{0}:\left[\begin{array}{ccc}
0 & 0 & 0 \\
& 0 & 0 \\
& & 0
\end{array}\right] \text {, and } B_{1}, B_{1}, \bar{B}_{1}:\left[\begin{array}{l}
0 \\
0 \\
0
\end{array}\right] \text {. }
$$

In Table 5.3 we present the estimation results of (5.21) under the assumption of homoscedasticity. We assume that $\Lambda_{i}$, the matrix with the variances of disturbances, is the same for each country. As explained above we can estimate this system as three separate equations with GDP growth, the saving rate and change in openness as the respective dependent variables (standard errors are given in parenthesis).

In the first regression (with GDP growth as the dependent variable) present and past saving rates have a significant impact on GDP growth, while the change in openness is insignificant. This finding for openness is similar to the results in previous empirical studies which also find that openness has no significant impact on GDP growth (see, for example, Harrison (1996)). As the next column in Table 5.3 reveals, however, the result does not imply that openness has no effect on GDP growth. In the second equation (with savings as the dependent variable) a change in openness has a significant impact on the saving rate which, in turn, has a significant relation with GDP growth. 


\begin{tabular}{|c|c|c|c|}
\hline & \multicolumn{3}{|c|}{ dependent variables } \\
\hline regressors & $\Delta y_{i, t}$ & $\overline{S_{i, t}}$ & $\Delta O_{i, t}$ \\
\hline$c$ & $\begin{array}{l}0.004 \\
(0.003)\end{array}$ & $\begin{array}{l}0.016 \\
(0.002)\end{array}$ & $\begin{array}{l}0.011 \\
(0.008)\end{array}$ \\
\hline$S_{i, t}$ & $\begin{array}{l}0.344 \\
(0.032)\end{array}$ & - & - \\
\hline$\Delta O_{i, t}$ & $\begin{array}{l}0.000 \\
(0.008)\end{array}$ & $\begin{array}{l}0.012 \\
(0.006) \\
\end{array}$ & - \\
\hline$\Delta y_{i, t-1}$ & $\begin{array}{l}0.265 \\
(0.024)\end{array}$ & $\begin{array}{l}0.047 \\
(0.019)\end{array}$ & $\begin{array}{l}0.083 \\
(0.076)\end{array}$ \\
\hline$S_{i, t-1}$ & $\begin{array}{l}-0.279 \\
(0.032)\end{array}$ & $\begin{array}{l}0.919 \\
(0.010) \\
\end{array}$ & $\begin{array}{l}-0.017 \\
(0.039)\end{array}$ \\
\hline$R^{2}$ & 0.16 & 0.86 & 0.001 \\
\hline
\end{tabular}

Table 5.3: Estimation results linear VAR under homoscedasticity.

The third regression (with the change of openness as dependent variable) shows that the lagged GDP growth and saving rate have no significant impact on the change of openness. This means that in this case we might consider the bivariate model for the saving rate and GDP growth with the change in openness as exogenous variable. As we will see in later this does not hold for some of the other models. We therefore proceed with the analysis as if the change in openness is endogenous. The complexitiy of the relation between openness and growth will become clearer when we present the results of the impulse response analysis in the next section.

Next, we test for the existence of a threshold. As before, we perform three LM tests (ave $L M$, expLM, supLM), the F-test proposed by Hansen (1997), denoted by HansenF, and our own test given in (5.15), denoted by BPH. In a VAR system as the one we analyze there are two ways to model the breakpoint. A threshold can be modeled separately for each of the three equations. Not surprisingly, we may find a different threshold for each equation in this case. Alternatively, we can model one threshold level restricted to be the

\begin{tabular}{|c|c|c|c|c|}
\hline & 1. equation & 2. equation & 3. equation & Restricted \\
\hline Test & $\Delta y_{i, t}$ & $S_{i, t}$ & $\Delta O_{i, t}$ & $V A R$ \\
\hline$B P H$ & 1.07 & $9.68^{* *}$ & 3.51 & 2.91 \\
\hline expLM & $12.80^{* *}$ & $19.48^{* *}$ & $8.81^{* * *}$ & $37.73^{* *}$ \\
\hline aveLM & $13.76^{* *}$ & $24.83^{* *}$ & $12.41^{* *}$ & $51.33^{* *}$ \\
\hline supLM & $32.66^{* *}$ & $44.25^{* *}$ & $23.83^{* *}$ & $84.98^{* *}$ \\
\hline Hansen $F$ & $38.80^{* *}$ & $46.42^{* *}$ & $32.66^{* *}$ & $88.69^{* *}$ \\
\hline & & & & \\
\hline$\gamma$ & 0.249 & 0.196 & 0.162 & 0.249 \\
\hline $95 \%$ conf. inter. & $(0.248,0.277)$ & $(0.177,0.249)$ & $(0.044,0.193)$ & $(0.193,0.253)$ \\
\hline *indicates & $5 \%$ significance & & **indicates $^{* *}$ & $1 \%$ significance \\
\hline
\end{tabular}

Table 5.4: Testing for the existence of a threshold in the VAR under homoscedasticity. 


\begin{tabular}{|c|c|c|c|c|c|c|}
\hline & \multicolumn{3}{|c|}{$S_{i, t-2}<=0.249, N=1108$} & \multicolumn{3}{c|}{$S_{i, t-2}>0.249, N=400$} \\
\hline & \multicolumn{5}{|c|}{ dependent variables } \\
\hline regressors & $\Delta y_{i, t}$ & $S_{i, t}$ & $\Delta O_{i, t}$ & $\Delta y_{i, t}$ & $S_{i, t}$ & $\Delta O_{i, t}$ \\
\hline \multirow{2}{*}{$c$} & 0.004 & 0.017 & -0.016 & 0.010 & 0.014 & 0.015 \\
& $(0.003)$ & $(0.003)$ & $(0.010)$ & $(0.011)$ & $(0.008)$ & $(0.025)$ \\
\hline \multirow{2}{*}{$S_{i, t}$} & 0.307 & - & -- & 0.534 & -- & -- \\
& $(0.036)$ & - & $(0.072)$ & & - \\
\hline \multirow{2}{*}{$\Delta O_{i, t}$} & 0.007 & -0.002 & -- & -0.049 & 0.109 & - \\
& $(0.009)$ & $(0.007)$ & & $(0.023)$ & $(0.015)$ & - \\
\hline \multirow{2}{*}{$\Delta y_{i, t-1}$} & 0.345 & 0.046 & 0.042 & 0.079 & 0.023 & 0.034 \\
& $(0.028)$ & $(0.023)$ & $(0.097)$ & $(0.045)$ & $(0.031)$ & $(0.103)$ \\
\hline \multirow{2}{*}{$S_{i, t-1}$} & -0.252 & 0.905 & 0.094 & -0.469 & 0.931 & -0.086 \\
& $(0.037)$ & $(0.014)$ & $(0.060)$ & $(0.077)$ & $(0.026)$ & $(0.087)$ \\
\hline & $R^{2}=0.19$ & $R^{2}=0.79$ & $R^{2}=0.002$ & $R^{2}=0.13$ & $R^{2}=0.78$ & $R^{2}=0.027$ \\
\hline
\end{tabular}

Table 5.5: Estimation results for the threshold model under homoscedasticity.

same for all three equations. To get an idea of the effects we pursue both approaches with the results presented in Table 5.4 below together with the results for the five different test statistics. To avoid technical problems we use $S_{i, t-2}$ as threshold variable.

The aveLM, $\exp L M$ and supLM tests are based on $\pi=0.05$. The F-test of Hansen is derived under the assumption of maintained homogeneity of the variances of the disturbances among the two groups. First notice that, as expected (see the appendix C) the $\mathrm{BPH}$ test suffers from a lack of power as compared to the other tests. As expected, the threshold estimates differ across the three equations. As we do not have a reasonable economic interpretation for different thresholds for the different variables we further restrict our attention to the case where the thresholds are the same for every equation.

While the regression results for the single equations are given in appendix $A$, we present the results for the VAR with the restricted threshold level in Table 5.5 (standard errors are in parenthesis).

There are 1108 observations in the low savings regime and 400 observations in the high savings regime. Furthermore, while 20 countries are in the low saving regime at every point in time, only 2 countries are always in the high saving regime. All other countries switch regimes at some point(s) during the sample period.

The above results, except the test for the unit root are based on the assumption of equal variances for the errors across countries. If we however perform a Bartlett test for the presence of heteroscedasticity the null of homoscedasticity is rejected very strongly for each of the equations. Therefore we extended our analysis by weakening the assumption of homoscedasticity by assuming (5.4) to hold with $\Lambda_{i}=\operatorname{diag}\left(\sigma_{i, 1}^{2}, \sigma_{i, 2}^{2}, \sigma_{i, 3}^{2}\right)$. The estimated model is given in Table 5.6. Notice that the fit has improved.

To check the validity of this model further we performed the Roy-Zellner test for poolability. For the GDP growth and the saving rate regression we obtain the F-values 3.134 ( $\mathrm{p}$-value $<0.001$ ) and 2.426 (p-value $<0.001$ ) respectively, which means that we reject the null hypothesis of all coefficients being the same for each country for these regressions. This implies that the linear model is misspecified, but in our case this is just 


\begin{tabular}{|c|c|c|c|}
\hline & \multicolumn{3}{|c|}{ dependent variables } \\
\hline regressors & $\Delta y_{i, t}$ & $\overline{S_{i, t}}$ & $\Delta O_{i, t}$ \\
\hline$c$ & $\begin{array}{l}0.005 \\
(0.002)\end{array}$ & $\begin{array}{l}0.011 \\
(0.002)\end{array}$ & $\begin{array}{l}0.008 \\
(0.007)\end{array}$ \\
\hline$S_{i, t}$ & $\begin{array}{l}0.522 \\
(0.031)\end{array}$ & - & - \\
\hline$\Delta O_{i, t}$ & $\begin{array}{l}0.033 \\
(0.007)\end{array}$ & $\begin{array}{l}0.014 \\
(0.005)\end{array}$ & - \\
\hline$\Delta y_{i, t-1}$ & $\begin{array}{l}0.302 \\
(0.022)\end{array}$ & $\begin{array}{l}0.066 \\
(0.016)\end{array}$ & $\begin{array}{l}0.181 \\
(0.062)\end{array}$ \\
\hline$S_{i, t-1}$ & $\begin{array}{c}-0.466 \\
(0.031)\end{array}$ & $\begin{array}{l}0.942 \\
(0.007)\end{array}$ & $\begin{array}{l}0.011 \\
(0.032)\end{array}$ \\
\hline$R^{2}$ & 0.41 & 0.98 & 0.009 \\
\hline
\end{tabular}

Table 5.6: Estimation results for the linear VAR under heteroscedasticity.

what we expect: If we had to accept this pooling restriction a threshold could not exist; It would not be possible to have countries in different regimes. We use the pooled models without threshold as a reference, not as the models to derive conclusions. For the change in openness however we do not reject the null hypothesis of pooling. The $F$ value equals 1.0048 with associated p-value 0.4717 .

In Table 5.7 we present the results of the tests for the existence of a threshold. Comparing Table 5.4 and Table 5.7 we see that the results have changed considerably. Especially the results for the VAR model writh the threshold restricted to be the same for each variable. The estimated thresholds changed from 0.249 in the homoscedastic model to 0.192 in the heteroscedastic model. Observe that this seems to indicate that the threshold has the largest effect on the GDP regression. The coefficients for the resulting threshold VAR are given in the Table 5.8. There are now 670 observations in the low savings regime and 838 observations in the high savings regime.

Observe that also in this case the fit has improved considerably. Notice further that the

\begin{tabular}{|c|c|c|c|c|}
\hline & 1. equation & 2. equation & 3. equation & Restricted \\
\hline Test & $\Delta y_{i, t}$ & $S_{i, t}$ & $O_{i, t}$ & $V A R$ \\
\hline$B P H$ & 1.62 & 2.40 & $3.23^{*}$ & 1.85 \\
\hline $\exp L M$ & $17.80^{* *}$ & $10.74^{* *}$ & $7.41^{*}$ & $34.50^{* *}$ \\
\hline ave $L M$ & $15.78^{* *}$ & $12.29^{* *}$ & $11.25^{* *}$ & $40.00^{* *}$ \\
\hline supLM & $41.92^{* *}$ & $27.66^{*}$ & $19.51^{*}$ & $77.66^{* *}$ \\
\hline HansenF & $45.15^{* *}$ & $30.73^{* *}$ & $21.02^{*}$ & $49.82^{* *}$ \\
\hline & & & & \\
\hline$\gamma$ & 0.181 & 0.240 & 0.154 & 0.192 \\
\hline $95 \%$ conf. inter. & $(0.177,0.204)$ & $(0.229,0.249)$ & $(0.075,0.227)$ & $(0.180,0.247)$ \\
\hline *indicates & $5 \%$ significance & & **indicates & $1 \%$ significance \\
\hline
\end{tabular}

Table 5.7: Testing for the existence of threshold in the VAR under heteroscedasticity. 


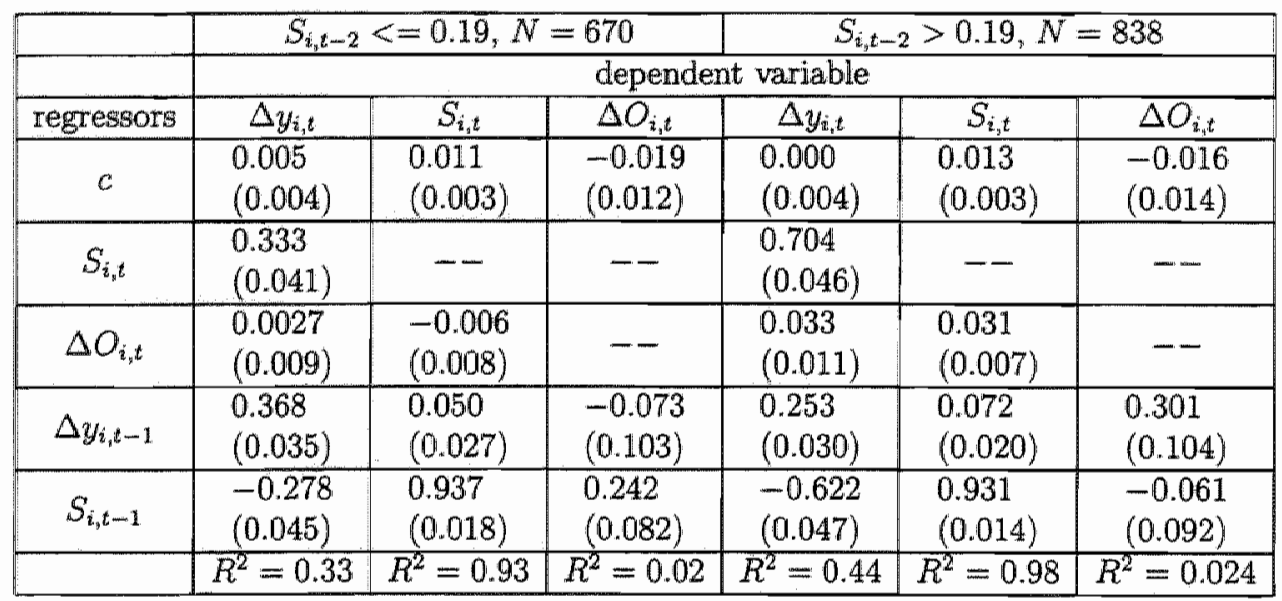

Table 5.8: Estimation results for the threshold model under heteroscedasticity.

change in openness is significantly affected by the lagged saving rate in the low regime and lagged GDP growth in the high regime.

\subsubsection{VAR analysis: Impulse responses}

In this section we discuss the impulse response analysis. The impulse response analysis is based on the models as identified by the Tables 5.3 and 5.6 for the linear models and Tables 5.5 and 5.8 for the nonlinear threshold models.

Observe that tests in the previous section test for the existence of a threshold for all the parameters simultaneously, i.e. when only one of the coefficients of $\mu, B_{0}$ or $B_{1}$ changes significantly between regimes the test will detect this. This does not mean that all coefficients differ across the regimes. However in our following analysis we will use the estimates based on the assumption that all coefficients change across regimes. This assumption will not restrict the validity of our analysis or possible outcomes, but we only lose some efficiency by not imposing possible restrictions

We now start with the impulse response analysis. The impulse responses from the linear VAR without threshold with the parameters as given in Table 5.3 are presented in Figure 5.2. We use unit shocks. Further note that in our model the innovations are already orthogonal.

The impulse responses for the heteroscedastic linear VAR from Table 5.6 are given in Figure 5.3. A first striking difference between the two systems is that in the system resulting from the homoscedastic assumption there is no significant response from the saving rate or GDP growth to a shock in openness, this in contrast to the system resulting from the heteroscedastic assumption where there are significant responses of GDP growth and savings to a change in openness. A second observation is that a shock in the saving rate or GDP growth has a significant effect on openness in the system resulting from the heteroscedastic assumption. This is in contrast to the insignificant impact of these shocks in the system with the homoscedastic errors. 

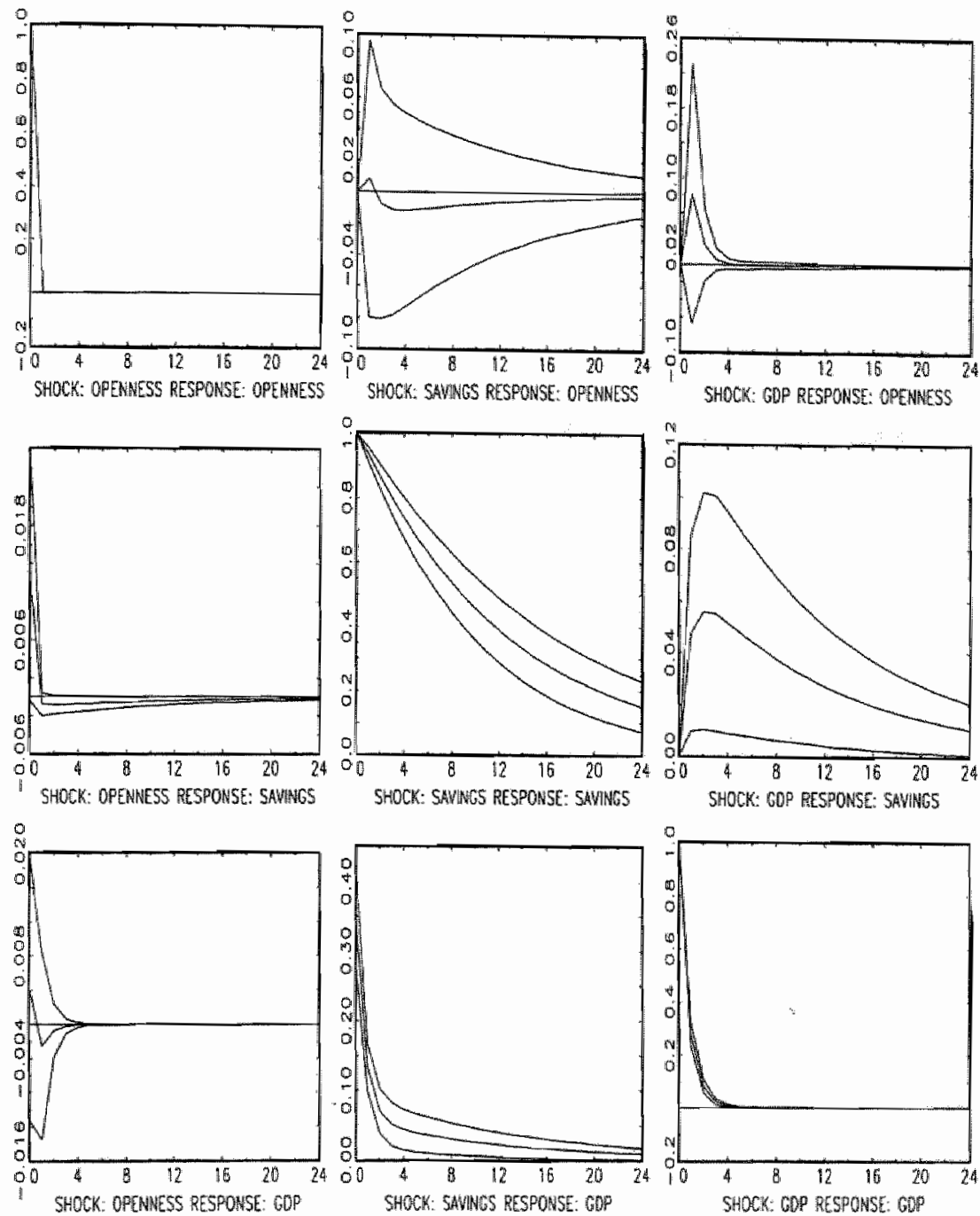

Figure 5.2: Linear impulse response functions for the model with homoscedastic innovations. 

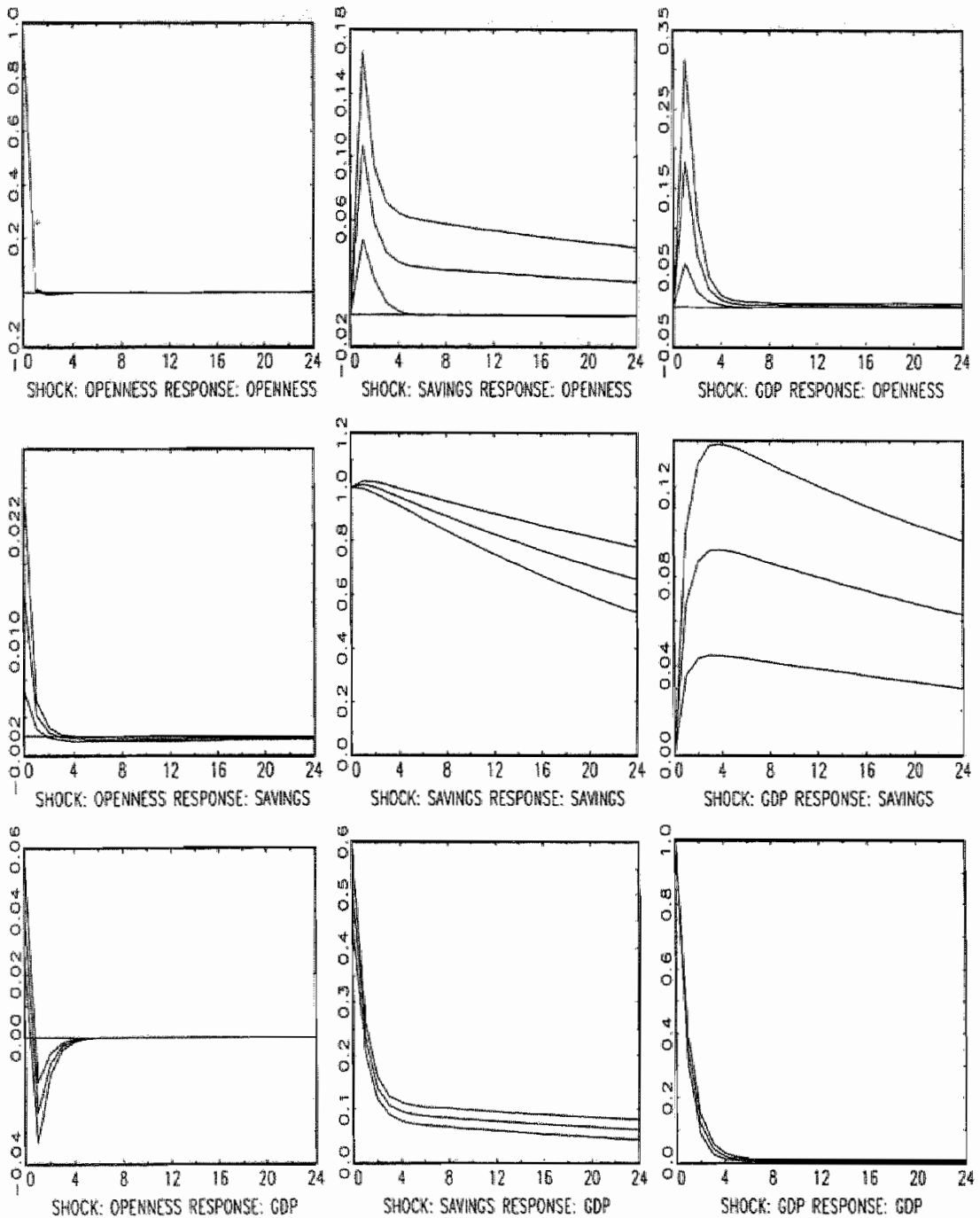

Figure 5.3: Linear impulse response functions for the model with heteroscedastic innovations. 
As expected, in both systems a shock in savings produces a larger reaction of output growth than a shock in openness. This confirms the theoretical notion that the savings level is a more important determinant of output growth than openness. The change in GDP growth in the homoscedastic system to a one time change in openness is insignificant, the response in the heteroscedastic system however is small but significant and negative.

We now analyze the nonlinear impulse responses to a unit shock for both models. We only present the responses to a shock in openness as this is the main interest in this chapter. To get an insight in the behavior of the impulse responses for both systems we simulated 100 sets of parameters from the asymptotic distribution of the estimators. For each one of these sets we generated 50 histories and calculated the impulse responses averaged over 100 futures ( see discussion in section 5.3.3). To keep the analysis more ciear we analyze here the results for the model with the heteroscedastic innovations. The figures for the homoscedastic model are given and analyzed in appendix B.

Figures 5.4 to 5.6 present the average nonlinear impulse responses to a unit shock in openness depending on the starting level of savings. Figure 5.4 gives the results unconditional whether the starting level of savings is above or below the threshold, Figure 5.5 conditional on being above and Figure 5.6 conditional on being below the threshold. The bounds presented in the Figures 5.4, 5.5 and 5.6 represent the area where the $95 \%$ most centered realizations were found. The first row gives the responses to a positive shock, while the second row traces the effects of a negative shock. The average impulse response with 2 standard deviation confidence bounds, based on the same simulation, are given in the Figures 5.7 to 5.9 .

When we compare these impulse response functions with thase given in the linear case in Figure 5.3, we notice that the responses of the savings ratio and openness are of approximately the same size in absolute value. For the nonlinear model we notice, as expected, that the unconditional response is of a magnitude between the two conditional responses. Further we observe clear differences between the responses conditional on a start from a high savings regime and responses originating from the low savings regime. In the high savings regime there is always a positive effect on savings resulting from a positive shock in openness. This in contrast to the response initiated from a low sevings regime where a positive shock initially has a significant negative impact on the saving rate while a negative shock has a positive impact on the saving rate. Note that this strikitig asymmetry of response in the low savings regime cannot be generated by a linear system. Finally notice that the response in the high savings regime is larger than the response resulting from a shock initiated in the low savings regime.

The response of GDP-growth to a positive shock in openness is larger and positive, even after 10 years, in the upper savings regime. In the low savings regime the initially positive response becomes insignificant after 4 years.

Whether we can see the same effects on real countries we calculated the responses for the USA, a notorious low savings country, and Japan with a relatively high savings ratio. We use the parameter estimates and asymptotic distributions from the model under heteroscedasticity as given in Table 5.8. However, for each country we use the most recent years available, 1985-1987, as position at the time of the shock. We simulated 100 sets of parameters and gave both countries a unit shock in openness. The results are shown in the Figures 5.10 to 5.13 . 

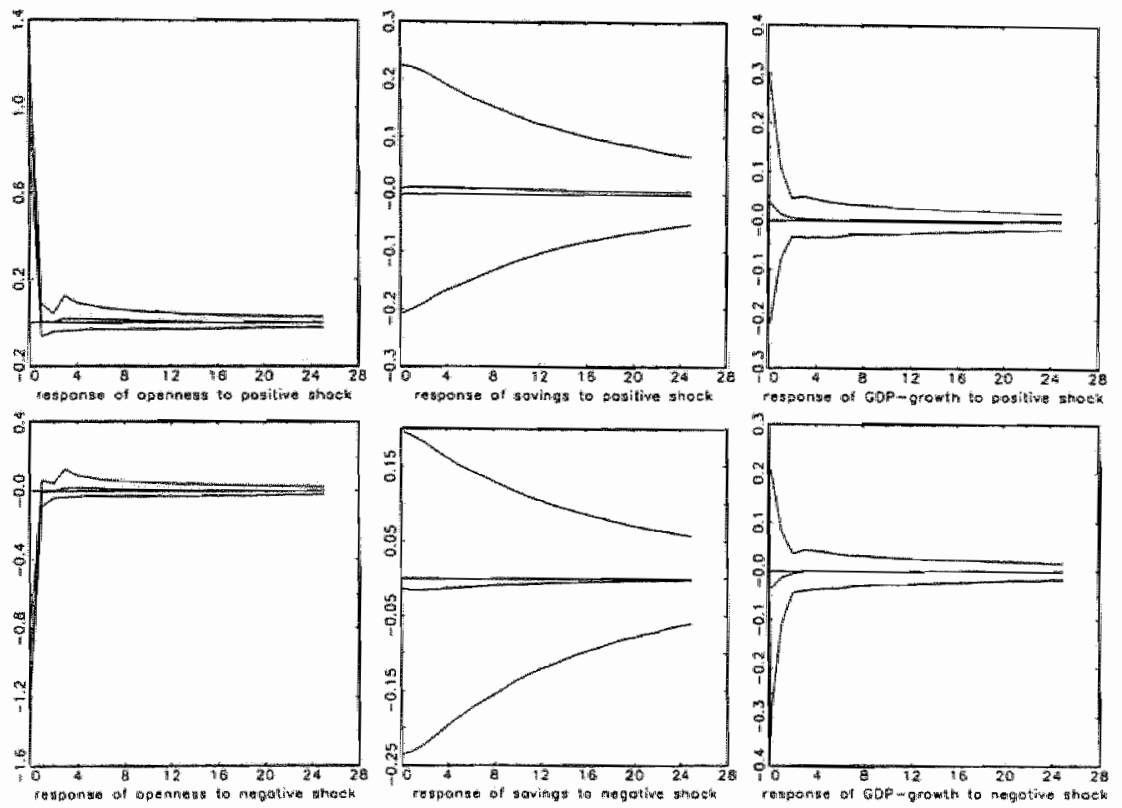

Figure 5.4: Impulse responses to a unit shock in openness, assuming heteroscedastic innovations. Unconditional on the regime. 


\subsection{EMPIRICAL RESULTS}
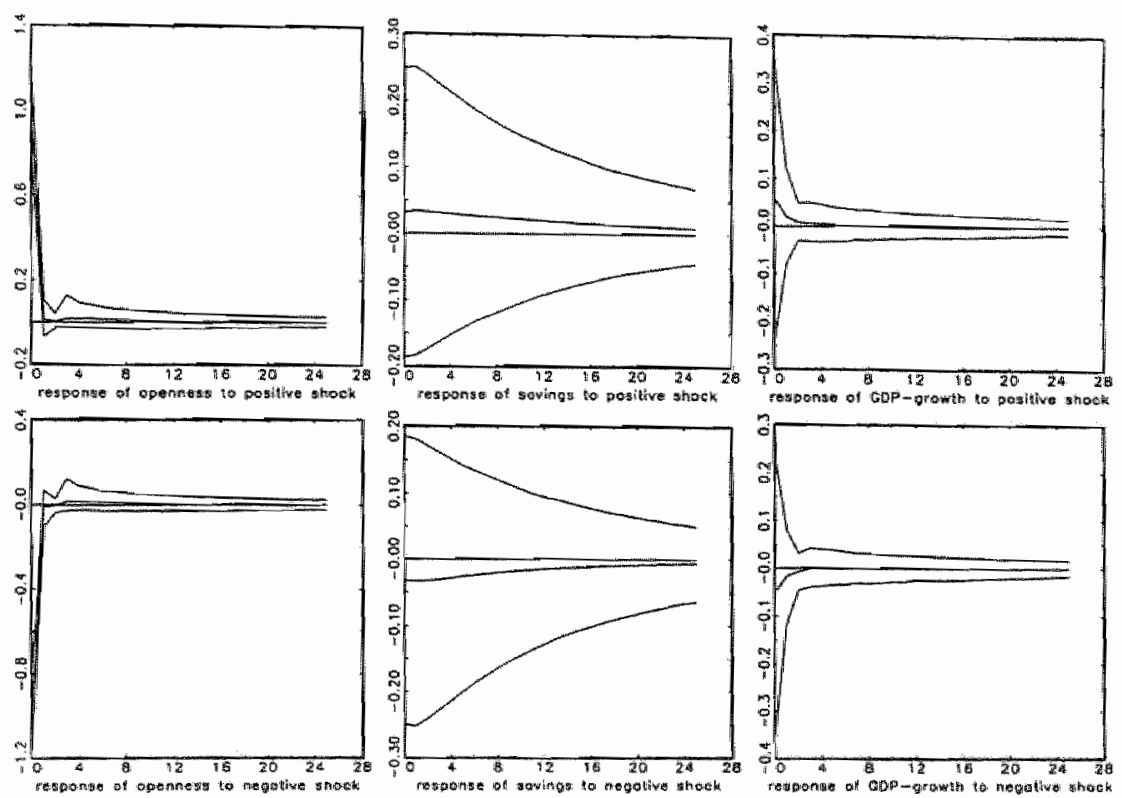

Figure 5.5: Impulse responses ta a unit shock in openness, assuming heteroscedastic innovations. Conditional on being in the upper regime. 

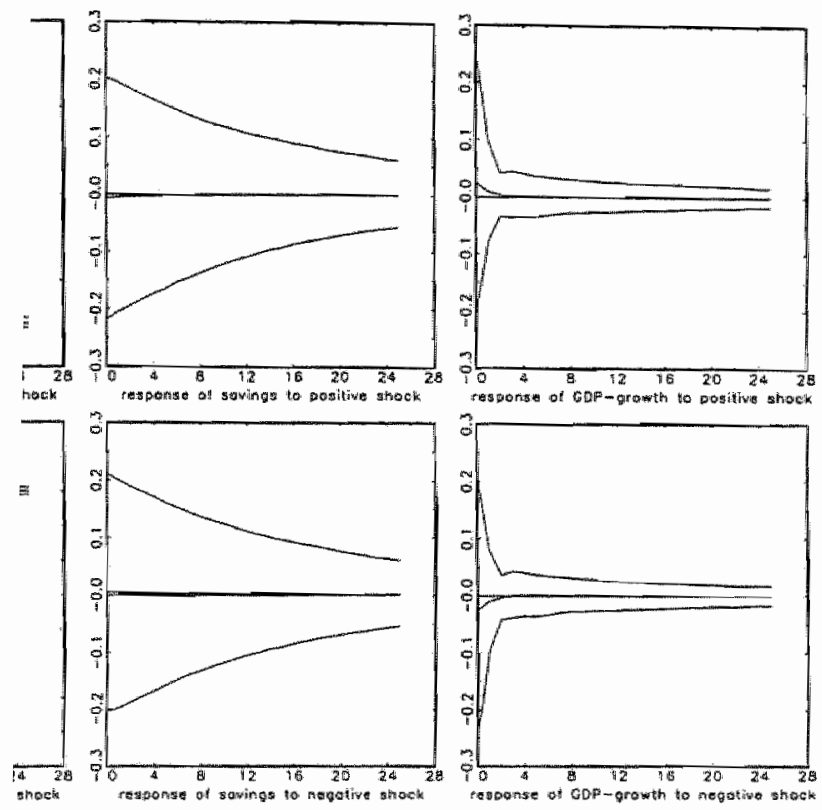

mses to a unit shock in openmess, assuming heteroscedastic in being in the lower regime. 


\subsection{EMPIRICAL RESULTS}
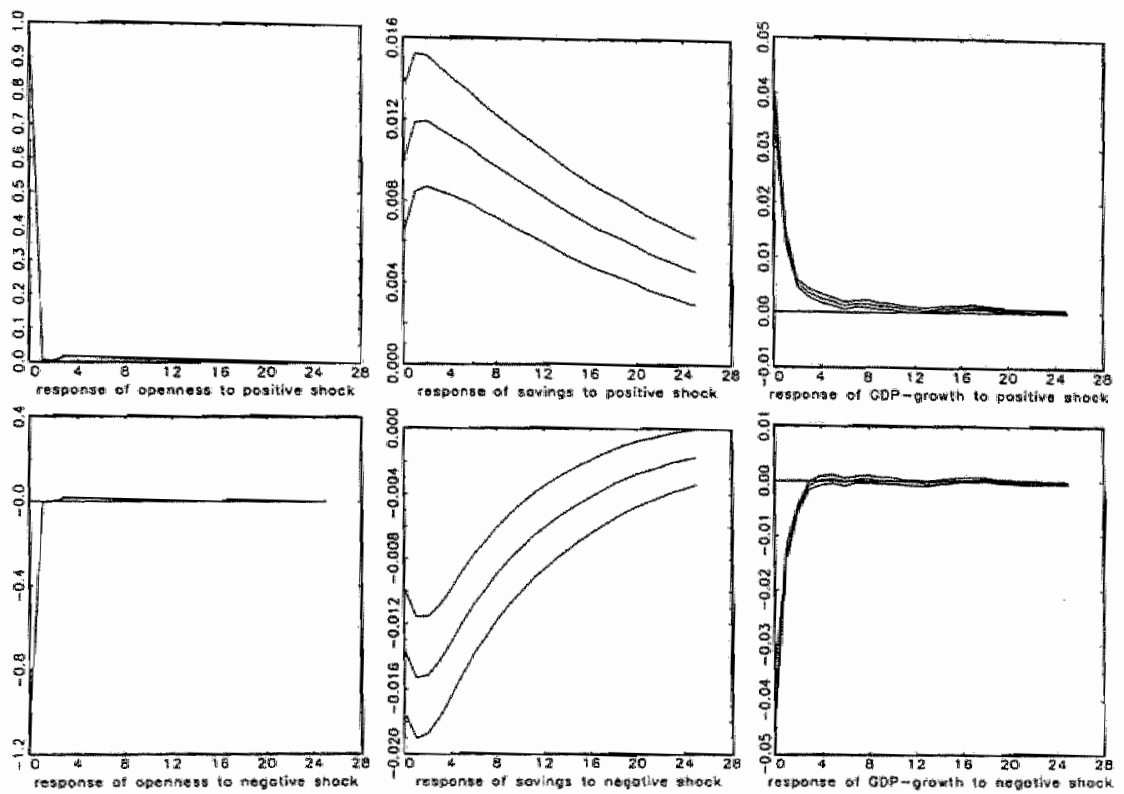

Figure 5.7: Average impulse responses to a unit shock in openness with 2 standarddeviation confidence bounds. Model with heteroscedastic immovations. Unconditional on regime. 

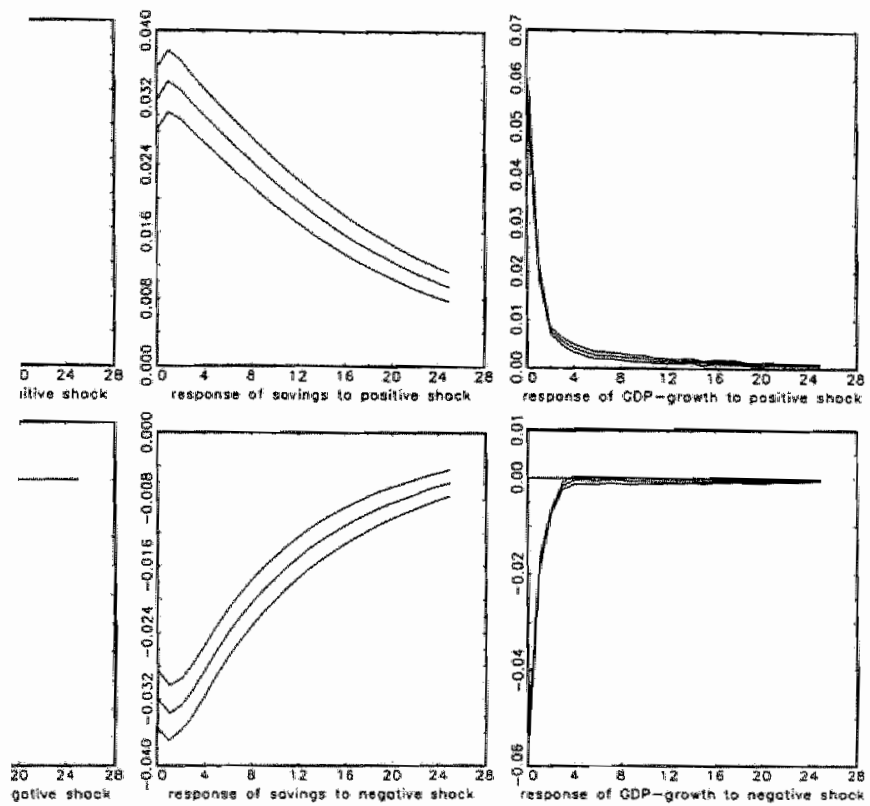

mpulse responses to a unit shock in openness with 2 standardsounds. Model with heteroscedastic innovations. Conditional on 


\subsection{EMPIRICAL RESULTS}
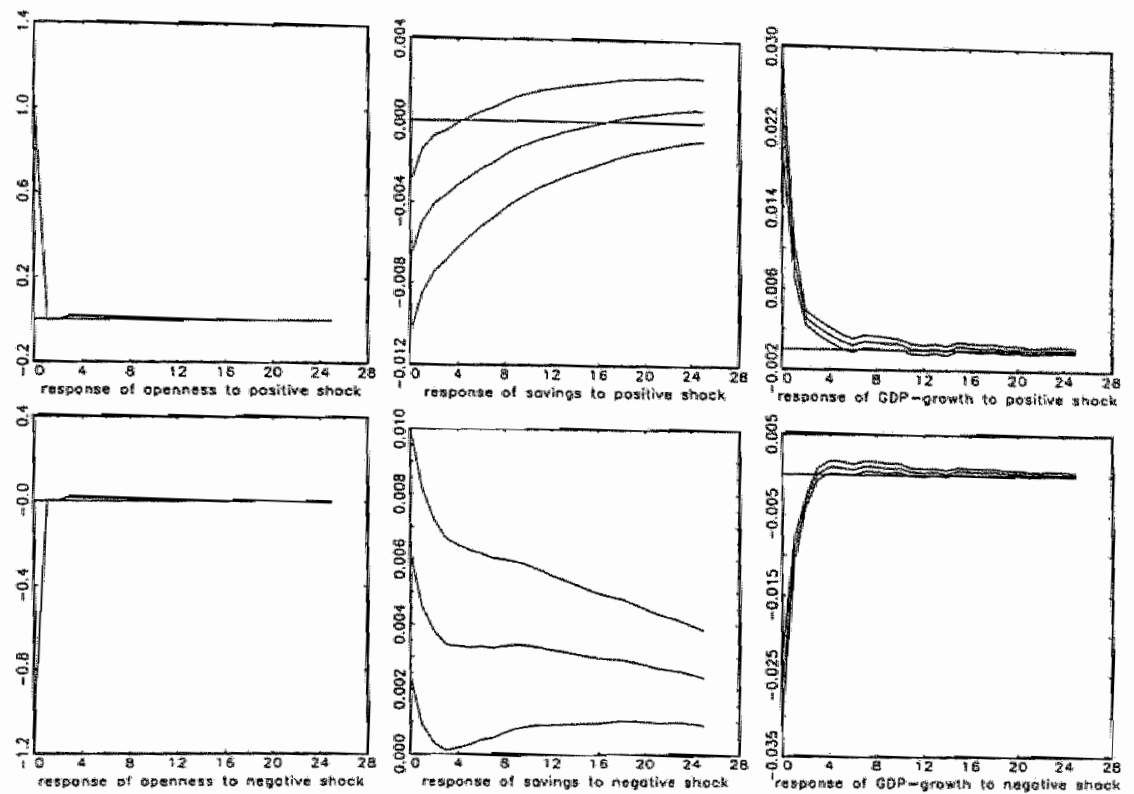

Figure 5.9: Average impulse responses to a unit shock in openness with 2 standarddeviations confidence bounds. Model with heteroscedastic innovations. Conditional on being in lower regime. 

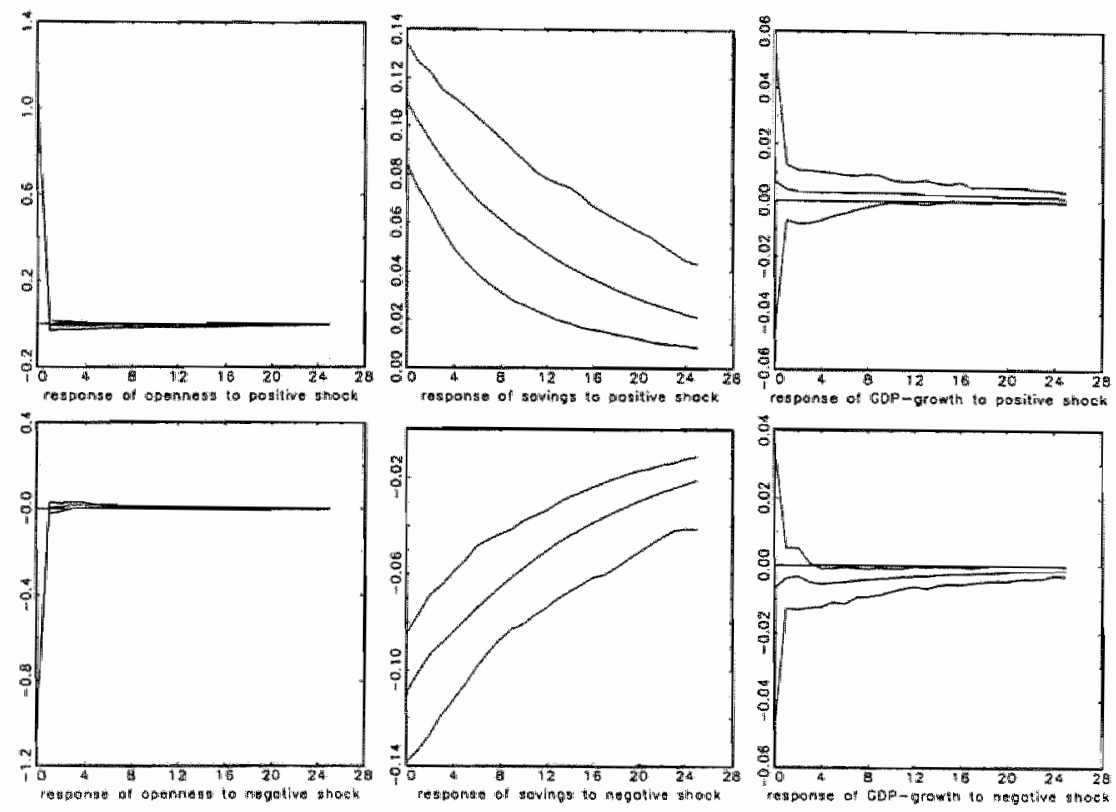

Figure 5.10: Impulse responses to a unit shock in openness for Japan based on the model with heteroscedastic innovations. 

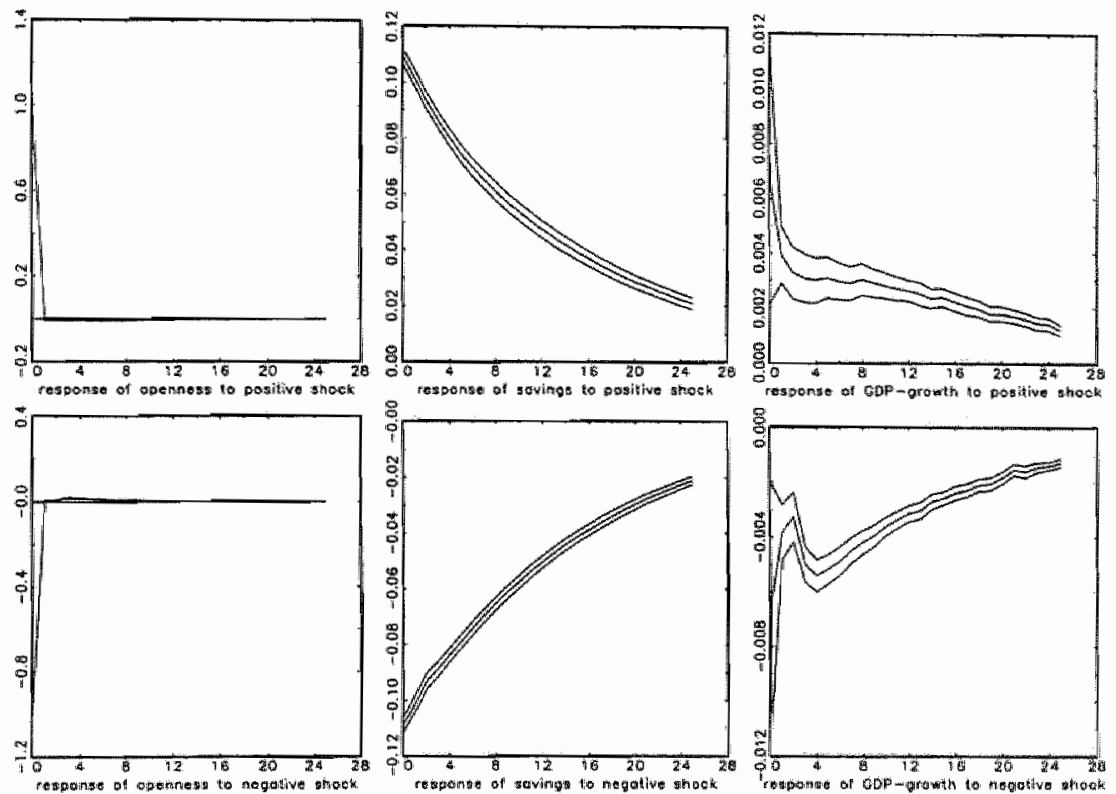

Figure 5.11: Average impulse response to a unit shock in openness for Japan. Model with heteroscedastic innowations. 

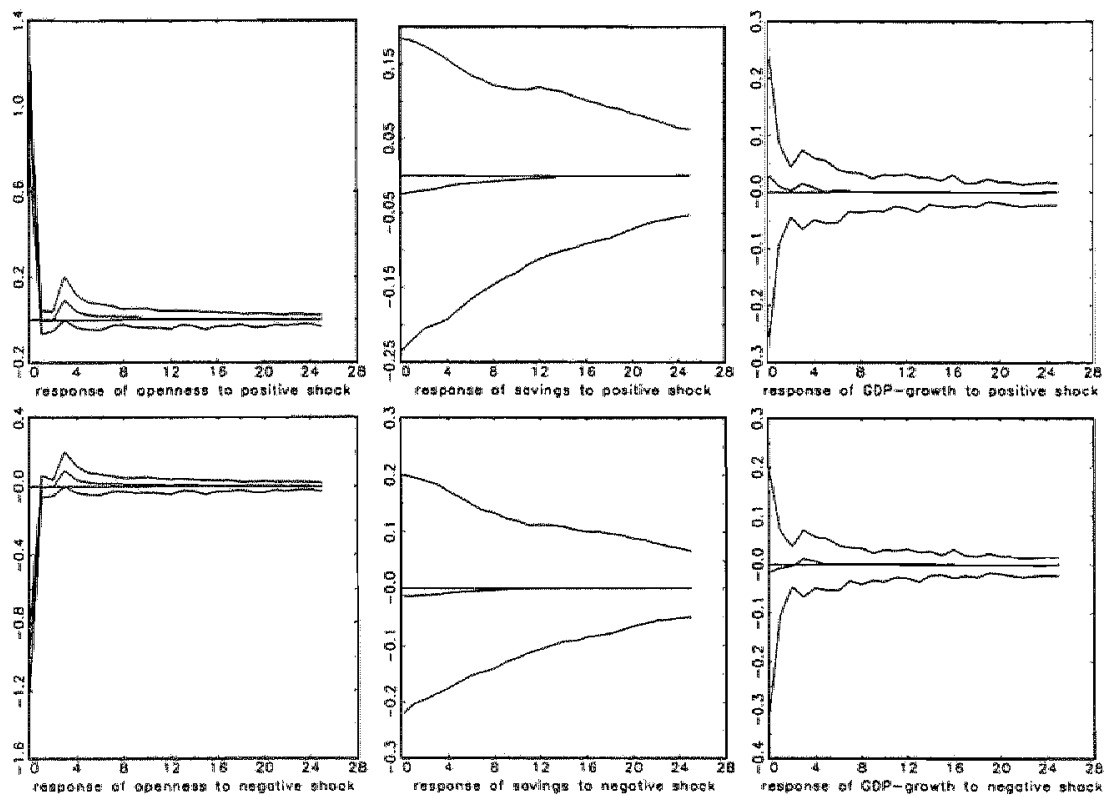

Figure 5.12: Impulse responses to a unit shock in openness for the USA based on the model with heteroscedastic innovations. 

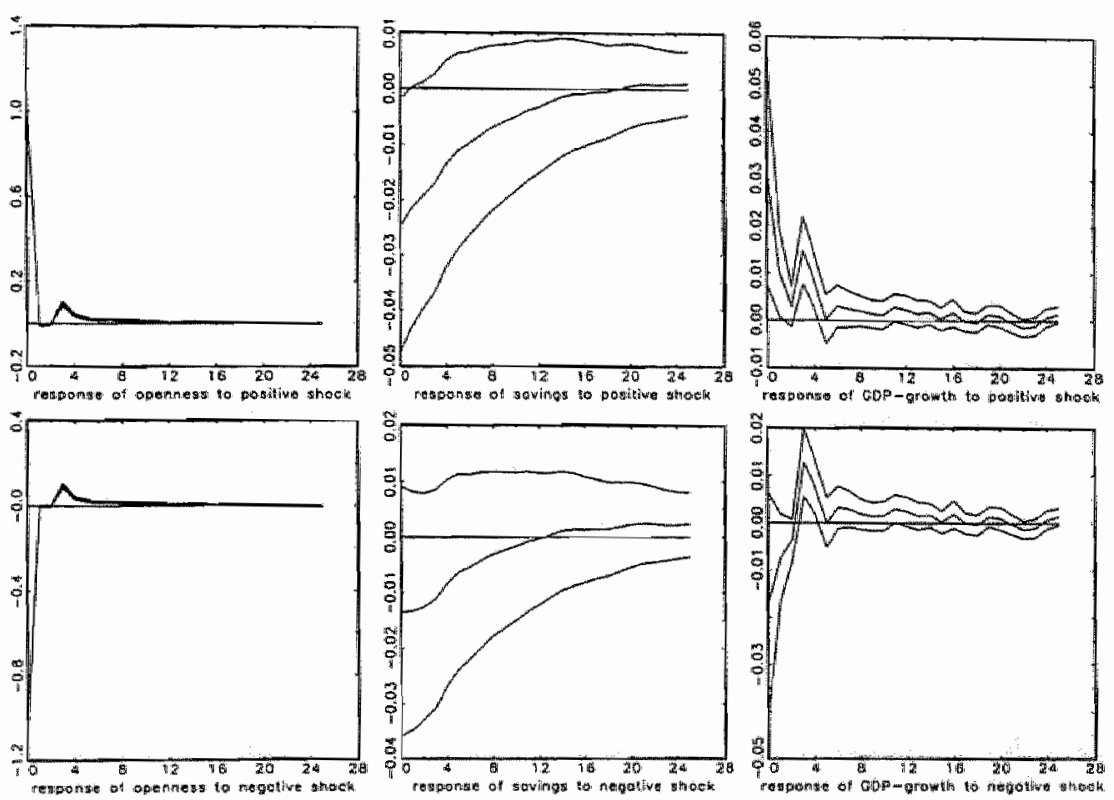

Figure 5.13: Average impulse response to a unit shock in openness for the USA. Model with heteroscedastic innovations. 
First we see that Japan's savings ratio reacts with a significant positive response while the savings ratio of the USA does give a significant response. Further we see that the GDP-growth of Japan is positively influenced by a positive shock in openness. This in contrast to the USA where only the third year a positive effect is found.

\subsection{Conclusions}

In this chapter we test a simple hypothesis: does a country's saving rate matter both over time and across countries for the interaction between growth and openness? Using a dynamic model for GDP growth, the saving rate and openness to trade we find that it indeed matters. Countries with high saving rates experience a growth-enhancing effect from an increase in openness while countries with low saving rates experience a decrease in sawings and hardly any change GDP growth. First, the differences between low and high saving rate countries are evident from the estimation of the threshold VAR models. In the high savings regime, change in openness has a positive effect on growth, while the same effect in the low savings regime is insignificant. Second, there are striking differences in the impulse response functions between the two regimes. In the high savings regime, a positive shock in openness leads to higher GDP growth in subsequent years, while in the low savings regime, the initially positive GDP growth rate becomes insignificant after a few years.

Our empirical findings have nontrivial policy implications. Clearly, low saving rates are a double curse for a country. On the one hand, a low national saving rate directly diminishes the domestic growth fundamentals of the economy. Furthermore, as our analysis indicates, it also undermines the potential growth effects of increased openness to world trade experienced by high savings countries. Even worse, for some countries a higher volume of trade may even reduce the GDP growth rate which is already relatively due to the low national saving rate.

In addition to the empirical results, the paper also adds to the theoretical econometric literature by introducing a new simple test for endogenous threshold models. The test has the advantage that it can be calculated efficiently, especially in larger data sets. Another advantage is that the test can be used without a large number of tables with critical values at hand. A less desirable property of the test is that it can be more conservative than other known tests such as the exponential-, average-, and supremum-LM test. 


\subsection{Appendix A}

In the tables below we present the results for the equations of the VAR with homogeneous innovations when the threshold is not restricted to be the same for each equation.

\begin{tabular}{|c|c|c|c|c|c|c|}
\hline & \multicolumn{6}{|c|}{ dependent variables } \\
\hline & \multicolumn{2}{|c|}{$\Delta y_{i, t}$} & \multirow{2}{*}{\multicolumn{2}{|c|}{$\frac{S_{i, t}}{0.196}$}} & \multirow{2}{*}{\multicolumn{2}{|c|}{$\frac{\Delta O_{i, t}}{0.162}$}} \\
\hline$\gamma$ & & & & & & \\
\hline regressors & $S_{i, t-2} \leq \gamma$ & $S_{i, t-2}>\gamma$ & $S_{i, t-2} \leq \gamma$ & $\overline{S_{i, t-2}>\gamma}$ & $S_{i, t-2} \leq \gamma$ & $\overline{S_{i, t-2}>\gamma}$ \\
\hline$c$ & $\begin{array}{l}0.004 \\
(0.003)\end{array}$ & $\begin{array}{l}0.010 \\
(0.011)\end{array}$ & $\begin{array}{l}0.022 \\
(0.003)\end{array}$ & $\begin{array}{l}0.017 \\
(0.004)\end{array}$ & $\begin{array}{l}0.012 \\
(0.017)\end{array}$ & $\begin{array}{c}-0.002 \\
(0.013)\end{array}$ \\
\hline$S_{i, t}$ & $\begin{array}{l}0.307 \\
(0.036)\end{array}$ & $\begin{array}{l}0.535 \\
(0.072)\end{array}$ & - - & - & - & \\
\hline$\Delta O_{i, t}$ & $\begin{array}{l}0.007 \\
(0.009)\end{array}$ & $\begin{array}{l}-0.049 \\
(0.023)\end{array}$ & $\begin{array}{l}-0.010 \\
(0.009)\end{array}$ & $\begin{array}{l}0.068 \\
(0.010)\end{array}$ & - & - \\
\hline$\Delta y_{i, t-1}$ & $\begin{array}{l}0.345 \\
(0.028)\end{array}$ & $\begin{array}{l}0.079 \\
(0.045)\end{array}$ & $\begin{array}{l}0.047 \\
(0.031)\end{array}$ & $\begin{array}{l}0.044 \\
(0.023)\end{array}$ & $\begin{array}{c}-0.317 \\
(0.169)\end{array}$ & $\begin{array}{l}0.255 \\
(0.077)\end{array}$ \\
\hline$S_{i, t-1}$ & $\begin{array}{c}-0.252 \\
(0.037)\end{array}$ & $\begin{array}{c}-0.468 \\
(0.077)\end{array}$ & $\begin{array}{l}0.864 \\
(0.024)\end{array}$ & $\begin{array}{l}0.918 \\
(0.017)\end{array}$ & $\begin{array}{l}0.320 \\
(0.147)\end{array}$ & $\begin{array}{l}0.007 \\
(0.054)\end{array}$ \\
\hline $\bar{N}$ & 1108 & 400 & 711 & 797 & 477 & 1031 \\
\hline$\overline{R^{2}}$ & 0.19 & 0.13 & 0.68 & 0.79 & 0.01 & 0.01 \\
\hline
\end{tabular}

Table 5.9: Estimation results for VAR with homoscedastic innovations, with no crossequation restrictions on the threshold.

\subsection{Appendix B}

In this appendix we give the nonlinear impulse responses for the model with homoscedastic disturbances. We do not give an explicit analysis but the reader is invited to draw his own conclusions. In our opinion the model with homoscedastic disturbances is less robust and has some features of non-stationarity. We present the results here to show that nonlinear threshold models can exhibit asymmetries are not found in linear VAR's.

In the main text we presented the impulse responses resulting from a unit shock in openness. Here we present impulse responses resulting from a one standard-deviation shock in openness. 

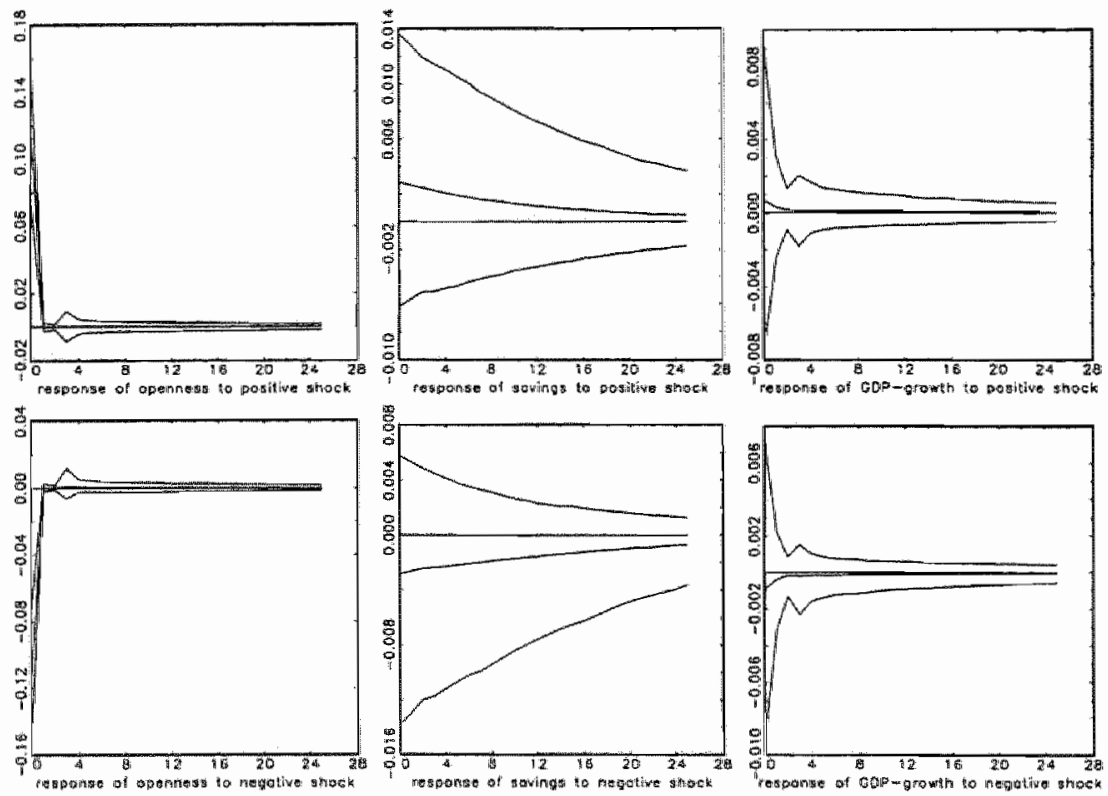

Figure 5.14: Impulse responses to ane standard-deviation shock in openness assuming homoscedastic innovations. Unconditional on the regime. 

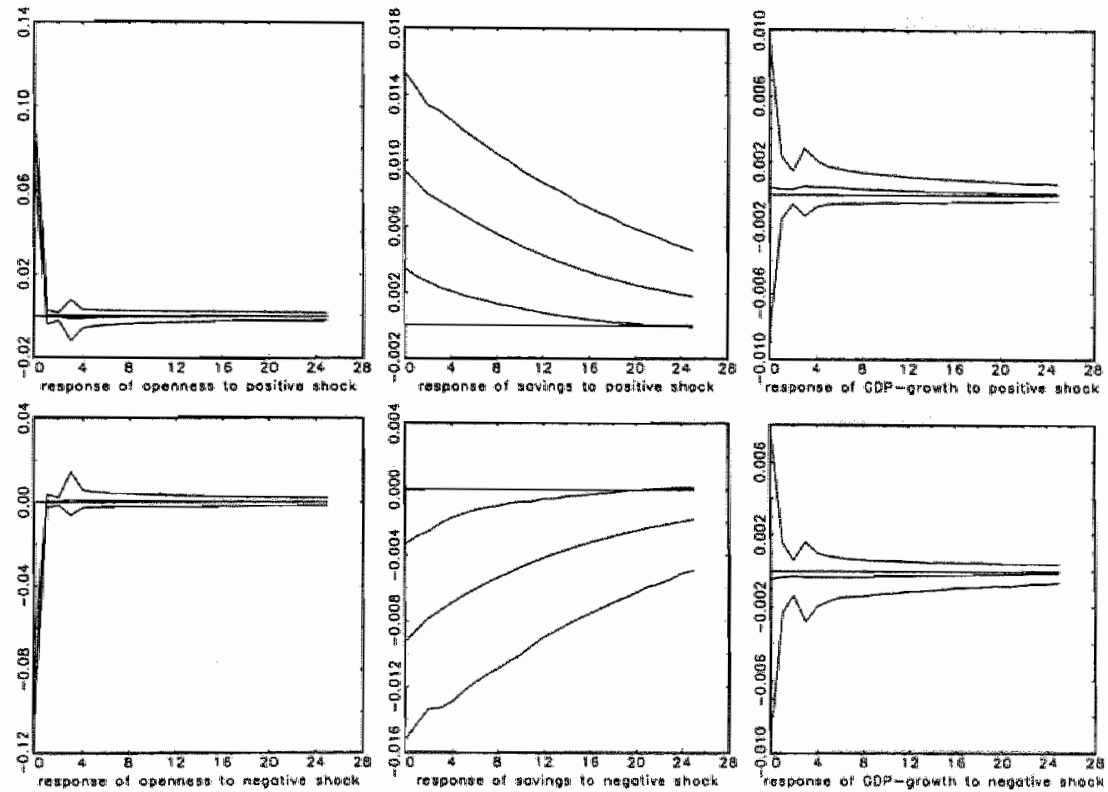

Figure 5.15: Impulse responses to a one standard-deviation shock in openness assuming homoscedastic innovations. Conditional on being in the upper regime. 

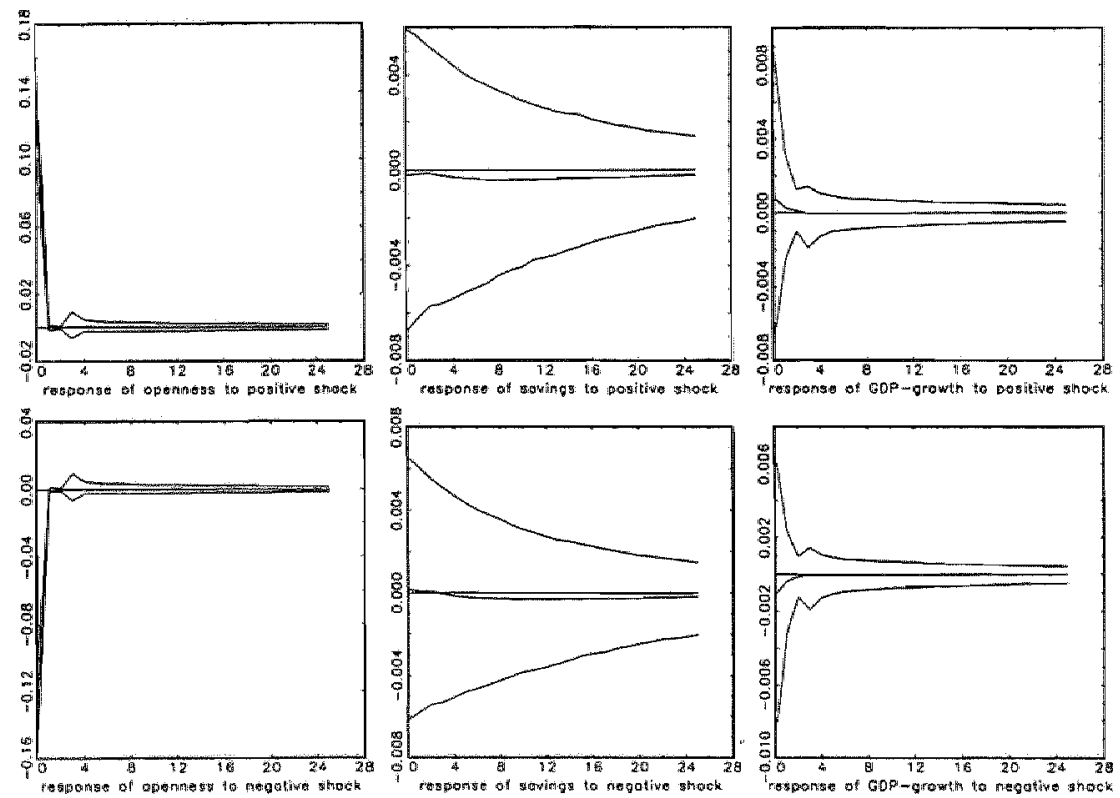

Figure 5.16: Impulse responses to a one standard-deviation shock in openness assuming homoscedastic innovations. Conditional on being in the lower regime. 

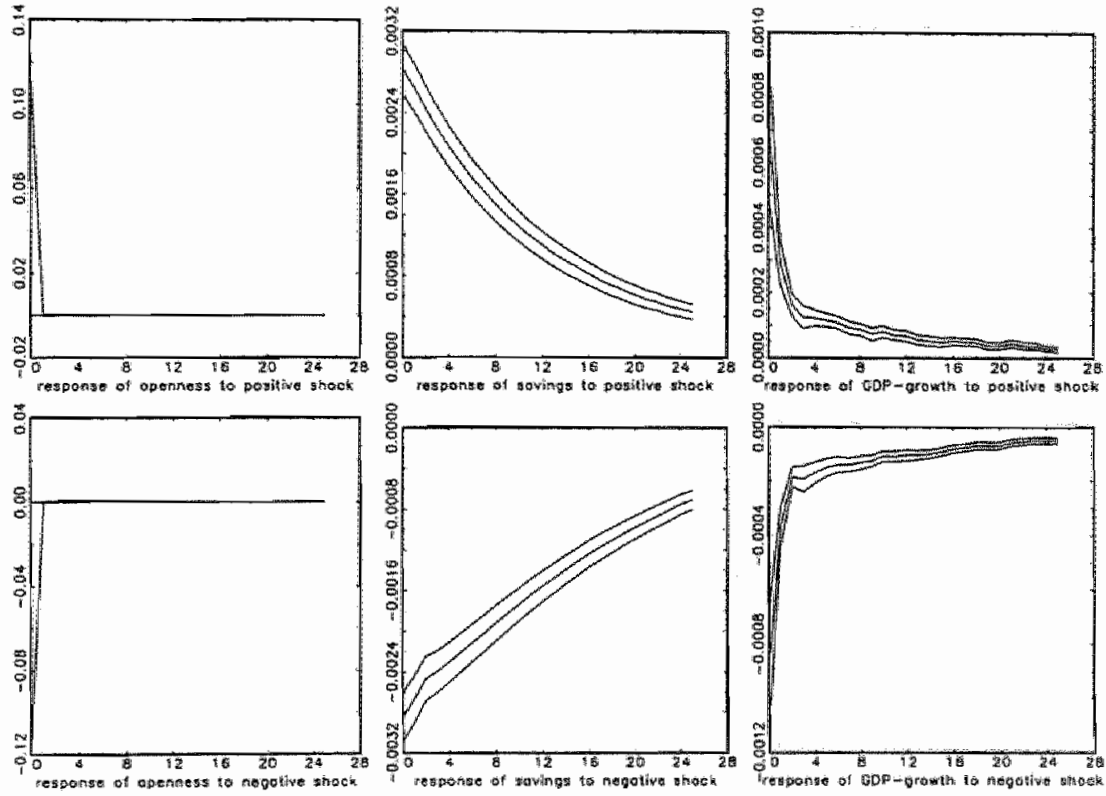

Figure 5.17: Average impulse response to a one standard-deviation shock in openness with 2 standard deviation confidence bounds. Model with homoscedastic innovations. Unconditional on regime. 

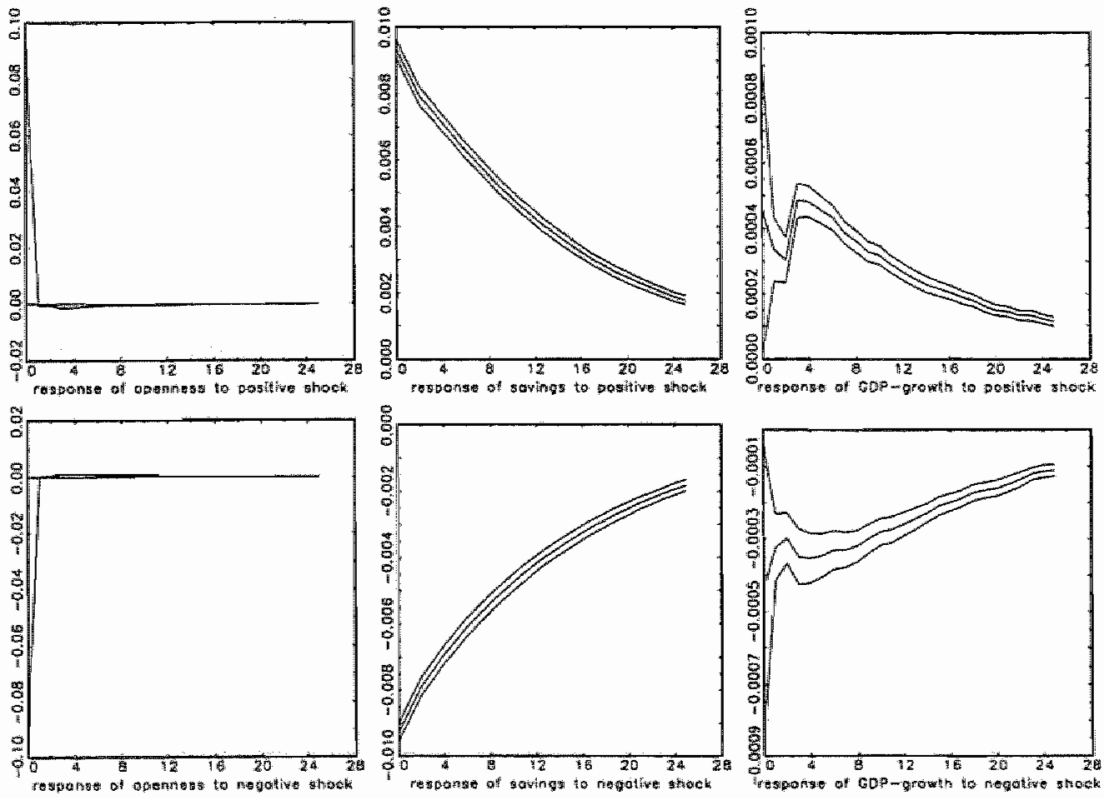

Figure 5.18: Average impulse response to a one standard-deviation shock in openness with 2 standard deviation confidence bounds. Model with homoscedastic innovations. Conditional on being in upper regime. 

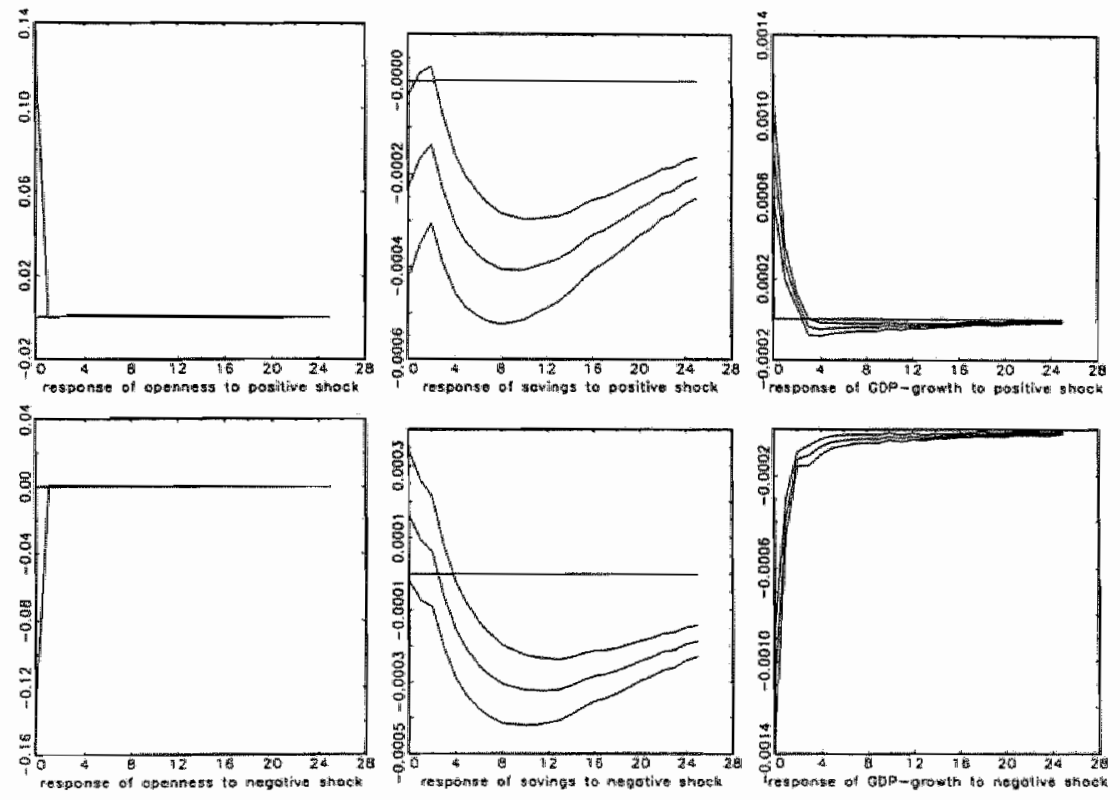

Figure 5.19: Average impulse response to a one standard-deviation shock in openness with 2 standard deviation confidence bounds. Model with homoscedastic innovations. Conditional on being in lower regime. 

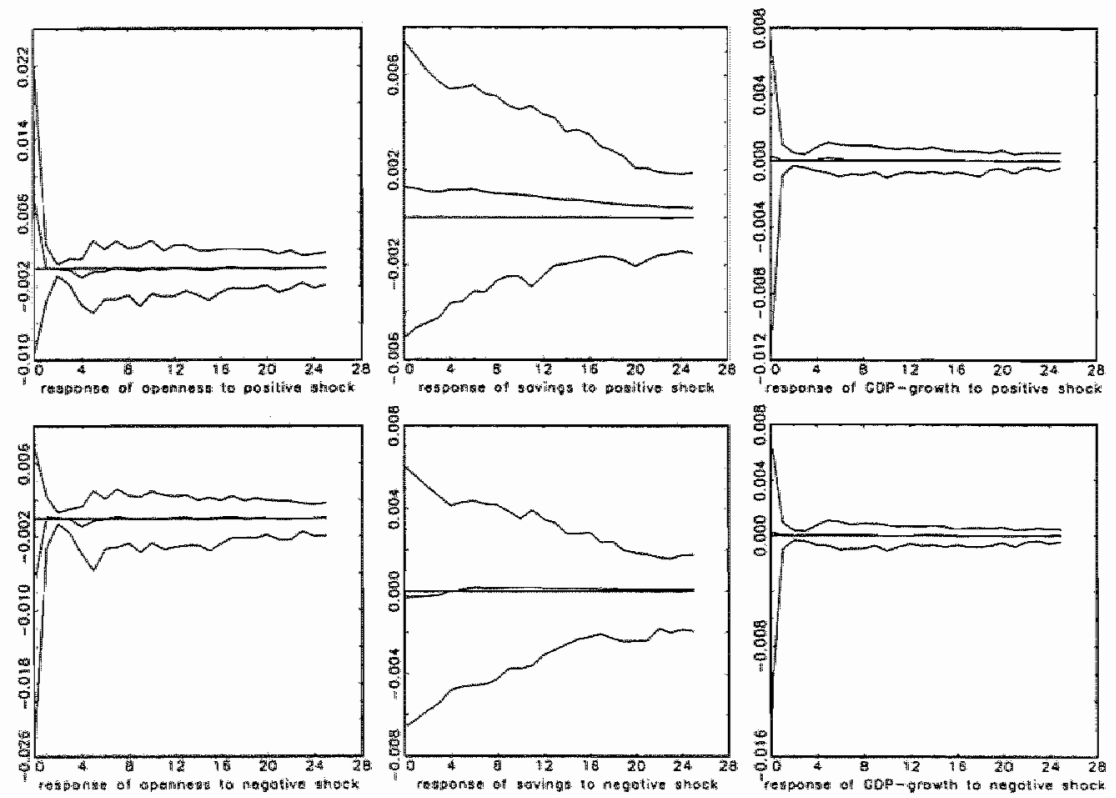

Figure 5.20: Impulse responses to a one standard-deviation shock in openness for Japan based on the model with homoscedastic innovations. 

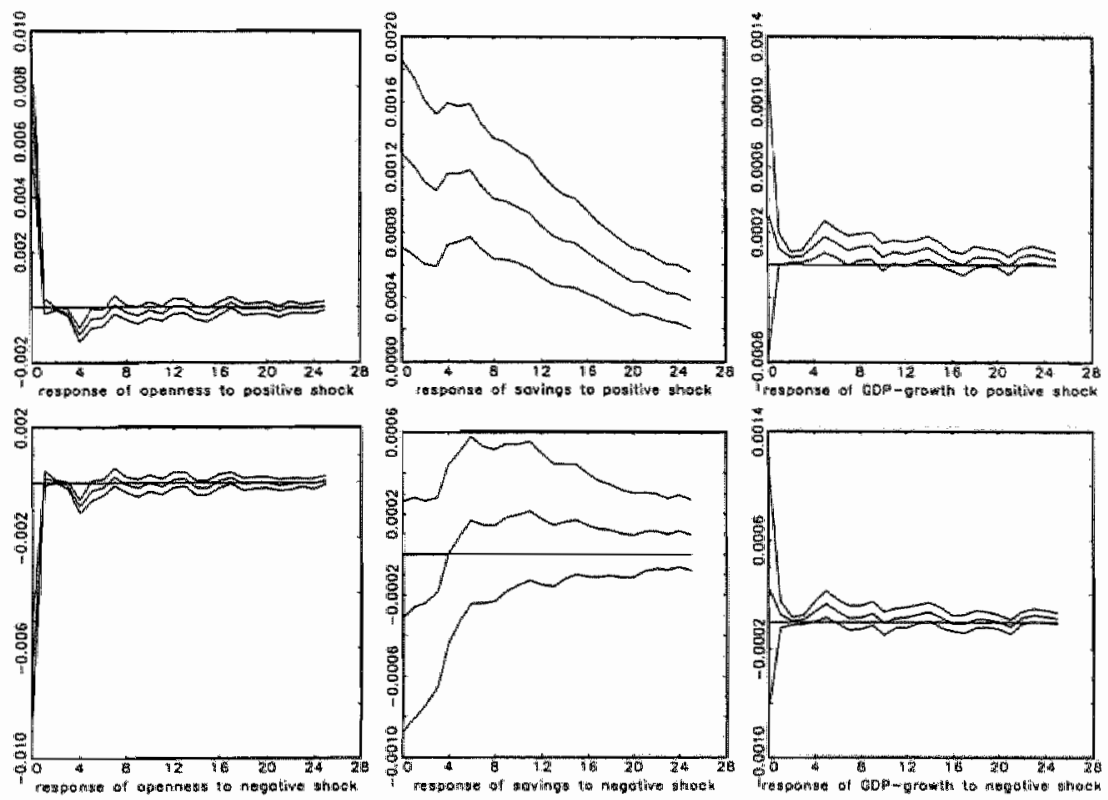

Figure 5.21: Average impulse response to a one standard-deviation shock in openness for Japan. Model with homoscedastic innovations. 

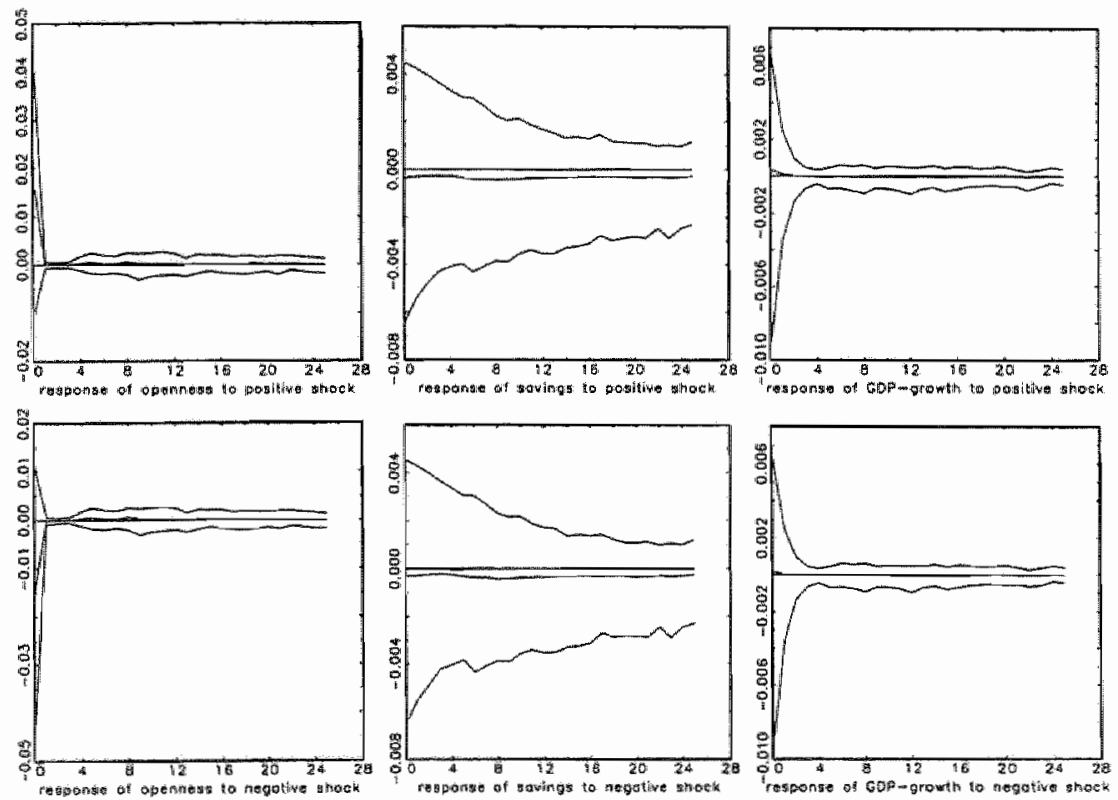

Figure 5.22: Impulse responses to a one standard-deviation shock in openness for the USA based on the model with homoscedastic innovations. 

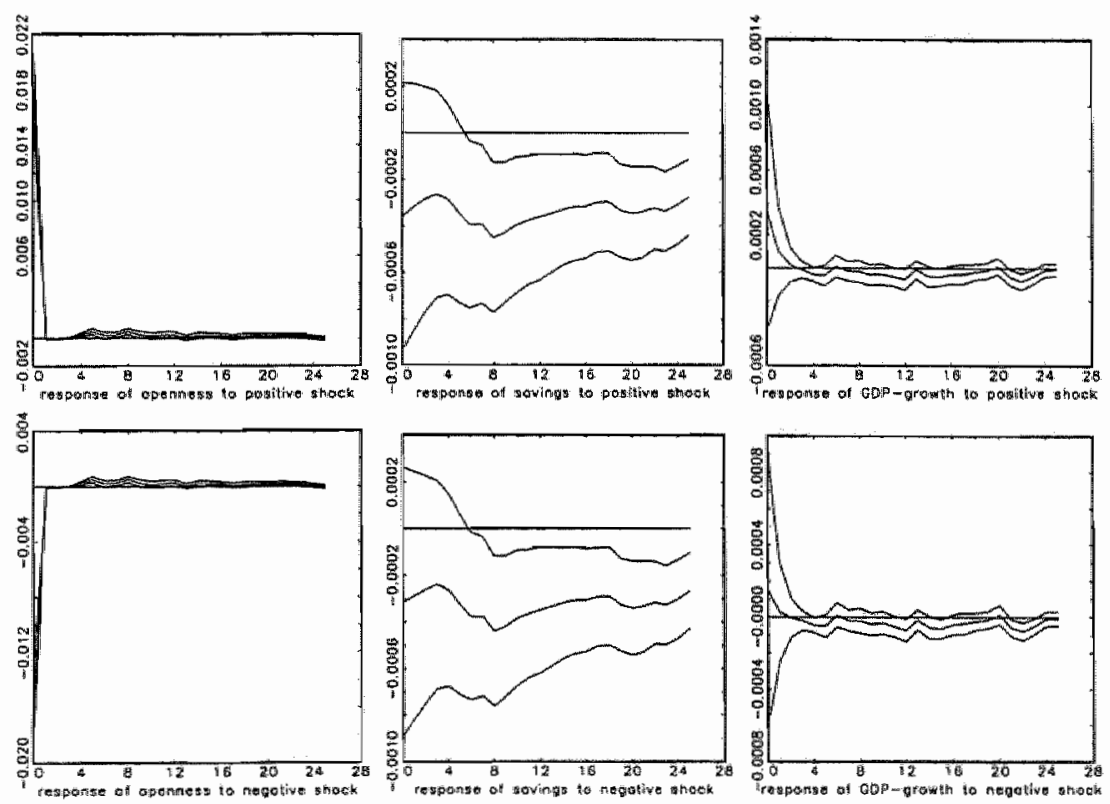

Figure 5.23: Average Impulse response to a one standard-deviation shock in openness for the USA. Model with homoscedastic innovations. 


\subsection{Appendix C}

Using the notation as in section 3.1 we observe that we have introduced the test for the existence of a threshold making use of the restricted estimator. We will now first show that we can also use the unrestricted estimator to obtain a test for $\beta_{2}=0$.

Introducing the projection matrix $M=\left(I-X\left(X^{\prime} X\right)^{-1} X^{\prime}\right)$, we have by the FrischWaugh-Lovell theorem, see for instance Davidson and MacKinnon (1993) as consistent estimator of $\beta_{2}$ for fixed scalar $\gamma$

$$
\hat{\beta}_{2}(\gamma)=\left(Z_{(\gamma)}^{\prime} M Z_{(\gamma)}\right)^{-1} Z_{(\gamma)}^{\prime} M Y
$$

Then we observe that

$$
z_{N}(\gamma)=\frac{1}{\sqrt{N}}\left(Z_{(\gamma)}^{\prime} M Z_{(\gamma)}\right) \hat{\beta}_{2}(\gamma)=\frac{1}{\sqrt{N}} Z_{(\gamma)}^{\prime} M Y
$$

which implies

$$
\begin{array}{ll}
Z_{(\gamma)}^{\prime} M Y=Z_{(\gamma)}^{\prime} M \varepsilon & \text { under } H_{0} \\
Z_{(\gamma)}^{\prime} M Y=Z_{(\gamma)}^{\prime} M Z_{(\gamma)} \beta_{2}+Z_{(\gamma)}^{\prime} M \varepsilon & \text { under } H_{1} .
\end{array}
$$

So we can again define the integrals as given in the main text. Observe that in this case we use the unrestricted estimator of the parameters, while in the main text we use the test based on the restricted estimator. It follows directly from (5.25) and (5.26) that their asymptotic behavior will be the same.

Before we derive the asymptotic distribution of the test statistic first some remarks concerning the power of the test. In the empirical work in section 5 we observed that our test, the LM version of the test, has less power than the other LM tests. To investigate in more detail this phenomenon we consider the model

$$
Y=X \beta_{1}+Z_{(r)} \beta_{2}+u,
$$

with $u \sim N\left(0, \sigma^{2} I\right)$. For fixed $\gamma$ the LM statistic for the hypothesis $\beta_{2}=0$ is given by

$$
\begin{aligned}
L M(\gamma) & =\frac{\left(Y-X \hat{\beta}_{1}\right)^{\prime} Z_{(\gamma)}\left(Z_{(\gamma)}^{t} M Z_{(\gamma)}\right)^{-1} Z_{(\gamma)}^{\prime}\left(Y-X \tilde{\beta}_{1}\right)}{Y^{\prime} M Y} \\
& =\frac{u^{r^{\prime}} Z_{(\gamma)}\left(Z_{(\gamma)}^{\prime} M Z_{(\gamma)}\right)^{-1} Z_{(\gamma)}^{\prime} u^{r}}{\hat{\sigma^{2}}},
\end{aligned}
$$

where $\widetilde{\beta}_{1}$ is the estimator based on the restricted estimate, $M=\left(I-X\left(X^{\prime} X\right)^{-1} X^{\prime}\right)$ and $u^{r}$ the residual based on $\tilde{\beta}_{1}$. Observe that the following relations hold, $u^{r}=M Y=M u$ which gives under $H_{0}$

$$
\begin{aligned}
L M(\gamma) & =\frac{u^{r^{\prime}} M Z_{(\gamma)}\left(Z_{(\gamma)}^{\prime} M Z_{(\gamma)}\right)^{-1} Z_{(\gamma)}^{\prime} M u^{r}}{\hat{\sigma^{2}}} \\
& =\frac{1}{\hat{\sigma^{2}}} \operatorname{trace}\left(\left(Z_{(\gamma)}^{\prime} M Z_{(\gamma)}\right)^{-1} Z_{(\gamma)}^{\prime} M u u^{\prime} M Z_{(\gamma)}\right) .
\end{aligned}
$$


The relation between our test statistic and the exponential-, average- and supremum LM statistics can now be explained easily. All test statistics are based on functionals of $L M(\gamma)$. Our test makes use of the following functional

$$
\frac{1}{\sigma^{2}} \mathcal{S}\left(z_{N}(\gamma) z_{N}^{\prime}(\gamma)\right)=\frac{1}{\sigma^{2}} \mathcal{S}\left(Z_{(\gamma)}^{r} M u u^{\prime} M Z_{(\gamma)}\right)
$$

where we have used $\hat{\sigma}^{2}$ from the denominator in (5.14). Now we can easily explain the relation between (5.28) and the LM statistic. The normalization in (5.27) by $\left(Z_{(\gamma)}^{\prime} M Z_{(\gamma)}\right)^{-1}$ transforms the LM statistic to a standard normalized chi-squared statistic, which is independent of the data. Under the null hypothesis we have a sum of independent squared normal random variables. We therefore only have to consider the trace of the matrix. As this transformation makes the LM statistic independent of the data asymptotic distributions from functionals of the LM are also independent of the data. This means that critical values can be tabulated. Observe however that usually the asymptotic distribution depends on the number of regressors.

In (5.28) we see that we do not use this normalization. This means $\frac{1}{\sigma^{2}} \mathcal{S}\left(z_{N}(\gamma) z_{N}^{t}(\gamma)\right)$ still depends on the data by $\left(Z_{(\gamma)}^{\prime} M Z_{(\gamma)}\right)^{-1}$. Further as the matrix does not become diagonal under the null hypothesis we also have to take into account the off diagonal elements. We therefore introduced the $\mathcal{S}(\bullet)$ operator.

As $\frac{1}{\hat{\alpha}} \mathcal{S}\left(z_{N}(\gamma) z_{N}^{\prime}(\gamma)\right)$ depends on the data so does $\int \frac{1}{\hat{\alpha}} \mathcal{S}\left(z_{N}(\gamma) z_{N}^{\prime}(\gamma)\right) d \gamma$,

However below it is shown that a data independent upperbound for the asymptotic distribution of $\int \frac{1}{\hat{\sigma^{2}}} \mathcal{S}\left(z_{N}(\gamma) z_{N}^{\prime}(\gamma)\right) d \gamma$ can be given. An additional advantage is that the number of regressors does not affect the asymptotic distribution. Using an upperbound automatically implies that the test statistic will exhibit some conservatism. The degree of conservatism depends on the data. Finally observe that both test statistics will asymptotically be the same if $\left(Z_{(\gamma)}^{\prime} M Z_{(\gamma)}\right)=I_{k_{z}}$.

We now concentrate on the validity of our claims in Section 3.1. Using the notation from Section 3.1 again we observe

$$
\begin{array}{rlrl}
z_{N}(\gamma) & =\frac{1}{\sqrt{N}} Z_{(\gamma)}^{\prime} u^{r}=\frac{1}{\sqrt{N}} Z_{(\gamma)}^{\prime} M \varepsilon & \text { under } & H_{0} \\
z_{N}(\gamma) & =\frac{1}{\sqrt{N}} Z_{(\gamma)}^{\prime} u^{r}=\frac{1}{\sqrt{N}} Z_{(\gamma)}^{\prime} M Z_{(\gamma)} \beta_{2}+\frac{1}{\sqrt{N}} Z_{(\gamma)}^{\prime} M \varepsilon & \text { under } \quad H_{1}
\end{array}
$$

Under the assumptions we set out below we have that $\frac{1}{\sqrt{N}} Z_{(\gamma)}^{\prime} M \varepsilon$ converges weakly to a. zero mean $k_{z^{-}}$dimensional Gaussian process. This means that under $H_{0}$ the process $z_{N}(\gamma)$ is also a Gaussian process with zero mean. Under $H_{1}$ however there is still the term $\frac{1}{\sqrt{N}} Z_{(\gamma)}^{\prime} M Z_{(\gamma)} \beta_{2}$. Under the assumptions we set we have for fixed $\gamma$,

$$
\lim _{N \rightarrow \infty} \frac{1}{N} Z_{(\gamma)}^{s} M Z_{(\gamma)}=\sigma^{2} \Omega_{(\gamma)}
$$

and this means that when $\beta_{2} \neq 0$, this term diverges to plus or minus infinity. This fact will make our test consistent. However we can use this also to say a little bit more. 
We can also consider our test under some local alternatives: We assume that the parameters $\beta_{2}$ are given by $\beta_{2}=\frac{\tilde{\beta}_{2}}{\sqrt{N}}$ with $\tilde{\beta}_{2} \neq 0$,.i.e. we consider them to be very small, getting smaller when the sample size gets larger.

Theorem 21 Under $H_{1}$, i.e. $\beta_{2} \neq 0$, and the assumptions we state below, we have $z_{N}(\gamma) \Rightarrow z(\gamma)$, where $z(\gamma)$ is a $k_{z}$ dimensional Gaussian process with mean function

$$
\eta(\gamma)=p \lim _{N \rightarrow \infty} \frac{1}{\sqrt{N}} Z_{(\gamma)}^{\prime} M Z_{(\gamma)} \beta_{2}
$$

and variance function

$$
\sigma\left(\gamma_{1}, \gamma_{2}\right)=\lim _{N \rightarrow \infty} \sigma^{2} \frac{1}{N} Z_{\left(\gamma_{1}\right)}^{\prime} M Z_{\left(\gamma_{2}\right)}
$$

Further by the continuous mapping theorem

$$
i z_{N} \rightarrow i z=\int \mathcal{S}\left(z_{N}(\gamma) z_{N}^{\prime}(\gamma)\right) d \gamma \quad \text { in } \quad \text { distribution. }
$$

Proof. We postpone the proof till the end of the appendix.

This result is the first step in deriving the asymptotic distribution of the test statistic. In the derivation we will also need the following two lemmas which are versions of Mercers Theorem. The lemma's are also stated in Bierens and Ploberger (1995).

Lemma 22 (Mercer 's Theorem) Let $\Psi\left(\gamma_{1}, \gamma_{2}\right)$ be a real valued positive semi-definite continuous function on $\Gamma \times \Gamma$, where $\Gamma$ is a compact space, and let $\mu$ be a probability measure on $\Gamma$. The solutions $\lambda_{i}$ and $\psi_{i}(\bullet), i=1,2,3, \ldots$ of the Eigenvalue problem $\int \Psi\left(\gamma_{1}, \gamma_{2}\right) \psi_{i}\left(\gamma_{2}\right) d \mu\left(\gamma_{2}\right)=\lambda_{i} \psi_{i}\left(\gamma_{1}\right)$ are real valued and the function $\Gamma$ has the series representation $\Psi\left(\gamma_{1}, \gamma_{2}\right)=\sum_{i=1}^{\infty} \lambda_{i} \psi_{i}\left(\gamma_{1}\right) \psi_{i}\left(\gamma_{2}\right)$, where the series involved converges uniformly on $\Gamma \times \Gamma$.

Lemma 23 Let the conditions of Lemma 23 be satisfied. The Eigenvalues $\lambda_{i}$ are nonnegative and satisfy $\sum_{i=1}^{\infty} \lambda_{i}<\infty$. Moreover, the Eigenfunctions $\psi_{i}(\bullet)$ are continuous and can be chosen orthonormal and complete in the space $C(\Gamma)$ of continuous real functions on $\Xi$ as well on the space $L_{2}(\mu)$ of squared integrable functions with respect to $\mu$, i.e. $\int \psi_{i}(\gamma) \psi_{j}(\gamma) d \mu(\gamma)=I(i=j)$, and every function $\phi$ in $C(\Gamma)$ or $L_{2}(\mu)$ can be written as

$$
\phi(\gamma)=\sum_{i=1}^{\infty} g_{i} \psi_{i}(\gamma) \quad \text { a.s. } \quad L_{2}(\mu),
$$

with Fourier coefficients

$$
g_{i}=\int \phi(\gamma) \psi_{i}(\gamma) d \mu(\gamma)
$$

satisfying $\sum_{i=1}^{\infty} g_{i}^{2}<\infty$.

We now apply these two lemmas. Let $\Psi$ in Lemma 22 be

$$
\Psi\left(\gamma_{1}, \gamma_{2}\right)=\mathcal{S}\left(\sigma\left(\gamma_{1}, \gamma_{2}\right)\right)
$$


Then the continuity of the Gaussian process $z(\gamma)$, and the compactness of $\Gamma$ imply that $z(\gamma)$ is squared integrable. Further since the set $\left\{\psi_{i}, i=1,2, \ldots\right\}$ of Eigenfunctions is complete we and can apply Parseval 's identity

$$
\begin{aligned}
\int \mathcal{S}\left(z(\gamma) z^{\prime}(\gamma)\right) d \mu(\gamma) & =\int \mathcal{S}(z(\gamma))^{2} d \mu(\gamma) \\
& =\sum_{i=1}^{\infty}\left(\int \mathcal{S}(z(\gamma)) \psi_{i}(\gamma) d \mu(\gamma)\right)^{2} .
\end{aligned}
$$

Observe that $\phi(\gamma)=S(z(\gamma))$. By the fact that the sum of Gaussian processes is again Gaussian it follows that the Fourier coefficients

$$
\int \mathcal{S}(z(\gamma)) \psi_{i}(\gamma) d \mu(\gamma), \quad i=1,2,3 \ldots
$$

are Gaussian too. For the characterization of their joint distribution we only need the covariances and means. The covariances are given by

$$
\begin{aligned}
& E\left[\int \mathcal{S}(z(\gamma)-\eta(\gamma)) \psi_{i}(\gamma) d \mu(\gamma) \int \mathcal{S}(z(\gamma)-\eta(\gamma)) \psi_{j}(\gamma) d \mu(\gamma)\right] \\
= & \iint \mathcal{S}\left(\sigma\left(\gamma_{1}, \gamma_{2}\right)\right) \psi_{i}\left(\gamma_{1}\right) \psi_{j}\left(\gamma_{2}\right) d \mu\left(\gamma_{1}\right) d \mu\left(\gamma_{2}\right)=\lambda_{i} I(i=j),
\end{aligned}
$$

which means that the sequence (5.29) is independent. Further it is easy to see that the mean of the $i$-th element of the sequence (5.29) is just the $i$-th Fourier coefficient of $\eta(\gamma)$

$$
\eta_{i}=\int \eta(\gamma) \psi_{i}(\gamma) d \mu(\gamma)
$$

We note that the line of the above proof is similar to Bierens and Ploberger (1995). From the above it directly follows that under the local alternative $\beta_{2}=\frac{\tilde{\beta}_{2}}{\sqrt{N}}$ with $\tilde{\beta}_{2} \neq 0$ the following theorem holds.

Theorem 24 Under $H_{1}$, i.e. $\beta_{2} \neq 0$, we have

$$
i z=\int \mathcal{S}\left(z_{N}(\gamma) z_{N}^{\prime}\left(\gamma^{\prime}\right)\right) d \gamma \backsim \sum_{j=1}^{\infty}\left(\eta_{j}+\epsilon_{j} \sqrt{\lambda_{j}}\right)^{2}
$$

where $\epsilon_{j}$ is i.i.d $N(0,1)$ and $\lambda_{j}$ and $\eta_{j}$ are as described above.

Under the null we have

$$
H_{0}: \quad T_{0}=\sum_{j=1}^{\infty} \epsilon_{j}^{2} \lambda_{j},
$$

where the $\lambda_{j}$ depend on $\Psi$, and are therefore data dependent. This implies that the asymptotic distribution of $T_{0}$ also depends on the data. However using the theorem below we can obtain critical values which are data independent. 
Theorem 25 (Theorem 5 of Bierens and Ploberger (1995)) Let $\varepsilon_{j}$ be i.i.d N(0,1) and Let

$$
\bar{W}=\sup _{T \geq 1} \frac{1}{N} \sum_{j=1}^{N} \varepsilon_{j}^{2}
$$

For $\eta>0, P\left(T_{H_{0}}>\eta E\left(T_{H_{0}}\right)\right) \leq P(\bar{W}>\eta)$, where $T_{H_{0}}$ is the random variable defined by 5.30. Consequently under the null hypothesis $\beta_{2}=0$

$$
\lim _{N \rightarrow \infty} P\left(i z_{H_{0}}>\eta \int S\left(\sigma_{N}(\gamma, \gamma)\right) d \gamma\right) \leq P(\bar{W}>\eta)
$$

So that our proof of the consistency of our test and its behavior under the giwen local alternative is complete if we can set out conditions under which Theorem 1 holds.

Before we give these conditions we note that one can simulate the distribution of $\bar{W}$. Using 10,000 replications Bierens and Ploberger obtain the following results

$$
P(\bar{W}>3.23)=0.10, \quad P(\bar{W}>4.26)=0.05, \quad P(\bar{W}>6.81)=0.01 .
$$

i.e. this means that reject the null hypothesis at a $10 \%$ significance level if

$$
\int \mathcal{S}\left(z_{N}(\gamma) z_{N}^{\prime}(\gamma)\right) d \gamma / \mathcal{S}\left(\sigma_{N}(\gamma, \gamma)\right) d \gamma>3.23
$$

We now state the proof of Theorem 1. In what follows we use the following definitions:

$$
\begin{array}{ll}
V_{11}=E\left(X_{i}^{\prime} X_{i}\right) & \Omega_{11}=E\left(X_{i}^{\prime} X_{i} \varepsilon_{i}^{2}\right) \\
V_{12}(\gamma)=E\left(X_{i}^{\prime} Z_{i} \mid I\left(q_{i}=\gamma\right)\right) & \Omega_{12}(\gamma)=E\left(X_{i}^{\prime} Z_{i} \varepsilon_{i}^{2} \mid I\left(q_{i}=\gamma\right)\right) \\
V_{21}(\gamma)=E\left(Z_{i}^{\prime} X_{i} \mid I\left(q_{i}=\gamma\right)\right) & \Omega_{21}(\gamma)=E\left(Z_{i}^{\prime} X_{i} \varepsilon_{i}^{2} \mid I\left(q_{i}=\gamma\right)\right) \\
V_{22}(\gamma)=E\left(Z_{i}^{\prime} Z_{i} \mid I\left(q_{i}=\gamma\right)\right) & \Omega_{22}(\gamma)=E\left(Z_{i}^{\prime} Z_{i} \varepsilon_{i}^{2} \mid I\left(q_{i}=\gamma\right)\right)
\end{array}
$$

and

$$
V(\gamma)=\left[\begin{array}{ll}
V_{11} & V_{12}(\gamma) \\
V_{21}(\gamma) & V_{22}(\gamma)
\end{array}\right], \quad \Omega(\gamma)=\left[\begin{array}{ll}
\Omega_{11} & \Omega_{12}(\gamma) \\
\Omega_{21}(\gamma) & \Omega_{22}(\gamma)
\end{array}\right] .
$$

Finally we also need some $s-$ th conditional moments of these variables:

$$
\begin{array}{ll}
V_{11}^{s}=E\left(X_{i}^{\prime} X_{i}\right)^{s}, & \Omega_{11}^{s}=E\left(X_{i}^{\prime} X_{i} \varepsilon_{i}^{2}\right)^{s} \\
V_{12}^{s}(\gamma)=E\left(\left(X_{i}^{\prime} Z_{i}\right)^{s} \mid I\left(q_{i}=\gamma\right)\right), & \Omega_{12}^{s}(\gamma)=E\left(\left(X_{i}^{\prime} Z_{i} \varepsilon_{i}^{2}\right)^{s} \mid I\left(q_{i}=\gamma\right)\right), \\
V_{21}^{s}(\gamma)=E\left(\left(Z_{i}^{\prime} X_{i}\right)^{s} \mid I\left(q_{i}=\gamma\right)\right), & \Omega_{21}^{s}(\gamma)=E\left(\left(Z_{i}^{\prime} X_{i} \varepsilon_{i}^{2}\right)^{s} \mid I\left(q_{i}=\gamma\right)\right), \\
V_{22}^{s}(\gamma)=E\left(\left(Z_{i}^{\prime} Z_{i}\right)^{s} \mid I\left(q_{i}=\gamma\right)\right), & \Omega_{22}^{s}(\gamma)=E\left(\left(Z_{i}^{\prime} Z_{i} \varepsilon_{i}^{2}\right)^{s} \mid I\left(q_{i}=\gamma\right)\right)
\end{array}
$$

and

$$
V^{s}(\gamma)=\left[\begin{array}{ll}
V_{11}^{s} & V_{12}^{s}(\gamma) \\
V_{21}^{s}(\gamma) & V_{22}^{s}(\gamma)
\end{array}\right], \quad \Omega^{s}(\gamma)=\left[\begin{array}{ll}
\Omega_{11}^{s} & \Omega_{12}^{s}(\gamma) \\
\Omega_{21}^{s}(\gamma) & \Omega_{22}^{s}(\gamma)
\end{array}\right] .
$$

We further introduce $f(q)$ as the probability density of the variable $q$. Assumption The assumptions we make here are in essence identical to Hansen (1996) and (1997). 
1. Let the series $\left(X_{i}, q_{i}, \varepsilon_{i}\right)$ be strictly stationary with $\beta$ mixing coefficients $\beta_{m}$ satisfying

$$
\beta_{m}^{(s-1) / 2 s}=O\left(m^{-(1+\delta)}\right)
$$

2. $E\left(\varepsilon_{i} \mid F_{i-1}\right)=0$;

3. $E\left|X_{i}\right|^{2 \cdot s}<\infty$, and $E\left|\varepsilon_{i}\right|^{2 s}<\infty$;

4. $f(\gamma), V(\gamma), \Omega(\gamma), V^{s}(\gamma), \Omega^{s}(\gamma)$ be continuous at $\gamma=\gamma_{0}$;

5. $V(\gamma), \Omega(\gamma)$, are positive definite and $f(\gamma)>0$;

6. $P\left(q_{i} \in \Gamma\right)<1$.

A discussion of these assumptions is given in Hansen (1996). Under these assumptions we have the following results:

Lemma $26 \frac{1}{\sqrt{N}} Z_{(\gamma)}^{\prime} \varepsilon \Longrightarrow G(\gamma)$, a zero mean Gaussian process with covariance kernel $\sigma^{2} V_{22}(\gamma)$ where $V_{22}(\gamma)$ is defined as above with $\gamma=\min \left(\gamma_{1}, \gamma_{2}\right)$.

The proof follows directly from Theorem 3 of Hansen (1997) and the above definitions.

We are now ready to prove Theorem 21 .

Proof of theorem 21 Define $X_{(\gamma)}^{*}=\left[X Z_{(\gamma)}\right]$ then we have for fixed $\gamma_{0}$

$$
\frac{1}{\sqrt{N}} X_{\left(\gamma_{0}\right)}^{* \prime} \varepsilon=\left[\begin{array}{l}
\frac{1}{\sqrt{N}} X^{\prime} \varepsilon \\
\frac{1}{\sqrt{N}} Z_{\left(\gamma_{0}\right)}^{\prime \prime} \varepsilon
\end{array}\right] \rightarrow N\left(\left(\begin{array}{l}
0 \\
0
\end{array}\right), \sigma^{2}\left[\begin{array}{ll}
V & V_{12}\left(\gamma_{0}\right) \\
V_{21}\left(\gamma_{0}\right) & V_{22}\left(\gamma_{0}\right)
\end{array}\right]\right) .
$$

We have also weak convergence

$$
\frac{1}{\sqrt{N}} X_{(\gamma)}^{* \prime} \varepsilon=\left[\begin{array}{l}
\frac{1}{\sqrt{N}} X^{\prime} \varepsilon \\
\frac{1}{\sqrt{N}} Z_{(\gamma)}^{x} \varepsilon
\end{array}\right] \Longrightarrow\left[\begin{array}{l}
N\left(0, \sigma^{2} V\right) \\
G(\gamma)
\end{array}\right]
$$

where $G(\gamma)$ is defined as in Lemma 26. Now we observe that we have

$$
\begin{aligned}
\frac{1}{\sqrt{N}} Z_{(\gamma)}^{\prime} M \varepsilon & =\frac{1}{\sqrt{N}} Z_{(\gamma)}^{\prime}\left(I-X\left(X^{\prime} X\right)^{-1} X^{\prime}\right) \varepsilon \\
& =\frac{1}{\sqrt{N}} Z_{(\gamma)}^{\prime} \varepsilon-\left(\frac{1}{N} Z_{(\gamma)}^{\prime} X\right)\left(\frac{1}{N} X^{\prime} X\right)^{-1}\left(\frac{1}{\sqrt{N}} X^{\prime} \varepsilon\right) \\
& =\left[-\left(\frac{1}{N} Z_{(\gamma)}^{\prime} X\right)\left(\frac{1}{N} X^{\prime} X\right)^{-1} I\right] \frac{1}{\sqrt{N}} X_{(\gamma)}^{*} \varepsilon \\
& \Longrightarrow G^{*}(\gamma)
\end{aligned}
$$

where $G^{*}(\gamma)$ is a zero mean Gaussian process with covariance kermel

$$
\begin{aligned}
& -\left(\left(V_{21}\right)\left(V_{11}\right)^{-1}\right)\left(\sigma^{2} V_{11}\right)\left(\left(V_{21}\right)\left(V_{11}\right)^{-1}\right)^{\prime}+\sigma^{2} V_{22}(\gamma) \\
= & \sigma^{2}\left(-Z_{(\gamma)}^{\prime} X\left(X^{\prime} X\right)^{-1} X^{\prime} Z_{(\gamma)}+Z_{(\gamma)}^{\prime} Z_{(\gamma)}\right) \\
= & \sigma^{2} Z_{(\gamma)}^{\prime} M Z_{(\gamma)} .
\end{aligned}
$$

The theorem follows. 


\section{Chapter 6}

\section{Conclusions}

\subsection{Introduction}

In this thesis we studied the possibilities of jointly analyzing dynamic models for a fixed set of individual entities. To improve efficiency and to allow identification of common and individual parameters we explored restrictions across individuals. It is shown that imposing cross-individual restrictions indeed leads to more efficiency in parameter estimation and consequently to additional power of statistical tests. We point out that we can only introduce and identify common and individual features by jointly modelling the data for all individuals of interest. Thereby one can impose cross-individual restrictions which reffect similarities and common features among individuals or groups of individuals.

The resulting models can be used for a variety of problems where it is likely that individual entities have some similar features. In macroeconomic applications the entities usually represent countries or regions. Three macroeconomic applications at a country level were carried out and are presented in this thesis. It is shown that imposing crosscountry restrictions indeed leads to a more powerful statistical analysis compared to an analysis based on the individual countries data alone. The analysis of dynamic models for several individuals jointly with common as well as specific parameters creates some interesting econometric problems. In this thesis we investigated and solved several of these problems.

\subsection{Summary of the results}

We started out by investigating the estimation of $\operatorname{AR}(\mathrm{p})$ models when dynamic panel data are available using Maximum Likelihood. We restrict the lag coefficients for each individual unit to be the same while we leave the intercept to vary freely over the individual units. For the ML estimator in an AR(p) model in levels we derived the asymptotic bias in case the number of individuals is infinite and the number of observed time periods finite. Under the same assumption we derived the asymptotic distribution of the consistent ML estimator of first differenced data. Using a simulation we investigated the small sample performance of these two ML estimators.

We find that ML based on the first differenced data outperforms uniformly ML based 
on the non-transformed data. We further find in a simulation that in small samples the actual variances approximate the asymptotic variances well. The asymptotic approximation of the bias of the ML estimator in the non-transformed model also shows up in the small samples.

In chapter three we investigated whether pooling, restricting the parameters for all individuals to be the same, improves one step ahead forecasting. First we show that the second weak order mean square error criterium gives a good indication of whether pooling will improve forecasting. Second, we show that identifying contemporaneous correlations between individuals also improves forecasting, using a mean squared forecast error criterion. These findings are confirmed by a small sample size simulation with a leading indicator model for 6 individuals with an imposed block diagonal contemporaneous covariance matrix.

An empirical application of postwar international real GDP growth rates for 18 OECD countries illustrates these findings. When allowing for contemporaneous residual correlation across countries, strict pooling restrictions have to be rejected but the second weak criterion indicates that pooling could improve forecasting. And indeed GLS-based pooled forecast are found to outperform GLS-based individual and OLS-based pooled and individual forecasts. We note however that OLS outperforms GLS when comparing the root mean square forecasting errors of one step ahead forecasts.

In the second part of the thesis we consider the issue of pooling sets of multivariate models. In the chapters four and five we introduced two new Vector Autoregressive models, with the purpose to investigate the specific features of panel data sets.

In chapter four we introduced vector autoregressive models for a fixed number of individuals where the individual means are left unrestricted but the lag coefficients are restricted to be the same across individuals. We further consider some decompositions of the contemporaneous covariance matrix into products of matrices of either individual specific parameters or parameters common to all individuals.

We derive the asymptotic distribution of the ML estimators and use these to derive asymptotic confidence bounds for orthogonalized and nonorthogonalized impulse response functions. It is shown that imposing the restrictions leads to tighter confidence bounds. Using the same data set as in chapter three we also find empirically tighter confidence bounds. We identified and estimated trivariate models for real GDP-growth, real money and inflation for Austria, Belgium, Germany, The Netherlands, The United Kingdom and Switzerland jointly. The narrower confidence bounds for the analysis based on the joint model with imposed restrictions leads indeed to statistically more significant results and allows for a better economic interpretation. This type of models appears to be a possibly important tool for economic policy analysis.

In chapter five we introduced linear and threshold VAR models where we assumed all parameters to be the same across entities, except allowing for heteroscedasticity. We allow for regime shifts over time. We developed a new test for the existence of an endogenously determined threshold. The test has the advantage above other tests, such as the average, exponential and sup LM tests, that it does not depend on the choice of some nuisance parameters or the dimension of the system. This advantage is obtained at the price of possibly losing some statistical power.

As an illustration we used a country-level panel data set. We investigated whether 
a country's savings rate matters for the relationship between GDP-growth and openness to trade. We tested, using several tests, for the existence of an endogenous threshold separating high from low savings regimes. Once the existence of a threshold was established, estimation of the threshold VAR models revealed that for countries in the high savings regime, lagged openness has a positive effect on growth, while the opposite holds for countries in the low savings regime. Furthermore, there are striking differences in the impulse response analysis between the two regimes. In the high savings regime, a positive shock in openness leads to higher GDP growth in all subsequent years, while in the low savings regime, the initially positive GDP growth rate becomes negative after a few years.

\subsection{Final conclusions}

The analysis of dynamic models for several individuals jointly will become more important as more data become available. Modelling individual units jointly creates difficulties but opens up new possibilities. First one can identify individual and common parameters and investigate similarities or differences among individuals. Second, although we did not consider this possibility in this thesis, one can identify time effects to capture features which evolve over time. Thirdly one can impose cross-individual restrictions to model more efficiently the information in the data and in this way gaining statistical power.

To fully exploit all of the above possibilities we need estimators which have good small sample size properties. This problem is already evident in situations where $T$ is fixed and large $N$ asymptotics have to be used. The finite sample problem however is difficult to solve in general. The construction of good estimators and tests along with their statistical properties will determine the pace at which we can deal with economically relevant questions based on data sets available. It also determines which problems we can handle and which we cannot.

This is illustrated by the choices we made in the chapters four and five. In chapter four we only considered a fixed and small number of individuals observed for a large number of time periods to let the large $T$ asymptotics be justified. In chapter five we used a pooled model because we were not able to prove the correctness of the tests for the existence of a threshold in a model with fixed effects. As we have data for many countries we could not justify the assumption of a small fixed number of countries.

This brings us to the suggestion that further research should be directed towards developing estimation and inference techniques for different assumptions on the number of individuals and observed time periods, preferably within a finite sample size context. 


\section{Bibliography}

Ahn, S.C., Schmidt, P. (1995). "Efficient estimation of models for dynamic panel data ", Journal of Econometrics 68, 5-27.

Ahn, S.C., Schmidt, P. (1997). "Efficient estimation of dynamic panel data models: Alternative assumptions and simplified estimation", Journal of Econometrics 76, 309-321.

Akaike, H. (1973). "Information theory and an extention of the maximum likelihood principle", in B.N. Petrov \& F. Csáki (eds.). 2nd International Conference on Information Theory, Budapest: Académiai Kiadó, 267-281.

Akaike, H. (1974). "A new look at the statistical model identification", IEEE Transactions on Automatic Control, AC-19, 716-723.

Amemiya, T. (1985). Advanced Econometrics, Basil Blackwell, Oxford.

Amemiya, T., MaCurdy, T.E. (1986). "Instrumental-variable estimation of an errorcomponents model ", Econometrica 54, 869-881.

Anderson, T.W., Hsiao, C. (1982). "Formulation and estimation of dynamic models using panel data", Journal of Econometrics 18, 47-82.

Andrews, D.W.K, Lee, I, Ploberger, W. (1996). "Optimal changepoint tests for normal linear regression", Journal of Econometrics 70, 9-38.

Andrews, D.W.K., Ploberger W. (1994). "Optimal tests when a nuisance parameter is present only under the alternative", Econometrica 62, 1383-1414.

Andrews, D.W.K. (1993). "Tests for parameter instability and structural change with unknown change point", Econometrica $61,821-856$.

Arellano, M., Bond, S. (1991). "Some tests of specification for panel data: Monte Carlo evidence and an application to employment equations", Review of Economic Studies 58, 277-297.

Arellano, M., Bover, O. (1995). "Another look at the instrumental variable estimation of error-components models", Journal of Econometrics 68, 29-51.

Bai, J. (1995). "Estimation of structural change based on Wald-type statistics", working paper, Department of Economics, M.I.T.

Baillie, R.T. (1987). "Inference in dynamic models containing 'surprise" variables", Journal of Econometrics 35, 101-117.

Balassa, B. (1985). "Exports, policy choices, and economic growth in developing countries after the 1973 oil price shock", Journal of Developing Economics 18(2), 23-35.

Balassa, B. (1989). "Outward orientation," in Handbook of development economics, edited by H. Chenery and T.N. Srinivasan, Amsterdam: North-Holland, 1645-89. 
Balke, N.S., Chang C. (1995). "Credit and economic activity: shocks or propagation mechanism?", working paper Southern Methodist University.

Baltagi, B.H. (1995). Econometric Analysis of Panel Data, Chichester, John Wiley and Sons.

Baltagi, B.H., Griffin, J.M. (1983). "Gasoline demand in the OECD: An application of pooling and testing procedures", European Economic Review 22, 117-137.

Baltagi, B.H., Griffin, J.M. (1983). "Pooled estimators vs. their heterogeneous counterparts in the context of dynamic demand for gasoline", Journal of Econometrics 77, 303-327.

Bernard, A.B., Durlauf, S.N. (1995). "Convergence in international output", Journal of Applied Econometrics 10, 97-108.

Bierens, H.J., Ploberger, W. (1997). "Asymptotic theory of integrated conditonal moment tests", Econometrica, Forthcoming.

Bilson, J.F.O. (1981). "The 'speculative efficiency' hypothesis", Journal of Business 54, 435-451.

Blanchard, O.J., Quah, D. (1989). "The dynamic effects of aggregate demand en supply disturbances", American Economic Review, 655-673.

Blattberg, R.C., George, E.I. (1991). "Shrinkage estimation of promotional elasticities: Seemingly unrelated equations", Journal of the American Statistical Association 86, 304315.

Bosworth, B. (1993). "Saving and investment in a global economy", Washington: Brookings Institution.

Breuch, T.S., Mizon, G.E., Schmidt, P. (1989). "Efficient estimation using panel data", Econometrica 57, 695-700.

Breusch, T.S., Pagan, A.R. (1980). "The Lagrange multiplier test and its applications to model specifications in econometrics", Review of Economic Studies 47, 239-253.

Carroll, C.D., Weil, D.N. (1993). "Saving and growth: a reinterpretation", NBER Working Paper No. 4470.

Chamberlain, G. (1980). "Analysis of covariance with qualitative data", Review of Economic Studies 47, 225-238.

Chan, K.S. (1993). "Consistency and limiting distribution of the least squares estimator of a threshold autoregressive model", Annals of Statistics 21, 4520-533.

Chib, S., Greenberg, E. (1995). "Hierarchical analysis of SUR models with extensions to correlated serial errors and time-varying parameter models", Journal of Econometrics 68, 339-360.

Chow, G.C. (1960). "Tests of equality between sets of coefficients in two linear regressions", Econometrica 28, 591- 605 . 
Davidson, R., MacKinnon, J.G. (1993). Estimation and inference in econometrics, Oxford University Press, New York.

Edwards, S. (1992). "Trade orientation, distortions, and growth in developing countries", Journal of Development Economics 39, 31-57.

Edwards, S. (1993). "Openness, trade liberalization, and growth in developing countries", Journal of Economic Literature 31, 1358-1393.

Esfahani, H.S. (1991). "Exports, imports, and economic growth in semi-industrialized countries", Journal of Development Economics 35(1). 93-116.

Feenstra, R. (1996). "Trade and uneven growth", Journal of Development Economics 49(1). 199-227.

Gallant, A., Rossi, P.E., Tauchen, G. (1993). "Nonlinear dynamic structures", Econometrica 61(4). 871-908.

Galbraith, R.F., Galbraith, J.I. (1974). "On the inverses of some patterned matrices arising in the theory of stationary time series", Journal of Applied Probability 11,63-71.

Garcia-Ferrer, A., R.A. Highfield, F. Palm, Zellner, A. (1987). "Macroeconomic forecasting using pooled international data", Journal of Business and Economic Statistics 5, 53-67.

Giannini, C. (1992). Topics in structural VAR Econometrics, New York, Springer Verlag. Goodnight, J., Wallace, T.D. (1972). "Operational techniques and tables for making weak MSE tests for restrictions in regressions", Econometrica 40, 699-709.

Gordon, R.H., Bovenberg, A.L. (1996). "Why is capital so immobile internationally? Possible explanations and implications for capital Income taxation", American Economic Review 86(5). 1057-1075.

Gupta, K.L., Islam, M.A. (1983). "Foreign capital, savings, and growth", International Studies in Economics and Econometrics, Vol. 9, D. Reidel Publishing Company, Dordrecht, Holland.

Hamilton, J.D. (1994). Time Series Analysis, Princeton, Princeton University Press.

Hannan, E.J., Quinn, B.G. (1979). "The determination of the order of an autoregression", Journal of the Royal Statistical Society, B41, 190-195.

Hansen, B.E. (1995). "Endogenous sample splitting and the estimation of thresholds", Boston College, manuscript.

Hansen, B.E. (1996). "Inference when a nuisance parameter is not identified under the null hypothesis", Econometrica 64, 413-430.

Hansen, B.E. (1996a). "Sample splitting and threshold estimation", Boston College, Manuscript.

Harrison, A. (1996). "Openness and growth: A time-series, cross-country analysis for 
developing countries", Journal of Developing Economics 48, 419-447.

Hsiao, C. (1986). Analysis of Panel Data, Cambridge, University Press.

Holtz-Eakin, D., Newey, W., Rosen, H. (1988). "Estimating vector autoregressions with panel data", Econometrica 56, 1371-1395.

Im, K., Pesaran, M. H., Shin, Y. (1996). "Testing for unit roots in heterogeneous panels", manuscript, University of Cambridge.

Islam, N. (1995). "Growth empirics: A panel data approach", The Quarterly Journal of Economics 110, 1127-1170.

Jorgenson, D.W., Ho, M.S. (1993). "Trade policy and U.S. economic growth", HIER Discussion Paper No. 1634.

Judson, A.J., Owen, A.L. (1997). "Estimating dynamic panel data models: A practical guide for macroeconomists", Working paper 1997-3, Finance and Economics Discussion Series, Federal Reserve Board, Washington, D.C.

Kievit, J.F. (1995). "On bias, inconsistency and efficiency of various estimators in dynamic panel data models ", Journal of Econometrics 68, 53-78.

Lancaster, T., Lindenhovius, B. (1996) "Biases in dynamic panel data models: A reconsideration" (First Revision). Working paper no. 96-11 Brown University.

Lee, J-L. (1995). "Capital goods imports and long-run growth", Journal of Development Economics 48, 91-110.

Levine, R., Renelt, D. (1992). "A sensitivity analysis of cross-country growth regressions", American Economic Review 82(4). 942-963.

Litkepohl, H. (1989). "A note on the asymptotic distribution of impulse response functions of estimated VAR models with orthogonal residuals", Journal of Econometrics 42, 371-376.

Luttkeponl, H. (1990). "Asymptotic distributions of impulse response functions and forecast error decompositions of vector autoregressive models", Review of Economics and Statistics 72, 116-125.

Lútkepohl, H., Reimers H.E. (1992). "Impulse response analysis of cointegrated systems", Journal of Economic Dymamics and Control 16, 53-78.

Liutkepohl, H. (1991). Introduction to Multiple Time Series Analysis, Springer Verlag, Berlin-Heidelberg.

Lütkepohl, H. (1996). Handbook of Matrices, Chichester, John Wiley and Sons.

Maddala, G.S. (1991). "To pool or not to pool: That is the question", Journal of Quantitative Economics 7, 255-264.

Maddala, G.S., Trost, R.P., Li, H. (1994). "Estimation of short run and long run elasticitjes of energy demand from panel data using shrinkage estimators", paper presented at 
the ESEM, Maastricht.

Maddison, A. (1992). "A long run perspective on saving", in Saving behavior: theory international evidence, and policy implications, edited by E. Koskela and J. Paunio, Cambridge: Blackwell, 27-59.

Magnus, J.R. (1978). "Maximum likelihood estimation of the GLS model with unknown parameters in the disturbance covariance matrix", Journal of Econometrics 7, 281-312.

Magnus, J.R. (1988). Linear Structures (Charles Griffin \& Company Ltd., London).

Magnus, J.R., Neudecker, H.(1986). "Symmetry, 0-1 matrices and Jacobians: A Review", Econometric Theory 2, 157-190.

Magnus, J.R., Neudecker, H. (1991). "Matrix differential calculus with applications in statistics and econometrics", Wiley, Chichester.

Mankiw, N.G. (1986). "The term structure of interest rates revisited (with discussions by S.M. Goldfeld and R.J. Shiller)", Brookings Papers on Economic Activity 1, 61-109.

Mankiw, N.G, Romer, D., Weil, D.N. (1992). "A contribution to the empirics of economic growth", "The Quarterly Journal of Economics 108, 407-437.

Mátyas, L., Sevestre, P. (eds.) (1996). The Econometrics of Panel Data: A H.andbook of the Theory with Applications, 2nd ed., Dordrecht, Kluwer Academic Publishers.

McElroy, M.B. (1977). "Weaker MSE criteria and tests for linear restrictions in regression models with non-spherical disturbances", Journal of Econometrics 6, 389-394.

Min, C., Zellner, A. (1993). "Bayesian and non-Bayesian methods for combining models and forecasts with applications to forecasting international growth rates", Journal of Econometrics 56, 89-118.

Mittnik, S. (1990). "Macroeconomic forecasting using pooled international data", Journal of Business and Economic Statistics 8, 205-208.

Mittnik, S., Zadrosky, P.A. (1993). "Asymptotic distributions of impulse responses, step responses, and variance decompositions of estimated linear dynamic models", Econometrica $61,857-870$.

Mundlak, Y. (1978). "On the pooling of time series and cross section data", Econometrica $46,69-85$.

Nehru, V., Dhareshwar, A. (1993). "A new database on physical capital stock: Sources, methodology, and results, Revista de Analisis Economico 8(1). 37-59.

Nerlove, M. (1996). "Growth rate convergence, fact or artifact?", Working Paper, Department of Agricultural and Resource Economics, University of Maryland.

Nickell, S. (1981). "Biases in dynamic models with fixed effects", Econometrica 48, 14161426.

Osang, T., Pereira, A. (1996a). "Savings, volume of trade, and growth", forthcoming in 
Review of International Economics.

Osang, T., Pereira, A. (1996b). "Foreign growth and domestic performance in a small open economy", forthcoming in Journal of International Economics.

Palm, F.C., Sneek, J.M. (1984). "Significance tests and spurious correlation in regression models with autocorrelated errors", Statistical Papers, 25, 87-105.

Picard, D. (1985). "Testing and estimating change-points in time series model ", Stochastic Processes and Their Applications 19, 297-303.

Potter, S. (1995). "A non-linear approach to US GNP ", Journal of Applied Econometrics $10,109-125$.

Quah, D., Rauch, J.E. (1990). "Openness and the rate of economic growth", Prelimanary draft, Ec. dept., MTT, Cambridge, and UCSD, San Diego.

Quinn, B.G. (1980). "Order determination for a multivariate autoregression", Journal of the Royal Statistical Society, B42, 182-185.

Ridder, G., Wansbeek, T. (1990). "Dynamic models for panel data", in F. van der Ploeg (ed)., Advanced Lectures in quantitative Economics, Academic Press, London, 557-582.

Rivera-Batiz, L., Romer, P. (1991). "Economic integration and endogenous growth", Quarterly Journal of Economics 106, 531-555.

Schwarz, G (1978). "Estimating the dimension of a model", Annals of Statistics 6, 461464.

Sevestre, P., Trognon, A. (1985). "A note on autoregressive error components models", Journal of econometrics $28,231-245$.

Sims, C.A. (1980). "Macroeconomics and reality", Econometrica 48, 1-48.

Stock, J.H., Watson, M.W. (1989). "New indexes of coincident and leading economic indicators" in Blanchard, O.J. and S. Fischer (eds.). NBER Macroeconomics Annual 1989, Cambridge, MA, MIT Press, 351-394.

Thomas, V., N. Halevi, Stanton, J. (1991). "Does policy reform improve performance," Background paper for World Development Report 1991.

Trognon, A. (1978). "Miscellaneous asymptotic properties of ordinary least squares and maximum likelihood estimators in dynamic error components models", Annals de l'INSEE $30-31,632-657$.

Turnovsky, S.J. (1996). "Fiscal policy, growth, and macroeconomic performance in a small open economy", Journal of International Economics 40, 41-66.

Wallace, T.D., Toro-Vizcarrondo, C.E. (1969). "Tables for the mean square error test for exact linear restrictions in regression", Journal of the American Statistical Association 64, 1649-1663.

Wallace, T.D. (1972). "Weaker criteria and tests for linear restrictions in regressions", 
Econometrica 40, 689-709.

Wansbeek, T., Bekker, P. (1996). "On IV, GMM, and ML in a dynamic panel data model", Economics Letter 51, 145-152.

White, H. (1980). "A heteroscedasticity-consistent covariance matrix estimator and a direct test for heteroscedasticity", Econometrica 48, 817-838.

World Data 1995 (1996). World Bank indicators on CD-Rom, The World Bank, Washington, DC.

Zellner, A. (1962). "An efficient method of estimating seemingly unrelated regression and tests for aggregation bias", Journal of the American Statistical Association 57, 348-368.

Zellner, A., Hong, C. (1989). "Forecasting international growth rates using Bayesian shrinkage and other procedures", Journal of Econometrics 40, 183-202.

Zellner, A., Hong, C., Min, C. (1991). "Forecasting turning points in international output growth rates using Bayesian exponentially weighted autoregression, time-varying parameter, and pooling techniques", Journal of Econometrics 49, 275-304.

Zellner, A. (1994). "Time series analysis, forecasting and econometric modelling : the Structural Econometric Modelling, Time Series Analysis (SEMTSA) Approach", Journal of Forecasting 13, 215-233. 


\section{Nederlandse samenvatting}

Bij de empirische analyse van macro-economische vraagstukken wordt vaak gebruik gemaakt van "panel data sets". Dit zijn verzamelingen van data voor meerdere eenheden, bijvoorbeeld landen, met voor elke eenheid waarnemingen gedurende meerdere perioden, bijvoorbeeld jaren. Het statistisch efficiènt analyseren van dergelijke data sets is in de praktijk niet eenvoudig.

Ten eerste heeft de onderzoeker het probleem dat het modelleren van de in de data aanwezige informatie lastig is. Vaak is het moeilijk om relevante individuele en gemeenschappelijke karakteristieken van elkaar te onderscheiden. Hierbij speelt mee dat een panel data set de mogelijkheid biedt om ook veranderingen door de tijd heen en interacties tussen individuen te analyseren. Er zijn een haast onbegrensd aantal mogelijkheden om de gemeenschappelijke en individuele karakteristieken, tijdseffecten en interacties tussen individuen te modelleren. Hierdoor is het geen eenvoudige taak om voor een gegeven probleem het juiste model te vinden.

Ten tweede is het toetsen en schatten van het door de onderzoeker gekozen model vaak lastiger dan in de klassieke tijdreeks of statische regressie analyse. Een voorbeeld hiervan is dat als er gekozen is voor het modelleren van gezamenlijke en individuele karakteristieken, het asymptotisch gedrag van vaak gebruikte schatters af blijkt te hangen van de verhouding tussen het aantal individuen en het aantal geobserveerde perioden. In de praktijk is deze verhouding vaak dusdanig dat het niet duidelijk is welk asymptotisch gedrag de werkelijkheid het beste benadert. Stel, de onderzoeker schat een model op basis van een data set bestaande uit de jaarcijfers voor het bruto nationaal product voor 18 OECD landen gedurende de laatste 25 jaar. Wordt het kleine steekproef gedrag van de schatter het best beschreven door de theorie die er vamuit gaat dat het aantal landen naar oneindig gaat of wordt het gedrag van de schatter beter beschreven door de theorie die als uitgangspunt heeft dat het aantal geobserveerde jaren naar oneindig gaat? We merken op dat er binnen de klasse van modellen die in het kader van dit proefschrift bestudeerd worden, namelijk dynamische panel data modellen, nauwelijks schatters bekend zijn waarvan resultaten bekend zijn die gebaseerd zijn op zowel een eindig aantal individuen als tijdwaarnemingen. In dit proefschrift bestuderen we aspecten van beide bovenstaande problemen binnen de context van dynamische panel data modellen.

In hoofdstuk twee beschouwen we het gedrag van de meest aannemelijke schatter in autoregressieve dynamische panel data modellen. Dit zijn autoregressieve modellen; de waarde van een variabele vandaag wordt verklaard uit het verleden van dezelifde variabele, waarbij alle parameters voor elk individu het zelfde verondersteld worden behalve de intercept. We beschouwen deze modellen zowel onder de veronderstelling dat het aantal individuen oneindig is en het aantal geobserveerde perioden eindig, als het tegenovergestelde geval. De motivatie voor dit hoofdstuk komt voort uit het feit dat de meest aannemelijke schatter in een autoregressief panel data model, geëvalueerd onder de aanname van een oneindig aantal individuen en een eindig aantal geobserveerde perioden, asymptotisch onzuiver is indien we de data in niveaus gebruiken. Dit wordt veroorzaakt door een combinatie van een eindig aantal geobserveerde perioden en een oneindig aantal 
incidentele parameters.

De meest aannemelijke schatter van dezelfde parameters, maar nu in het model met de data in eerste verschillen, is asymptotisch wel zuiver voor een eindig aantal geobserveerde perioden. Voor deze schatter leiden we de asymptotische verdeling expliciet af en geven we een eerste orde benadering voor de asymptotische onzuiverheid van de meest aannemelijke schatter voor de data in niveaus. Dit biedt de mogelijkheid de meest aannemelijke schatter te corrigeren voor deze onzuiverheid.

Verder vergelijken we de asymptotische gedragingen van beide meest aannemelijke schatters in een simulatie met de in de praktijk voorkomende situatie van zowel een eindig aantal individuen als een eindig aantal geobserveerde perioden.

In hoofdstuk drie bestuderen we of pooling, dat wil zeggen het opleggen van de restrictie dat de parameters voor elk individu gelijk zijn, meer precieze voorspellingen genereert. Uit voorgaande onderzoeken is gebleken dat met pooling over het algemeen goede resultaten worden behaald, ook al wordt de formele test op strikte gelijkheid van alle parameters verworpen. Voor een verbetering van de gemiddelde kwadratische voorspelfout is een strikte gelijkheid van de parameters namelijk niet noodzakelijk. Daarom hebben we bestudeerd of toetsen op minder stricte criteria indicaties kunnen geven of pooling al dan niet tot een verbetering in voorspellingen zal leiden. Uit onze analyse blijkt dat dit inderdaad het geval is.

Hierbij is tevens bestudeerd of het analyseren van de covariantie structuur tussen individuen tot verbetering van de voorspelkwaliteit leidt. Dit blijkt het geval te zijn voor de individuele modeilen. Binnen de gepoolde modellen is de verbetering niet duidelijk: Modellen waarbinnen we de covariantie structuur sterker restricteren dan formele toetsen aangeven presteren niet slechter.

Het onderscheidend vermogen van de toetsen en de kwaliteit van de voorspellingen in de praktijk is bestudeerd aan de hand van een simulatie en een data set bestaande uit macro-economische data voor 18 OECD landen. De basis hierbij is een autoregressief leading-indicator model voor het voorspellen van de groei in output in de 18 landen.

In hoofdstuk vier bestuderen we vector autoregressieve modellen voor meerdere individuen gezamenlijk. We bestuderen verschillende mogelijke verzamelingen van restricties. Hierbij staat centraal de restrictie dat voor ieder individu de autoregressieve coëfficiënten gelijk zijn. Verder laten we de intercept wel vrij maar introduceren we vier verschillende mogelijke collecties van restricties op de contemporaine covariantie matrix. De restricties verschillen van elkaar door andere combinaties van individuele en gezamenlijke parameters We analyseren deze modellen met behulp van meest aannemelijke schatter.

De motivatie voor het bestuderen van dergelijke modellen komt voort uit het feit dat voor een vector autoregressief model het aantal parameters explosief toe neemt bij het vergroten van het aantal endogene variabelen. Hierdoor zijn er vaak niet genoeg data beschikbaar om een wolledige analyse te doen op basis van de data voor een individueel land alleen. Tevens is het op een dergelijke manier mogelijk de efficiëntie te vergroten.

Een illustratie hiervan is de impuls respons analyse die we hebben uitgevoerd voor zes Europese landen. Eerst is op basis van een aantal aannemelijkheidsratiotests een model gekozen waarvan de restricties niet door de data verworpen werden. Daarna zijn de impuls respons functies berekend inclusief $95 \%$ betrouwbaarheidsgrenzen. De betrouwbaarheidsintervallen gebaseerd op het model met de geaccepteerde restricties, gebruik makend 
wan de data voor alle landen, blijken inderdaad minder wijd te zijn dan deze gebaseerd op een analyse op basis van data voor de landen individueel. Op basis hiervan kan nu gesteld worden dat voor een bepaald aantal landen bepaalde schokken significant andere variabelen beïnvloeden, terwijl deze conclusie niet kan worden getrokken op basis van de data voor het bepaalde land alleen.

In hoofdstuk vijf bestuderen we de relatie tussen een land's spaarquote, handelsvolume en economische groei. Dit wordt gedaan aan de hand van vector autoregressieve modellen waarin we de mogelijkheid openhouden dat de autoregressieve relatie verandert als de spaarquote een drempelwaarde overschrijdt. Hierbij maken we gebruik van een data set voor 58 landen over de periode 1960-1987, waarbij de data worden gepooled.

We ontwikkelen een procedure voor het toetsen op het bestaan van een drempelwaarde. We bestuderen de eigenschappen van de door ons ontwikkelde procedure en vergelijken deze met andere bekende procedures. We bestuderen de bruikbaarheid van de door ons ontwikkelde procedure in de praktijk door het toetsen van de hypothese of de spaarquote van een land de relatie tussen openheid (handelsvolume) en economische groei beinvloedt. Hierbij is de te toetsen hypothese dat vanaf een zekere drempelwaarde van de spaarquote een vergroting van openheid gepaard gaat met economische groei. In situaties waarin zich op het tijdstip van de vergroting van de openheid de spaarquote zich onder de drempelwaarde bevindt verwachten we geen of zelfs een negatieve economische groei ten gevolge van een verder openstellen van een land's markt. Op basis van de data kan de hypothese van het ontbreken van een drempelwaarde wordt verworpen ten gunste van het bestaan van een drempelwaarde.

In een volgende stap wordt de drempelwaarde geïdentificeerd, en wordt het tweede gedeelte van de hypothese getoetst met behulp van impuls respons analyse. Deze analyse bevestigt inderdaad het bovenbeschreven vermoeden. De analyse wordt verder geillustreerd met de respons functies voor Japan, een land met een hoge spaarquote en de USA, een land met een lage spaarquote. 


\section{Curriculum vitae}

Adriaan Jan Hoogstrate werd geboren op 4 augustus 1968 te Kapelle. In 1987 haalde hij zijn VWO diploma an het Goese Lyceum te Goes. Daarna studeerde hij wiskunde en toegepaste statistiek aan de Universiteit van Amsterdam. Op 23 augustus 1993 studeerde hij af in de Wiskunde en cum laude in de Statistiek. Op 1 september 1993 trad hij in dienst van het NWO als onderzoeker in opleiding en startte hij zijn promotie onderzoek bij de Vakgroep Kwantitatieve Economie aan de Universiteit Maastricht. Van 1 maart tot 1 juni 1996 was hij Visiting Researcher aan de Southern Methodist University. Sinds 1 december 1997 werkt hij als Universitair Docent Econometrie/Statistiek aan de Katholieke Universiteit Brabant. 